\title{
Continuous Flow Preparation of (Hetero)Benzylic Lithiums via Iodine-Lithium Exchange Reaction under Barbier Conditions
}

\section{Supporting Information}

\author{
Niels Weidmannћ, Johannes H. Harenberg $\ddagger$ Paul Knochel* \\ Department of Chemistry, Ludwig-Maximilians-Universität Munich, \\ Butenandtstr. 5 - 13, 81377 Munich, Germany \\ E-Mail:knochel@cup.uni-muenchen.de
}

Table of Contents

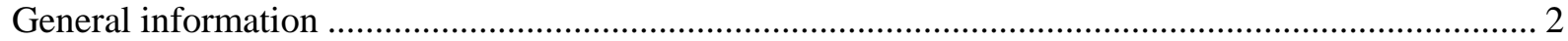

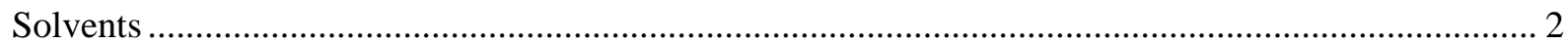

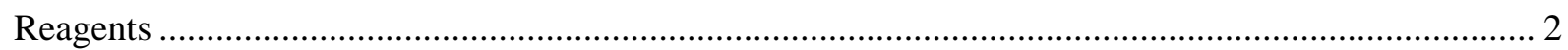

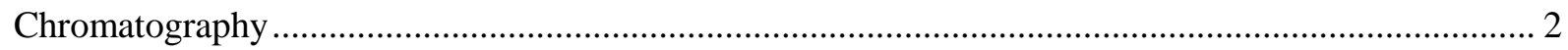

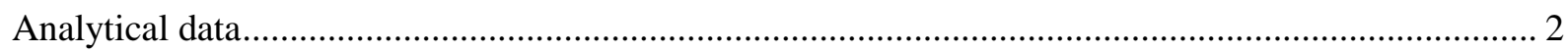

Typical procedure

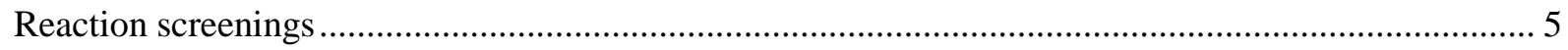

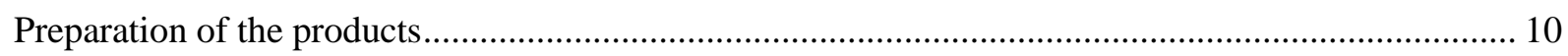

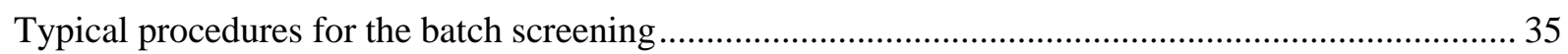

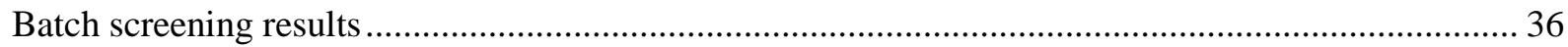

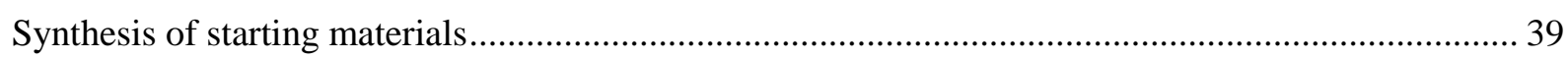

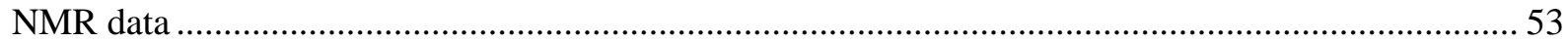




\section{General information}

\section{Solvents}

THF was continuously refluxed and freshly distilled from sodium benzophenone ketyl under nitrogen and stored over molecular sieves. Solvents for column chromatography were distilled prior to use.

\section{Reagents}

All reagents were obtained from commercial sources (Sigma-Aldrich, TCI, Merck, Acros Organics, Fluorochem and Apollo) and used without further purification.

$\boldsymbol{t}$ BuLi solution in hexane was purchased from Albemarle (Frankfurt) and the concentration was determined by titration against 1,10-phenanthroline in THF using $i \mathrm{PrOH}$.

\section{Chromatography}

Flash column chromatography was performed using $\mathrm{SiO}_{2} 60$ (0.040-0.063 mm, 230-400 mesh ASTM) from Merck. Thin layer chromatography (TLC) was performed using aluminum plates covered with $\mathrm{SiO}_{2}$ (Merck 60, F-254). Spots were visualized under UV light or with $\mathrm{KMnO}_{4}$.

\section{Analytical data}

Yields refer to isolated yields of analytically pure compounds estimated to be $>95 \%$ pure as determined by ${ }^{1} \mathrm{H}-\mathrm{NMR}\left(25^{\circ} \mathrm{C}\right)$ and capillary GC-analysis. NMR spectra were recorded on Bruker ARX 200, AC 300, WH 400 or AMX 600 instruments. Chemical shifts are reported as $\delta$-values in ppm relative to the deuterated solvent peak: $\mathrm{CDCl}_{3}(\delta \mathrm{H}: 7.26 ; \delta \mathrm{C}: 77.16)$. For the description of the observed signal multiplicities, the following abbreviations were used: $s$ (singlet), d (doublet), dd (doublet of doublets), $\mathrm{t}$ (triplet), q (quartet), quint (quintet), sext (sextet), sept (septet) and $\mathrm{m}$ (multiplet). Melting points are uncorrected and were measured on a Büchi B.540 apparatus. Infrared spectra were recorded from $4000-400 \mathrm{~cm}^{-1}$ on a Nicolet 510 FT-IR or a Perkin-Elmer 281 IR spectrometer. Samples were measured (Smiths Detection DuraSampl IR II Diamond ATR). The absorption bands are reported in wavenumbers $\left(\mathrm{cm}^{-1}\right)$. Gas chromatography (GC)-analyses were performed with instruments of the type HewlettPackard 6890 or 5890 Series II, using a column of the type HP 5 (Hewlett-Packard, 5\% phenylmethylpolysiloxane; length: $10 \mathrm{~m}$, diameter: $0.25 \mathrm{~mm}$, film thickness: $0.25 \mu \mathrm{m}$ ). The detection was accomplished using a flame ionization detector. Mass spectra (MS) and high resolution mass spectra (HRMS) were recorded on a Thermo Q Exactive GC Orbitrap. For the combination of gas chromatography with mass spectroscopic detection, a GC-MS of the type 
Hewlett-Packard 6890 / MSD 5793 networking was used (column: HP 5-MS, Hewlett-Packard; $5 \%$ phenylmethylpolysiloxane; length: $15 \mathrm{~m}$, diameter $0.25 \mathrm{~mm}$; film thickness: $0.25 \mu \mathrm{m}$ ).

\section{Typical procedure}

\section{General remarks on flow and subsequent batch quenching reactions}

Tetradecane $\left(n \mathrm{C}_{14} \mathrm{H}_{30}\right)$ was used as internal standard. All flasks were heat gun dried $\left(650{ }^{\circ} \mathrm{C}\right)$ under vacuum and backfilled with argon after cooling. Syringes, which were used to transfer reagents and solvents, were purged with argon three times prior to use. Batch quenching reactions were carried out with magnetic stirring. Flow reactions were performed on the commercially available flow system (Vapourtec E-series Integrated Flow Chemistry System with $3^{\text {rd }}$ Pump Kit). Hexane solutions of $t$ BuLi and THF solutions of the corresponding reagents were kept in flasks with rubber septa under an argon atmosphere during the reactions. All reactions were performed in coiled tube reactors. Coiled reactors were made from PFA or PTFE Teflon (I.D. $=0.8 \mathrm{~mm}$ or $0.25 \mathrm{~mm}$, O.D. $=1.6 \mathrm{~mm}$ ) tubing and T-pieces (I.D. $=0.5 \mathrm{~mm}$ ) were used as mixers. Prior to performing reactions, the systems were dried by flushing with dry THF or hexane (flow rate of all pumps: $1.00 \mathrm{~mL} / \mathrm{min}$; run-time: $30 \mathrm{~min}$ ). 


\section{Typical procedure 1 (TP1) using a Vapourtec E-series Integrated Flow Chemistry System}

(Scheme SI 1): Preparation of 1,2-Diphenylethan-1-ol (4a)

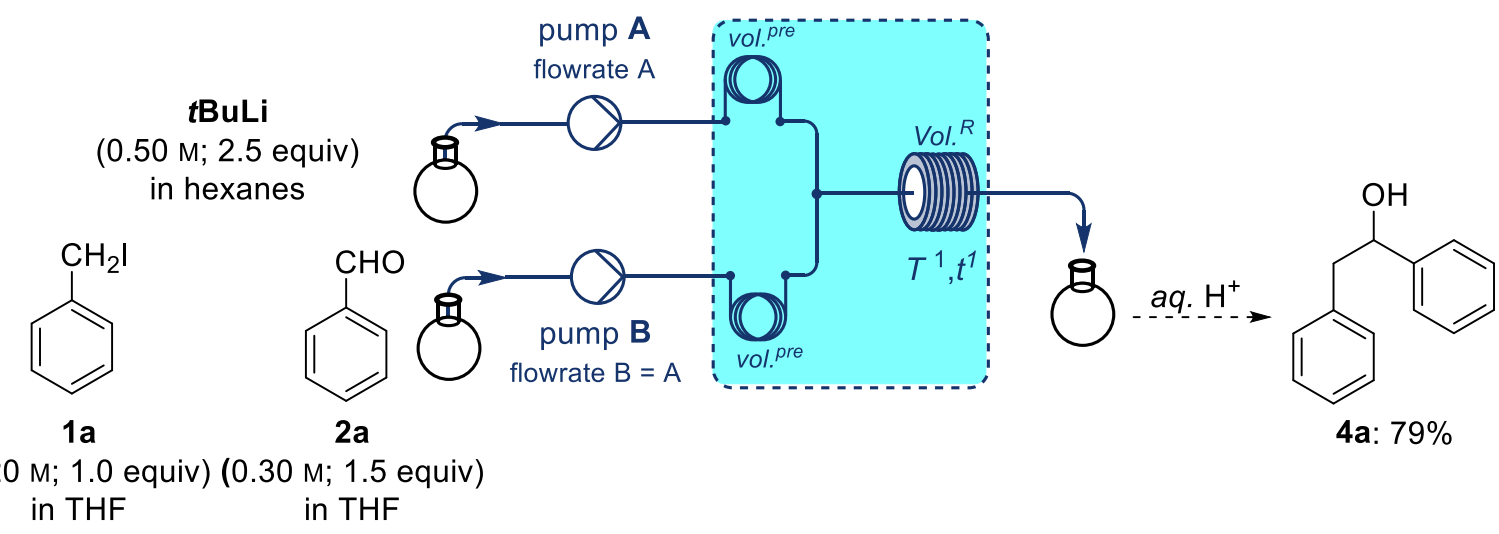

Scheme SI 1: Vapourtec E-series Integrated Flow Chemistry System for the iodine-lithium exchange of (hetero)benzylic substrates with $t \mathrm{BuLi}$ in the presence of various carbonyl compounds.

A solution of benzyl iodide (1a, $0.20 \mathrm{M}, 0.20 \mathrm{mmol}, 1.0$ equiv) and benzaldehyde (2a, $0.30 \mathrm{M}$, $0.30 \mathrm{mmol}, 1.5$ equiv) in THF (total volume: $1.0 \mathrm{~mL})$ and a solution of $t \mathrm{BuLi}(0.50 \mathrm{M}$ in hexane, $0.50 \mathrm{mmol}, 2.5$ equiv) were prepared. The precooled solutions were mixed with an overall $10 \mathrm{~mL} / \mathrm{min}$ flowrate in a T-mixer. The combined stream passed a $0.02 \mathrm{~mL}$ reactor tube $(0.1 \mathrm{~s}$, $-78{ }^{\circ} \mathrm{C}$ ) and was subsequently injected in an empty flask. Stirring was continued for $10 \mathrm{~min}$ at $25^{\circ} \mathrm{C}$ before sat. $a q$. $\mathrm{NH}_{4} \mathrm{Cl}$ solution was added to quench the reaction mixture. The aqueous phase was extracted three times with EtOAc $(3 \times 30 \mathrm{~mL})$ and the combined organic phases were dried over anhydrous $\mathrm{Na}_{2} \mathrm{SO}_{4}$ and filtrated. After removal of the solvent, flash chromatographical purification (silica gel, isohexane:EtOAc $=95: 5 \rightarrow 9: 1$ ) afforded the title compound $\mathbf{4 a}$ as a white amorphous solid (31 mg, $0.16 \mathrm{mmol}, 79 \%$ yield). 


\section{Reaction screenings}

Screening of reaction conditions:

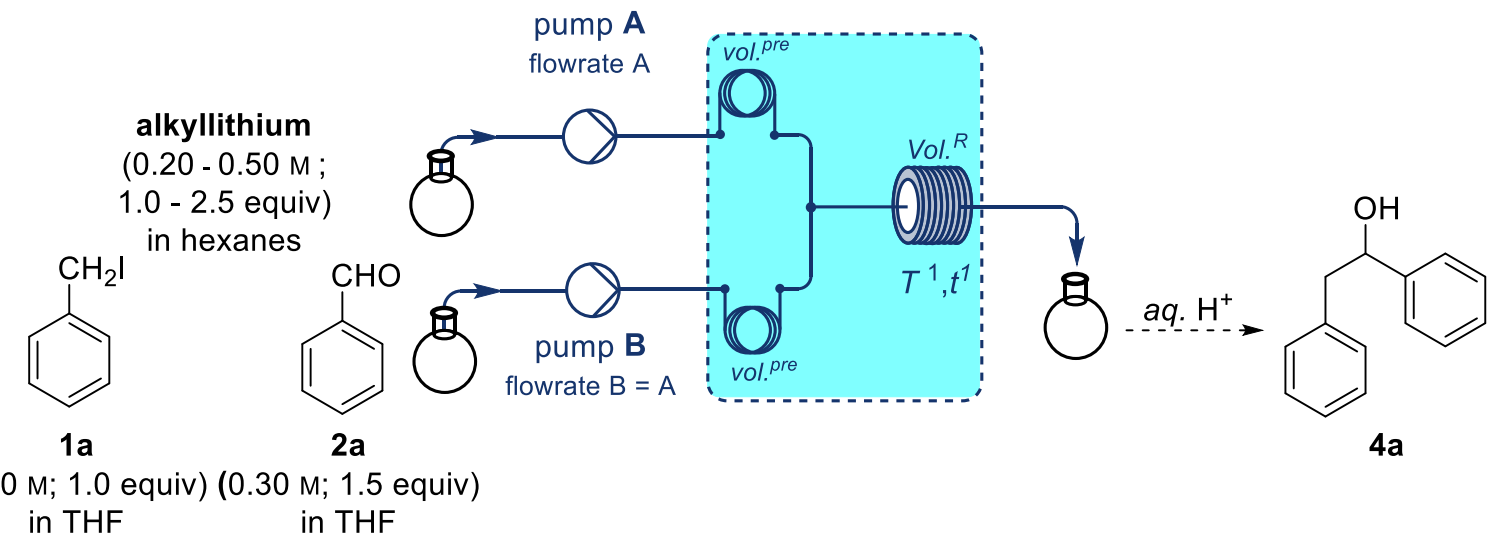

Scheme SI 2: General scheme for the optimization screening of $\mathbf{4 a}$.

All reactions were conducted with the same starting material solution. This results in a direct comparability of the values for the Wurtz-type product, conversion of starting material and desired product $\mathbf{4 a}$. The values for the addition of alkyllithium to the aldehyde $\mathbf{2 a}$ as well as the substitution product of alkyllithium at the benzyl iodide are not directly comparable. 
Table SI 1: Optimization screening of product 4a.

\begin{tabular}{|c|c|c|c|c|c|c|c|c|c|c|}
\hline entry & $\begin{array}{l}\text { base } \\
\text { [equiv] }\end{array}$ & $\begin{array}{c}\mathrm{t} \\
{[\mathrm{s}]}\end{array}$ & $\begin{array}{c}\text { vol }_{\text {reactor }} \\
{[\mathrm{mL}]}\end{array}$ & $\begin{array}{l}\text { flow rate } \\
{[\mathrm{mL} / \mathrm{min}]}\end{array}$ & $\begin{array}{c}\mathrm{T} \\
{\left[{ }^{\circ} \mathrm{C}\right]}\end{array}$ & $\mathrm{E}+\mathrm{Alk}^{[\mathrm{a}]}$ & $\mathrm{SM}+\mathrm{Alk}^{[\mathrm{a}]}$ & $\begin{array}{l}\text { Wurtz-type } \\
\text { side product }\end{array}$ & $\begin{array}{c}\text { conv. } \\
{[\%]}\end{array}$ & $\begin{array}{c}\text { GC- } \\
\text { yield } \\
{[\%]}\end{array}$ \\
\hline 1 & nBuLi (1.25) & 150 & 5 & 2 & 0 & 2.66 & 0.35 & 0.14 & 67 & 50 \\
\hline 2 & nBuLi (1.25) & 150 & 5 & 2 & -20 & 2.86 & 0.30 & 0.15 & 70 & 53 \\
\hline 3 & nBuLi (1.25) & 150 & 5 & 2 & -40 & 2.81 & 0.26 & 0.16 & 73 & 57 \\
\hline 4 & nBuLi (1.25) & 150 & 5 & 2 & -78 & 3.53 & 0.16 & 0.32 & 76 & 73 \\
\hline 5 & nBuLi (1.25) & 30 & 1 & 2 & 0 & 2.29 & 0.35 & 0.15 & 64 & 48 \\
\hline 6 & nBuLi (1.25) & 30 & 1 & 2 & -20 & 2.48 & 0.28 & 0.13 & 66 & 49 \\
\hline 7 & nBuLi (1.25) & 30 & 1 & 2 & -40 & 2.61 & 0.25 & 0.13 & 68 & 53 \\
\hline 8 & nBuLi (1.25) & 30 & 1 & 2 & -78 & 3.16 & 0.14 & 0.20 & 71 & 59 \\
\hline 9 & nBuLi (1.25) & 2.5 & 0.02 & 2 & 0 & 3.62 & 0.22 & 0.15 & 73 & 61 \\
\hline 10 & nBuLi (1.25) & 2.5 & 0.02 & 2 & -20 & 3.61 & 0.22 & 0.15 & 72 & 62 \\
\hline 11 & nBuLi (1.25) & 2.5 & 0.02 & 2 & -40 & 4.06 & 0.21 & 0.15 & 73 & 61 \\
\hline 12 & nBuLi (1.25) & 2.5 & 0.02 & 2 & -78 & 3.70 & 0.19 & 0.14 & 70 & 64 \\
\hline 13 & nBuLi (1.25) & 30 & 5 & 10 & 0 & 3.83 & 0.21 & 0.16 & 78 & 62 \\
\hline 14 & nBuLi (1.25) & 30 & 5 & 10 & -20 & 3.91 & 0.19 & 0.16 & 81 & 66 \\
\hline 15 & nBuLi (1.25) & 30 & 5 & 10 & -40 & 4.12 & 0.14 & 0.25 & 83 & 75 \\
\hline 16 & nBuLi (1.25) & 30 & 5 & 10 & -78 & 4.06 & 0.05 & 0.33 & 87 & 78 \\
\hline 17 & nBuLi (1.25) & 6 & 1 & 10 & 0 & 4.25 & 0.20 & 0.15 & 77 & 66 \\
\hline 18 & nBuLi (1.25) & 6 & 1 & 10 & -20 & 4.33 & 0.16 & 0.15 & 78 & 74 \\
\hline 19 & nBuLi (1.25) & 6 & 1 & 10 & -40 & 4.09 & 0.11 & 0.19 & 81 & 76 \\
\hline 20 & nBuLi (1.25) & 6 & 1 & 10 & -78 & 4.08 & 0.06 & 0.29 & 86 & 78 \\
\hline 21 & nBuLi (1.25) & 0.1 & 0.02 & 10 & 0 & 4.33 & 0.17 & 0.14 & 75 & 70 \\
\hline 22 & nBuLi (1.25) & 0.1 & 0.02 & 10 & -20 & 4.30 & 0.16 & 0.15 & 77 & 70 \\
\hline 23 & nBuLi (1.25) & 0.1 & 0.02 & 10 & -40 & 4.26 & 0.12 & 0.15 & 77 & 71 \\
\hline 24 & nBuLi (1.25) & 0.1 & 0.02 & 10 & -78 & 4.01 & 0.10 & 0.16 & 79 & 68 \\
\hline 25 & tBuLi (2.5) & 150 & 5 & 2 & 0 & 2.63 & 1.25 & 0.39 & 94 & 62 \\
\hline 26 & tBuLi (2.5) & 150 & 5 & 2 & -20 & 3.07 & 1.26 & 0.38 & 95 & 73 \\
\hline 27 & tBuLi (2.5) & 150 & 5 & 2 & -40 & 3.14 & 1.18 & 0.32 & 94 & 80 \\
\hline 28 & tBuLi (2.5) & 150 & 5 & 2 & -78 & 3.02 & 1.11 & 0.34 & 94 & 86 \\
\hline 29 & tBuLi (2.5) & 30 & 1 & 2 & 0 & 2.81 & 1.36 & 0.43 & 89 & 74 \\
\hline 30 & tBuLi (2.5) & 30 & 1 & 2 & -20 & 2.93 & 1.25 & 0.36 & 92 & 75 \\
\hline 31 & tBuLi (2.5) & 30 & 1 & 2 & -40 & 3.48 & 1.33 & 0.35 & 92 & 82 \\
\hline 32 & tBuLi (2.5) & 30 & 1 & 2 & -78 & 3.33 & 1.15 & 0.27 & 90 & 83 \\
\hline 33 & tBuLi (2.5) & 2.5 & 0.02 & 2 & 0 & 3.62 & 1.31 & 0.28 & 92 & 76 \\
\hline
\end{tabular}




\begin{tabular}{|c|c|c|c|c|c|c|c|c|c|}
\hline tBuLi (2.5) & 2.5 & 0.02 & 2 & -20 & 3.60 & 1.29 & 0.28 & 91 & 75 \\
\hline tBuLi (2.5) & 2.5 & 0.02 & 2 & -40 & 3.64 & 1.30 & 0.30 & 93 & 77 \\
\hline tBuLi (2.5) & 2.5 & 0.02 & 2 & -78 & 4.01 & 1.37 & 0.28 & 90 & 84 \\
\hline tBuLi (2.5) & 30 & 5 & 10 & 0 & 3.61 & 1.28 & 0.38 & 95 & 77 \\
\hline tBuLi (2.5) & 30 & 5 & 10 & -20 & 3.47 & 1.25 & 0.35 & 93 & 75 \\
\hline tBuLi (2.5) & 30 & 5 & 10 & -40 & 3.35 & 1.11 & 0.25 & 96 & 80 \\
\hline tBuLi (2.5) & 30 & 5 & 10 & -78 & 3.64 & 1.05 & 0.35 & 97 & 97 \\
\hline tBuLi (2.5) & 6 & 1 & 10 & 0 & 3.29 & 1.24 & 0.36 & 93 & 75 \\
\hline tBuLi (2.5) & 6 & 1 & 10 & -20 & 3.58 & 1.26 & 0.37 & 95 & 72 \\
\hline tBuLi (2.5) & 6 & 1 & 10 & -40 & 3.68 & 1.26 & 0.34 & 95 & 82 \\
\hline tBuLi (2.5) & 6 & 1 & 10 & -78 & 3.59 & 1.27 & 0.35 & 95 & 80 \\
\hline tBuLi (2.5) & 0.1 & 0.02 & 10 & 0 & 3.70 & 1.29 & 0.32 & 94 & 81 \\
\hline tBuLi (2.5) & 0.1 & 0.02 & 10 & -20 & 3.65 & 1.27 & 0.32 & 94 & 81 \\
\hline tBuLi (2.5) & 0.1 & 0.02 & 10 & -40 & 4.13 & 1.29 & 0.36 & 96 & 87 \\
\hline tBuLi (2.5) & 0.1 & 0.02 & 10 & -78 & 4.03 & 1.22 & 0.34 & 94 & 87 \\
\hline tBuLi (2.5) ${ }^{[a]}$ & 0.1 & 0.02 & 10 & -78 & 0.27 & 0.20 & 1.65 & 100 & 13 \\
\hline tBuLi (2.5) $)^{[\mathrm{b}]}$ & 0.1 & 0.02 & 10 & -78 & 0.00 & 0.11 & 1.78 & 100 & 10 \\
\hline sBuLi (1.1) & 0.1 & 0.02 & 10 & -78 & & & & 75 & 59 \\
\hline$n \mathrm{HexLi}(1.1)$ & 0.1 & 0.02 & 10 & -78 & & & & 68 & 57 \\
\hline $\begin{array}{c}\text { neopentyl Li } \\
(1.1)\end{array}$ & 0.1 & 0.02 & 10 & -78 & & & & 83 & 62 \\
\hline
\end{tabular}

${ }^{\text {[a] }}$ The values were calculated according to $x=\frac{\text { GC value }}{\text { Standard value }}{ }^{[b]}$ TMEDA (2.5 equiv) was added to the $t$ BuLi solution. ${ }^{[c]}$ PMDTA (2.5 equiv) was added to the $t$ BuLi solution.<smiles>OC(Cl)c1ccccc1</smiles>

alkyllithium addition to electrophile (E+Alk)

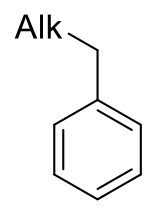

alkyllithium substitution of iodide (SM+Alk)<smiles>c1ccc(CCc2ccccc2)cc1</smiles>

Wurtz-type side product<smiles>OC(Cc1ccccc1)c1ccccc1</smiles>

product (4a) 
Screening of different additives:

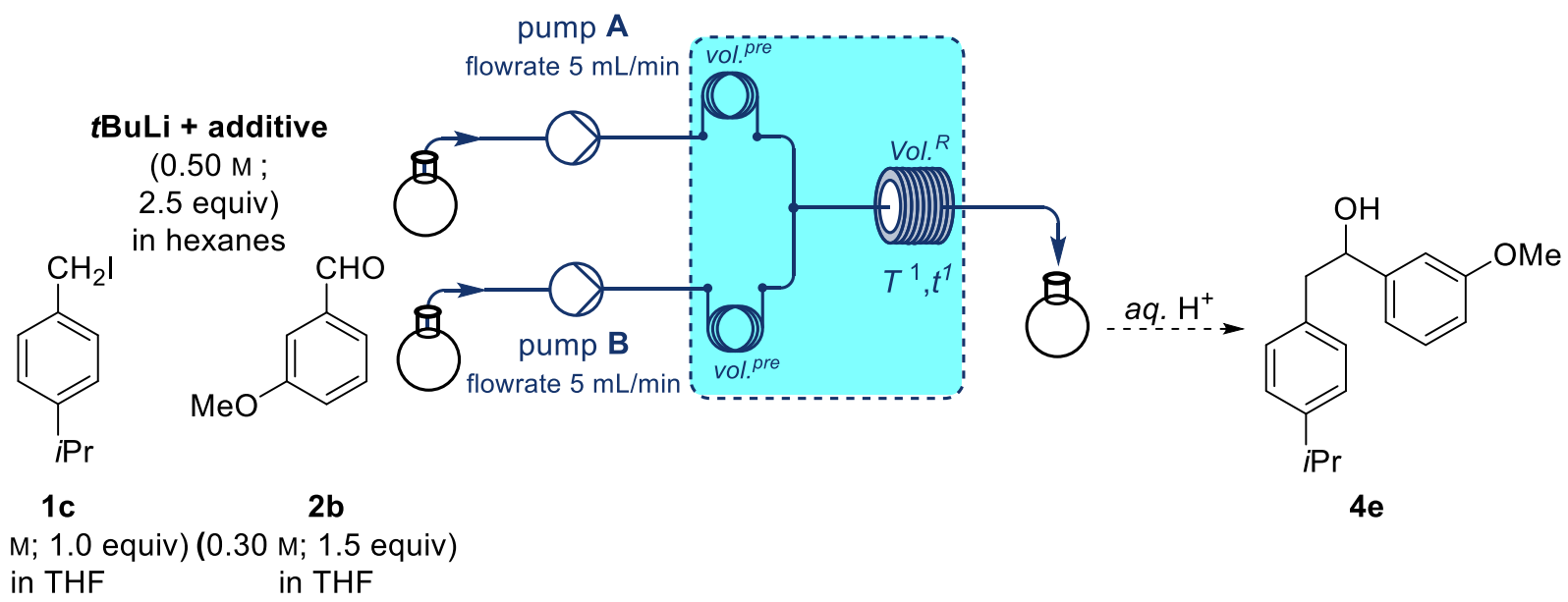

Scheme SI 3: General scheme for the additive screening of Barbier-type reaction of $\mathbf{1 c}$ and 2b affording product $\mathbf{4 e}$.

Table SI 2: Additive screening of Barbier-type reaction of $\mathbf{1 c}$ and $\mathbf{2 b}$ affording product $\mathbf{4 e .}$

\begin{tabular}{|c|c|c|c|c|c|c|c|c|c|}
\hline & base & & $\mathrm{t}$ & $\mathrm{T}$ & A & Al & Wurtz-type & conv & C-yielc \\
\hline entry & [equiv] & additıve & [s] & {$\left[{ }^{\circ} \mathrm{C}\right]$} & E+AIK & $S I V I+A I K^{[a]}$ & side product ${ }^{[a]}$ & {$[\%]$} & [\%] \\
\hline 1 & tBuLi (2.5) & TMEDA & 0.1 & -20 & 0.57 & 0.09 & 3.74 & 100 & 4 \\
\hline 2 & tBuLi (2.5) & TMEDA & 0.1 & -40 & 0.69 & 0.10 & 3.68 & 100 & 4 \\
\hline 3 & tBuLi (2.5) & TMEDA & 0.1 & -78 & 0.57 & 0.09 & 3.74 & 100 & 4 \\
\hline 4 & tBuLi (2.5) & PMDTA & 0.1 & -20 & 0.15 & 0.01 & 3.93 & 100 & 2 \\
\hline 5 & tBuLi (2.5) & PMDTA & 0.1 & -40 & 0.13 & 0.01 & 4.15 & 100 & 3 \\
\hline 6 & tBuLi (2.5) & PMDTA & 0.1 & -78 & 0.13 & 0.00 & 4.39 & 100 & 3 \\
\hline 7 & tBuLi (2.5) & - & 0.1 & -78 & 0.74 & 1.91 & 0.71 & 100 & 71 \\
\hline
\end{tabular}

[a] The values were calculated according to $x=\frac{\mathrm{GC} \text { value }}{\text { Standard value }}$. 


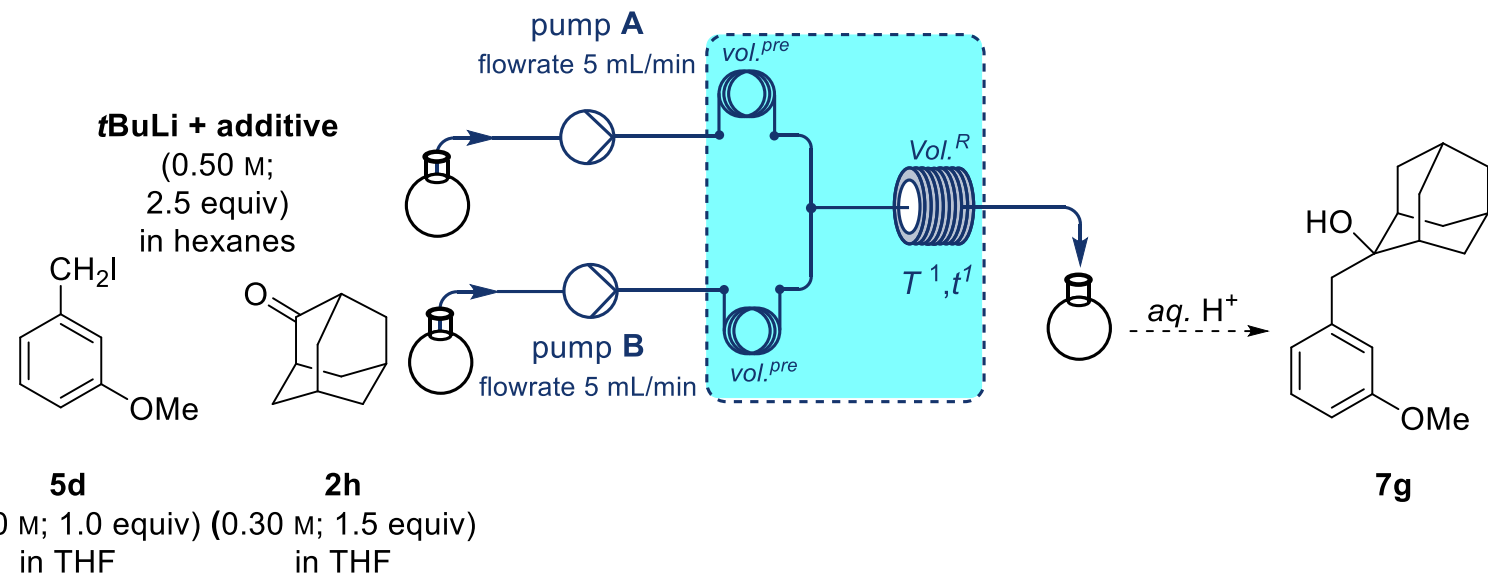

Scheme SI 4: General scheme for the additive screening of Barbier-type reaction of $\mathbf{5 d}$ and $\mathbf{2 h}$ affording product $\mathbf{7 g}$.

Table SI 3: Additive screening of Barbier-type reaction of $\mathbf{5 d}$ and $\mathbf{2 h}$ affording product $\mathbf{7 g}$.

\begin{tabular}{|c|c|c|c|c|c|c|c|c|c|}
\hline \multirow{2}{*}{ entry } & \multirow{2}{*}{$\begin{array}{l}\text { base } \\
\text { [equiv] }\end{array}$} & \multirow{2}{*}{ additive } & \multirow{2}{*}{$\begin{array}{c}\mathrm{t} \\
{[\mathrm{s}]}\end{array}$} & \multirow{2}{*}{$\begin{array}{c}\mathrm{T} \\
{\left[{ }^{\circ} \mathrm{C}\right]}\end{array}$} & \multirow{2}{*}{$\mathrm{E}+\mathrm{Alk}^{[\mathrm{a}]}$} & \multirow{2}{*}{$\mathrm{SM}+\mathrm{Alk}^{[\mathrm{a}]}$} & \multirow{2}{*}{$\begin{array}{l}\text { Wurtz-type } \\
\text { side product }{ }^{[a]}\end{array}$} & \multicolumn{2}{|c|}{ conv.GC-yield } \\
\hline & & & & & & & & [\%] & {$[\%]$} \\
\hline 1 & tBuLi (2.5) & TMEDA & 0.1 & -20 & 0.10 & 0.07 & 2.46 & 100 & 12 \\
\hline 2 & tBuLi (2.5) & TMEDA & 0.1 & -40 & 0.12 & 0.07 & 2.71 & 100 & 13 \\
\hline 3 & tBuLi (2.5) & TMEDA & 0.1 & -78 & 0.14 & 0.07 & 2.72 & 100 & 11 \\
\hline 4 & tBuLi (2.5) & PMDTA & 0.1 & -20 & 0.03 & 0.01 & 2.33 & 100 & 5 \\
\hline 5 & tBuLi (2.5) & PMDTA & 0.1 & -40 & 0.04 & 0.00 & 2.32 & 99 & 5 \\
\hline 6 & tBuLi (2.5) & PMDTA & 0.1 & -78 & 0.03 & 0.00 & 2.22 & 98 & 5 \\
\hline 7 & tBuLi (2.5) & - & 0.1 & -78 & 0.09 & 1.28 & 0.98 & 96 & 85 \\
\hline
\end{tabular}




\section{Preparation of the products}

\section{1,2-Diphenylethan-1-ol (4a)}<smiles>OC(Cc1ccccc1)c1ccccc1</smiles>

According to the TP1, a solution of benzyl iodide (1a, $0.20 \mathrm{M}, 0.20 \mathrm{mmol}, 1.0$ equiv) and benzaldehyde (2a, $0.30 \mathrm{M}, 0.30 \mathrm{mmol}, 1.5$ equiv) in THF (total volume: $1.0 \mathrm{~mL}$ ) and a solution of $t \mathrm{BuLi}$ ( $0.50 \mathrm{M}$ in hexane, $0.50 \mathrm{mmol}, 2.5$ equiv) were prepared. The precooled solutions were mixed with an overall $10 \mathrm{~mL} / \mathrm{min}$ flowrate in a T-mixer. The combined stream passed a $0.02 \mathrm{~mL}$ reactor tube $\left(0.1 \mathrm{~s},-78^{\circ} \mathrm{C}\right)$ and was subsequently injected in an empty flask. Stirring was continued for $10 \mathrm{~min}$ at $25^{\circ} \mathrm{C}$ before sat. $a q . \mathrm{NH}_{4} \mathrm{Cl}$ solution was added to quench the reaction mixture. The aqueous phase was extracted three times with EtOAc $(3 \times 30 \mathrm{~mL})$ and the combined organic phases were dried over anhydrous $\mathrm{Na}_{2} \mathrm{SO}_{4}$ and filtrated. After removal of the solvent, flash chromatographical purification (silica gel, isohexane:EtOAc $=95: 5 \rightarrow$ 9:1) afforded the title compound $\mathbf{4 a}$ as a white amorphous solid (31 $\mathrm{mg}, 0.16 \mathrm{mmol}, 79 \%$ yield).

In addition, a convenient scale-up of the reaction according to TP1 was demonstrated. A solution of benzyl iodide (1a, $0.20 \mathrm{M}, 4.00 \mathrm{mmol}, 1.0$ equiv) and benzaldehyde (2a, $0.30 \mathrm{M}$, $6.00 \mathrm{mmol}, 1.5$ equiv) in THF (total volume: $20.0 \mathrm{~mL})$ and a solution of $t \mathrm{BuLi}(0.50 \mathrm{M}$ in hexane, $10.0 \mathrm{mmol}, 2.5$ equiv) were prepared. The precooled solutions were mixed with an overall $10 \mathrm{~mL} / \mathrm{min}$ flowrate in a T-mixer. The combined stream passed a $0.02 \mathrm{~mL}$ reactor tube $\left(0.1 \mathrm{~s},-78^{\circ} \mathrm{C}\right)$ and was subsequently injected in an empty flask. Stirring was continued for $10 \mathrm{~min}$ at $25^{\circ} \mathrm{C}$ before sat. aq. $\mathrm{NH}_{4} \mathrm{Cl}$ solution was added to quench the reaction mixture. The aqueous phase was extracted three times with EtOAc $(3 \times 100 \mathrm{~mL})$ and the combined organic phases were dried over anhydrous $\mathrm{Na}_{2} \mathrm{SO}_{4}$ and filtrated. After removal of the solvent, flash chromatographical purification (silica gel, isohexane:EtOAc $=95: 5 \rightarrow 9: 1$ ) afforded the title compound $4 \mathbf{a}$ as a white amorphous solid (619 mg, $3.12 \mathrm{mmol}$, $78 \%$ yield).

${ }^{1}$ H-NMR (400 MHz, CDCl3): $\delta / \mathrm{ppm}=7.42-7.15(\mathrm{~m}, 10 \mathrm{H}), 4.91(\mathrm{dd}, J=8.5,4.9 \mathrm{~Hz}, 1 \mathrm{H})$, $3.13-2.88(\mathrm{~m}, 2 \mathrm{H}), 1.97(\mathrm{~s}, 1 \mathrm{H})$.

${ }^{13}$ C-NMR (100 MHz, CDCl3): $\delta$ / ppm = 143.9, 138.2, 129.6 (2C), 128.7 (2C), 128.6 (2C), $127.8,126.8,126.0(2 \mathrm{C}), 75.5,46.2$.

IR (Diamond-ATR, neat): $\tilde{v} / \mathrm{cm}^{-1}=3294,3026,2922,2855,1495,1453,1445,1072,1039$, 1026, 1016, 952, 778, 760, 741, 696.

MS (EI, 70 eV): $m / z(\%)=107$ (59), 92 (100), 91 (41), 79 (71), 77 (23).

HRMS (EI-orbitrap): $m / z:$ : $\mathrm{M}-\mathrm{H}_{2} \mathrm{O}$ ] calcd for $\mathrm{C}_{14} \mathrm{H}_{12}$ 180.0939; Found 180.0930. 
m.p. $\left({ }^{\circ} \mathbf{C}\right): 63.9-66.4$.

\section{1-(3-Methoxyphenyl)-2-phenylethan-1-ol (4b)}<smiles>COc1cccc(C(O)Cc2ccccc2)c1</smiles>

According to the TP1, a solution of benzyl iodide (1a, $0.20 \mathrm{M}, 0.20 \mathrm{mmol}, 1.0$ equiv) and $p$ anisaldehyde (2b, $0.30 \mathrm{M}, 0.30 \mathrm{mmol}, 1.5$ equiv) in THF (total volume: $1.0 \mathrm{~mL}$ ) and a solution of $t \mathrm{BuLi}$ ( $0.50 \mathrm{M}$ in hexane, $0.50 \mathrm{mmol}, 2.5$ equiv) were prepared. The precooled solutions were mixed with an overall $10 \mathrm{~mL} / \mathrm{min}$ flowrate in a T-mixer. The combined stream passed a $0.02 \mathrm{~mL}$ reactor tube $\left(0.1 \mathrm{~s},-78^{\circ} \mathrm{C}\right)$ and was subsequently injected in an empty flask. Stirring was continued for $10 \mathrm{~min}$ at $25{ }^{\circ} \mathrm{C}$ before sat. $a q . \mathrm{NH}_{4} \mathrm{Cl}$ solution was added to quench the reaction mixture. The aqueous phase was extracted three times with EtOAc $(3 \times 30 \mathrm{~mL})$ and the combined organic phases were dried over anhydrous $\mathrm{Na}_{2} \mathrm{SO}_{4}$ and filtrated. After removal of the solvent, flash chromatographical purification (silica gel, isohexane:EtOAc $=95: 5 \rightarrow$ 9:1) afforded the title compound $\mathbf{4 b}$ as a colorless amorphous solid (28 $\mathrm{mg}, 0.13 \mathrm{mmol}, 63 \%$ yield).

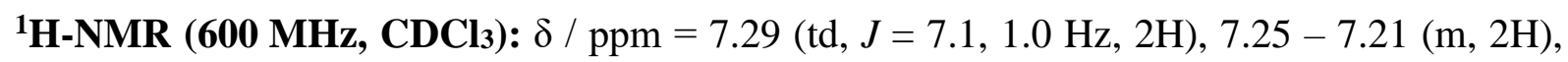
$7.21-7.18(\mathrm{~m}, 2 \mathrm{H}), 6.94-6.89(\mathrm{~m}, 2 \mathrm{H}), 6.83-6.79(\mathrm{~m}, 1 \mathrm{H}), 4.87(\mathrm{dd}, J=8.6,4.7 \mathrm{~Hz}, 1 \mathrm{H})$, $3.79(\mathrm{~d}, J=0.8 \mathrm{~Hz}, 3 \mathrm{H}), 3.06-2.93(\mathrm{~m}, 2 \mathrm{H}), 1.96(\mathrm{~s}, 1 \mathrm{H})$.

${ }^{13}$ C-NMR (150 MHz, CDCl3): $\delta$ / ppm = 159.8, 145.7, 138.1, 129.7 (2C), 129.6, 128.7 (2C), $126.8,118.3,113.3,111.4,75.4,55.4,46.2$.

IR (Diamond-ATR, neat): $\tilde{v} / \mathrm{cm}^{-1}=3294,3026,2922,2855,1495,1453,1445,1316,1072$, 1039, 1026, 1016, 952, 917, 778, 760, 741, 696.

MS (EI, 70 eV): m/z (\%) = 137 (16), 136 (16), 109 (12), 71 (26), 70 (10), 71 (17), 57 (30), 56 (19), 45 (14), 43 (100), 42 (37), 41 (27).

HRMS (EI-orbitrap): $m / z$ : [M] calcd for $\mathrm{C}_{15} \mathrm{H}_{16} \mathrm{O}_{2}$ 228.1150; Found 228.1126. m.p. $\left({ }^{\circ} \mathbf{C}\right): 72.4-74.8$. 


\section{2-(4-(Tert-butyl)phenyl)-1-phenylethan-1-ol (4c)}<smiles>CC(C)(C)c1ccc(CC(O)c2ccccc2)cc1</smiles>

According to the TP1, a solution of 1-(tert-butyl)-4-(iodomethyl)benzene $(\mathbf{1 b}, 0.20 \mathrm{M}$, $0.20 \mathrm{mmol}, 1.0$ equiv) and benzaldehyde (2a, $0.30 \mathrm{M}, 0.30 \mathrm{mmol}, 1.5$ equiv) in THF (total volume: $1.0 \mathrm{~mL})$ and a solution of $t \mathrm{BuLi}(0.50 \mathrm{M}$ in hexane, $0.50 \mathrm{mmol}, 2.5$ equiv) were prepared. The precooled solutions were mixed with an overall $10 \mathrm{~mL} / \mathrm{min}$ flowrate in a T-mixer. The combined stream passed a $0.02 \mathrm{~mL}$ reactor tube $\left(0.1 \mathrm{~s},-78^{\circ} \mathrm{C}\right)$ and was subsequently injected in an empty flask. Stirring was continued for $10 \mathrm{~min}$ at $25^{\circ} \mathrm{C}$ before sat. $a q . \mathrm{NH}_{4} \mathrm{Cl}$ solution was added to quench the reaction mixture. The aqueous phase was extracted three times with EtOAc $(3 \times 30 \mathrm{~mL})$ and the combined organic phases were dried over anhydrous $\mathrm{Na}_{2} \mathrm{SO}_{4}$ and filtrated. After removal of the solvent, flash chromatographical purification (silica gel, isohexane:EtOAc $=95: 5 \rightarrow 9: 1)$ afforded the title compound $\mathbf{4 c}$ as a white amorphous solid (33 $\mathrm{mg}, 0.13 \mathrm{mmol}, 65 \%$ yield).

${ }^{1}$ H-NMR (400 MHz, CDCl3): $\delta$ / ppm = 7.43 - 7.38 (m, 3H), 7.38 - 7.34 (m, 3H), 7.33 - 7.27 $(\mathrm{m}, 1 \mathrm{H}), 7.21-7.15(\mathrm{~m}, 2 \mathrm{H}), 4.90(\mathrm{dd}, J=9.2,4.1 \mathrm{~Hz}, 1 \mathrm{H}), 3.10-2.87(\mathrm{~m}, 2 \mathrm{H}), 1.92(\mathrm{~s}, 1 \mathrm{H})$, $1.33(\mathrm{~s}, 9 \mathrm{H})$.

${ }^{13}$ C-NMR (100 MHz, CDCl3): $\delta$ / ppm = 149.7, 144.1, 135.2, 129.3 (2C), 128.6 (2C), 127.7, 126.0 (2C), 125.7 (2C), 75.4, 45.8, 34.6, 31.5 (3C).

IR (Diamond-ATR, neat): $\tilde{v} / \mathrm{cm}^{-1}=3560,2951,1509,1493,1453,1360,1269,1049,1028$, 910, 817, 755, 731, 699.

MS (EI, 70 eV): $m / z(\%)=148$ (24), 134 (11), 133 (100), 117 (12), 107 (14), 105 (11), 79 (19).

HRMS (EI-orbitrap): $m / z:$ : $\mathrm{M}-\mathrm{H}_{2} \mathrm{O}$ ] calcd for $\mathrm{C}_{18} \mathrm{H}_{20} 236.1565$; Found 236.1557.

m.p. $\left({ }^{\circ} \mathbf{C}\right): 58.0-60.9$. 


\section{2-(4-(Tert-butyl)phenyl)-1-(4-methoxyphenyl)ethan-1-ol (4d)}<smiles>COc1ccc(C(O)Cc2ccc(C(C)(C)C)cc2)cc1</smiles>

According to the TP1, a solution of 1-(tert-butyl)-4-(iodomethyl)benzene $(\mathbf{1 b}, 0.20 \mathrm{M}$, $0.20 \mathrm{mmol}, 1.0$ equiv) and $p$-anisaldehyde $(2 \mathrm{c}, 0.30 \mathrm{M}, 0.30 \mathrm{mmol}, 1.5$ equiv) in THF (total volume: $1.0 \mathrm{~mL})$ and a solution of $t \mathrm{BuLi}(0.50 \mathrm{M}$ in hexane, $0.50 \mathrm{mmol}, 2.5$ equiv) were prepared. The precooled solutions were mixed with an overall $10 \mathrm{~mL} / \mathrm{min}$ flowrate in a T-mixer. The combined stream passed a $0.02 \mathrm{~mL}$ reactor tube $\left(0.1 \mathrm{~s},-78^{\circ} \mathrm{C}\right)$ and was subsequently injected in an empty flask. Stirring was continued for $10 \mathrm{~min}$ at $25{ }^{\circ} \mathrm{C}$ before sat. aq. $\mathrm{NH}_{4} \mathrm{Cl}$ solution was added to quench the reaction mixture. The aqueous phase was extracted three times with EtOAc $(3 \times 30 \mathrm{~mL})$ and the combined organic phases were dried over anhydrous $\mathrm{Na}_{2} \mathrm{SO}_{4}$ and filtrated. After removal of the solvent, flash chromatographical purification (silica gel, isohexane:EtOAc $=95: 5 \rightarrow 9: 1)$ afforded the title compound $\mathbf{4 d}$ as a white amorphous solid (32 $\mathrm{mg}, 0.11 \mathrm{mmol}, 56 \%$ yield).

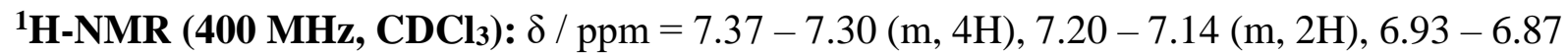
(m, 2H), 4.85 (ddd, $J=8.8,4.5,2.2 \mathrm{~Hz}, 1 \mathrm{H}), 3.82(\mathrm{~s}, 3 \mathrm{H}), 3.05-2.88(\mathrm{~m}, 2 \mathrm{H}), 1.93(\mathrm{~d}, J=2.6$ $\mathrm{Hz}, 1 \mathrm{H}), 1.32$ (s, 9H).

${ }^{13}$ C-NMR (100 MHz, CDCl3): $\delta$ / ppm = 159.2, 149.6, 136.3, 135.3, 129.2 (2C), 127.3 (2C), 125.6 (2C), 113.9 (2C), 75.0, 55.4, 45.7, 34.6, 31.5 (3C).

IR (Diamond-ATR, neat): $\tilde{v} / \mathrm{cm}^{-1}=3557,2952,1608,1509,1249,1178,1106,1047,1028$, $878,829,814$.

MS (EI, 70 eV): $m / z(\%)=266$ (11), 251 (13), 148 (11), 137 (52), 88 (16), 73 (13), 70 (16), 61 (20), 45 (13), 43 (100).

HRMS (EI-orbitrap): $m / z$ : [M] calcd for $\mathrm{C}_{19} \mathrm{H}_{24} \mathrm{O}_{2}$ 284.1776; Found 284.1751.

m.p. $\left({ }^{\circ} \mathbf{C}\right): 66.2-67.6$. 


\section{2-(4-Isopropylphenyl)-1-(3-methoxyphenyl)ethan-1-ol (4e)}<smiles>COc1cccc(C(O)Cc2ccc(C(C)C)cc2)c1</smiles>

According to the TP1, a solution of 1-(iodomethyl)-4-isopropylbenzene (1c, $0.20 \mathrm{M}$, $0.20 \mathrm{mmol}, 1.0$ equiv) and $m$-anisaldehyde $(\mathbf{2 b}, 0.30 \mathrm{M}, 0.30 \mathrm{mmol}, 1.5$ equiv) in THF (total volume: $1.0 \mathrm{~mL})$ and a solution of $t \mathrm{BuLi}(0.50 \mathrm{M}$ in hexane, $0.50 \mathrm{mmol}, 2.5$ equiv) were prepared. The precooled solutions were mixed with an overall $10 \mathrm{~mL} / \mathrm{min}$ flowrate in a T-mixer. The combined stream passed a $0.02 \mathrm{~mL}$ reactor tube $\left(0.1 \mathrm{~s},-20^{\circ} \mathrm{C}\right)$ and was subsequently injected in an empty flask. Stirring was continued for 10 min at $25{ }^{\circ} \mathrm{C}$ before sat. aq. $\mathrm{NH}_{4} \mathrm{Cl}$ solution was added to quench the reaction mixture. The aqueous phase was extracted three times with EtOAc $(3 \times 30 \mathrm{~mL})$ and the combined organic phases were dried over anhydrous $\mathrm{Na}_{2} \mathrm{SO}_{4}$ and filtrated. After removal of the solvent, flash chromatographical purification (silica gel, isohexane:EtOAc $=95: 5 \rightarrow$ 9:1) afforded the title compound $\mathbf{4 e}$ as a colorless oil $(30 \mathrm{mg}, 0.14$ mmol, $71 \%$ yield).

${ }^{1}$ H-NMR (400 MHz, CDCl3): $\delta$ / ppm = 7.25 - 7.21 (m, 1H), 7.18 - 7.10 (m, 4H), 6.96 - 6.91 $(\mathrm{m}, 1 \mathrm{H}), 6.90(\mathrm{t}, J=2.1 \mathrm{~Hz}, 1 \mathrm{H}), 6.80(\mathrm{ddd}, J=8.2,2.6,1.0 \mathrm{~Hz}, 1 \mathrm{H}), 4.83(\mathrm{dd}, J=9.1,4.3 \mathrm{~Hz}$, $1 \mathrm{H}), 3.77(\mathrm{~s}, 3 \mathrm{H}), 3.02-2.82(\mathrm{~m}, 3 \mathrm{H}), 1.95(\mathrm{~d}, J=2.5 \mathrm{~Hz}, 1 \mathrm{H}), 1.22(\mathrm{~d}, J=6.9 \mathrm{~Hz}, 6 \mathrm{H})$.

${ }^{13}$ C-NMR (100 MHz, CDCl $): \delta /$ ppm = 159.8, 147.4, 145.8, 135.4, 129.6, 129.5 (2C), 126.8 (2C), 118.3, 113.3, 111.3, 75.4, 55.4, 45.9, 33.9, $24.2(2 \mathrm{C})$.

IR (Diamond-ATR, neat): $\tilde{v} / \mathrm{cm}^{-1}=3421,2958,2870,2835,1601,1586,1513,1488,1463$, 1456, 1434, 1383, 1362, 1339, 1318, 1257, 1190, 1149, 1040, 1020, 997, 876, 864, 842, 815, 783, 763, 727, 695 .

MS (EI, 70 eV): m/z (\%) = 137 (25), 134 (72), 120 (10), 119 (100), 117 (12), 109 (58), 94 (18), 91 (12).

HRMS (EI-orbitrap): $m / z:[\mathrm{M}-\mathrm{OH}]^{+}$calcd for $\mathrm{C}_{18} \mathrm{H}_{21} \mathrm{O}^{+}$253.1587; Found 253.1589. 


\section{1-Cyclohexyl-2-(4-isopropylphenyl)ethan-1-ol (4f)}<smiles>CC(C)c1ccc(CC(O)C2CCCCC2)cc1</smiles>

According to the TP1, a solution of 1-(iodomethyl)-4-isopropylbenzene (1c, $0.20 \mathrm{M}$, $0.20 \mathrm{mmol}, 1.0$ equiv) and cyclohexanecarboxaldehyde (2d, $0.30 \mathrm{M}, 0.30 \mathrm{mmol}, 1.5$ equiv) in THF (total volume: $1.0 \mathrm{~mL}$ ) and a solution of $t \mathrm{BuLi}(0.50 \mathrm{M}$ in hexane, $0.50 \mathrm{mmol}, 2.5$ equiv) were prepared. The precooled solutions were mixed with an overall $10 \mathrm{~mL} / \mathrm{min}$ flowrate in a Tmixer. The combined stream passed a $0.02 \mathrm{~mL}$ reactor tube $\left(0.1 \mathrm{~s}, 25^{\circ} \mathrm{C}\right)$ and was subsequently injected in an empty flask. Stirring was continued for $10 \mathrm{~min}$ at $25^{\circ} \mathrm{C}$ before sat. aq. $\mathrm{NH}_{4} \mathrm{Cl}$ solution was added to quench the reaction mixture. The aqueous phase was extracted three times with EtOAc $(3 \times 30 \mathrm{~mL})$ and the combined organic phases were dried over anhydrous $\mathrm{Na}_{2} \mathrm{SO}_{4}$ and filtrated. After removal of the solvent, flash chromatographical purification (silica gel, isohexane:EtOAc $=95: 5 \rightarrow$ 9:1) afforded the title compound $\mathbf{4 f}$ as a white amorphous solid (38 $\mathrm{mg}, 0.15 \mathrm{mmol}, 76 \%$ yield).

${ }^{1}$ H-NMR (400 MHz, CDCl3): $\delta / \mathrm{ppm}=7.21-7.11(\mathrm{~m}, 4 \mathrm{H}), 3.56(\mathrm{ddt}, J=9.0,5.7,3.0 \mathrm{~Hz}$, $1 \mathrm{H}), 2.94-2.81(\mathrm{~m}, 2 \mathrm{H}), 2.56(\mathrm{dd}, J=13.7,9.7 \mathrm{~Hz}, 1 \mathrm{H}), 1.92(\mathrm{dtt}, J=12.6,3.3,1.7 \mathrm{~Hz}, 1 \mathrm{H})$, $1.84-1.73(\mathrm{~m}, 3 \mathrm{H}), 1.73-1.64(\mathrm{~m}, 1 \mathrm{H}), 1.50-1.38(\mathrm{~m}, 2 \mathrm{H}), 1.34-1.03(\mathrm{~m}, 11 \mathrm{H})$.

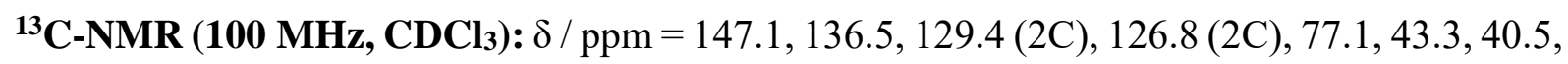
$33.9,29.4,28.2,26.7,26.5,26.3,24.2,24.2$.

IR (Diamond-ATR, neat): $\tilde{v} / \mathrm{cm}^{-1}=3314,2957,2928,2919,2891,2844,1514,1445,1418$, 1335, 1293, 1105, 1084, 1057, 1030, 1005, 891, 866, 847, 838, 812.

MS (EI, 70 eV): $m / z(\%)=134$ (64), 119 (60), 95 (19), 91 (11), 88 (14), 73 (11), 70 (15), 61 (26), 39 (13).

HRMS (EI-orbitrap): $m / z$ : [M] calcd for $\mathrm{C}_{17} \mathrm{H}_{26} \mathrm{O}$ 246.1984; Found 246.1976.

m.p. $\left({ }^{\circ} \mathbf{C}\right): 54.6-55.9$. 


\section{1-(4-Isopropylphenyl)-2-phenylpropan-2-ol (4g)}<smiles>CC(C)c1ccc(CC(O)(c2ccccc2)c2ccccc2)cc1</smiles>

According to the TP1, a solution of 1-(iodomethyl)-4-isopropylbenzene (1c, $0.20 \mathrm{M}$, $0.20 \mathrm{mmol}, 1.0$ equiv) and acetophenone $(2 \mathrm{e}, 0.30 \mathrm{M}, 0.30 \mathrm{mmol}, 1.5$ equiv) in THF (total volume: $1.0 \mathrm{~mL})$ and a solution of $t \operatorname{BuLi}(0.50 \mathrm{M}$ in hexane, $0.50 \mathrm{mmol}, 2.5$ equiv) were prepared. The precooled solutions were mixed with an overall $10 \mathrm{~mL} / \mathrm{min}$ flowrate in a T-mixer. The combined stream passed a $0.02 \mathrm{~mL}$ reactor tube $\left(0.1 \mathrm{~s},-20^{\circ} \mathrm{C}\right)$ and was subsequently injected in an empty flask. Stirring was continued for $10 \mathrm{~min}$ at $25^{\circ} \mathrm{C}$ before sat. aq. $\mathrm{NH}_{4} \mathrm{Cl}$ solution was added to quench the reaction mixture. The aqueous phase was extracted three times with EtOAc $(3 \times 30 \mathrm{~mL})$ and the combined organic phases were dried over anhydrous $\mathrm{Na}_{2} \mathrm{SO}_{4}$ and filtrated. After removal of the solvent, flash chromatographical purification (silica gel, isohexane:EtOAc $=95: 5 \rightarrow$ 9:1) afforded the title compound $\mathbf{4 g}$ as a colorless oil $(27 \mathbf{m g}, 0.14$ mmol, $68 \%$ yield).

${ }^{1}$ H-NMR (400 MHz, CDCl3): $\delta$ / ppm = 7.46 - 7.40 (m, 2H), 7.37 - 7.31 (m, 2H), 7.26 - 7.23 $(\mathrm{m}, 1 \mathrm{H}), 7.12-7.06(\mathrm{~m}, 2 \mathrm{H}), 6.96-6.92(\mathrm{~m}, 2 \mathrm{H}), 3.14-2.95(\mathrm{~m}, 2 \mathrm{H}), 2.86(\mathrm{p}, J=6.9 \mathrm{~Hz}$, $1 \mathrm{H}), 1.87(\mathrm{~s}, 1 \mathrm{H}), 1.56(\mathrm{~s}, 3 \mathrm{H}), 1.22(\mathrm{~d}, J=6.9 \mathrm{~Hz}, 6 \mathrm{H})$.

${ }^{13}$ C-NMR (100 MHz, CDCl3): $\delta$ / ppm = 147.9, 147.4, 134.0, 130.7 (2C), 128.2 (2C), 126.7, 126.4 (2C), 125.1 (2C), 74.5, 50.1, 33.8, 29.6, 24.1, 24.1.

IR (Diamond-ATR, neat): $\tilde{v} / \mathrm{cm}^{-1}=3448,3057,3025,2960,2870,1514,1494,1456,1446$, 1420, 1373, 1363, 1284, 1263, 1218, 1177, 1142, 1100, 1065, 1050, 1028, 1021, 940, 910, 866, $843,811,764,747,721,698$.

MS (EI, 70 eV): $m / z(\%)=134$ (61), 121 (80), 119 (100), 117 (24), 115 (14), 91 (20), 43 (39).

HRMS (EI-orbitrap): $m / z$ : [M] calcd for $\mathrm{C}_{18} \mathrm{H}_{22} \mathrm{O} 236.1565$; Found 236.1559. 


\section{2-(3-((Tert-butyldimethylsilyl)oxy)phenyl)-1-phenylethan-1-ol (7a)}<smiles>Oc1cccc(CC(O)c2ccccc2)c1</smiles>

According to the TP1, a solution of tert-butyl(3-(iodomethyl)phenoxy)dimethylsilane (5a, $0.20 \mathrm{M}, 0.20 \mathrm{mmol}, 1.0$ equiv) and benzaldehyde (2a, $0.30 \mathrm{M}, 0.30 \mathrm{mmol}, 1.5$ equiv) in THF (total volume: $1.0 \mathrm{~mL}$ ) and a solution of $t \mathrm{BuLi}(0.50 \mathrm{M}$ in hexane, $0.50 \mathrm{mmol}, 2.5$ equiv) were prepared. The precooled solutions were mixed with an overall $10 \mathrm{~mL} / \mathrm{min}$ flowrate in a T-mixer. The combined stream passed a $0.02 \mathrm{~mL}$ reactor tube $\left(0.1 \mathrm{~s},-40^{\circ} \mathrm{C}\right)$ and was subsequently injected in an empty flask. Stirring was continued for 10 min at $25{ }^{\circ} \mathrm{C}$ before sat. $a q . \mathrm{NH}_{4} \mathrm{Cl}$ solution was added to quench the reaction mixture. The aqueous phase was extracted three times with EtOAc $(3 \times 30 \mathrm{~mL})$ and the combined organic phases were dried over anhydrous $\mathrm{Na}_{2} \mathrm{SO}_{4}$ and filtrated. After removal of the solvent, flash chromatographical purification (silica gel, isohexane:EtOAc $=95: 5 \rightarrow 9: 1)$ afforded the title compound $7 \mathbf{a}$ as a colorless oil $(27 \mathrm{mg}, 0.10$ mmol, 50\% yield).

${ }^{1}$ H-NMR (400 MHz, CDCl3): $\delta /$ ppm = $7.26-7.23(\mathrm{~m}, 3 \mathrm{H}), 7.21-7.17(\mathrm{~m}, 1 \mathrm{H}), 7.06(\mathrm{t}, J=$ $7.8 \mathrm{~Hz}, 1 \mathrm{H}), 6.70(\mathrm{dt}, J=7.6,1.3 \mathrm{~Hz}, 1 \mathrm{H}), 6.62(\mathrm{ddd}, J=8.1,2.5,1.1 \mathrm{~Hz}, 1 \mathrm{H}), 6.57(\mathrm{t}, J=2.1$ $\mathrm{Hz}, 1 \mathrm{H}), 4.78(\mathrm{dd}, J=8.3,5.1 \mathrm{~Hz}, 1 \mathrm{H}), 2.99-2.69(\mathrm{~m}, 2 \mathrm{H}), 1.17-1.14(\mathrm{~m}, 1 \mathrm{H}), 0.88(\mathrm{~s}, 9 \mathrm{H})$, $0.07(\mathrm{~s}, 6 \mathrm{H})$.

${ }^{13}$ C-NMR (100 MHz, CDCl $): \delta$ / ppm = 155.9, 143.8, 139.6, 129.6, 128.5 (2C), 127.7, 126.0 (2C), 122.6, 121.4, 118.5, 75.4, 46.1, 25.8 (3C), 18.3, -4.3 (2C).

IR (Diamond-ATR, neat): $\tilde{v} / \mathrm{cm}^{-1}=3031,2955,2929,2857,1602,1584,1485,1472,1463$, 1442, 1390, 1362, 1272, 1252, 1158, 1043, 1028, 1004, 977, 939, 887, 872, 837, 779, 755, 696, 665 .

MS (EI, 70 eV): $m / z(\%)=310$ (20), 269 (12), 253 (66), 222 (100), 178 (10), 165 (54), 149 (10), 107 (13), 75 (10).

HRMS (EI-orbitrap): $m / z:[\mathrm{M}-\mathrm{OH}]^{+}$calcd for $\mathrm{C}_{20} \mathrm{H}_{27} \mathrm{OSi}^{+}$311.1826; Found 311.1829. 


\section{2-(3-((Tert-butyldimethylsilyl)oxy)phenyl)-1-cyclohexylethan-1-ol (7b)}<smiles>COc1cccc(CC(O)C2CCCCC2)c1</smiles>

According to the TP1, a solution of tert-butyl(3-(iodomethyl)phenoxy)dimethylsilane (5a, $0.20 \mathrm{M}, 0.20 \mathrm{mmol}, 1.0$ equiv) and cyclohexane carboxaldehyde (2d, $0.30 \mathrm{M}, 0.30 \mathrm{mmol}, 1.5$ equiv) in THF (total volume: $1.0 \mathrm{~mL})$ and a solution of $t \mathrm{BuLi}(0.50 \mathrm{M}$ in hexane, $0.50 \mathrm{mmol}$, 2.5 equiv) were prepared. The precooled solutions were mixed with an overall $10 \mathrm{~mL} / \mathrm{min}$ flowrate in a T-mixer. The combined stream passed a $0.02 \mathrm{~mL}$ reactor tube $\left(0.1 \mathrm{~s},-78{ }^{\circ} \mathrm{C}\right)$ and was subsequently injected in an empty flask. Stirring was continued for $10 \mathrm{~min}$ at $25^{\circ} \mathrm{C}$ before sat. $a q . \mathrm{NH}_{4} \mathrm{Cl}$ solution was added to quench the reaction mixture. The aqueous phase was extracted three times with EtOAc $(3 \times 30 \mathrm{~mL})$ and the combined organic phases were dried over anhydrous $\mathrm{Na}_{2} \mathrm{SO}_{4}$ and filtrated. After removal of the solvent, flash chromatographical purification (silica gel, isohexane:EtOAc $=95: 5 \rightarrow 9: 1$ ) afforded the title compound $7 \mathbf{b}$ as a colorless oil ( $35 \mathrm{mg}, 0.12 \mathrm{mmol}, 62 \%$ yield).

${ }^{1}$ H-NMR (400 MHz, CDCl3): $\delta / \mathrm{ppm}=7.19-7.13(\mathrm{~m}, 1 \mathrm{H}), 6.81(\mathrm{dt}, J=7.6,1.4 \mathrm{~Hz}, 1 \mathrm{H})$, $6.71(\mathrm{ddt}, J=6.8,3.2,1.7 \mathrm{~Hz}, 2 \mathrm{H}), 3.55(\mathrm{ddd}, J=9.2,5.5,3.5 \mathrm{~Hz}, 1 \mathrm{H}), 2.83(\mathrm{dd}, J=13.5,3.5$ $\mathrm{Hz}, 1 \mathrm{H}), 2.54(\mathrm{dd}, J=13.5,9.4 \mathrm{~Hz}, 1 \mathrm{H}), 1.95-1.87(\mathrm{~m}, 1 \mathrm{H}), 1.83-1.66(\mathrm{~m}, 4 \mathrm{H}), 1.41(\mathrm{dtt}, J$ = 11.6, 6.1, 3.0 Hz, 1H), $1.31-1.11(\mathrm{~m}, 6 \mathrm{H}), 0.98(\mathrm{~s}, 9 \mathrm{H}), 0.19(\mathrm{~s}, 6 \mathrm{H})$.

${ }^{13}$ C-NMR (100 MHz, $\mathbf{C D C l}$ ): $\delta$ / ppm = 155.9, 140.8, 129.6, 122.5, 121.3, 118.2, 77.0, 43.2, 40.9, 29.5, 28.1, 26.7, 26.5, 26.3, 25.8 (3C), 18.3, -4.2 (2C).

IR (Diamond-ATR, neat): $\tilde{v} / \mathrm{cm}^{-1}=2927,2854,1602,1584,1485,1472,1463,1444,1390$, 1362, 1306, 1276, 1252, 1158, 1099, 1085, 1034, 1004, 978, 955, 891, 837, 779, 717, 696, 665. MS (EI, 70 eV): m/z (\%) = 223 (12), 222 (100), 183 (24), 181 (99), 177 (14), 166 (10), 165 (57), 164 (10), 163 (14), 149 (13), 75 (11).

HRMS (EI-orbitrap): m/z: [M] calcd for $\mathrm{C}_{20} \mathrm{H}_{34} \mathrm{O}_{2} \mathrm{Si}$ 334.2328; Found 334.2320. 


\section{1-Cyclohexyl-2-(4-(methylthio)phenyl)ethan-1-ol (7c)}<smiles>CSc1ccc(CC(O)C2CCCCC2)cc1</smiles>

According to the TP1, a solution of (4-(iodomethyl)phenyl)(methyl)sulfane $(\mathbf{5 b}, 0.20 \mathrm{M}$, $0.20 \mathrm{mmol}, 1.0$ equiv) and cyclohexanecarboxaldehyde (2d, $0.30 \mathrm{M}, 0.30 \mathrm{mmol}, 1.5$ equiv) in THF (total volume: $1.0 \mathrm{~mL}$ ) and a solution of $t \mathrm{BuLi}(0.50 \mathrm{M}$ in hexane, $0.50 \mathrm{mmol}, 2.5$ equiv) were prepared. The precooled solutions were mixed with an overall $10 \mathrm{~mL} / \mathrm{min}$ flowrate in a Tmixer. The combined stream passed a $0.02 \mathrm{~mL}$ reactor tube $\left(0.1 \mathrm{~s},-30^{\circ} \mathrm{C}\right)$ and was subsequently injected in an empty flask. Stirring was continued for 10 min at $25{ }^{\circ} \mathrm{C}$ before sat. aq. $\mathrm{NH}_{4} \mathrm{Cl}$ solution was added to quench the reaction mixture. The aqueous phase was extracted three times with EtOAc $(3 \times 30 \mathrm{~mL})$ and the combined organic phases were dried over anhydrous $\mathrm{Na}_{2} \mathrm{SO}_{4}$ and filtrated. After removal of the solvent, flash chromatographical purification (silica gel, isohexane:EtOAc = 95:5) afforded the title compound 7c as a slightly yellow oil (34 $\mathrm{mg}, 0.14$ mmol, $68 \%$ yield).

${ }^{1}$ H-NMR (400 MHz, CDCl3): $\delta$ / ppm = $7.24-7.19(\mathrm{~m}, 2 \mathrm{H}), 7.15(\mathrm{~d}, J=8.3 \mathrm{~Hz}, 2 \mathrm{H}), 3.54$ (s, 1H), $2.83(\mathrm{dd}, J=13.7,3.4 \mathrm{~Hz}, 1 \mathrm{H}), 2.56(\mathrm{dd}, J=13.7,9.4 \mathrm{~Hz}, 1 \mathrm{H}), 2.47$ (s, 3H), 1.90 (d, $J=$ $12.5 \mathrm{~Hz}, 1 \mathrm{H}), 1.83-1.63(\mathrm{~m}, 4 \mathrm{H}), 1.48-1.33(\mathrm{~m}, 2 \mathrm{H}), 1.33-1.00(\mathrm{~m}, 5 \mathrm{H})$.

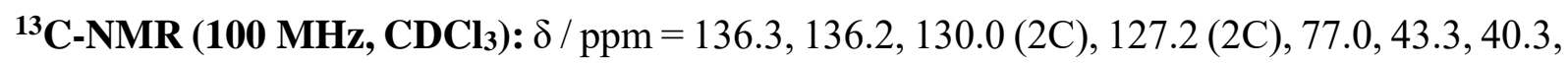
29.5, 28.1, 26.7, 26.4, 26.7, 16.3 .

IR (Diamond-ATR, neat): $\tilde{v} / \mathrm{cm}^{-1}=3331,3259,2930,2917,2882,2848,1492,1446,1435$, 1424, 1404, 1108, 1098, 1083, 1059, 1038, 1018, 1010, 972, 956, 890, 844, 835, 802, 662.

MS (EI, 70 eV): $m / z(\%)=138$ (100), 137 (28), 123 (99), 122 (11), 91 (30).

HRMS (EI-orbitrap): $m / z$ : [M] calcd for $\mathrm{C}_{15} \mathrm{H}_{22} \mathrm{OS} 250.1391$; Found 250.1383. 


\section{1-(3-Methoxyphenyl)-2-(4-methoxyphenyl)ethan-1-ol (7d)}<smiles>COc1ccc(CC(O)c2cccc(OC)c2)cc1</smiles>

According to the TP1, a solution of 1-(iodomethyl)-4-methoxybenzene (5c, $0.20 \mathrm{M}, 0.20 \mathrm{mmol}$, 1.0 equiv) and $m$-anisaldehyde $(\mathbf{2 b}, 0.30 \mathrm{M}, 0.30 \mathrm{mmol}, 1.5$ equiv) in THF (total volume: $1.0 \mathrm{~mL})$ and a solution of $t \mathrm{BuLi}(0.50 \mathrm{M}$ in hexane, $0.50 \mathrm{mmol}, 2.5$ equiv) were prepared. The precooled solutions were mixed with an overall $10 \mathrm{~mL} / \mathrm{min}$ flowrate in a T-mixer. The combined stream passed a $0.02 \mathrm{~mL}$ reactor tube $\left(0.1 \mathrm{~s},-20^{\circ} \mathrm{C}\right)$ and was subsequently injected in an empty flask. Stirring was continued for $10 \mathrm{~min}$ at $25^{\circ} \mathrm{C}$ before sat. aq. $\mathrm{NH}_{4} \mathrm{Cl}$ solution was added to quench the reaction mixture. The aqueous phase was extracted three times with EtOAc $(3 \times 30 \mathrm{~mL})$ and the combined organic phases were dried over anhydrous $\mathrm{Na}_{2} \mathrm{SO}_{4}$ and filtrated. After removal of the solvent, flash chromatographical purification (silica gel, isohexane:EtOAc $=95: 5 \rightarrow$ 9:1) afforded the title compound $\mathbf{7 d}$ as a colorless oil $(35 \mathrm{mg}, 0.13$ mmol, $67 \%$ yield).

${ }^{1}$ H-NMR (400 MHz, CDCl3): $\delta$ / ppm = 7.33 - 7.24 (m, 1H), 7.19 - 7.10 (m, 2H), 6.98 - 6.91 $(\mathrm{m}, 2 \mathrm{H}), 6.91-6.81(\mathrm{~m}, 3 \mathrm{H}), 4.85(\mathrm{ddd}, J=8.7,4.6,2.0 \mathrm{~Hz}, 1 \mathrm{H}), 3.83(\mathrm{~s}, 3 \mathrm{H}), 3.81$ (s, 3H), $3.06-2.87(\mathrm{~m}, 2 \mathrm{H}), 1.98(\mathrm{~d}, J=2.7 \mathrm{~Hz}, 1 \mathrm{H})$.

${ }^{13}$ C-NMR (100 MHz, CDCl $): \delta /$ ppm = 159.8, 158.5, 145.7, 130.6 (2C), 130.0, 129.5, 118.4, $114.1(2 \mathrm{C}), 113.3,111.4,75.5,55.4,55.4,45.3$.

IR (Diamond-ATR, neat): $\tilde{v} / \mathrm{cm}^{-1}=3422,3000,2935,2835,1610,1602,1585,1511,1488$, $1464,1455,1435,1318,1300,1285,1242,1177,1149,1108,1033,876,863,846,817,784$, $773,730,715,697$.

MS (EI, $70 \mathrm{eV}): m / z(\%)=122(100), 121(45), 109$ (25), 94 (10).

HRMS (EI-orbitrap): $m / z$ : [M - $\left.\mathrm{H}_{2} \mathrm{O}\right]$ calcd for $\mathrm{C}_{16} \mathrm{H}_{16} \mathrm{O}_{2} 240.1150$; Found 240.1144. 


\section{1-(2,4-Dimethylphenyl)-2-(3-methoxyphenyl)ethan-1-ol (7e)}<smiles>COc1cccc(CC(O)c2ccc(C)cc2C)c1</smiles>

According to the TP1, a solution of 1-(iodomethyl)-3-methoxybenzene (5d, 0.20 M, 0.20 mmol, 1.0 equiv) and 2,4-dimethylbenzaldehyde (2f, $0.30 \mathrm{M}, 0.30 \mathrm{mmol}, 1.5$ equiv) in THF (total volume: $1.0 \mathrm{~mL})$ and a solution of $t \mathrm{BuLi}(0.50 \mathrm{M}$ in hexane, $0.50 \mathrm{mmol}, 2.5$ equiv) were prepared. The precooled solutions were mixed with an overall $10 \mathrm{~mL} / \mathrm{min}$ flowrate in a T-mixer. The combined stream passed a $0.02 \mathrm{~mL}$ reactor tube $\left(0.1 \mathrm{~s},-30^{\circ} \mathrm{C}\right)$ and was subsequently injected in an empty flask. Stirring was continued for $10 \mathrm{~min}$ at $25^{\circ} \mathrm{C}$ before sat. aq. $\mathrm{NH}_{4} \mathrm{Cl}$ solution was added to quench the reaction mixture. The aqueous phase was extracted three times with EtOAc $(3 \times 30 \mathrm{~mL})$ and the combined organic phases were dried over anhydrous $\mathrm{Na}_{2} \mathrm{SO}_{4}$ and filtrated. After removal of the solvent, flash chromatographical purification (silica gel, isohexane:EtOAc $=95: 5 \rightarrow 9: 1)$ afforded the title compound $7 \mathbf{e}$ as a slightly yellow oil $(27 \mathrm{mg}$, $0.11 \mathrm{mmol}, 53 \%$ yield).

${ }^{1}$ H-NMR (400 MHz, CDCl3): $\delta / \mathrm{ppm}=7.49(\mathrm{~d}, J=7.9 \mathrm{~Hz}, 1 \mathrm{H}), 7.28(\mathrm{~s}, 1 \mathrm{H}), 7.15-7.07$ (m, 1H), $7.04-6.98(\mathrm{~m}, 1 \mathrm{H}), 6.91-6.78(\mathrm{~m}, 3 \mathrm{H}), 5.13(\mathrm{dd}, J=9.0,4.2 \mathrm{~Hz}, 1 \mathrm{H}), 3.82(\mathrm{~s}, 3 \mathrm{H}), 3.07$ $-2.84(\mathrm{~m}, 2 \mathrm{H}), 2.36(\mathrm{~s}, 3 \mathrm{H}), 2.30(\mathrm{~s}, 3 \mathrm{H}), 1.92(\mathrm{~d}, J=2.2 \mathrm{~Hz}, 1 \mathrm{H})$.

${ }^{13}$ C-NMR (100 MHz, $\left.\mathbf{C D C l}_{3}\right): \delta / \mathrm{ppm}=159.8,140.2$, 139.2, 137.0, 134.4, 131.2, 129.7, 127.1, 125.3, 121.9, 115.2, 112.1, 71.7, 55.3, 45.2, 21.1, 19.1 .

IR (Diamond-ATR, neat): $\tilde{v} / \mathrm{cm}^{-1}=3414,2921,2835,1602,1584,1488,1465,1453,1436$, $1378,1313,1296,1256,1191,1166,1152,1040,996,954,873,856,822,775,750,743,718$, 694.

MS (EI, 70 eV): $m / z(\%)=135$ (88), 123 (10), 122 (100), 121 (13), 107 (70), 105 (22), 91 (57), 79 (12), 78 (11), 77 (15), 65 (11).

HRMS (EI-orbitrap): $m / z:$ : $\left.\mathrm{M}-\mathrm{H}_{2} \mathrm{O}\right]$ calcd for $\mathrm{C}_{17} \mathrm{H}_{18} \mathrm{O} 238.1358$; Found 238.1351. 


\section{2-(3-Methoxybenzyl)bicyclo[2.2.1]heptan-2-ol (7f)}<smiles>COc1cccc(CC2(O)CC3CCC2C3)c1</smiles>

According to the TP1, a solution of 1-(iodomethyl)-3-methoxybenzene (5d, $0.20 \mathrm{M}, 0.20 \mathrm{mmol}$, 1.0 equiv) and norcamphor ( $2 \mathrm{~g}, 0.30 \mathrm{M}, 0.30 \mathrm{mmol}, 1.5$ equiv) in THF (total volume: $1.0 \mathrm{~mL}$ ) and a solution of $t \mathrm{BuLi}(0.50 \mathrm{M}$ in hexane, $0.50 \mathrm{mmol}, 2.5$ equiv $)$ were prepared. The precooled solutions were mixed with an overall $10 \mathrm{~mL} / \mathrm{min}$ flowrate in a T-mixer. The combined stream passed a $0.02 \mathrm{~mL}$ reactor tube $\left(0.1 \mathrm{~s},-30^{\circ} \mathrm{C}\right)$ and was subsequently injected in an empty flask. Stirring was continued for $10 \mathrm{~min}$ at $25{ }^{\circ} \mathrm{C}$ before sat. aq. $\mathrm{NH}_{4} \mathrm{Cl}$ solution was added to quench the reaction mixture. The aqueous phase was extracted three times with EtOAc $(3 \times 30 \mathrm{~mL})$ and the combined organic phases were dried over anhydrous $\mathrm{Na}_{2} \mathrm{SO}_{4}$ and filtrated. After removal of the solvent, flash chromatographical purification (silica gel, isohexane:EtOAc $=95: 5 \rightarrow$ 9:1) afforded the title compound $\mathbf{7 f}$ as a colorless oil (27 $\mathrm{mg}, 0.12 \mathrm{mmol}, 59 \%$ yield, d.r. > 99:1).

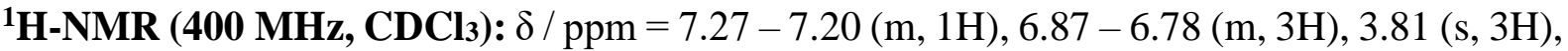
$2.89-2.71(\mathrm{~m}, 2 \mathrm{H}), 2.29-2.22(\mathrm{~m}, 1 \mathrm{H}), 2.19-2.11(\mathrm{~m}, 1 \mathrm{H}), 1.94-1.82(\mathrm{~m}, 1 \mathrm{H}), 1.73$ (ddd, $J=12.9,4.6,2.9 \mathrm{~Hz}, 1 \mathrm{H}), 1.70-1.45(\mathrm{~m}, 3 \mathrm{H}), 1.40-1.22(\mathrm{~m}, 3 \mathrm{H}), 1.12(\mathrm{dd}, J=12.9,3.3 \mathrm{~Hz}$, $1 \mathrm{H})$.

${ }^{13} \mathbf{C}-N M R\left(100 ~ M H z, \mathbf{C D C l}_{3}\right): \delta$ / ppm = 159.6, 139.4, 129.4, 123.1, 116.4, 111.9, 79.3, 55.3, $48.0,45.9,45.8,38.7,37.5,28.8,22.2$.

IR (Diamond-ATR, neat): $\tilde{v} / \mathrm{cm}^{-1}=3392,2935,2838,1612,1586,1512,1487,1462,1446$, 1302, 1243, 1174, 1140, 1110, 1076, 1032, 1006, 960, 948, 870, 831, 811, 786, 772, 755, 740, 688.

MS (EI, 70 eV): $m / z(\%)=122$ (100), 121 (16), 111 (19), 93 (13), 91 (22).

HRMS (EI-orbitrap): m/z: [M] calcd for $\mathrm{C}_{15} \mathrm{H}_{20} \mathrm{O}_{2}$ 232.1463; Found 232.1353. 


\section{2-(3-Methoxybenzyl)adamantan-2-ol (7g)}<smiles>COc1cccc(CC2(O)C3CC4CC(C3)CC2C4)c1</smiles>

According to the TP1, a solution of 1-(iodomethyl)-3-methoxybenzene (5d, $0.20 \mathrm{M}, 0.20 \mathrm{mmol}$, 1.0 equiv) and adamantanone ( $\mathbf{2 h}, 0.30 \mathrm{M}, 0.30 \mathrm{mmol}, 1.5$ equiv) in THF (total volume: $1.0 \mathrm{~mL}$ ) and a solution of $t \mathrm{BuLi}(0.50 \mathrm{M}$ in hexane, $0.50 \mathrm{mmol}, 2.5$ equiv) were prepared. The precooled solutions were mixed with an overall $10 \mathrm{~mL} / \mathrm{min}$ flowrate in a T-mixer. The combined stream passed a $0.02 \mathrm{~mL}$ reactor tube $\left(0.1 \mathrm{~s},-78^{\circ} \mathrm{C}\right)$ and was subsequently injected in an empty flask. Stirring was continued for $10 \mathrm{~min}$ at $25^{\circ} \mathrm{C}$ before sat. aq. $\mathrm{NH}_{4} \mathrm{Cl}$ solution was added to quench the reaction mixture. The aqueous phase was extracted three times with EtOAc $(3 \times 30 \mathrm{~mL})$ and the combined organic phases were dried over anhydrous $\mathrm{Na}_{2} \mathrm{SO}_{4}$ and filtrated. After removal of the solvent, flash chromatographical purification (silica gel, isohexane:EtOAc $=95: 5 \rightarrow 9: 1$ ) afforded the title compound $\mathbf{7 g}$ as a white amorphous solid (46 $\mathrm{mg}, 0.17 \mathrm{mmol}, 85 \%$ yield).

${ }^{1}$ H-NMR (400 MHz, CDCl3): $\delta$ / ppm = 7.25 - 7.19 (m, 1H), $6.86-6.76$ (m, 3H), 3.80 (s, 3H), $2.97(\mathrm{~s}, 2 \mathrm{H}), 2.17(\mathrm{dd}, J=12.6,2.9 \mathrm{~Hz}, 2 \mathrm{H}), 2.14-2.04(\mathrm{~m}, 2 \mathrm{H}), 1.92(\mathrm{p}, J=3.0 \mathrm{~Hz}, 1 \mathrm{H}), 1.84$ - 1.75 (m, 3H), 1.69 (dd, $J=8.3,3.8 \mathrm{~Hz}, 4 \mathrm{H}), 1.57-1.48$ (m, 2H), 1.45 (s, 1H).

${ }^{13}$ C-NMR (100 MHz, CDCl3): $\delta$ / ppm = 159.6, 139.0, 129.3, 123.1, 116.4, 111.9, 74.7, 55.3, 44.0, 38.5, $37.0(2 \mathrm{C}), 34.7$ (2C), 33.1 (2C), 27.6, 27.5.

IR (Diamond-ATR, neat): $\tilde{v} / \mathrm{cm}^{-1}=3510,2962,2942,2930,2899,2853,1742,1600,1590$, 1489, 1471, 1462, 1432, 1374, 1260, 1228, 1168, 1123, 1101, 1084, 1061, 1035, 1010, 993, 929, 922, 791, 739, 716, 694.

MS (EI, 70 eV): $m / z(\%)=255$ (16), 254 (78), 151 (51), 122 (52), 121 (15), 91 (15), 88 (13), 73 (11), 70 (14), 61 (27), 45 (15), 43 (100).

HRMS (EI-orbitrap): m/z: [M - $\mathrm{H}_{2} \mathrm{O}$ ] calcd for $\mathrm{C}_{18} \mathrm{H}_{22} \mathrm{O}$ 254.1671; Found 254.1668. m.p. $\left({ }^{\circ} \mathbf{C}\right): 100.7-104.2$. 


\section{2-(4-(Methylthio)benzyl)adamantan-2-ol (7h)}

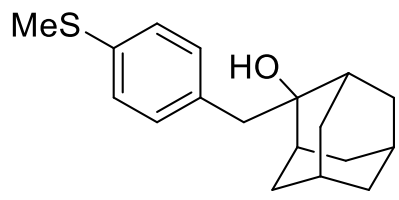

According to the TP1, a solution of (4-(iodomethyl)phenyl)(methyl)sulfane $(\mathbf{5 b}, 0.20 \mathrm{M}$, $0.20 \mathrm{mmol}, 1.0$ equiv) and adamantanone (2h, $0.30 \mathrm{M}, 0.30 \mathrm{mmol}, 1.5$ equiv) in THF (total volume: $1.0 \mathrm{~mL})$ and a solution of $t \mathrm{BuLi}(0.50 \mathrm{M}$ in hexane, $0.50 \mathrm{mmol}, 2.5$ equiv) were prepared. The precooled solutions were mixed with an overall $10 \mathrm{~mL} / \mathrm{min}$ flowrate in a T-mixer. The combined stream passed a $0.02 \mathrm{~mL}$ reactor tube $\left(0.1 \mathrm{~s},-30^{\circ} \mathrm{C}\right)$ and was subsequently injected in an empty flask. Stirring was continued for $10 \mathrm{~min}$ at $25^{\circ} \mathrm{C}$ before sat. aq. $\mathrm{NH}_{4} \mathrm{Cl}$ solution was added to quench the reaction mixture. The aqueous phase was extracted three times with EtOAc $(3 \times 30 \mathrm{~mL})$ and the combined organic phases were dried over anhydrous $\mathrm{Na}_{2} \mathrm{SO}_{4}$ and filtrated. After removal of the solvent, flash chromatographical purification (silica gel, isohexane:EtOAc $=95: 5)$ afforded the title compound $7 \mathbf{h}$ as a slightly yellow oil (44 $\mathrm{mg}, 0.15$ mmol, $76 \%$ yield).

${ }^{1}$ H-NMR (400 MHz, CDCl3): $\delta / \mathrm{ppm}=7.23-7.13(\mathrm{~m}, 4 \mathrm{H}), 2.95(\mathrm{~s}, 2 \mathrm{H}), 2.47(\mathrm{~s}, 3 \mathrm{H}), 2.19$ $-2.12(\mathrm{~m}, 2 \mathrm{H}), 2.12-2.03(\mathrm{~m}, 2 \mathrm{H}), 1.91$ (p, $J=3.1 \mathrm{~Hz}, 1 \mathrm{H}), 1.83-1.74(\mathrm{~m}, 3 \mathrm{H}), 1.68$ (dt, $J$ $=18.6,3.3 \mathrm{~Hz}, 4 \mathrm{H}), 1.52(\mathrm{dq}, J=12.6,1.9 \mathrm{~Hz}, 2 \mathrm{H}), 1.36(\mathrm{~s}, 1 \mathrm{H})$.

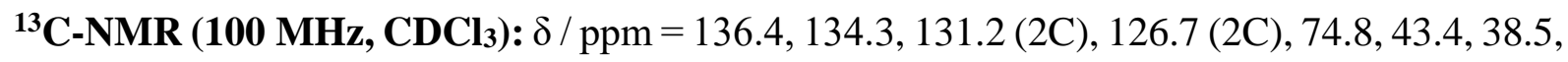
36.9 (2C), 34.7 (2C), $33.1(2 \mathrm{C}), 27.6,27.4,16.1$.

IR (Diamond-ATR, neat): $\tilde{v} / \mathrm{cm}^{-1}=3422,3393,2943,2937,2911,2904,2893,2849,1495$, 1452, 1439, 1402, 1354, 1351, 1287, 1208, 1200, 1196, 1160, 1122, 1096, 1068, 1056, 1042, $1019,1005,994,964,952,925,894,848,814,804,733,661$.

MS (EI, 70 eV): $m / z(\%)=151$ (48), 138 (100), 137 (10), 91 (16).

HRMS (EI-orbitrap): $m / z$ : [M - $\mathrm{H}_{2} \mathrm{O}$ ] calcd for $\mathrm{C}_{18} \mathrm{H}_{22} \mathrm{~S}$ 270.1442; Found 270.1435. 


\section{2-(2-Fluorophenyl)-1-phenylethan-1-ol (10a)}<smiles>OC(Cc1ccccc1F)c1ccccc1</smiles>

According to the TP1, a solution of 1-fluoro-2-(iodomethyl)benzene (8a, $0.20 \mathrm{M}, 0.20 \mathrm{mmol}$, 1.0 equiv) and benzaldehyde (2a, $0.30 \mathrm{M}, 0.30 \mathrm{mmol}, 1.5$ equiv) in THF (total volume: $1.0 \mathrm{~mL}$ ) and a solution of $t \mathrm{BuLi}(0.50 \mathrm{M}$ in hexane, $0.50 \mathrm{mmol}, 2.5$ equiv) were prepared. The precooled solutions were mixed with an overall $10 \mathrm{~mL} / \mathrm{min}$ flowrate in a T-mixer. The combined stream passed a $0.02 \mathrm{~mL}$ reactor tube $\left(0.1 \mathrm{~s},-78^{\circ} \mathrm{C}\right)$ and was subsequently injected in an empty flask. Stirring was continued for $10 \mathrm{~min}$ at $25^{\circ} \mathrm{C}$ before sat. aq. $\mathrm{NH}_{4} \mathrm{Cl}$ solution was added to quench the reaction mixture. The aqueous phase was extracted three times with EtOAc $(3 \times 30 \mathrm{~mL})$ and the combined organic phases were dried over anhydrous $\mathrm{Na}_{2} \mathrm{SO}_{4}$ and filtrated. After removal of the solvent, flash chromatographical purification (silica gel, isohexane:EtOAc $=95: 5 \rightarrow$ 9:1) afforded the title compound $\mathbf{1 0 a}$ as a colorless oil (30 $\mathrm{mg}, 0.14 \mathrm{mmol}, 70 \%$ yield).

${ }^{1}$ H-NMR (400 MHz, CDCl3): $\delta$ / ppm = 7.29 - 7.25 (m, 3H), $7.24-7.13$ (m, 3H), 6.90 - 6.79 $(\mathrm{m}, 3 \mathrm{H}), 4.81(\mathrm{t}, J=6.7 \mathrm{~Hz}, 1 \mathrm{H}), 2.93(\mathrm{~d}, J=6.6 \mathrm{~Hz}, 2 \mathrm{H}), 1.87(\mathrm{~s}, 1 \mathrm{H})$.

${ }^{13}$ C-NMR (100 MHz, CDCl $): \delta / \mathrm{ppm}=163.0(\mathrm{~d}, J=245.7 \mathrm{~Hz}), 143.7,140.7(\mathrm{~d}, J=7.3 \mathrm{~Hz})$, $130.0(\mathrm{~d}, J=8.3 \mathrm{~Hz}), 128.7,128.0(2 \mathrm{C}), 126.0(2 \mathrm{C}), 125.3$ (d, $J=2.8 \mathrm{~Hz}), 116.5$ (d, $J=21.0$ $\mathrm{Hz}), 113.6(\mathrm{~d}, J=21.0 \mathrm{~Hz}), 75.3,45.8$.

IR (Diamond-ATR, neat): $\tilde{v} / \mathrm{cm}^{-1}=3372,1615,1588,1488,1448,1248,1201,1139,1075$, 1041, 1028, 1010, 960, 948, 913, 868, 778, 755, 738, 698, 690.

MS (EI, 70 eV): $m / z(\%)=199$ (12), 198 (60), 197 (45), 196 (29), 183 (21), 177 (12), 105 (47), 77 (19), 70 (14), 61 (24), 45 (16), 44 (42), 43 (100), 42 (10).

HRMS (EI-orbitrap): $m / z$ : [M - $\mathrm{H}_{2} \mathrm{O}$ ] calcd for $\mathrm{C}_{14} \mathrm{H}_{11} \mathrm{~F}$ 198.0845; Found 198.0803. 


\section{2-(2-Fluorophenyl)-1-(4-methoxyphenyl)ethan-1-ol (10b)}<smiles>COc1ccc(C(O)Cc2ccccc2F)cc1</smiles>

According to the TP1, a solution of 1-fluoro-2-(iodomethyl)benzene (8a, 0.20 M, $0.20 \mathrm{mmol}$, 1.0 equiv) and $p$-methoxybenzaldehyde (2c, $0.30 \mathrm{M}, 0.30 \mathrm{mmol}, 1.5$ equiv) in THF (total volume: $1.0 \mathrm{~mL})$ and a solution of $t \operatorname{BuLi}(0.50 \mathrm{M}$ in hexane, $0.50 \mathrm{mmol}, 2.5$ equiv) were prepared. The precooled solutions were mixed with an overall $10 \mathrm{~mL} / \mathrm{min}$ flowrate in a T-mixer. The combined stream passed a $0.02 \mathrm{~mL}$ reactor tube $\left(0.1 \mathrm{~s},-78^{\circ} \mathrm{C}\right)$ and was subsequently injected in an empty flask. Stirring was continued for $10 \mathrm{~min}$ at $25{ }^{\circ} \mathrm{C}$ before sat. aq. $\mathrm{NH}_{4} \mathrm{Cl}$ solution was added to quench the reaction mixture. The aqueous phase was extracted three times with EtOAc $(3 \times 30 \mathrm{~mL})$ and the combined organic phases were dried over anhydrous $\mathrm{Na}_{2} \mathrm{SO}_{4}$ and filtrated. After removal of the solvent, flash chromatographical purification (silica gel, isohexane:EtOAc $=95: 5 \rightarrow$ 9:1) afforded the title compound 10b as a yellow oil (26 mg, 0.11 mmol, 53\% yield).

${ }^{1}$ H-NMR (400 MHz, CDCl3): $\delta / \mathrm{ppm}=7.30-7.19(\mathrm{~m}, 3 \mathrm{H}), 6.95(\mathrm{dt}, J=7.6,1.2 \mathrm{~Hz}, 1 \mathrm{H})$, $6.93-6.84(\mathrm{~m}, 4 \mathrm{H}), 4.85(\mathrm{dd}, J=7.7,5.6 \mathrm{~Hz}, 1 \mathrm{H}), 3.81(\mathrm{~s}, 3 \mathrm{H}), 3.07-2.92(\mathrm{~m}, 2 \mathrm{H}), 1.92$ (s, $1 \mathrm{H})$.

${ }^{13}$ C-NMR (100 MHz, CDCl3): $\delta / \mathrm{ppm}=162.8(\mathrm{~d}, J=245.7 \mathrm{~Hz}), 159.2,140.8(\mathrm{~d}, J=7.3 \mathrm{~Hz})$, 135.7, $129.8(\mathrm{~d}, J=8.4 \mathrm{~Hz}), 127.2(2 \mathrm{C}), 125.2(\mathrm{~d}, J=2.8 \mathrm{~Hz}), 116.4(\mathrm{~d}, J=21.0 \mathrm{~Hz}), 113.9$ (2C), $113.4(\mathrm{~d}, J=21.0 \mathrm{~Hz}), 74.8,55.3,45.6$.

IR (Diamond-ATR, neat): $\tilde{v} / \mathrm{cm}^{-1}=3392,2935,1612,1586,1512,1487,1462,1446,1302$, 1243, 1174, 1140, 1110, 1076, 1032, 1006, 960, 948, 870, 831, 811, 786, 772, 755, 740, 688.

MS (EI, 70 eV): $m / z(\%)=228$ (25), 138 (10), 137 (100), 135 (16), 109 (15), 88 (11), 77 (10), 73 (10), 70 (11), 61 (20), 45 (11), 43 (74).

HRMS (EI-orbitrap): m/z: [M] calcd for $\mathrm{C}_{15} \mathrm{H}_{15} \mathrm{FO}_{2}$ 246.1056; Found 246.1062. 


\section{2-(2-Fluorobenzyl)adamantan-2-ol (10c)}<smiles>OC1(Cc2ccccc2F)C2CC3CC(C2)CC1C3</smiles>

According to the TP1, a solution of 1-fluoro-2-(iodomethyl)benzene (8a, 0.20 M, $0.20 \mathrm{mmol}$, 1.0 equiv) and adamantanone ( $\mathbf{2 h}, 0.30 \mathrm{M}, 0.30 \mathrm{mmol}, 1.5$ equiv) in THF (total volume: $1.0 \mathrm{~mL}$ ) and a solution of $t \mathrm{BuLi}(0.50 \mathrm{M}$ in hexane, $0.50 \mathrm{mmol}, 2.5$ equiv) were prepared. The precooled solutions were mixed with an overall $10 \mathrm{~mL} / \mathrm{min}$ flowrate in a T-mixer. The combined stream passed a $0.02 \mathrm{~mL}$ reactor tube $\left(0.1 \mathrm{~s}, 0{ }^{\circ} \mathrm{C}\right)$ and was subsequently injected in an empty flask. Stirring was continued for $10 \mathrm{~min}$ at $25^{\circ} \mathrm{C}$ before sat. $a q . \mathrm{NH}_{4} \mathrm{Cl}$ solution was added to quench the reaction mixture. The aqueous phase was extracted three times with EtOAc $(3 \times 30 \mathrm{~mL})$ and the combined organic phases were dried over anhydrous $\mathrm{Na}_{2} \mathrm{SO}_{4}$ and filtrated. After removal of the solvent, flash chromatographical purification (silica gel, isohexane:EtOAc $=95: 5 \rightarrow$ 9:1) afforded the title compound 10c as a white amorphous solid (23 mg, $0.09 \mathrm{mmol}, 44 \%$ yield).

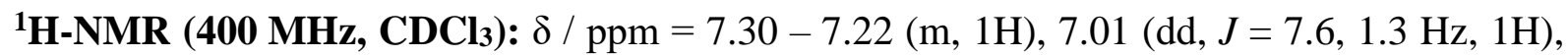
$6.99-6.90(\mathrm{~m}, 2 \mathrm{H}), 2.99(\mathrm{~s}, 2 \mathrm{H}), 2.21-2.11(\mathrm{~m}, 2 \mathrm{H}), 2.04(\mathrm{~d}, J=3.1 \mathrm{~Hz}, 2 \mathrm{H}), 1.92(\mathrm{~s}, 1 \mathrm{H})$, $1.85-1.75(\mathrm{~m}, 3 \mathrm{H}), 1.69(\mathrm{dt}, J=17.8,3.3 \mathrm{~Hz}, 4 \mathrm{H}), 1.54(\mathrm{dt}, J=12.6,1.7 \mathrm{~Hz}, 2 \mathrm{H}), 1.38(\mathrm{~s}$, $1 \mathrm{H})$.

${ }^{13}$ C-NMR (100 MHz, CDCl3): $\delta / \mathrm{ppm}=162.8(\mathrm{~d}, J=245.4 \mathrm{~Hz}), 140.1(\mathrm{~d}, J=7.2 \mathrm{~Hz}), 129.6$ $(\mathrm{d}, J=8.4 \mathrm{~Hz}), 126.4(\mathrm{~d}, J=2.8 \mathrm{~Hz}), 117.6(\mathrm{~d}, J=20.6 \mathrm{~Hz}), 113.5$ (d, $J=21.0 \mathrm{~Hz}), 74.9,43.8$ (d, $J=1.8 \mathrm{~Hz}), 38.5,37.0$ (2C), 34.7 (2C), 33.1 (2C), 27.5, 27.4.

IR (Diamond-ATR, neat): $\tilde{v} / \mathrm{cm}^{-1}=3478,2905,2855,1616,1586,1487,1444,1251,1141$, 1098, 1042, 1018, 1010, 994, 927, 786, 747, 718, 688.

MS (EI, 70 eV): $m / z(\%)=152$ (10), 151 (100), 109 (34), 91 (23), 83 (11), 79 (16).

HRMS (EI-orbitrap): $m / z$ : [M - $\mathrm{H}_{2} \mathrm{O}$ ] calcd for $\mathrm{C}_{17} \mathrm{H}_{19} \mathrm{~F}$ 242.1471; Found 242.1464.

m.p. $\left({ }^{\circ} \mathbf{C}\right): 79.0-81.6$. 


\section{2-(2-Chlorophenyl)-1-phenylethan-1-ol (10d)}<smiles>OC(Cc1ccccc1Cl)c1ccccc1</smiles>

According to the TP1, a solution of 1-chloro-2-(iodomethyl)benzene $(\mathbf{8 b}, 0.20 \mathrm{M}, 0.20 \mathrm{mmol}$, 1.0 equiv) and benzaldehyde (2a, $0.30 \mathrm{M}, 0.30 \mathrm{mmol}, 1.5$ equiv) in THF (total volume: $1.0 \mathrm{~mL}$ ) and a solution of $t \mathrm{BuLi}(0.50 \mathrm{M}$ in hexane, $0.50 \mathrm{mmol}, 2.5$ equiv) were prepared. The precooled solutions were mixed with an overall $10 \mathrm{~mL} / \mathrm{min}$ flowrate in a T-mixer. The combined stream passed a $0.02 \mathrm{~mL}$ reactor tube $\left(0.1 \mathrm{~s},-78^{\circ} \mathrm{C}\right)$ and was subsequently injected in an empty flask. Stirring was continued for $10 \mathrm{~min}$ at $25^{\circ} \mathrm{C}$ before sat. aq. $\mathrm{NH}_{4} \mathrm{Cl}$ solution was added to quench the reaction mixture. The aqueous phase was extracted three times with EtOAc $(3 \times 30 \mathrm{~mL})$ and the combined organic phases were dried over anhydrous $\mathrm{Na}_{2} \mathrm{SO}_{4}$ and filtrated. After removal of the solvent, flash chromatographical purification (silica gel, isohexane:EtOAc $=95: 5 \rightarrow$ 9:1) afforded the title compound $\mathbf{1 0 d}$ as white crystals (37 $\mathrm{mg}, 0.16 \mathrm{mmol}, 80 \%$ yield).

${ }^{1}$ H-NMR (400 MHz, CDCl3): $\delta$ / ppm = 7.35 - 7.25 (m, 5H), 7.25 - 7.19 (m, 1H), $7.14-7.07$ (m, 3H), 4.95 (ddd, $J=8.8,4.3,2.7 \mathrm{~Hz}, 1 \mathrm{H}), 3.14$ (dd, $J=13.7,4.4 \mathrm{~Hz}, 1 \mathrm{H}), 3.00$ (dd, $J=13.7$, $8.9 \mathrm{~Hz}, 1 \mathrm{H}), 1.89(\mathrm{~d}, J=3.1 \mathrm{~Hz}, 1 \mathrm{H})$.

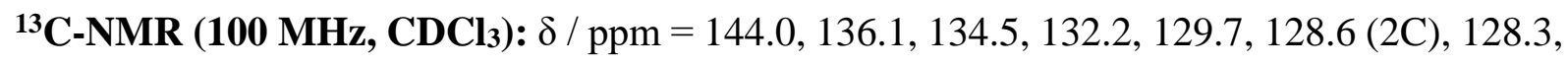
$127.8,126.8,125.9(2 \mathrm{C}), 73.6,44.0$.

IR (Diamond-ATR, neat): $\tilde{v} / \mathrm{cm}^{-1}=3288,3260,3234,3192,3061,3030,2927,1472,1444$, 1418, 1400, 1326, 1288, 1203, 1049, 1040, 1030, 1002, 994, 910, 874, 747, 695, 682.

MS (EI, 70 eV): m/z (\%) = 128 (34), 126 (100), 125 (13), 107 (93), 91 (25), 89 (11), 79 (80), 77 (19).

HRMS (EI-orbitrap): $m / z:[\mathrm{M}-\mathrm{OH}]^{+}$calcd for $\mathrm{C}_{14} \mathrm{H}_{12} \mathrm{Cl}^{+}$215.0622; Found 215.0619. m.p. $\left({ }^{\circ} \mathbf{C}\right): 72.3-73.1$. 


\section{1-Phenyl-2-(3-(trifluoromethyl)phenyl)ethan-1-ol (10e)}<smiles>OC(Cc1cccc(C(F)(F)F)c1)c1ccccc1</smiles>

According to the TP1, a solution of 1-(iodomethyl)-3-(trifluoromethyl)benzene $(\mathbf{8 c}, 0.20 \mathrm{M}$, $0.20 \mathrm{mmol}, 1.0$ equiv) and benzaldehyde $(\mathbf{2 a}, 0.30 \mathrm{M}, 0.30 \mathrm{mmol}, 1.5$ equiv) in THF (total volume: $1.0 \mathrm{~mL})$ and a solution of $t \mathrm{BuLi}(0.50 \mathrm{M}$ in hexane, $0.50 \mathrm{mmol}, 2.5$ equiv) were prepared. The precooled solutions were mixed with an overall $10 \mathrm{~mL} / \mathrm{min}$ flowrate in a T-mixer. The combined stream passed a $0.02 \mathrm{~mL}$ reactor tube $\left(0.1 \mathrm{~s},-78^{\circ} \mathrm{C}\right)$ and was subsequently injected in an empty flask. Stirring was continued for 10 min at $25{ }^{\circ} \mathrm{C}$ before sat. $a q . \mathrm{NH}_{4} \mathrm{Cl}$ solution was added to quench the reaction mixture. The aqueous phase was extracted three times with EtOAc $(3 \times 30 \mathrm{~mL})$ and the combined organic phases were dried over anhydrous $\mathrm{Na}_{2} \mathrm{SO}_{4}$ and filtrated. After removal of the solvent, flash chromatographical purification (silica gel, isohexane:EtOAc $=95: 5 \rightarrow 9: 1)$ afforded the title compound $\mathbf{1 0 e}$ as a colorless oil $(34 \mathrm{mg}, 0.13$ mmol, $64 \%$ yield).

${ }^{1}$ H-NMR (400 MHz, CDCl3): $\delta$ / ppm = $7.45-7.38(\mathrm{~m}, 1 \mathrm{H}), 7.37-7.32(\mathrm{~m}, 2 \mathrm{H}), 7.32-7.20$ $(\mathrm{m}, 6 \mathrm{H}), 4.90-4.72(\mathrm{~m}, 1 \mathrm{H}), 3.10-2.88(\mathrm{~m}, 2 \mathrm{H}), 1.86(\mathrm{~d}, J=2.8 \mathrm{~Hz}, 1 \mathrm{H})$.

${ }^{13}$ C-NMR (100 MHz, CDCl3): $\delta /$ ppm = 143.5, 139.1, 133.0 (d, $J=1.4$ Hz), 130.6 (q, $J=32.2$ Hz), 128.7, 128.6 (2C), 127.9, 126.3 (q, $J=3.8 \mathrm{~Hz}), 125.9$ (2C), 124.2 (q, $J=272.6 \mathrm{~Hz}), 123.4$ $(\mathrm{q}, J=3.8 \mathrm{~Hz}), 75.2,45.6$.

IR (Diamond-ATR, neat): $\tilde{v} / \mathrm{cm}^{-1}=1709,1450,1421,1360,1330,1221,1203,1162,1121$, 1096, 1075, 1052, 1029, 795, 758, 702, 666 .

MS (EI, 70 eV): m/z (\%) = 159 (30), 109 (18), 107 (93), 105 (18), 79 (100), 77 (52).

HRMS (EI-orbitrap): $m / z$ : [M - $\left.\mathrm{H}_{2} \mathrm{O}\right]$ calcd for $\mathrm{C}_{15} \mathrm{H}_{11} \mathrm{~F}_{3} 248.0813$; Found 248.0809. 


\section{2-(6-Chloropyridin-3-yl)-1-phenylethan-1-ol (13a)}<smiles>OC(Cc1ccc(Cl)nc1)c1ccccc1</smiles>

According to the TP1, a solution of 2-chloro-5-(iodomethyl)pyridine (10a, 0.20 M, $0.20 \mathrm{mmol}$, 1.0 equiv) and benzaldehyde (2a, $0.30 \mathrm{M}, 0.30 \mathrm{mmol}, 1.5$ equiv) in THF (total volume: $1.0 \mathrm{~mL}$ ) and a solution of $t \mathrm{BuLi}(0.50 \mathrm{M}$ in hexane, $0.50 \mathrm{mmol}, 2.5$ equiv) were prepared. The precooled solutions were mixed with an overall $10 \mathrm{~mL} / \mathrm{min}$ flowrate in a T-mixer. The combined stream passed a $0.02 \mathrm{~mL}$ reactor tube $\left(0.1 \mathrm{~s},-78^{\circ} \mathrm{C}\right)$ and was subsequently injected in an empty flask. Stirring was continued for $10 \mathrm{~min}$ at $25{ }^{\circ} \mathrm{C}$ before sat. aq. $\mathrm{NH}_{4} \mathrm{Cl}$ solution was added to quench the reaction mixture. The aqueous phase was extracted three times with EtOAc $(3 \times 30 \mathrm{~mL})$ and the combined organic phases were dried over anhydrous $\mathrm{Na}_{2} \mathrm{SO}_{4}$ and filtrated. After removal of the solvent, flash chromatographical purification (silica gel, isohexane:EtOAc $=95: 5 \rightarrow$ 9:1) afforded the title compound 13a as white crystals (41 $\mathrm{mg}, 0.18 \mathrm{mmol}, 92 \%$ yield).

${ }^{1}$ H-NMR (400 MHz, CDCl 3$): \delta / \mathrm{ppm}=8.15(\mathrm{~d}, J=2.5 \mathrm{~Hz}, 1 \mathrm{H}), 7.42(\mathrm{dd}, J=8.1,2.5 \mathrm{~Hz}$, 1H), $7.38-7.27(\mathrm{~m}, 5 \mathrm{H}), 7.21(\mathrm{dd}, J=8.2,0.7 \mathrm{~Hz}, 1 \mathrm{H}), 4.88(\mathrm{t}, J=6.5 \mathrm{~Hz}, 1 \mathrm{H}), 3.07-2.94$ (m, 2H), $2.03(\mathrm{~s}, 1 \mathrm{H})$.

${ }^{13}$ C-NMR (100 MHz, CDCl3): $\delta$ / ppm = 150.6, 149.8, 143.2, 140.2, 132.6, 128.8 (2C), 128.3, 126.0 (2C), 123.8, 74.9, 42.0.

IR (Diamond-ATR, neat): $\tilde{v} / \mathrm{cm}^{-1}=3345,2917,1586,1568,1459,1434,1407,1386,1312$, 1302, 1292, 1214, 1202, 1140, 1110, 1092, 1076, 1060, 1027, 1003, 826, 813, 763, 738, 699, 685.

MS (EI, 70 eV): m/z (\%) = 233 (10), 215 (12), 129 (30), 127 (100), 107 (39), 105 (13), 91 (13), 79 (43), 77 (36).

HRMS (EI-orbitrap): $m / z$ : [M] calcd for $\mathrm{C}_{13} \mathrm{H}_{12} \mathrm{ClNO} 233.0607$; Found 233.0610.

m.p. $\left({ }^{\circ} \mathbf{C}\right): 106.7-111.0$. 
2-(6-Chloro-2-fluoropyridin-3-yl)-1-(2,6-dichlorophenyl)ethan-1-ol (13b)<smiles>OC(Cc1ccc(Cl)nc1F)c1c(Cl)cccc1Cl</smiles>

According to the TP1, a solution of 6-chloro-2-fluoro-3-(iodomethyl)pyridine (10b, $0.20 \mathrm{M}$, $0.20 \mathrm{mmol}, 1.0$ equiv) and 2,6-dichlorobenzaldehyde (2i, $0.30 \mathrm{M}, 0.30 \mathrm{mmol}, 1.5$ equiv) in THF (total volume: $1.0 \mathrm{~mL}$ ) and a solution of $t \mathrm{BuLi}(0.50 \mathrm{M}$ in hexane, $0.50 \mathrm{mmol}, 2.5$ equiv) were prepared. The precooled solutions were mixed with an overall $10 \mathrm{~mL} / \mathrm{min}$ flowrate in a T-mixer. The combined stream passed a $0.02 \mathrm{~mL}$ reactor tube $\left(0.1 \mathrm{~s},-78^{\circ} \mathrm{C}\right)$ and was subsequently injected in an empty flask. Stirring was continued for $10 \mathrm{~min}$ at $25^{\circ} \mathrm{C}$ before sat. $a q . \mathrm{NH}_{4} \mathrm{Cl}$ solution was added to quench the reaction mixture. The aqueous phase was extracted three times with EtOAc $(3 \times 30 \mathrm{~mL})$ and the combined organic phases were dried over anhydrous $\mathrm{Na}_{2} \mathrm{SO}_{4}$ and filtrated. After removal of the solvent, flash chromatographical purification (silica gel, isohexane:EtOAc $=95: 5 \rightarrow$ 9:1) afforded the title compound 13b as a yellow oil $(39 \mathrm{mg}$, $0.12 \mathrm{mmol}, 61 \%$ yield).

${ }^{1}$ H-NMR (400 MHz, CDCl3): $\delta /$ ppm $=7.65(\mathrm{dd}, J=9.3,7.7,1 \mathrm{H}), 7.39-7.31(\mathrm{~m}, 2 \mathrm{H}), 7.25$ 7.18 (m, 2H), $5.72(\mathrm{~s}, 1 \mathrm{H}), 3.49$ (dd, $J=14.0,8.8,1 \mathrm{H}), 3.24$ (dd, $J=14.0,6.2,2 \mathrm{H})$.

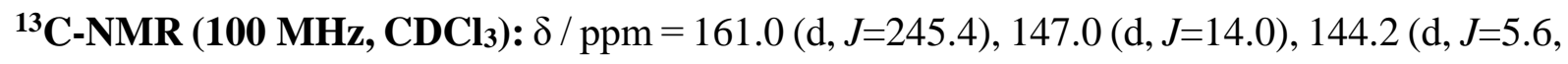
2C), 136.2 (2C), 134.4, 129.7 (2C), 121.8 (d, J=5.1), 118.1 (d, J=29.3), 71.5, 34.1 (d, J=2.7).

IR (Diamond-ATR, neat): $\tilde{v} / \mathrm{cm}^{-1}=3416,2931,1707,1601,1565,1435,1396,1315,1264$, 1202, 1182, 1139, 1098, 1088, 1049, 1003, 950, 912, 872, 821, 800, 778, 768, 750, 727, 678.

MS (EI, 70 eV): $m / z(\%)=177$ (27), 175 (40), 147 (29), 145 (100), 111 (23), 109 (12), 75 (17).

HRMS (EI-orbitrap): $m / z$ : [M - $\mathrm{H}_{2} \mathrm{O}$ ] calcd for $\mathrm{C}_{13} \mathrm{H}_{7} \mathrm{Cl}_{3} \mathrm{FN} 300.9628$; Found 300.9623. 
<smiles>COc1ccc(C(O)Cc2ccc(Cl)nc2SC)cc1</smiles>

According to the TP1, a solution of 6-chloro-3-(iodomethyl)-2-(methylthio)pyridine (10c, $0.20 \mathrm{M}, 0.20 \mathrm{mmol}, 1.0$ equiv) and $p$-methoxybenzaldehyde (2c, $0.30 \mathrm{M}, 0.30 \mathrm{mmol}, 1.5$ equiv) in THF (total volume: $1.0 \mathrm{~mL})$ and a solution of $t \mathrm{BuLi}(0.50 \mathrm{M}$ in hexane, $0.50 \mathrm{mmol}, 2.5$ equiv) were prepared. The precooled solutions were mixed with an overall $10 \mathrm{~mL} / \mathrm{min}$ flowrate in a Tmixer. The combined stream passed a $0.02 \mathrm{~mL}$ reactor tube $\left(0.1 \mathrm{~s},-78^{\circ} \mathrm{C}\right)$ and was subsequently injected in an empty flask. Stirring was continued for $10 \mathrm{~min}$ at $25^{\circ} \mathrm{C}$ before sat. aq. $\mathrm{NH}_{4} \mathrm{Cl}$ solution was added to quench the reaction mixture. The aqueous phase was extracted three times with EtOAc $(3 \times 30 \mathrm{~mL})$ and the combined organic phases were dried over anhydrous $\mathrm{Na}_{2} \mathrm{SO}_{4}$ and filtrated. After removal of the solvent, flash chromatographical purification (silica gel, isohexane:EtOAc $=95: 5 \rightarrow$ 9:1) afforded the title compound $\mathbf{1 3 c}$ as a white amorphous solid (34 mg, $0.11 \mathrm{mmol}, 55 \%$ yield).

${ }^{1}$ H-NMR (400 MHz, CDCl3): $\delta$ / ppm = 7.31 - 7.27 (m, 2H), 7.20 (d, J=7.8, 1H), 6.95 - 6.84 (m, 3H), 4.97 (t, J=6.6, 1H), 3.81 (s, 3H), $3.02-2.92$ (m, 2H), 2.59 (s, 3H), 1.95 (s, 1H).

${ }^{13}$ C-NMR (100 MHz, CDCl 3$): \delta$ / ppm = 159.4, 159.3, 149.0, 139.8, 136.0, 130.1, 127.1 (2C), 118.9, $114.0(2 \mathrm{C}), 72.2,55.4,41.8,13.6$.

IR (Diamond-ATR, neat): $\tilde{v} / \mathrm{cm}^{-1}=3342,3284,2923,2853,2838,1610,1575,1549,1511$, 1461, 1439, 1412, 1366, 1343, 1322, 1296, 1246, 1219, 1202, 1178, 1168, 1132, 1073, 1058, $1045,1026,1001,861,838,822,782,736,726,705$.

MS (EI, 70 eV): $m / z(\%)=175$ (35), 173 (100), 142 (25), 140 (80), 139 (15), 137 (69), 109 (51), 94 (24), 77 (10).

HRMS (EI-orbitrap): $m / z:$ : $\mathrm{M}-\mathrm{H}_{2} \mathrm{O}$ ] calcd for $\mathrm{C}_{15} \mathrm{H}_{14}$ CINOS 291.0485; Found 291.0480. m.p. $\left({ }^{\circ} \mathbf{C}\right): 86.8-89.1$. 
<smiles>Cc1nc(Cl)ccc1CC(O)c1c(Cl)cccc1Cl</smiles>

According to the TP1, a solution of 6-chloro-3-(iodomethyl)-2-(methylthio)pyridine (10c, $0.20 \mathrm{M}, 0.20 \mathrm{mmol}, 1.0$ equiv) and 2,6-dichlorobenzaldehyde (2i, $0.30 \mathrm{M}, 0.30 \mathrm{mmol}, 1.5$ equiv) in THF (total volume: $1.0 \mathrm{~mL})$ and a solution of $t \mathrm{BuLi}(0.50 \mathrm{M}$ in hexane, $0.50 \mathrm{mmol}, 2.5$ equiv) were prepared. The precooled solutions were mixed with an overall $10 \mathrm{~mL} / \mathrm{min}$ flowrate in a Tmixer. The combined stream passed a $0.02 \mathrm{~mL}$ reactor tube $\left(0.1 \mathrm{~s},-78^{\circ} \mathrm{C}\right)$ and was subsequently injected in an empty flask. Stirring was continued for 10 min at $25^{\circ} \mathrm{C}$ before sat. aq. $\mathrm{NH}_{4} \mathrm{Cl}$ solution was added to quench the reaction mixture. The aqueous phase was extracted three times with EtOAc $(3 \times 30 \mathrm{~mL})$ and the combined organic phases were dried over anhydrous $\mathrm{Na}_{2} \mathrm{SO}_{4}$ and filtrated. After removal of the solvent, flash chromatographical purification (silica gel, isohexane:EtOAc $=95: 5 \rightarrow$ 9:1) afforded the title compound 13d as a white amorphous solid (35 mg, $0.10 \mathrm{mmol}, 50 \%$ yield).

${ }^{1}$ H-NMR (400 MHz, CDCl3): $\delta$ / ppm = $7.28(\mathrm{~s}, 1 \mathrm{H}), 7.26(\mathrm{~s}, 1 \mathrm{H}), 7.21(\mathrm{~d}, J=7.8,1 \mathrm{H}), 7.13$ (dd, $J=8.5,7.5,1 \mathrm{H}), 6.89$ (d, $J=7.8,1 \mathrm{H}), 5.67$ (ddd, $J=9.9,8.9,5.9,1 \mathrm{H}), 3.44$ (dd, $J=14.4,8.9$, $1 \mathrm{H}), 3.12-3.03(\mathrm{~m}, 2 \mathrm{H}), 2.56(\mathrm{~s}, 3 \mathrm{H})$.

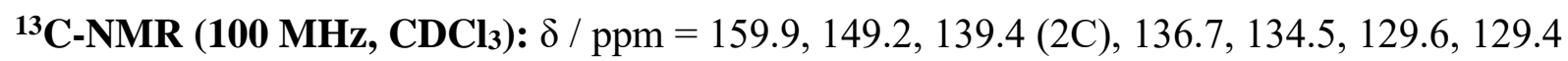
(2C), 129.3, 119.0, 71.3, 37.2, 13.7 .

IR (Diamond-ATR, neat): $\tilde{v} / \mathrm{cm}^{-1}=3402,2926,1577,1554,1435,1409,1364,1312,1297$, 1248, 1220, 1202, 1170, 1148, 1132, 1087, 1078, 1056, 971, 964, 862, 835, 811, 778, 766, 751, $722,710,666$.

MS (EI, 70 eV): $m / z(\%)=330(11), 328$ (11), 264 (10), 191 (25), 189 (79), 177 (17), 176 (16), 175 (25), 174 (46), 173 (19), 172 (52), 145 (16), 144 (11), 143 (53), 141 (100), 140 (13), 136 (11), 127 (13), 126 (23), 115 (13), 111 (14), 90 (13), 75 (16).

HRMS (EI-orbitrap): $m / z:[\mathrm{M}-\mathrm{H}]^{+}$calcd for $\mathrm{C}_{14} \mathrm{H}_{11} \mathrm{Cl}_{3} \mathrm{NOS}^{+}$345.9621; Found 345.9623 . m.p. $\left({ }^{\circ} \mathbf{C}\right): 115.8-120.3$. 


\section{2-(6-Chloro-2-(methylthio)pyridin-3-yl)-1-(4-chlorophenyl)ethan-1-ol (13e)}<smiles>Cc1nc(Cl)ccc1CC(O)c1ccc(Cl)cc1</smiles>

According to the TP1, a solution of 6-chloro-3-(iodomethyl)-2-(methylthio)pyridine (10c, $0.20 \mathrm{M}, 0.20 \mathrm{mmol}, 1.0$ equiv) and 4-chlorobenzaldehyde ( $2 \mathbf{k}, 0.30 \mathrm{M}, 0.30 \mathrm{mmol}, 1.5$ equiv) in THF (total volume: $1.0 \mathrm{~mL}$ ) and a solution of $t \mathrm{BuLi}(0.50 \mathrm{M}$ in hexane, $0.50 \mathrm{mmol}, 2.5$ equiv) were prepared. The precooled solutions were mixed with an overall $10 \mathrm{~mL} / \mathrm{min}$ flowrate in a Tmixer. The combined stream passed a $0.02 \mathrm{~mL}$ reactor tube $\left(0.1 \mathrm{~s},-78{ }^{\circ} \mathrm{C}\right)$ and was subsequently injected in an empty flask. Stirring was continued for $10 \mathrm{~min}$ at $25^{\circ} \mathrm{C}$ before sat. aq. $\mathrm{NH}_{4} \mathrm{Cl}$ solution was added to quench the reaction mixture. The aqueous phase was extracted three times with EtOAc $(3 \times 30 \mathrm{~mL})$ and the combined organic phases were dried over anhydrous $\mathrm{Na}_{2} \mathrm{SO}_{4}$ and filtrated. After removal of the solvent, flash chromatographical purification (silica gel, isohexane:EtOAc $=95: 5 \rightarrow$ 9:1) afforded the title compound 13e as a slightly yellow oil (30 mg, 0.09 mmol, $48 \%$ yield).

${ }^{1}$ H-NMR (400 MHz, CDCl3): $\delta / \mathrm{ppm}=7.35-7.28(\mathrm{~m}, 4 \mathrm{H}), 7.20(\mathrm{~d}, J=7.8,1 \mathrm{H}), 6.93(\mathrm{~d}$, $J=7.8,1 \mathrm{H}), 5.00(\mathrm{dd}, J=7.8,5.4,1 \mathrm{H}), 2.99-2.91(\mathrm{~m}, 2 \mathrm{H}), 2.60(\mathrm{~s}, 3 \mathrm{H}), 2.03(\mathrm{~s}, 1 \mathrm{H})$.

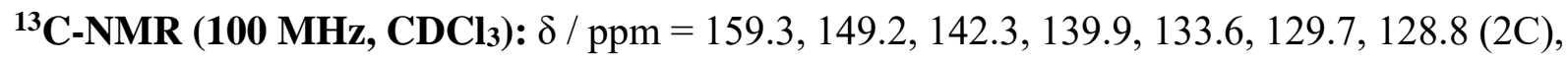
$127.2(2 \mathrm{C}), 119.0,71.9,42.0,13.6$.

IR (Diamond-ATR, neat): $\tilde{v} / \mathrm{cm}^{-1}=3416,3004,2927,1709,1576,1551,1513,1492,1416$, 1359, 1247, 1220, 1172, 1134, 1090, 1078, 1014, 860, 832.

MS (EI, 70 eV): m/z (\%) = 175 (25), 173 (72), 142 (33), 141 (15), 140 (100), 139 (19), 113 (15), 77 (20).

HRMS (EI-orbitrap): $m / z:[\mathrm{M}+\mathrm{H}]^{+}$calcd for $\mathrm{C}_{14} \mathrm{H}_{14} \mathrm{Cl}_{2} \mathrm{NOS}^{+} 314.0168$; Found 314.0168. 


\section{Typical procedures for the batch screening}

\section{Typical procedure for the sequential batch method}

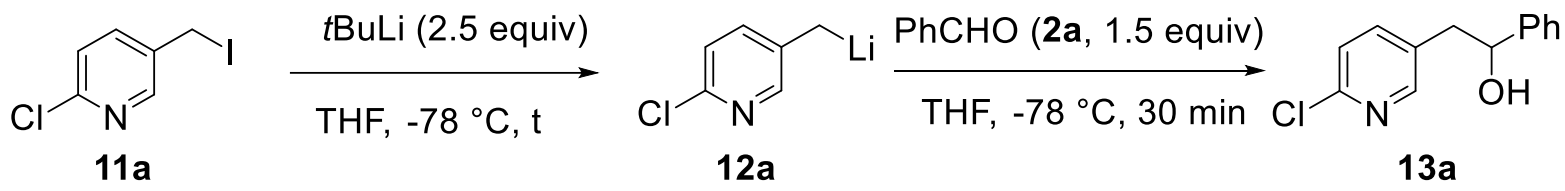

$0.20 \mathrm{mmol}$

To 2-chloro-5-(iodomethyl)pyridine (11a, $0.20 \mathrm{mmol}, 1.0$ equiv) in THF (1.0 mL) was added $t \mathrm{BuLi}$ $\left(1.0 \mathrm{~mL}, 0.50 \mathrm{M}\right.$ in hexane, $0.50 \mathrm{mmol}, 2.5$ equiv) at $-78{ }^{\circ} \mathrm{C}$. The reaction solution was stirred for an indicated time $\mathrm{t}\left(\mathrm{t}_{1}=1 \mathrm{~min}, \mathrm{t}_{2}=5 \mathrm{~min}, \mathrm{t}_{3}=30 \mathrm{~min}\right)$ at $-78^{\circ} \mathrm{C}$. Then, a solution of the benzaldehyde ( $2 \mathbf{a}$, $0.30 \mathrm{mmol}, 1.5$ equiv) in THF $(1.0 \mathrm{~mL})$ was added at $-78{ }^{\circ} \mathrm{C}$. The mixture was stirred at $-78{ }^{\circ} \mathrm{C}$ for further $30 \mathrm{~min}$ before it was allowed to warm to $25^{\circ} \mathrm{C}$ and quenched with sat. aq. $\mathrm{NH}_{4} \mathrm{Cl}$ solution. The crude mixture was filtrated over a pipet column containing silica and $\mathrm{MgSO}_{4}$ before it was analysed via GC-analysis.

\section{Typical procedure for the Barbier-type batch method}<smiles>Clc1ccc(CI)cn1</smiles>

$0.20 \mathrm{mmol}$

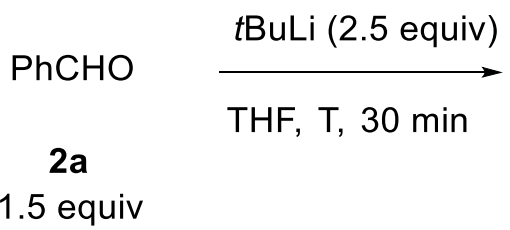<smiles>OC(Cc1ccc(Cl)nc1)c1ccccc1</smiles>

$13 a$

To 2-chloro-5-(iodomethyl)pyridine (11a, $0.20 \mathrm{mmol}, 1.0$ equiv) and benzaldehyde (2a, $0.30 \mathrm{mmol}$, 1.5 equiv) in THF $(1.0 \mathrm{~mL})$ at an indicated temperature $\mathrm{T}\left(\mathrm{T}_{1}=-20{ }^{\circ} \mathrm{C}, \mathrm{T}_{2}=-40{ }^{\circ} \mathrm{C}, \mathrm{T}_{3}=-78{ }^{\circ} \mathrm{C}\right)$ was added $t \mathrm{BuLi}(1.0 \mathrm{~mL}, 0.50 \mathrm{M}$ in hexane, $0.50 \mathrm{mmol}, 2.5$ equiv). The mixture was stirred at the indicated temperature for $30 \mathrm{~min}$ before it was quenched with sat. aq. $\mathrm{NH}_{4} \mathrm{Cl}$ solution. The crude mixture was filtrated over a pipet column containing silica and $\mathrm{MgSO}_{4}$ before it was analaysed via GC-analysis. The aqueous layer was extracted with EtOAc $(3 \times 30 \mathrm{~mL})$ and the combined organic phases were dried over anhydrous $\mathrm{Na}_{2} \mathrm{SO}_{4}$. Solvents were removed in vacuo and the crude product was purified by flash chromatography using suitable EtOAc and isohexane mixtures. 


\section{Batch screening results}

\section{1,2-Diphenylethan-1-ol (4a)}

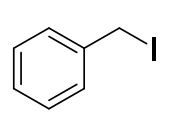

$1 \mathrm{a}$

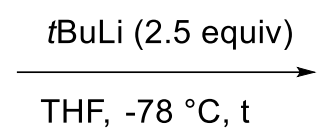

THF, $-78^{\circ} \mathrm{C}, \mathrm{t}$

$0.20 \mathrm{mmol}$

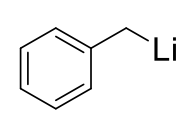

$3 a$

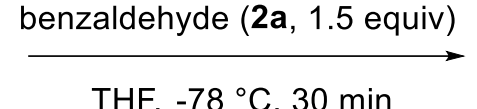

THF, $-78^{\circ} \mathrm{C}, 30 \mathrm{~min}$

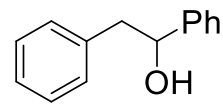

$4 a$

According to the typical procedure for the sequential batch approach, to a solution of $\mathbf{1 a}(44 \mathrm{mg}$, $0.20 \mathrm{mmol}, 1.0$ equiv) in THF $(1.0 \mathrm{~mL})$ was added $t \mathrm{BuLi}(1.0 \mathrm{~mL}, 0.50 \mathrm{mmol}, 0.50 \mathrm{M}$ in hexane, 2.5 equiv) at $-78^{\circ} \mathrm{C}$ the mixture was stirred for an indicated time $\mathrm{t}\left(\mathrm{t}_{1}=1 \mathrm{~min}, \mathrm{t}_{2}=5 \mathrm{~min}, \mathrm{t}_{3}=30 \mathrm{~min}\right)$ at $-78{ }^{\circ} \mathrm{C}$. Then, a solution of benzaldehyde $(\mathbf{2 a}, 32 \mathrm{mg}, 0.30 \mathrm{mmol}, 1.5$ equiv) in THF $(1.0 \mathrm{~mL})$ was added at $-78{ }^{\circ} \mathrm{C}$. The mixture was stirred at $-78{ }^{\circ} \mathrm{C}$ for further $30 \mathrm{~min}$ before it was allowed to warm to $25^{\circ} \mathrm{C}$ and quenched with sat. $a q . \mathrm{NH}_{4} \mathrm{Cl}$ solution.

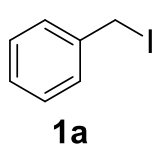

$0.20 \mathrm{mmol}$

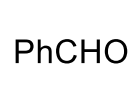

$2 a$

1.5 equiv

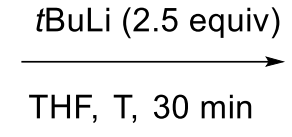

THF, T, $30 \mathrm{~min}$<smiles>OC(Cc1ccccc1)c1ccccc1</smiles>

$4 a$

According to the typical procedure for the Barbier-type batch reactions, to a solution of $\mathbf{1 a}$ (44 $\mathrm{mg}$, $0.20 \mathrm{mmol}, 1.0$ equiv) and benzaldehyde (2a, $32 \mathrm{mg}, 0.30 \mathrm{mmol}, 1.5$ equiv) in THF (1.0 mL), $t$ BuLi $\left(1.0 \mathrm{~mL}, 0.50 \mathrm{M}\right.$ in hexane, $0.50 \mathrm{mmol}, 2.5$ equiv) was added at an indicated temperature $\mathrm{T}\left(\mathrm{T}_{1}=-20{ }^{\circ} \mathrm{C}\right.$, $\mathrm{T}_{2}=-40^{\circ} \mathrm{C}, \mathrm{T}_{3}=-78{ }^{\circ} \mathrm{C}$ ). The mixture was stirred at the indicated temperature for $30 \mathrm{~min}$ before it was quenched with sat. aq. $\mathrm{NH}_{4} \mathrm{Cl}$ solution. The crude mixture was filtrated over a pipet column containing silica and $\mathrm{MgSO}_{4}$ before it was analaysed via gas chromatography. For entry 5, the aqueous layer was extracted with EtOAc $(3 \times 30 \mathrm{~mL})$ and the combined organic phases were dried over anhydrous $\mathrm{Na}_{2} \mathrm{SO}_{4}$. Solvents were removed in vacuo and the crude product was purified by flash chromatography using isohexane:EtOAc 9:1. 4a was obtained as colorless crystals (16 mg, $0.08 \mathrm{mmol}, 40 \%$ yield).

Table SI 4: Batch screening of reaction between 1a and 2a affording product $4 \mathbf{a}$.

\begin{tabular}{|c|c|c|c|c|c|}
\hline entry & procedure & $\begin{array}{c}\mathrm{t} \\
{[\mathrm{min}]}\end{array}$ & $\begin{array}{c}\mathrm{T} \\
{\left[{ }^{\circ} \mathrm{C}\right]}\end{array}$ & $\begin{array}{c}\text { conv. } \\
{[\%]}\end{array}$ & $\begin{array}{c}\text { GC-yield } \\
\text { [\%] }\end{array}$ \\
\hline 1 & sequential & 1 & -78 & $>95$ & $<5$ \\
\hline 2 & sequential & 5 & -78 & $>95$ & $<5$ \\
\hline 3 & sequential & 30 & -78 & $>95$ & $<5$ \\
\hline 4 & Barbier-type & 30 & -20 & $>95$ & 32 \\
\hline 5 & Barbier- type & 30 & -40 & $>95$ & $40^{[\mathrm{a}]}$ \\
\hline 6 & Barbier- type & 30 & -78 & $>95$ & 31 \\
\hline
\end{tabular}

GC-yields were calculated by normation of the isolated yield. ${ }^{[\mathrm{a}]}$ Isolated yield. 


\section{2-(3-Methoxybenzyl)adamantan-2-ol (7g)}

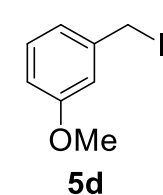

5d

$0.20 \mathrm{mmol}$

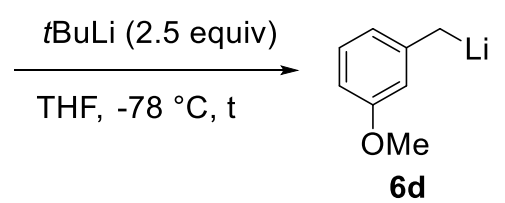

6d

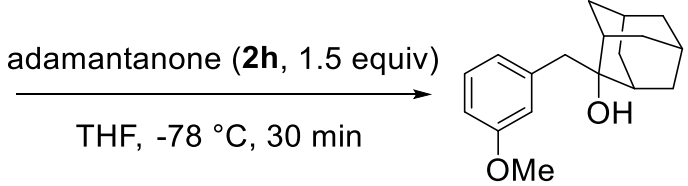

$7 \mathrm{~g}$

According to the typical procedure for the sequential batch approach, to a solution of $\mathbf{5 d}(50 \mathrm{mg}$, $0.20 \mathrm{mmol}, 1.0$ equiv) in THF $(1.0 \mathrm{~mL})$ was added $t \mathrm{BuLi}(1.0 \mathrm{~mL}, 0.50 \mathrm{M}$ in hexane, $0.50 \mathrm{mmol}$, 2.5 equiv) at $-78{ }^{\circ} \mathrm{C}$. The mixture was stirred for an indicated time $\mathrm{t}\left(\mathrm{t}_{1}=1 \mathrm{~min}, \mathrm{t}_{2}=5 \mathrm{~min}, \mathrm{t}_{3}=30 \mathrm{~min}\right)$ at $-78{ }^{\circ} \mathrm{C}$. Then, a solution of adamantanone $(2 \mathbf{h}, 45 \mathrm{mg}, 0.30 \mathrm{mmol}, 1.5$ equiv) in THF $(1.0 \mathrm{~mL})$ was added at $-78{ }^{\circ} \mathrm{C}$. The mixture was stirred at $-78{ }^{\circ} \mathrm{C}$ for further $30 \mathrm{~min}$ before it was allowed to warm to $25^{\circ} \mathrm{C}$ and quenched with sat. $a q . \mathrm{NH}_{4} \mathrm{Cl}$ solution.

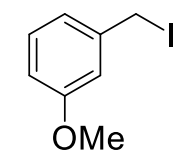

$5 d$

$0.20 \mathrm{mmol}$

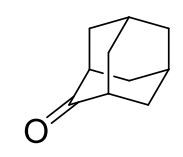

$2 \mathrm{~h}$

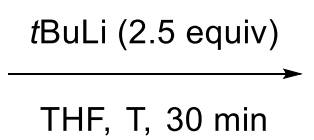

THF, T, $30 \mathrm{~min}$

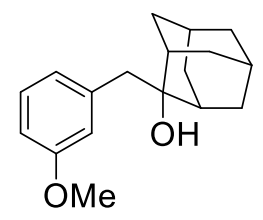

$7 \mathrm{~g}$

According to the typical procedure for the Barbier-type batch reactions, to a solution of $\mathbf{5 d}(50 \mathrm{mg}$, $0.20 \mathrm{mmol}, 1.0$ equiv) and adamantanone ( $\mathbf{2} \mathbf{h}, 45 \mathrm{mg}, 0.30 \mathrm{mmol}, 1.5$ equiv) in THF (1.0 mL), $t \mathrm{BuLi}$ $\left(1.0 \mathrm{~mL}, 0.50 \mathrm{M}\right.$ in hexane, $0.50 \mathrm{mmol}, 2.5$ equiv) was added at an indicated temperature $\mathrm{T}\left(\mathrm{T}_{1}=-20^{\circ} \mathrm{C}\right.$, $\mathrm{T}_{2}=-40^{\circ} \mathrm{C}, \mathrm{T}_{3}=-78^{\circ} \mathrm{C}$ ). The mixture was stirred at the indicated temperature for $30 \mathrm{~min}$ before it was quenched with sat. $a q$. $\mathrm{NH}_{4} \mathrm{Cl}$ solution. The crude mixture was filtrated over a pipet column containing silica and $\mathrm{MgSO}_{4}$ before it was analaysed via gas chromatography. For entry 4, the aqueous layer was extracted with EtOAc $(3 \times 30 \mathrm{~mL})$ and the combined organic phases were dried over anhydrous $\mathrm{Na}_{2} \mathrm{SO}_{4}$. Solvents were removed in vacuo and the crude product was purified by flash chromatography using isohexane:EtOAc 9:1.7g was obtained as colorless crystals ( $8 \mathrm{mg}, 0.03 \mathrm{mmol}, 15 \%$ yield).

Table SI 5: Batch screening of reaction between $5 \mathrm{~d}$ and $2 \mathrm{~h}$ affording product $\mathbf{7 g}$.

\begin{tabular}{cccccc} 
entry & procedure & $\mathrm{t}$ & $\mathrm{T}$ & \multicolumn{2}{c}{ conv. GC-yield } \\
1 & sequential & {$[\mathrm{min}]$} & {$\left[{ }^{\circ} \mathrm{C}\right]$} & {$[\%]$} & {$[\%]$} \\
2 & sequential & 1 & -78 & $>95$ & $<5$ \\
3 & sequential & 5 & -78 & $>95$ & $<5$ \\
4 & Barbier-type & 30 & -78 & $>95$ & $<5$ \\
5 & Barbier- type & 30 & -20 & $>95$ & $15^{[\mathrm{a}]}$ \\
6 & Barbier- type & 30 & -40 & $>95$ & 14 \\
& & 30 & -78 & $>95$ & 13
\end{tabular}

GC-yields were calculated by normation using the isolated yield. ${ }^{[a]}$ Isolated yield. 


\section{2-(6-Chloropyridin-3-yl)-1-phenylethan-1-ol (13a)}

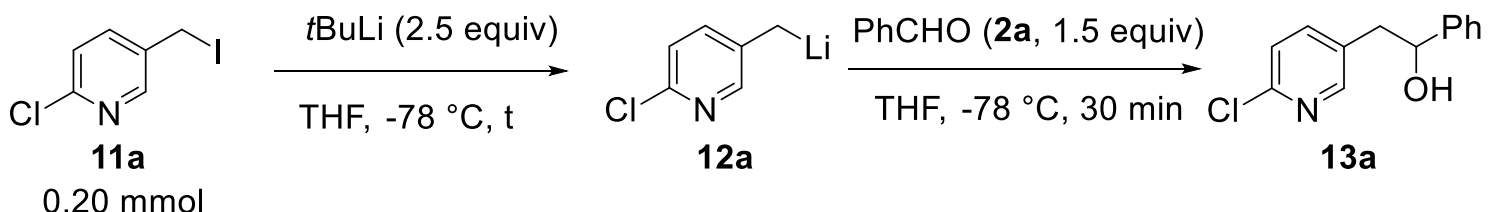

According to the typical procedure for the sequential batch approach, to a solution of 11a $(51 \mathrm{mg}$, $0.20 \mathrm{mmol}, 1.0$ equiv) in THF $(1.0 \mathrm{~mL})$ was added $t \mathrm{BuLi}(1.0 \mathrm{~mL}, 0.50 \mathrm{M}$ in hexane, $0.50 \mathrm{mmol}$, 2.5 equiv) at $-78{ }^{\circ} \mathrm{C}$ the mixture was stirred for a time $\mathrm{t}\left(\mathrm{t}_{1}=1 \mathrm{~min}, \mathrm{t}_{2}=5 \mathrm{~min}, \mathrm{t}_{3}=30 \mathrm{~min}\right)$ at $-78{ }^{\circ} \mathrm{C}$. Then, a solution of benzaldehyde (2a, $32 \mathrm{mg}, 0.30 \mathrm{mmol}, 1.5$ equiv) in THF (1.0 mL) was added at -78 ${ }^{\circ} \mathrm{C}$. The mixture was stirred at this temperature for further $30 \mathrm{~min}$ before it was allowed to warm to $25{ }^{\circ} \mathrm{C}$ and quenched with sat. $a q . \mathrm{NH}_{4} \mathrm{Cl}$ solution.
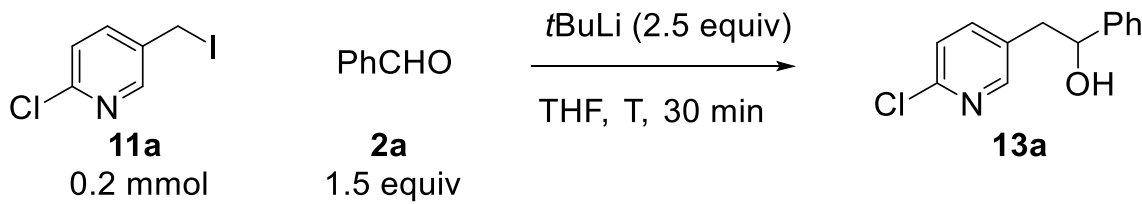

According to the typical procedure for the Barbier-type batch reactions, to a solution of $11 \mathrm{a}(51 \mathrm{mg}$, $0.20 \mathrm{mmol}, 1.0$ equiv) and benzaldehyde ( $\mathbf{2 a}, 32 \mathrm{mg}, 0.3 \mathrm{mmol}, 1.5$ equiv) in THF (1.0 mL), $t \mathrm{BuLi}$ $\left(1.0 \mathrm{~mL}, 0.50 \mathrm{M}\right.$ in hexane, $0.50 \mathrm{mmol}, 2.5$ equiv) was added at an indicated temperature $\mathrm{T}\left(\mathrm{T}_{1}=-20^{\circ} \mathrm{C}\right.$, $\mathrm{T}_{2}=-40^{\circ} \mathrm{C}, \mathrm{T}_{3}=-78^{\circ} \mathrm{C}$ ). The mixture was stirred at the indicated temperature for $30 \mathrm{~min}$ before it was quenched with sat. $a q . \mathrm{NH}_{4} \mathrm{Cl}$ solution. The crude mixture was filtrated over a pipet column containing silica and $\mathrm{MgSO}_{4}$ before it was analysed via GC-analysis.

Table SI 6: Batch screening of reaction between 11a and 2a affording product 13a.

\begin{tabular}{|c|c|c|c|c|c|}
\hline entry & procedure & $\begin{array}{c}\mathrm{t} \\
{[\mathrm{min}]}\end{array}$ & $\begin{array}{c}\mathrm{T} \\
{\left[{ }^{\circ} \mathrm{C}\right]}\end{array}$ & $\begin{array}{c}\text { conv. } \\
{[\%]}\end{array}$ & $\begin{array}{c}\text { GC-yield } \\
\text { [\%] }\end{array}$ \\
\hline 1 & sequential & 1 & -78 & $>95$ & $<5$ \\
\hline 2 & sequential & 5 & -78 & $>95$ & $<5$ \\
\hline 3 & sequential & 30 & -78 & $>95$ & $<5$ \\
\hline 4 & Barbier-type & 30 & -20 & $>95$ & $<5$ \\
\hline 5 & Barbier- type & 30 & -40 & $>95$ & $<5$ \\
\hline 6 & Barbier- type & 30 & -78 & $>95$ & $<5$ \\
\hline
\end{tabular}




\section{Synthesis of starting materials}

\section{Typical procedure for the preparation of (hetero)benzylic iodides}

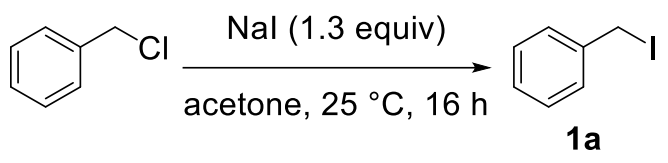

(Chloromethyl)benzene (1.26 g, $10.0 \mathrm{mmol}, 1.0$ equiv) was dissolved in dry acetone $(120 \mathrm{~mL})$ and $\mathrm{NaI}$ ( $2.18 \mathrm{~g}, 13.0 \mathrm{mmol}, 1.3$ equiv) was added in a flame dried flask covered in aluminium foil. The mixture was stirred for $16 \mathrm{~h}$ at $25{ }^{\circ} \mathrm{C}$. Solvents were evaporated in vacuo. The crude residues were transferred with $\mathrm{Et}_{2} \mathrm{O}(30 \mathrm{~mL})$ into a separatory funnel and washed with sat. $a q$. $\mathrm{Na}_{2} \mathrm{~S}_{2} \mathrm{O}_{3}(30 \mathrm{~mL})$. The aqueous layer was extracted with $\mathrm{Et}_{2} \mathrm{O}(3 \times 30 \mathrm{~mL})$. The combined organic layers were dried over $\mathrm{MgSO}_{4}$. After filtration, a copper turning was added to the filtrate and the solvents were removed in vacuo to obtain the desired product as a slightly yellow oil $(1.79 \mathrm{~g}, 8.2 \mathrm{mmol}, 82 \%$ yield$)$, which was stored at $-24{ }^{\circ} \mathrm{C}$ together with a copper turning. 
(Iodomethyl)benzene (1a)

$\mathrm{CH}_{2} \mathrm{I}$

(Chloromethyl)benzene (1.26 g, $10.0 \mathrm{mmol}, 1.0$ equiv) was dissolved in dry acetone $(120 \mathrm{~mL})$ and $\mathrm{NaI}(2.18 \mathrm{~g}, 13.0 \mathrm{mmol}, 1.3 \mathrm{equiv})$ was added in a flame dried flask covered in aluminium foil. The mixture was stirred for $16 \mathrm{~h}$ at $25^{\circ} \mathrm{C}$. Solvents were evaporated in vacuo. The crude residues were transferred with $\mathrm{Et}_{2} \mathrm{O}(30 \mathrm{~mL})$ into a separatory funnel and washed with sat. $a q$. $\mathrm{Na}_{2} \mathrm{~S}_{2} \mathrm{O}_{3}(30 \mathrm{~mL})$. The aqueous layer was extracted with $\mathrm{Et}_{2} \mathrm{O}(3 \times 30 \mathrm{~mL})$. The combined organic layers were dried over $\mathrm{MgSO}_{4}$. After filtration, a copper turning was added to the filtrate and the solvents were removed in vacuo to obtain the desired product as a slightly yellow oil (1.79 g, $8.2 \mathrm{mmol}, 82 \%$ yield), which was stored at $-24{ }^{\circ} \mathrm{C}$ together with a copper turning.

${ }^{1}$ H-NMR (400 MHz, CDCl3): $\delta$ / ppm = $7.41-7.36$ (m, 2H), $7.33-7.21$ (m, 3H), 4.46 (s, 2H). ${ }^{13}$ C-NMR (100 MHz, CDCl3): $\delta$ / ppm = 139.4, 129.0 (2C), 128.9 (2C), 128.0, 5.8.

The spectra matched with those reported in the literature. ${ }^{1}$

\section{1-(Tert-butyl)-4-(iodomethyl)benzene (1b)}<smiles>CC(C)(C)c1ccc(CI)cc1</smiles>

1-(Tert-butyl)-4-(chloromethyl)benzene (1.82 g, $10.0 \mathrm{mmol}, 1.0$ equiv) was dissolved in dry acetone $(120 \mathrm{~mL})$ and $\mathrm{NaI}(2.18 \mathrm{~g}, 13.0 \mathrm{mmol}, 1.3$ equiv $)$ was added in a flame dried flask covered in aluminium foil. The mixture was stirred for $16 \mathrm{~h}$ at $25^{\circ} \mathrm{C}$. Solvents were evaporated in vacuo. The crude residues were transferred with $\mathrm{Et}_{2} \mathrm{O}(30 \mathrm{~mL})$ into a separatory funnel and washed with sat. $a q . \mathrm{Na}_{2} \mathrm{~S}_{2} \mathrm{O}_{3}(30 \mathrm{~mL})$. The aqueous layer was extracted with $\mathrm{Et}_{2} \mathrm{O}(3 \times 30 \mathrm{~mL})$. The combined organic layers were dried over $\mathrm{MgSO}_{4}$. After filtration, a copper turning was added to the filtrate and the solvents were removed in vacuo to obtain the desired product as yellow oil (2.11 g, $7.7 \mathrm{mmol}, 77 \%$ yield), which was stored at $-24{ }^{\circ} \mathrm{C}$ together with a copper turning.

${ }^{1}$ H-NMR (400 MHz, CDCl3): $\delta$ / ppm = 7.32 (s, 4H), 4.46 (s, 2H), 1.31 (s, 9H).

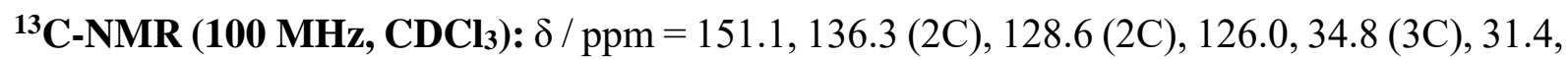
6.2.

The spectra matched with those reported in the literature. ${ }^{2}$

\footnotetext{
${ }^{1}$ Combe, S. H.; Hosseini A.; Song, L.; Hausmann, H.; Schreiner, P. R. Org .Lett. 2017, 19, 6156.

${ }^{2}$ Nugent, J.; Arroniz, C.; Shire, B. R.; Sterling, A. J.; Pickford, H. D.; Wong, M. L. J.; Mansfield, S. J.; Caputo, D. F. J.; Owen, B.; Mousseau, J. J.; Duarte, F.; Anderson, E. A. ACS Catal. 2019, 9, 9568.
} 


\section{1-(Iodomethyl)-4-isopropylbenzene (1c)}<smiles>CC(C)c1ccc(CI)cc1</smiles>

1-(Chloromethyl)-4-isopropylbenzene (1.68 g, $10.0 \mathrm{mmol}, 1.0$ equiv) was dissolved in dry acetone $(120 \mathrm{~mL})$ and $\mathrm{NaI}(2.18 \mathrm{~g}, 13.0 \mathrm{mmol}, 1.3 \mathrm{equiv})$ was added in a flame dried flask covered in aluminium foil. The mixture was stirred for $16 \mathrm{~h}$ at $25^{\circ} \mathrm{C}$. Solvents were evaporated in vacuo. The crude residues were transferred with $\mathrm{Et}_{2} \mathrm{O}(30 \mathrm{~mL})$ into a separatory funnel and washed with sat. aq. $\mathrm{Na}_{2} \mathrm{~S}_{2} \mathrm{O}_{3}(30 \mathrm{~mL})$. The aqueous layer was extracted with $\mathrm{Et}_{2} \mathrm{O}(3 \times 30 \mathrm{~mL})$. The combined organic layers were dried over $\mathrm{MgSO}_{4}$. After filtration, a copper turning was added to the filtrate and the solvents were removed in vacuo to obtain the desired product as brown oil (2.18 g, $8.4 \mathrm{mmol}, 84 \%$ yield), which was stored at $-24{ }^{\circ} \mathrm{C}$ together with a copper turning.

${ }^{1} \mathbf{H}-\mathbf{N M R}(400 \mathrm{MHz}, \mathbf{C D C l} 3): \delta / \mathrm{ppm}=7.34-7.28(\mathrm{~m}, 2 \mathrm{H}), 7.18-7.13(\mathrm{~m}, 2 \mathrm{H}), 4.46(\mathrm{~s}, 2 \mathrm{H})$, $2.96-2.81(\mathrm{~m}, J=7.0,1 \mathrm{H}), 1.24(\mathrm{dd}, J=7.0,5.5,6 \mathrm{H})$.

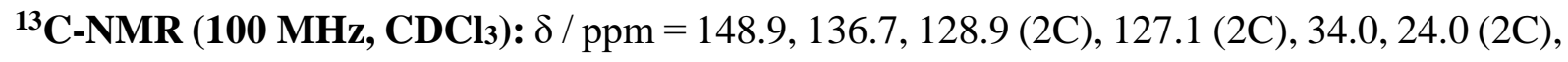
6.3 .

The spectra matched with those reported in the literature. ${ }^{3}$

\section{3-(Hydroxymethyl)phenol}<smiles>O=Cc1cccc(O)c1</smiles>

(1.0 equiv)

$$
\underset{\mathrm{H}_{2} \mathrm{O}, 25^{\circ} \mathrm{C}, 1 \mathrm{~h}}{\stackrel{\mathrm{NaBH}_{4}(2.0 \text { equiv })}{\longrightarrow}}
$$

$85 \%$ yield

According to literature ${ }^{4}$, 2-hydroxybenzaldehyde (3.96 g, $32.4 \mathrm{mmol}, 1.0$ equiv) was dissolved in water $(90 \mathrm{~mL}) . \mathrm{NaBH}_{4}(2.45 \mathrm{~g}, 64.8 \mathrm{mmol}, 2.0$ equiv) was added portionwise and the mixture was stirred for $1 \mathrm{~h}$ at $25{ }^{\circ} \mathrm{C}$. The reaction mixture was cooled to $0{ }^{\circ} \mathrm{C}$ and $6 \mathrm{M} \mathrm{HCl}$ was added until $\mathrm{pH} 5$ was reached. The aqueous layer was extracted with EtOAc $(3 \times 50 \mathrm{~mL})$ and the combined organic layers were dried over $\mathrm{MgSO}_{4}$. Evaporation of the solvents gave the title compound as colorless oil (3.40 g, $27.4 \mathrm{mmol}$, 85\% yield) which was used without further purification.

\footnotetext{
${ }^{3}$ Ruso, J. S.; Rajendiran, N.; Kumaran, R. S. J. Korean Chem. Soc. 2014, 58, 39.

${ }^{4}$ Guiso, M.; Betrow, A.; Marra, C. Eur. J. Org. Chem. 2008, 11, 1967.
} 
${ }^{1} \mathbf{H}-N M R(400$ MHz, CDCl 3$): \delta / p p m=7.16(\mathrm{t}, J=7.8,1 \mathrm{H}), 6.85$ (ddd, $\left.J=7.6,1.6,0.9,1 \mathrm{H}\right)$, $6.80(\mathrm{t}, J=2.2,1 \mathrm{H}), 6.70(\mathrm{ddd}, J=8.1,2.7,0.9,1 \mathrm{H}), 4.93(\mathrm{~s}, 1 \mathrm{H}), 4.60$ (d, J=4.9, 2H), 1.65 (t, $J=5.8,1 \mathrm{H})$.

${ }^{13}$ C-NMR (100 MHz, CDCl3): $\delta$ / ppm = 156.0, 142.8, 130.0, 119.3, 114.7, 113.9, 65.2.

The spectra matched with those reported in the literature. ${ }^{5}$

\section{3-(Chloromethyl)phenol}<smiles>OCc1cccc(O)c1</smiles>

(1.0 equiv)

$$
\frac{\mathrm{SOCl}_{2}(3.0 \text { equiv) }}{\mathrm{DMF}, 25^{\circ} \mathrm{C}, 18 \mathrm{~h}}
$$<smiles>Oc1cccc(CCl)c1</smiles>

$29 \%$ yield

To a solution of 3-(hydroxymethyl)phenol (3.43 g, $27.6 \mathrm{mmol}, 1.0$ equiv) in DMF (50.0 mL) was added $\mathrm{SOCl}_{2}(6.0 \mathrm{~mL}, 82.9 \mathrm{mmol}, 3.0$ equiv $)$ at $0{ }^{\circ} \mathrm{C}$. The reaction mixture was stirred for $18 \mathrm{~h}$ at $25^{\circ} \mathrm{C}$. Water $(50 \mathrm{~mL})$ was added to the mixture and the aqueous layer was extracted with EtOAc $(3 \times 50 \mathrm{~mL})$. The combined organic layers were washed with sat. $a q$. LiCl solution $(5 \times 100 \mathrm{~mL})$ and dried over $\mathrm{MgSO}_{4}$. Solvents were removed in vacuo and the residue was purified by flash column chromatography (isohexane:EtOAc $=9: 1$ ) to obtain the title compound as yellow oil (1.15 g, $8.1 \mathrm{mmol}, 29 \%$ yield).

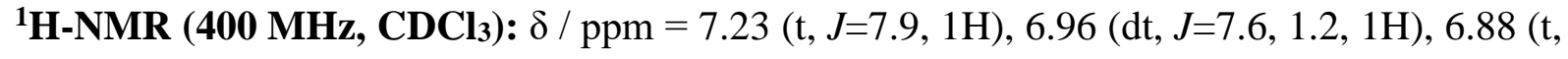
$J=2.1,1 \mathrm{H}), 6.79$ (ddd, $J=8.1,2.6,0.9,1 \mathrm{H}), 4.54$ (s, 2H).

${ }^{13}$ C-NMR (100 MHz, CDCl3): $\delta$ / ppm = 156.2, 139.1, 130.0, 120.7, 115.6 (2C), 46.1.

The spectra matched with those reported in the literature. ${ }^{6}$

\section{Tert-butyl(3-(chloromethyl)phenoxy)dimethylsilane}

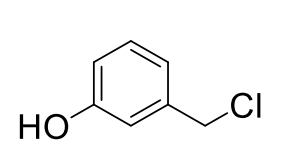

(1.0 equiv)

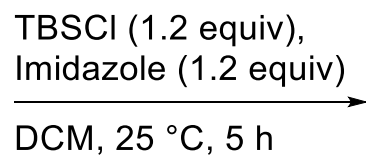

$\mathrm{DCM}, 25^{\circ} \mathrm{C}, 5 \mathrm{~h}$

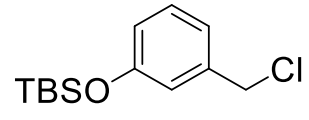

$72 \%$ yield

To a solution of 3-(Chloromethyl)phenol (1.15 g, $8.1 \mathrm{mmol}, 1.0$ equiv) and imidazole ( $0.66 \mathrm{~g}$, $9.7 \mathrm{mmol}, 1.2$ equiv) in DCM (40 mL) TBSCl (1. $46 \mathrm{~g}, 9.7 \mathrm{mmol}, 1.2$ equiv) was added portionwise. The mixture was stirred for $5 \mathrm{~h}$ at $25^{\circ} \mathrm{C}$. After the reaction was completed, water $(50 \mathrm{~mL})$ was added and the aqueous layer was extracted with DCM $(3 \times 50 \mathrm{~mL})$. The combined organic layers were dried over $\mathrm{MgSO}_{4}$. Solvents were removed in vacuo and the crude residue

\footnotetext{
${ }^{5}$ Aoun, S.; Sierocki, P.; Lebreton, Mathé-Allainmat, M. Synthesis 2019, 51, 3556.

${ }^{6}$ Pouliot, M.-F.; Mahé, O.; Hamel, J.-D., Desroches, J.; Paquin, J.-F. Org. Lett. 2012, 14, 5428.
} 
was purified by column chromatography to obtain the title compound as a colorless, amorphous solid (1.50 g, $5.8 \mathrm{mmol}, 72 \%$ yield).

${ }^{1}$ H-NMR (400 MHz, CDCl3): $\delta /$ ppm = $7.21(\mathrm{t}, J=7.9,1 \mathrm{H}), 6.97(\mathrm{dt}, J=7.7,1.2,1 \mathrm{H}), 6.87(\mathrm{t}$, $J=2.1,1 \mathrm{H}), 6.79$ (ddd, $J=8.1,2.5,1.0,1 \mathrm{H}), 4.53$ (s, 2H), 0.98 (s, 9H), 0.20 (s, 6H).

${ }^{13}$ C-NMR (100 MHz, CDCl3): $\delta$ / ppm = 155.9, 138.9, 129.7, 121.4, 120.3, 120.1, 46.1, 25.7 (3C), 18.2, $-4.4(2 \mathrm{C})$.

The spectra matched with those reported in the literature. ${ }^{7}$

\section{Tert-butyl(3-(iodomethyl)phenoxy)dimethylsilane (5a)}<smiles>[R5]S(=O)(=O)c1cccc(CI)c1</smiles>

Tert-butyl(3-(chloromethyl)phenoxy)dimethylsilane $(2.57 \mathrm{~g}, 10.0 \mathrm{mmol}, 1.0$ equiv) was dissolved in dry acetone $(120 \mathrm{~mL})$ and $\mathrm{NaI}(2.18 \mathrm{~g}, 13.0 \mathrm{mmol}, 1.3 \mathrm{equiv})$ was added in a flame dried flask covered in aluminium foil. The mixture was stirred for $16 \mathrm{~h}$ at $25{ }^{\circ} \mathrm{C}$. Solvents were evaporated in vacuo. The crude residues were transferred with $\mathrm{Et}_{2} \mathrm{O}(30 \mathrm{~mL})$ into a separatory funnel and washed with sat. $a q . \mathrm{Na}_{2} \mathrm{~S}_{2} \mathrm{O}_{3}(30 \mathrm{~mL})$. The aqueous layer was extracted with $\mathrm{Et}_{2} \mathrm{O}$ $(3 \times 30 \mathrm{~mL})$. The combined organic layers were dried over $\mathrm{MgSO}_{4}$. After filtration, a copper turning was added to the filtrate and the solvents were removed in vacuo to obtain the desired product as a slightly brown amorphous solid (1.95 g, $5.6 \mathrm{mmol}, 56 \%$ yield), which was stored at $-24{ }^{\circ} \mathrm{C}$ together with a copper turning.

${ }^{1}$ H-NMR (400 MHz, CDCl3): $\delta /$ ppm = $7.14(\mathrm{t}, J=7.9,1 \mathrm{H}), 6.96(\mathrm{dq}, J=7.6,1.7,1.3,1 \mathrm{H}), 6.85$ (t, $J=2.1,1 \mathrm{H}), 6.71$ (ddd, $J=8.1,2.4,1.0,1 \mathrm{H}), 4.40$ (s, 2H), 0.98 (d, $J=1.1,9 \mathrm{H}), 0.20$ (d, $J=1.3$, $6 \mathrm{H})$.

${ }^{13}$ C-NMR (100 MHz, CDCl3): $\delta$ / ppm = 155.9, 140.7, 129.9, 121.8, 120.7, 119.9, 25.8 (3C), $18.3,5.7,-4.3(2 \mathrm{C})$.

The spectra matched with those reported in the literature. ${ }^{8}$

\section{(4-(Iodomethyl)phenyl)(methyl)sulfane (5b)}<smiles>CS(=O)(=O)c1ccc(CI)cc1</smiles>

(4-(Chloromethyl)phenyl)(methyl)sulfane (1.72 g, $10.0 \mathrm{mmol}, 1.0$ equiv) was dissolved in dry acetone $(120 \mathrm{~mL})$ and $\mathrm{NaI}(2.18 \mathrm{~g}, 13.0 \mathrm{mmol}, 1.3 \mathrm{equiv})$ was added in a flame dried flask

\footnotetext{
${ }^{7}$ Huy, P. H.; Filbrich, I. Chem. Eur. J. 2018, 24, 7410.

${ }^{8}$ Berkowitz, D., B.; McFadden, J. M.; Sloss, M. K. J. Org. Chem. 2000, 65, 2907.
} 
covered in aluminium foil. The mixture was stirred for $16 \mathrm{~h}$ at $25^{\circ} \mathrm{C}$. Solvents were evaporated in vacuo. The crude residues were transferred with $\mathrm{Et}_{2} \mathrm{O}(30 \mathrm{~mL})$ into a separatory funnel and washed with sat. aq. $\mathrm{Na}_{2} \mathrm{~S}_{2} \mathrm{O}_{3}(30 \mathrm{~mL})$. The aqueous layer was extracted with $\mathrm{Et}_{2} \mathrm{O}(3 \times 30 \mathrm{~mL})$. The combined organic layers were dried over $\mathrm{MgSO}_{4}$. After filtration, a copper turning was added to the filtrate and the solvents were removed in vacuo to obtain the desired product as yellow amorphous solid (2.43 g, $9.2 \mathrm{mmol}, 92 \%$ yield), which was stored at $-24{ }^{\circ} \mathrm{C}$ together with a copper turning.

${ }^{1}$ H-NMR (400 MHz, CDCl3): $\delta$ / ppm = $7.32-7.27$ (m, 2H), $7.19-7.14(\mathrm{~m}, 2 \mathrm{H}), 4.45(\mathrm{~s}, 2 \mathrm{H})$, $2.47(\mathrm{~s}, 3 \mathrm{H})$.

${ }^{13}$ C-NMR (100 MHz, CDCl3): $\delta$ / ppm = 138.7, 136.1, 129.3 (2C), 126.8 (2C), 15.8, 5.9.

MS (EI, $70 \mathrm{eV}): m / z(\%)=137(100), 122(25), 121(12)$.

HRMS (EI-orbitrap): $m / z:[\mathrm{M}-\mathrm{H}]^{+}$calcd for $\mathrm{C}_{8} \mathrm{H}_{8} \mathrm{IS}^{+}$262.9386; Found 262.9384.

m.p. $\left({ }^{\circ} \mathbf{C}\right): 60.4-62.1$.

\section{1-(Iodomethyl)-4-methoxybenzene (5c)}<smiles>COc1ccc(CI)cc1</smiles>

1-(Chloromethyl)-4-methoxybenzene (1.56 g, $10.0 \mathrm{mmol}, 1.0$ equiv) was dissolved in dry acetone $(120 \mathrm{~mL})$ and $\mathrm{NaI}(2.18 \mathrm{~g}, 13.0 \mathrm{mmol}, 1.3 \mathrm{equiv})$ was added in a flame dried flask covered in aluminium foil. The mixture was stirred for $16 \mathrm{~h}$ at $25^{\circ} \mathrm{C}$. Solvents were evaporated in vасио. The crude residues were transferred with $\mathrm{Et}_{2} \mathrm{O}(30 \mathrm{~mL})$ into a separatory funnel and washed with sat. $a q$. $\mathrm{Na}_{2} \mathrm{~S}_{2} \mathrm{O}_{3}(30 \mathrm{~mL})$. The aqueous layer was extracted with $\mathrm{Et}_{2} \mathrm{O}(3 \times 30 \mathrm{~mL})$. The combined organic layers were dried over $\mathrm{MgSO}_{4}$. After filtration, a copper turning was added to the filtrate and the solvents were removed in vacuo to obtain the desired product as a slightly yellow liquid $(2.11 \mathrm{~g}, 8.5 \mathrm{mmol}, 85 \%$ yield $)$, which was stored at $-24{ }^{\circ} \mathrm{C}$ together with a copper turning.

${ }^{1} \mathbf{H}-\mathbf{N M R}(400 \mathrm{MHz}, \mathbf{C D C l} 3): \delta / \mathrm{ppm}=7.35-7.29(\mathrm{~m}, 2 \mathrm{H}), 6.85-6.80$ (m, 2H), 4.48 (s, 2H), $3.80(\mathrm{~s}, 3 \mathrm{H})$.

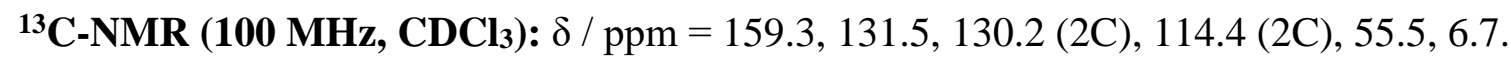

The spectra matched with those reported in the literature. ${ }^{9}$

\footnotetext{
${ }^{9}$ Iranpoor, N.; Firouzabadi, H.; Jamalian, A.; Kazemi, F. Tetrahedron, 2005, 61, 5699.
} 


\section{1-(Iodomethyl)-3-methoxybenzene (5d)}<smiles>COc1cccc(CI)c1</smiles>

1-(Chloromethyl)-3-methoxybenzene (1.56 g, $10.0 \mathrm{mmol}, 1.0$ equiv) was dissolved in dry acetone $(120 \mathrm{~mL})$ and $\mathrm{NaI}(2.18 \mathrm{~g}, 13.0 \mathrm{mmol}, 1.3$ equiv) was added in a flame dried flask covered in aluminium foil. The mixture was stirred for $16 \mathrm{~h}$ at $25^{\circ} \mathrm{C}$. Solvents were evaporated in vacuo. The crude residues were transferred with $\mathrm{Et}_{2} \mathrm{O}(30 \mathrm{~mL})$ into a separatory funnel and washed with sat. aq. $\mathrm{Na}_{2} \mathrm{~S}_{2} \mathrm{O}_{3}(30 \mathrm{~mL})$. The aqueous layer was extracted with $\mathrm{Et}_{2} \mathrm{O}(3 \times 30 \mathrm{~mL})$. The combined organic layers were dried over $\mathrm{MgSO}_{4}$. After filtration, a copper turning was added to the filtrate and the solvents were removed in vacuo to obtain the desired product as slightly yellow liquid $(2.01 \mathrm{~g}, 8.1 \mathrm{mmol}, 81 \%$ yield $)$, which was stored at $-24{ }^{\circ} \mathrm{C}$ together with a copper turning.

${ }^{1}$ H-NMR (400 MHz, CDCl3): $\delta /$ ppm = $7.21(\mathrm{t}, J=7.9,1 \mathrm{H}), 6.97(\mathrm{dt}, J=7.7,1.3,1 \mathrm{H}), 6.91(\mathrm{t}$, $J=2.1,1 \mathrm{H}), 6.79$ (ddd, $J=8.2,2.6,0.9,1 \mathrm{H}), 4.43$ (s, 2H), 3.81 (s, 3H).

${ }^{13}$ C-NMR (100 MHz, CDCl3): $\delta /$ ppm = 159.8, 140.8, 130.0, 121.2, 114.3, 113.8, 55.4, 5.7.

The spectra matched with those reported in the literature. ${ }^{10}$

\section{1-Fluoro-2-(iodomethyl)benzene (8a)}<smiles>Fc1ccccc1CI</smiles>

1-(Chloromethyl)-2-fluorobenzene ( $1.45 \mathrm{~g}, 10.0 \mathrm{mmol}, 1.0$ equiv) was dissolved in dry acetone $(120 \mathrm{~mL})$ and $\mathrm{NaI}(2.18 \mathrm{~g}, 13.0 \mathrm{mmol}, 1.3 \mathrm{equiv})$ was added in a flame dried flask covered in aluminium foil. The mixture was stirred for $16 \mathrm{~h}$ at $25^{\circ} \mathrm{C}$. Solvents were evaporated in vacuo. The crude residues were transferred with $\mathrm{Et}_{2} \mathrm{O}(30 \mathrm{~mL})$ into a separatory funnel and washed with sat. aq. $\mathrm{Na}_{2} \mathrm{~S}_{2} \mathrm{O}_{3}(30 \mathrm{~mL})$. The aqueous layer was extracted with $\mathrm{Et}_{2} \mathrm{O}(3 \times 30 \mathrm{~mL})$. The combined organic layers were dried over $\mathrm{MgSO}_{4}$. After filtration, a copper turning was added to the filtrate and the solvents were removed in vacuo to obtain the desired product as a brown oil (1.84 g, $7.8 \mathrm{mmol}, 78 \%$ yield), which was stored at $-24{ }^{\circ} \mathrm{C}$ together with a copper turning. ${ }^{1} \mathbf{H}-\mathbf{N M R}\left(400 \mathrm{MHz}, \mathbf{C D C l}_{3}\right): \delta / \mathrm{ppm}=7.26(\mathrm{td}, J=8.0,5.9,1 \mathrm{H}), 7.15(\mathrm{dt}, J=7.7,1.3,1 \mathrm{H})$, 7.08 (dt, $J=9.5,2.1,1 \mathrm{H}), 6.94$ (tdd, $J=8.4,2.6,1.0,1 \mathrm{H}), 4.42(\mathrm{~s}, 2 \mathrm{H})$.

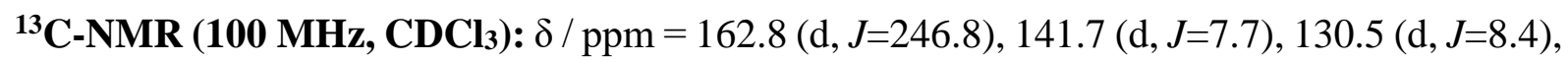
124.5 (d, $J=2.9), 115.9$ (d, $J=22.0), 115.1$ (d, $J=21.2), 4.1$ (d, $J=2.2)$.

\footnotetext{
${ }^{10}$ Rafiee, M.; Wang, F.; Hruszkewycz, D. P.; Stahl, S. S. J. Am. Chem. Soc. 2018, 140, 22.
} 
The spectra matched with those reported in the literature. ${ }^{11}$

\section{1-Chloro-2-(iodomethyl)benzene (8b)}<smiles>Clc1ccccc1CI</smiles>

1-Chloro-2-(chloromethyl)benzene ( $1.61 \mathrm{~g}, 10.0 \mathrm{mmol}, 1.0$ equiv) was dissolved in dry acetone $(120 \mathrm{~mL})$ and $\mathrm{NaI}(2.18 \mathrm{~g}, 13.0 \mathrm{mmol}, 1.3 \mathrm{equiv})$ was added in a flame dried flask covered in aluminium foil. The mixture was stirred for $16 \mathrm{~h}$ at $25^{\circ} \mathrm{C}$. Solvents were evaporated in vacuo. The crude residues were transferred with $\mathrm{Et}_{2} \mathrm{O}(30 \mathrm{~mL})$ into a separatory funnel and washed with sat. aq. $\mathrm{Na}_{2} \mathrm{~S}_{2} \mathrm{O}_{3}(30 \mathrm{~mL})$. The aqueous layer was extracted with $\mathrm{Et}_{2} \mathrm{O}(3 \times 30 \mathrm{~mL})$. The combined organic layers were dried over $\mathrm{MgSO}_{4}$. After filtration, a copper turning was added to the filtrate and the solvents were removed in vacuo to obtain the desired product as a slightly yellow oil $\left(2.17 \mathrm{~g}, 8.6 \mathrm{mmol}, 86 \%\right.$ yield), which was stored at $-24{ }^{\circ} \mathrm{C}$ together with a copper turning.

${ }^{1}$ H-NMR (400 MHz, CDCl3): $\delta$ / ppm = 7.44 - 7.38 (m, 1H), 7.38 - 7.31 (m, 1H), 7.25 - 7.18 $(\mathrm{m}, 2 \mathrm{H}), 4.53(\mathrm{~s}, 2 \mathrm{H})$.

${ }^{13}$ C-NMR (100 MHz, CDCl $)$ : $\delta$ / ppm = 136.9, 134.0, 130.7, 130.3, 129.6, 127.5, 2.6.

The spectra matched with those reported in the literature. ${ }^{12}$

\section{1-(Iodomethyl)-3-(trifluoromethyl)benzene (8c)}<smiles>FC(F)(F)c1cccc(CI)c1</smiles>

1-(Chloromethyl)-3-(trifluoromethyl)benzene (1.95 g, $10.0 \mathrm{mmol}, 1.0$ equiv) was dissolved in dry acetone $(120 \mathrm{~mL})$ and $\mathrm{NaI}(2.18 \mathrm{~g}, 13.0 \mathrm{mmol}, 1.3$ equiv) was added in a flame dried flask covered in aluminium foil. The mixture was stirred for $16 \mathrm{~h}$ at $25^{\circ} \mathrm{C}$. Solvents were evaporated in vасио. The crude residues were transferred with $\mathrm{Et}_{2} \mathrm{O}(30 \mathrm{~mL})$ into a separatory funnel and washed with sat. aq. $\mathrm{Na}_{2} \mathrm{~S}_{2} \mathrm{O}_{3}(30 \mathrm{~mL})$. The aqueous layer was extracted with $\mathrm{Et}_{2} \mathrm{O}(3 \times 30 \mathrm{~mL})$. The combined organic layers were dried over $\mathrm{MgSO}_{4}$. After filtration, a copper turning was added to the filtrate and the solvents were removed in vacuo to obtain the desired product as

\footnotetext{
${ }^{11}$ Ayres, J.A.; Ashford M. W.; Stöckl, Y.; Prudhomme, V.; Ling, K. B.; Platts, J. A.; Morrill, L. C. Org. Lett. 2017, 19, 3835 .

${ }^{12}$ Combe, S. H.; Hosseini A.; Song, L.; Hausmann, H.; Schreiner, P. R. Org .Lett. 2017, 19, 6156.
} 
yellow amorphous solid (1.52 g, $5.3 \mathrm{mmol}, 53 \%$ yield), which was stored at $-24{ }^{\circ} \mathrm{C}$ together with a copper turning.

${ }^{1} \mathbf{H}-N M R\left(400 \mathrm{MHz}, \mathbf{C D C l}_{3}\right): \delta / \mathrm{ppm}=7.62(\mathrm{q}, J=1.9,1 \mathrm{H}), 7.56(\mathrm{dt}, J=7.6,1.6,1 \mathrm{H}), 7.53-$ $7.48(\mathrm{~m}, 1 \mathrm{H}), 7.42(\mathrm{t}, J=7.7,1 \mathrm{H}), 4.47(\mathrm{~s}, 2 \mathrm{H})$.

${ }^{13}$ C-NMR (100 MHz, CDCl3): $\delta / \mathrm{ppm}=140.4,132.2$ (d, J=1.4), 131.3 (q, J=32.6), 129.5, 125.5 (q, $J=3.8), 124.8$ (q, $J=3.8), 124.0$ (q, $J=272.3 \mathrm{~Hz}$ ), 3.6.

MS (EI, 70 eV): $m / z(\%)=160(10), 159$ (100), 109 (20).

HRMS (EI-orbitrap): $m / z:[\mathrm{M}-\mathrm{H}]^{+}$calcd for $\mathrm{C}_{8} \mathrm{H}_{5} \mathrm{~F}_{3} \mathrm{I}$ 284.9383; Found 284.9380.

m.p. $\left({ }^{\circ} \mathbf{C}\right): 32.5-33.7$.

\section{2-Chloro-5-(iodomethyl)pyridine (11a)}<smiles>Clc1ccc(CI)cn1</smiles>

2-Chloro-5-(chloromethyl)pyridine (1.62 g, $10.0 \mathrm{mmol}, 1.0$ equiv) was dissolved in dry acetone $(120 \mathrm{~mL})$ and $\mathrm{NaI}$ (2.18 g, $13.0 \mathrm{mmol}, 1.3$ equiv) was added in a flame dried flask covered in aluminium foil. The mixture was stirred for $16 \mathrm{~h}$ at $25^{\circ} \mathrm{C}$. Solvents were evaporated in vacuo. The crude residues were transferred with $\mathrm{Et}_{2} \mathrm{O}(30 \mathrm{~mL})$ into a separatory funnel and washed with sat. aq. $\mathrm{Na}_{2} \mathrm{~S}_{2} \mathrm{O}_{3}(30 \mathrm{~mL})$. The aqueous layer was extracted with $\mathrm{Et}_{2} \mathrm{O}(3 \times 30 \mathrm{~mL})$. The combined organic layers were dried over $\mathrm{MgSO}_{4}$. After filtration, a copper turning was added to the filtrate and the solvents were removed in vacuo to obtain the desired product as yellow crystalls (1.82 g, $7.2 \mathrm{mmol}, 72 \%$ yield), which was stored at $-24{ }^{\circ} \mathrm{C}$ together with a copper turning.

${ }^{1}$ H-NMR (400 MHz, CDCl3): $\delta$ / ppm = $8.40(\mathrm{~d}, J=2.6,1 \mathrm{H}), 7.67(\mathrm{dd}, J=8.2,2.6,1 \mathrm{H}), 7.28$ (d, $J=8.2,1 \mathrm{H}), 4.38(\mathrm{~s}, 2 \mathrm{H})$.

${ }^{13}$ C-NMR (100 MHz, $\left.\mathbf{C D C l} 3\right): \delta$ / ppm = 150.7, 149.2, 139.4, 134.5, 124.6, -0.8.

MS (EI, 70 eV): $m / z(\%)=128(34), 126(100), 90(19)$.

HRMS (EI-orbitrap): $m / z$ : [M] calcd for $\mathrm{C}_{6} \mathrm{H}_{5} \mathrm{ClIN} 251.9066$; Found 251.9066.

m.p. $\left({ }^{\circ} \mathbf{C}\right): 55.9-58.1$.

\section{6-Chloro-2-fluoro-3-iodopyridine}

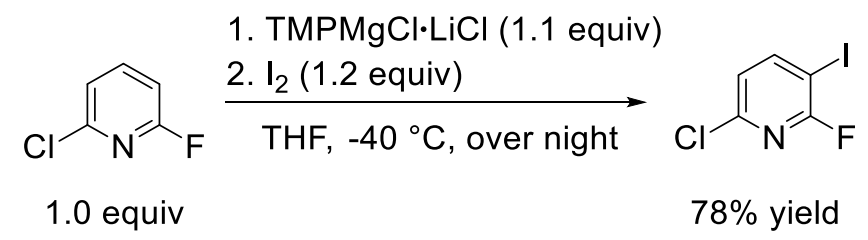


To a solution of 2-chloro-6-fluoropyridine ( $8.90 \mathrm{~g}, 67.7 \mathrm{mmol}$. 1.0 equiv) in THF $(37.5 \mathrm{~mL})$ was slowly added TMPMgCl$\cdot \mathrm{LiCl}$ solution (1.45 $\mathrm{M}$ in THF, $52.0 \mathrm{~mL}, 75.4 \mathrm{mmol}, 1.1$ equiv) at $-40{ }^{\circ} \mathrm{C}$. The mixture was stirred at $-40{ }^{\circ} \mathrm{C}$ for $2 \mathrm{~h}$. A solution of iodine $(20.8 \mathrm{~g}, 82.0 \mathrm{mmol}$, 1.2 equiv) in THF (40.0 mL) was added slowly to the reaction mixture. The mixture was allowed to warm to $25{ }^{\circ} \mathrm{C}$ and stirred for $16 \mathrm{~h}$. The reaction was quenched with sat. aq. $\mathrm{Na}_{2} \mathrm{~S}_{2} \mathrm{O}_{3}$ $(30 \mathrm{~mL})$ solution. The aqueous layer was extracted with EtOAc $(3 \times 30 \mathrm{~mL})$. The combined organic layers were dried over $\mathrm{Na}_{2} \mathrm{SO}_{4}$ and filtrated. Solvents were removed in vacuo. Flash chromatographical purification (isohexane $\rightarrow$ isohexane:EtOAc 9:1) afforded the title compound as a colorless amorphous solid (13.6 g, $52.8 \mathrm{mmol}, 78 \%$ yield).

${ }^{1}$ H-NMR (400 MHz, CDCl3): $\delta / \mathrm{ppm}=8.08(\mathrm{t}, J=8.0,1 \mathrm{H}), 7.03(\mathrm{dd}, J=8.0,1.2,1 \mathrm{H})$.

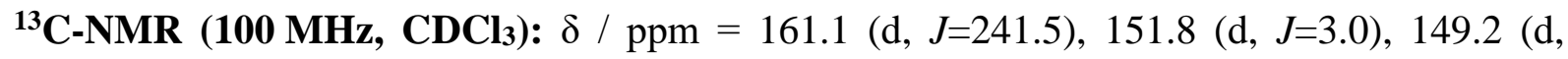
$J=12.6), 123.4$ (d, $J=5.4), 73.3$ (d, $J=41.4)$.

MS (EI, 70 eV): $m / z(\%)=259$ (32), 257 (100), 158 (11), 130 (14), 110 (11).

HRMS (EI-orbitrap): $m / z$ : [M] calcd for $\mathrm{C}_{5} \mathrm{H}_{2} \mathrm{CIFIN} 256.8904$; Found 256.8899.

The spectra matched with those reported in the literature. ${ }^{13}$

\section{1-(6-Chloro-2-fluoropyridin-3-yl)-N,N-dimethylmethanamine}

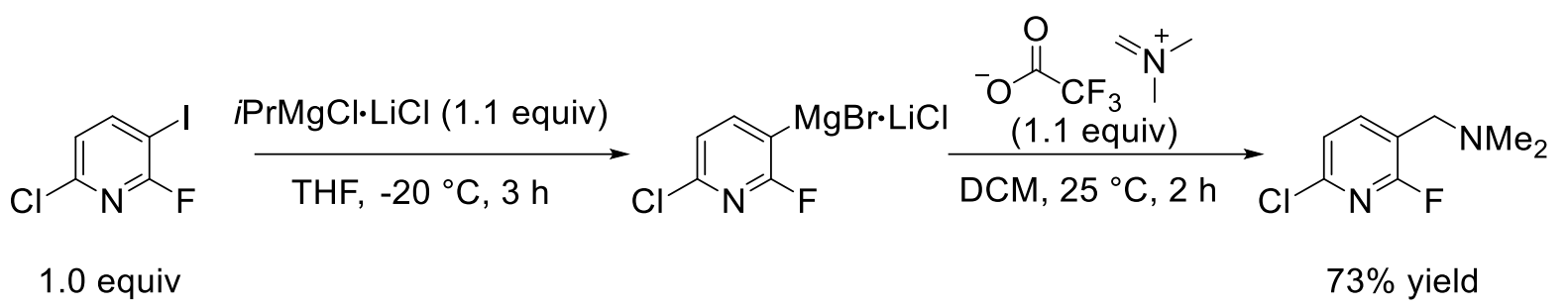

According to literature ${ }^{15}$, to 6-chloro-2-fluoro-3-iodopyridine ( $7.70 \mathrm{~g}, 30 \mathrm{mmol}, 1.0$ equiv) in THF $(30.0 \mathrm{~mL})$ was added $i \operatorname{PrMgCl} \cdot \mathrm{LiCl}(1.22 \mathrm{M}$ in THF, $27.0 \mathrm{~mL}, 33 \mathrm{mmol}, 1.1$ equiv) at $-30{ }^{\circ} \mathrm{C}$. The mixture was stirred at $-30{ }^{\circ} \mathrm{C}$ for $2 \mathrm{~h}$. To a solution of $N, N, N^{\prime}, N^{\prime}$-tetramethyl methylene diamine $\left(4.5 \mathrm{~mL}, 33 \mathrm{mmol}, 1.1\right.$ equiv) in DCM $(33 \mathrm{~mL})$ at $0{ }^{\circ} \mathrm{C}$ was carefully added trifluoroacetic anhydride $(4.65 \mathrm{~mL}, 33 \mathrm{mmol}, 1.1 \mathrm{equiv})$ and stirred for $30 \mathrm{~min}$ at $0{ }^{\circ} \mathrm{C}$. The methylene(dimethyl)iminium trifluoroacetate solution was added dropwise at $0{ }^{\circ} \mathrm{C}$ to the prepared Grignard reagent and stirred for $2 \mathrm{~h}$ at $25^{\circ} \mathrm{C}$. The mixture was quenched with sat. $a q$. $\mathrm{NaHCO}_{3}(30 \mathrm{~mL})$. The aqueous phase was extracted with EtOAc $(3 \times 30 \mathrm{~mL})$ and the combined organic layer were dried over anhydrous $\mathrm{Na}_{2} \mathrm{SO}_{4}$ and filtrated. After removal of the solvent, flash chromatographical purification (silica gel, DCM:EtOH = 96:4) afforded the title compound (4.14 g, $22 \mathrm{mmol}, 73 \%$ yield) as a brown oil.

\footnotetext{
${ }^{13}$ Barl, N. M.; Sansiaume-Dagousset, E.; Monzón, G.; Wagner, A. J.; Knochel, P. Org. Lett. 2014, $16,2422$.
} 
${ }^{1}$ H-NMR (400 MHz, CDCl3): $\delta / \mathrm{ppm}=7.90-7.82(\mathrm{~m}, 1 \mathrm{H}), 7.23(\mathrm{dd}, J=7.8,1.0,1 \mathrm{H}), 3.50$ (s, 2H), $2.30(\mathrm{~s}, 6 \mathrm{H})$.

${ }^{13}$ C-NMR (100 MHz, CDCl $): \delta$ / ppm = 160.6 (d, $\left.J=245.8\right), 147.4,144.13$ (d, J=5.3), 122.0, $121.9,55.5$ (d, J=2.7), 45.2 (2C).

MS (EI, 70 eV): $m / z(\%)=190$ (14), 189 (19), 188 (43), 187 (63), 146 (33), 144 (100), 108 (11), 58 (31).

HRMS (EI-orbitrap): $m / z$ : [M] calcd for $\mathrm{C}_{8} \mathrm{H}_{10} \mathrm{ClFN}_{2}$ 188.0517; Found 188.0510.

The spectra matched with those reported in the literature. ${ }^{14}$

\section{6-Chloro-3-(chloromethyl)-2-fluoropyridine}<smiles>CN(C)Cc1ccc(Cl)nc1F</smiles>

(1.0 equiv)<smiles>CCOC(=O)Cl</smiles>

(1.1 equiv)
$\overrightarrow{\mathrm{CHCl}_{3}, 0^{\circ} \mathrm{C} \text {, over night }}$<smiles>Fc1nc(Cl)ccc1CCl</smiles>

$47 \%$ yield

According to literature ${ }^{15}$, to a solution of 6-chloro-3-(chloromethyl)-2-(methylthio)pyridine (4.14 g, $22.0 \mathrm{mmol}, 1.0$ equiv) in $\mathrm{CHCl}_{3}(22 \mathrm{~mL})$ was added ethyl chloroformate $(2.3 \mathrm{~mL}$, $24.0 \mathrm{mmol}, 1.1$ equiv) at $0{ }^{\circ} \mathrm{C}$. The mixture was allowed to slowly warm up to $25^{\circ} \mathrm{C}$ and stirred for $16 \mathrm{~h}$. The mixture was quenched with $\mathrm{H}_{2} \mathrm{O}$ and the aqueous layer was extracted with EtOAc $(3 \times 30 \mathrm{~mL})$. The combined organic phases were dried over anhydrous $\mathrm{Na}_{2} \mathrm{SO}_{4}$ and filtrated, solvents were removed in vacuo and the crude product was purified by flash column chromatography (isohexane $\rightarrow$ isohexane:EtOAc 9:1). The title compound was obtained as a colorless amorphous solid (2.32 g, $8.6 \mathrm{mmol}, 47 \%$ yield).

${ }^{1}$ H-NMR (400 MHz, CDCl3): $\delta$ / ppm = $7.84(\mathrm{ddd}, J=9.2,7.8,0.6,1 \mathrm{H}), 7.29-7.25(\mathrm{~m}, 1 \mathrm{H})$, 4.59 (s, 2H).

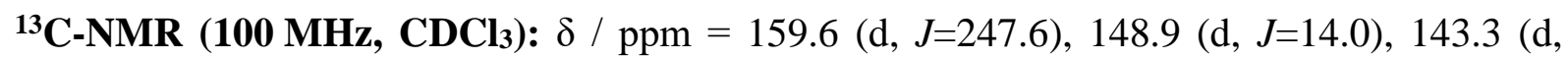
$J=4.2), 122.3$ (d, $J=5.2), 118.4$ (d, $J=27.9), 38.0$ (d, $J=1.5)$.

MS (EI, 70 eV): $m / z(\%)=179(11), 146(33), 144$ (100), 108 (10).

HRMS (EI-orbitrap): $m / z$ : [M] calcd for $\mathrm{C}_{6} \mathrm{H}_{4} \mathrm{Cl}_{2} \mathrm{FN}$ 178.9705; Found 178.9697.

The spectra matched with those reported in the literature. ${ }^{16}$

\footnotetext{
${ }^{14}$ Barl, N. M.; Sansiaume-Dagousset, E.; Monzón, G.; Wagner, A. J.; Knochel, P. Org. Lett. 2014, 16, 2422.

${ }^{15}$ Barl, N. M.; Sansiaume-Dagousset, E.; Monzón, G.; Wagner, A. J.; Knochel, P. Org. Lett. 2014, 16, 2422.

${ }^{16}$ Barl, N. M.; Sansiaume-Dagousset, E.; Monzón, G.; Wagner, A. J.; Knochel, P. Org. Lett. 2014, 16, 2422.
} 


\section{6-Chloro-2-fluoro-3-(iodomethyl)pyridine (11b)}<smiles>Fc1nc(Cl)ccc1CI</smiles>

6-Chloro-3-(chloromethyl)-2-fluoropyridine (1.80 g, $10.0 \mathrm{mmol}, 1.0$ equiv) was dissolved in dry acetone $(120 \mathrm{~mL})$ and $\mathrm{NaI}(2.18 \mathrm{~g}, 13.0 \mathrm{mmol}, 1.3$ equiv) was added in a flame dried flask covered in aluminium foil. The mixture was stirred for $16 \mathrm{~h}$ at $25^{\circ} \mathrm{C}$. Solvents were evaporated in vacuo. The crude residues were transferred with $\mathrm{Et}_{2} \mathrm{O}(30 \mathrm{~mL})$ into a separatory funnel and washed with sat. $a q . \mathrm{Na}_{2} \mathrm{~S}_{2} \mathrm{O}_{3}(30 \mathrm{~mL})$. The aqueous layer was extracted with $\mathrm{Et}_{2} \mathrm{O}(3 \times 30 \mathrm{~mL})$. The combined organic layers were dried over $\mathrm{MgSO}_{4}$. After filtration, a copper turning was added to the filtrate and the solvents were removed in vacuo to obtain the desired product as yellow oil (1.79 g, $6.6 \mathrm{mmol}, 66 \%$ yield), which was stored at $-24{ }^{\circ} \mathrm{C}$ together with a copper turning.

${ }^{1}$ H-NMR (400 MHz, CDCl3): $\delta / \mathrm{ppm}=7.74(\mathrm{dd}, J=9.5,7.8,1 \mathrm{H}), 7.20(\mathrm{dd}, J=7.8,0.9,1 \mathrm{H})$, $4.35(\mathrm{~s}, 2 \mathrm{H})$.

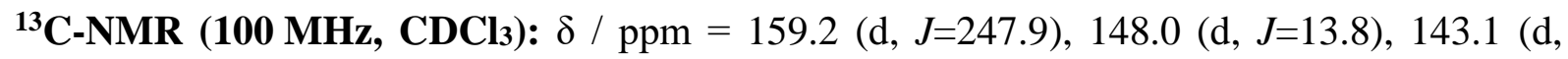
$J=4.3$ ), 122.4 (d, $J=5.2), 120.7$ (d, $J=28.0),-6.7$ (d, $J=1.9$ ).

MS (EI, 70 eV): $m / z(\%)=146(33), 144$ (100), 108 (11).

HRMS (EI-orbitrap): $m / z:[\mathrm{M}-\mathrm{H}]^{+}$calcd for $\mathrm{C}_{6} \mathrm{H}_{3} \mathrm{ClFIN}^{+}$269.8977; Found 269.8974.

\section{6-Chloro-3-(iodomethyl)-2-(methylthio)pyridine (11c)}
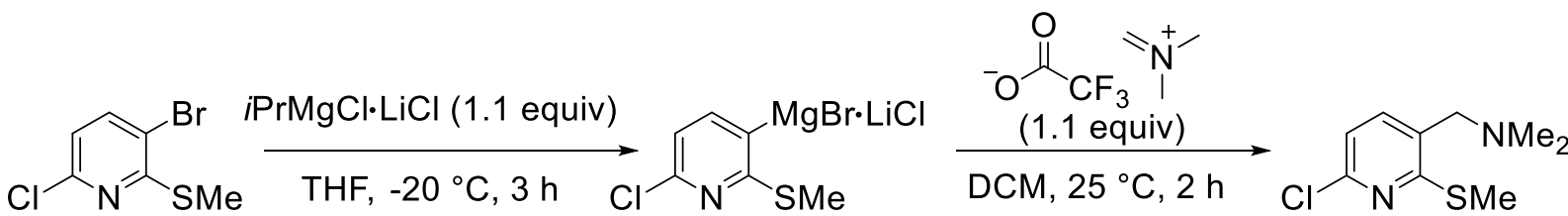

(1.0 equiv)

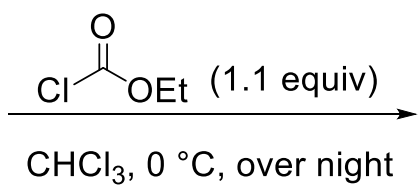<smiles>CSc1nc(Cl)ccc1CCl</smiles>
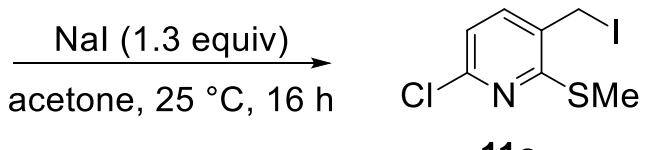

According to literature ${ }^{17}$, to 3-bromo-6-chloro-2-(methylthio)pyridine (3.22 g, $13.5 \mathrm{mmol}$, 1.0 equiv) in THF (14 mL) was added $i \operatorname{PrMgCl} \cdot \mathrm{LiCl}(1.22 \mathrm{M}$ in THF, $12.2 \mathrm{~mL}, 14.9 \mathrm{mmol}, 1.1$ equiv) at $-30{ }^{\circ} \mathrm{C}$. The mixture was stirred at $-30{ }^{\circ} \mathrm{C}$ for $2 \mathrm{~h}$. A solution of $N, N, N^{\prime}, N^{\prime}$-tetramethyl methylene diamine $\left(2.02 \mathrm{~mL}, 14.9 \mathrm{mmol}, 1.1\right.$ equiv) in DCM $(15 \mathrm{~mL})$ was prepared at $0{ }^{\circ} \mathrm{C}$. Trifluoroacetic anhydride $\left(2.09 \mathrm{~mL}, 14.9 \mathrm{mmol}, 1.1\right.$ equiv) was carefully added at $0{ }^{\circ} \mathrm{C}$ and the

\footnotetext{
${ }^{17}$ Barl, N. M.; Sansiaume-Dagousset, E.; Monzón, G.; Wagner, A. J.; Knochel, P. Org. Lett. 2014, $16,2422$.
} 
mixture was stirred for $30 \mathrm{~min}$. The methylene(dimethyl)iminium trifluoroacetate solution was added dropwise at $0{ }^{\circ} \mathrm{C}$ to the prepared Grignard reagent and stirred for $2 \mathrm{~h}$ at $25{ }^{\circ} \mathrm{C}$. The mixture was quenched with sat. aq. $\mathrm{NaHCO}_{3}$ solution $(30 \mathrm{~mL})$. The aqueous phase was extracted with EtOAc $(3 \times 30 \mathrm{~mL})$ and the combined organic phases were dried over anhydrous $\mathrm{Na}_{2} \mathrm{SO}_{4}$ and filtrated. After removal of solvent, flash chromatographical purification (silica gel, DCM:EtOH = 95:5) afforded the title compound (2.81 g, $13.0 \mathrm{mmol}, 96 \%$ yield).

${ }^{1} \mathrm{H}-\mathrm{NMR}(400 \mathrm{MHz}, \mathbf{C D C l} 3): \delta / \mathrm{ppm}=7.46(\mathrm{~d}, J=7.8 \mathrm{~Hz}, 1 \mathrm{H}), 6.98(\mathrm{~d}, J=7.8 \mathrm{~Hz}, 1 \mathrm{H})$, $3.35(\mathrm{~s}, 2 \mathrm{H}), 2.56(\mathrm{~s}, 3 \mathrm{H}), 2.26(\mathrm{~s}, 6 \mathrm{H})$.

${ }^{13}$ C-NMR (100 MHz, $\left.\mathbf{C D C l} 3\right): \delta / p p m=159.9,149.2,138.6,130.8,118.8,59.7,45.6$ (2C), 13.6.

According to literature ${ }^{18}$, to a solution of 6-chloro-3-(chloromethyl)-2-(methylthio)pyridine (2.81 g, $13.0 \mathrm{mmol}, 1.0$ equiv) in $\mathrm{CHCl}_{3}(13 \mathrm{~mL})$ was added ethyl chloroformate $(1.36 \mathrm{~mL}$, $14.3 \mathrm{mmol}, 1.1$ equiv) at $0{ }^{\circ} \mathrm{C}$. The mixture was allowed to slowly warm up to $25^{\circ} \mathrm{C}$ and stirred for $16 \mathrm{~h}$. The mixture was quenched with $\mathrm{H}_{2} \mathrm{O}$ and the aqueous layer was extracted with EtOAc $(3 \times 30 \mathrm{~mL})$. The combined organic layers were dried over anhydrous $\mathrm{Na}_{2} \mathrm{SO}_{4}$ and filtrated, solvents were removed in vacuo and the crude product was purified by flash column chromatography (isohexane $\rightarrow$ isohexane:EtOAc 9:1). The title compound was obtained as a colorless amorphous solid (1.91 g, $9.2 \mathrm{mmol}, 71 \%$ yield).

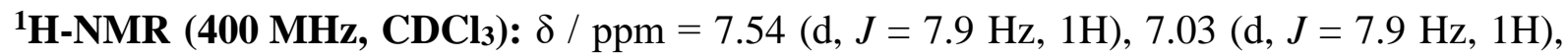
4.57 (s, 2H), 2.62 (s, 3H).

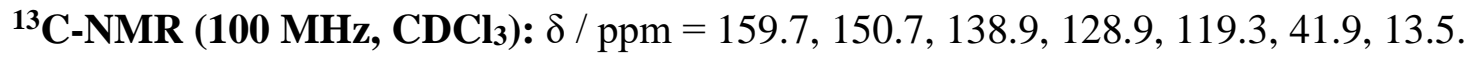

6-Chloro-3-(chloromethyl)-2-(methylthio)pyridine $(2.08 \mathrm{~g}, 10.0 \mathrm{mmol}, 1.0$ equiv) was dissolved in dry acetone $(120 \mathrm{~mL})$ and $\mathrm{NaI}(2.18 \mathrm{~g}, 13.0 \mathrm{mmol}, 1.3$ equiv) was added in a flame dried flask covered in aluminium foil. The mixture was stirred for $16 \mathrm{~h}$ at $25{ }^{\circ} \mathrm{C}$. Solvents were evaporated in vacuo. The crude residues were transferred with $\mathrm{Et}_{2} \mathrm{O}(30 \mathrm{~mL})$ into a separatory funnel and washed with sat. $a q . \mathrm{Na}_{2} \mathrm{~S}_{2} \mathrm{O}_{3}(30 \mathrm{~mL})$. The aqueous layer was extracted with $\mathrm{Et}_{2} \mathrm{O}$ $(3 \times 30 \mathrm{~mL})$. The combined organic layers were dried over $\mathrm{MgSO}_{4}$. After filtration, a copper turning was added to the filtrate and the solvents were removed in vacuo to obtain the desired product as slightly yellow crystals $(2.10 \mathrm{~g}, 7.0 \mathrm{mmol}, 70 \%$ yield $)$, which was stored at $-24{ }^{\circ} \mathrm{C}$ together with a copper turning.

\footnotetext{
${ }^{18}$ Barl, N. M.; Sansiaume-Dagousset, E.; Monzón, G.; Wagner, A. J.; Knochel, P. Org. Lett. 2014, 16, 2422.
} 
${ }^{1}$ H-NMR (400 MHz, CDCl3): $\delta$ / ppm = $7.46(\mathrm{~d}, J=7.9,1 \mathrm{H}), 6.97(\mathrm{~d}, J=7.9,1 \mathrm{H}), 4.38(\mathrm{~s}, 2 \mathrm{H})$, $2.62(\mathrm{~s}, 3 \mathrm{H})$.

${ }^{13}$ C-NMR (100 MHz, CDCl3): $\delta / \mathrm{ppm}=159.3,150.0,138.7,130.7,119.5,13.6,-0.1$.

MS (EI, 70 eV): $m / z(\%)=174$ (36), 172 (100), 136 (19), 126 (23), 90 (11).

HRMS (EI-orbitrap): m/z: [M] calcd for $\mathrm{C}_{7} \mathrm{H}_{7}$ ClINS 298.9032; Found 298.9024.

m.p. $\left({ }^{\circ} \mathbf{C}\right): 87.5-89.9$. 
NMR data

1,2-Diphenylethan-1-ol (4a)

${ }^{1} \mathrm{H}-\mathrm{NMR}$ (400 MHz, $\mathrm{CDCl}_{3}$ ):

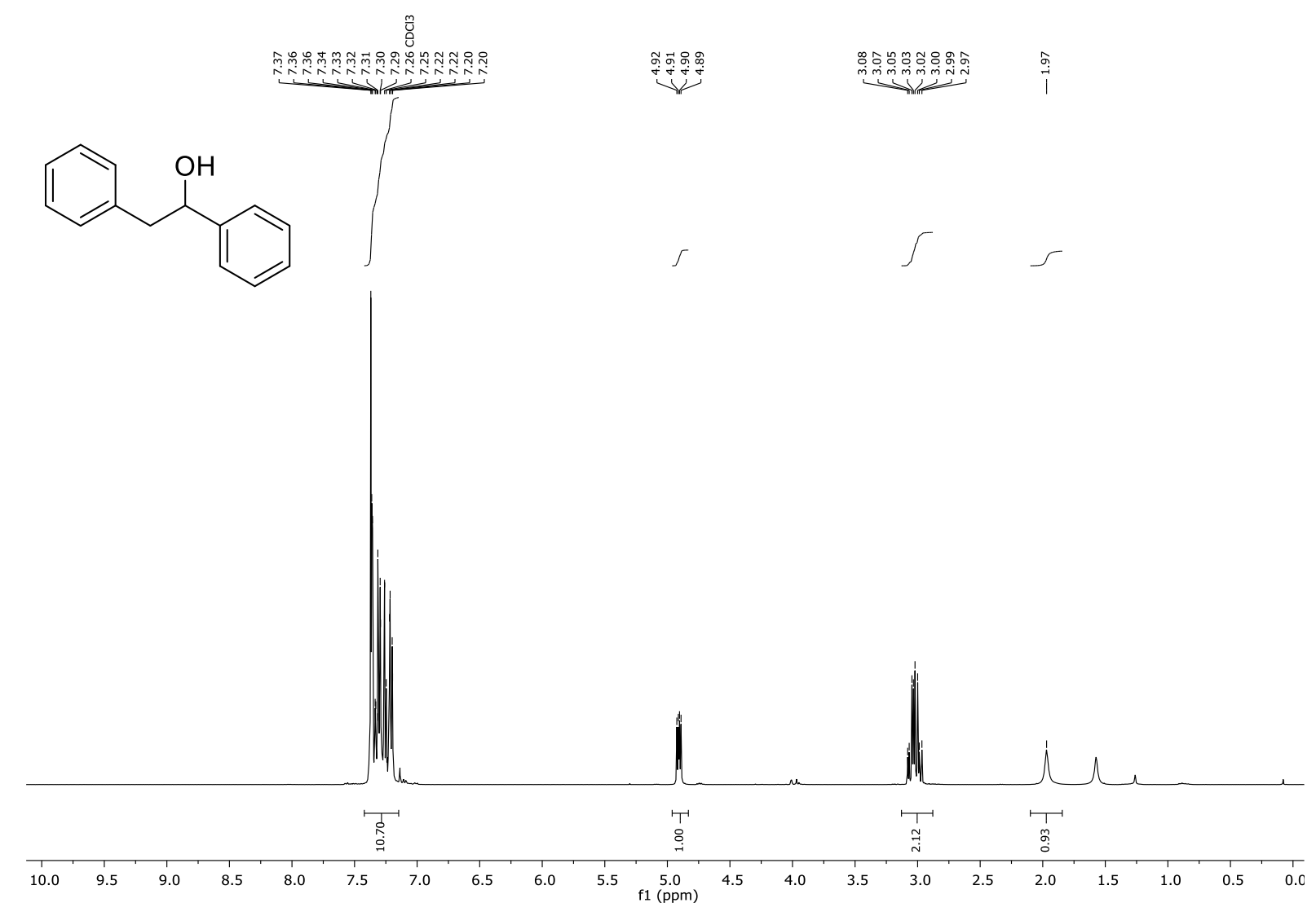

${ }^{13} \mathrm{C}-\mathrm{NMR}$ (100 MHz, $\left.\mathrm{CDCl}_{3}\right)$ :
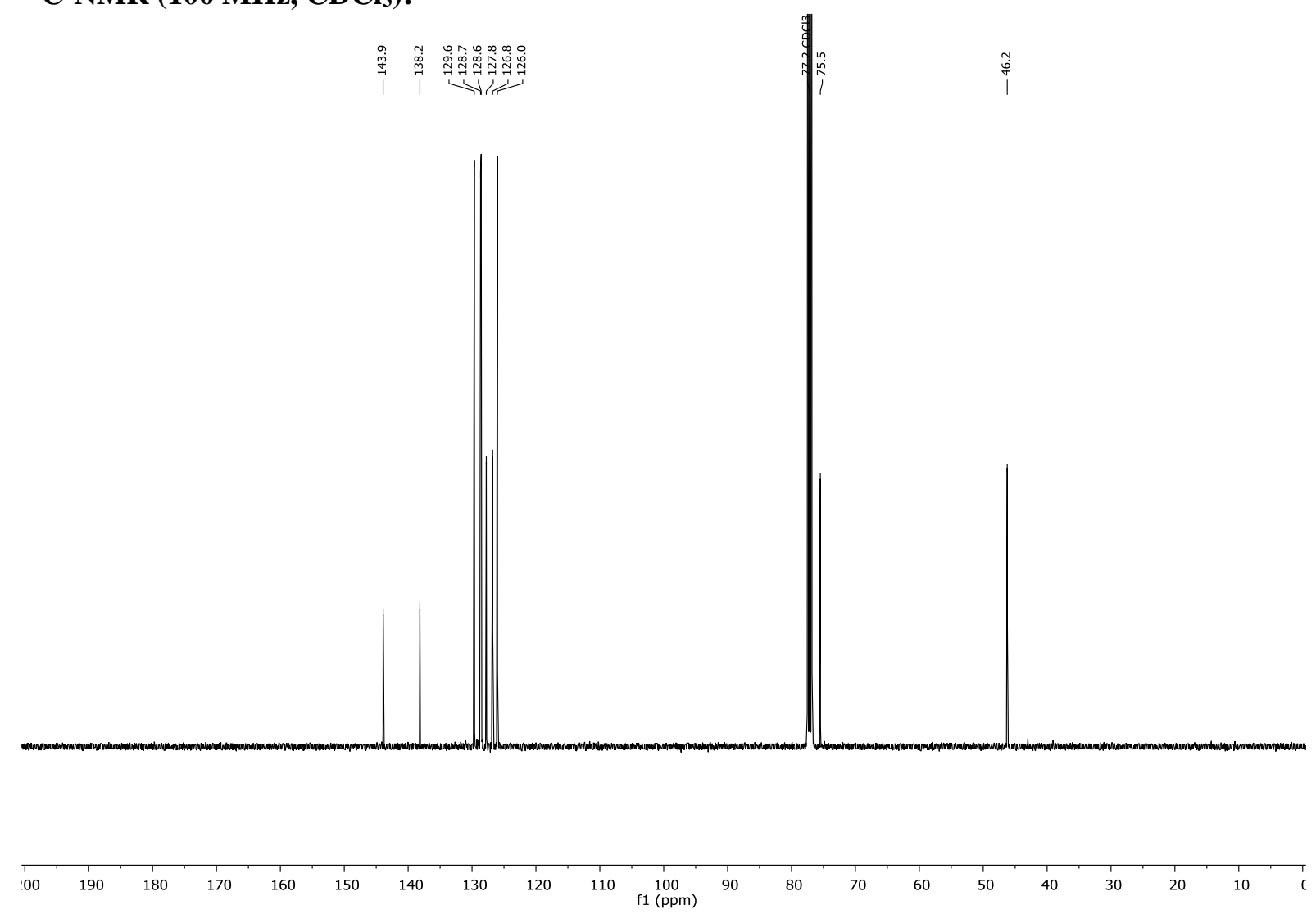

SI-53 
1-(3-Methoxyphenyl)-2-phenylethan-1-ol (4b)

${ }^{1} \mathrm{H}-\mathrm{NMR}$ (600 MHz, $\left.\mathrm{CDCl}_{3}\right)$ :

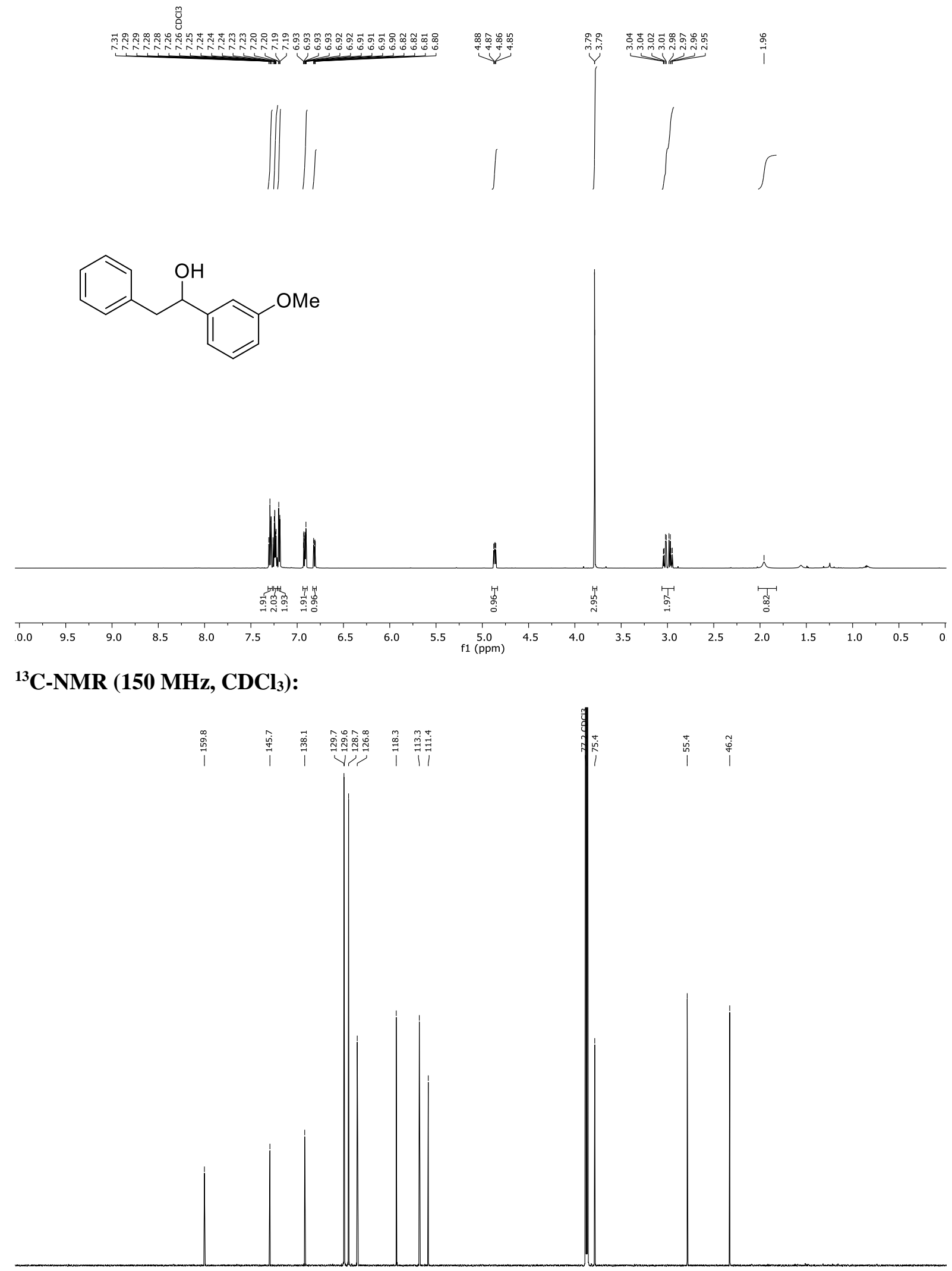

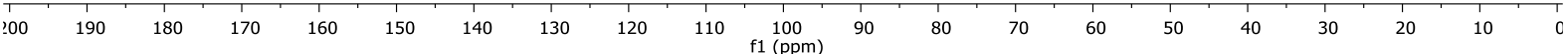


2-(4-(Tert-butyl)phenyl)-1-phenylethan-1-ol (4c)

${ }^{1} \mathrm{H}-\mathrm{NMR}$ (400 MHz, $\mathrm{CDCl}_{3}$ ):

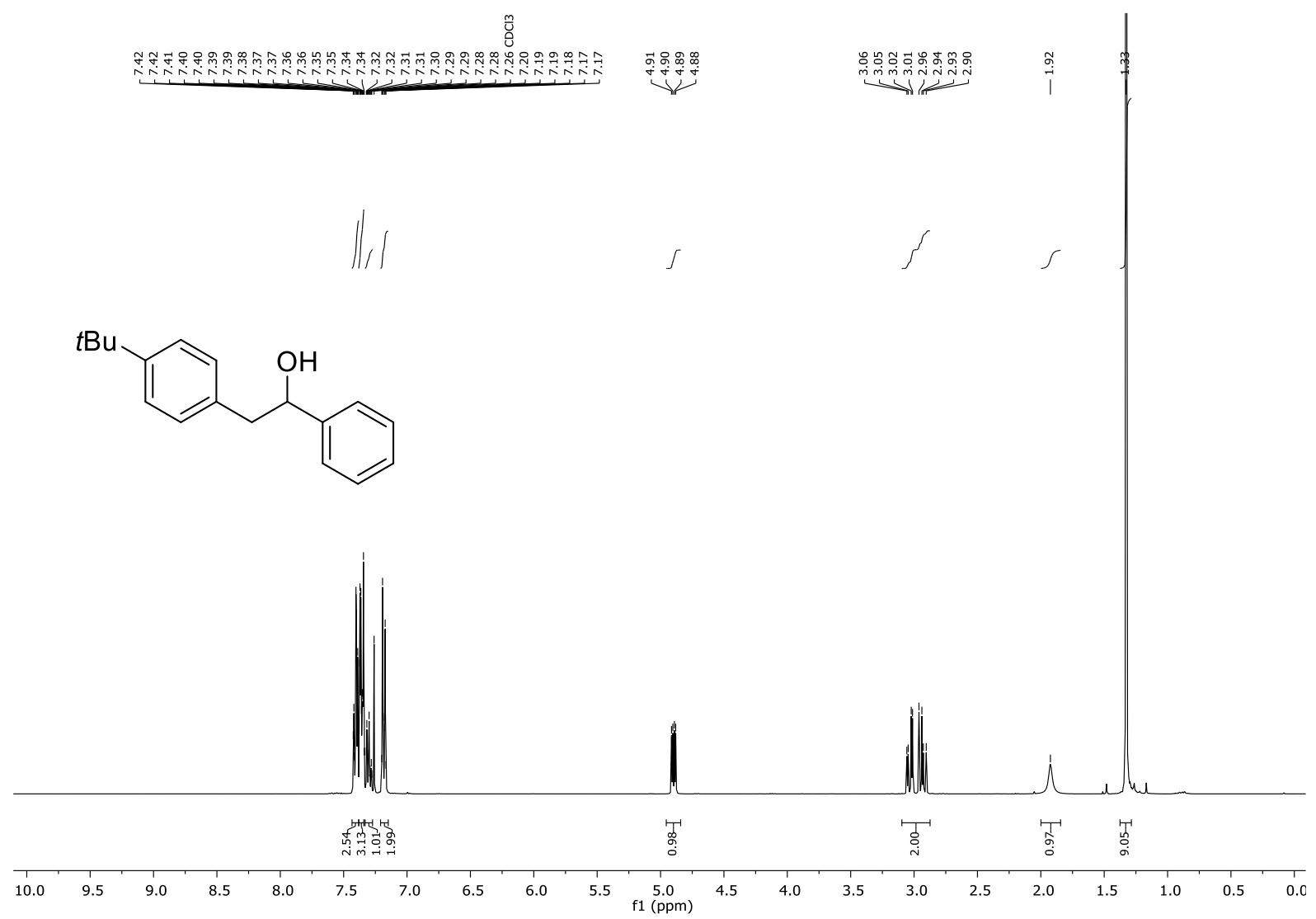

${ }^{13} \mathrm{C}-\mathrm{NMR}\left(100 \mathrm{MHz}, \mathrm{CDCl}_{3}\right)$ :

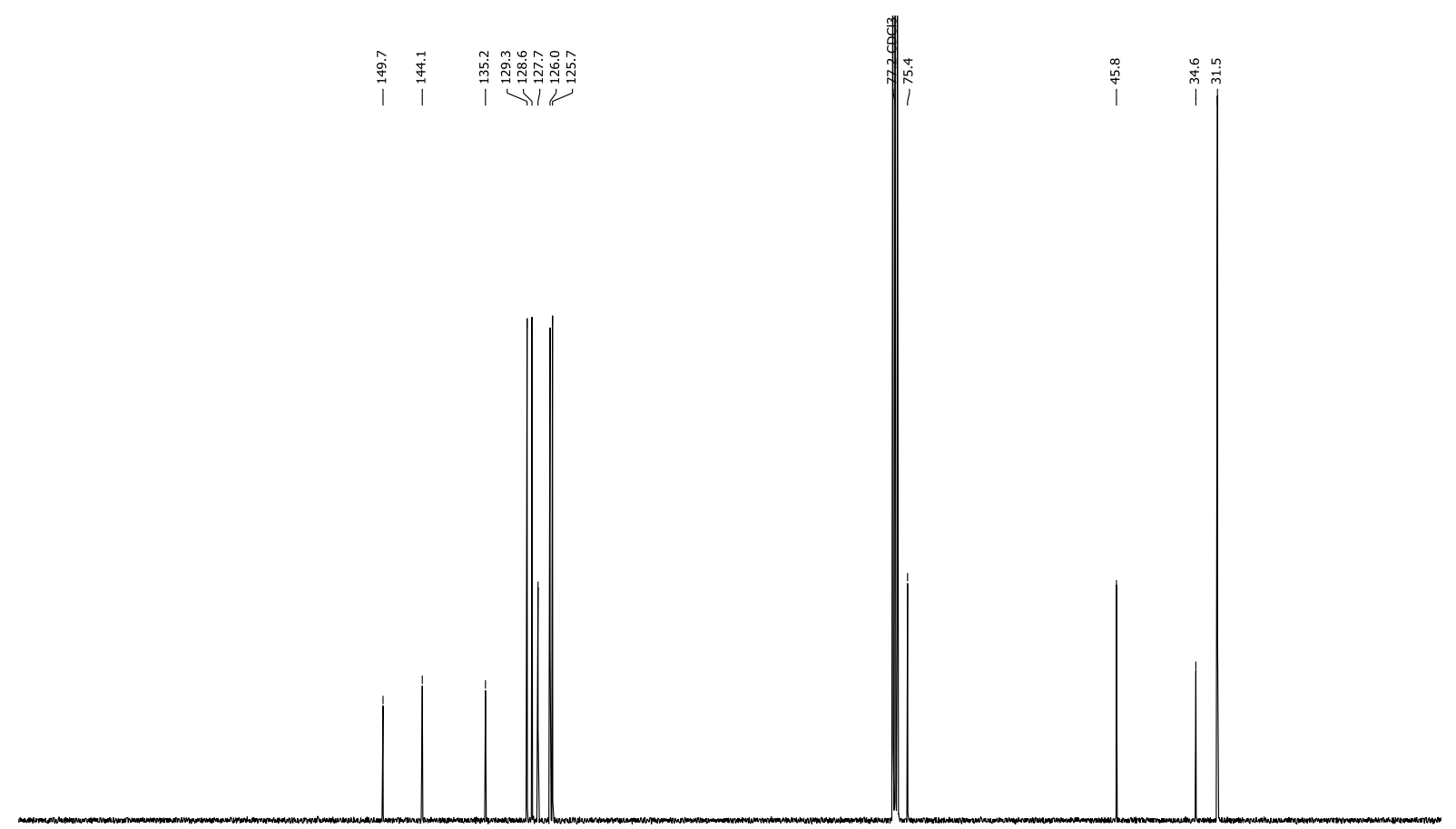


2-(4-(Tert-butyl)phenyl)-1-(4-methoxyphenyl)ethan-1-ol (4d)

${ }^{1} \mathrm{H}-\mathrm{NMR}$ (400 MHz, $\left.\mathrm{CDCl}_{3}\right)$ :

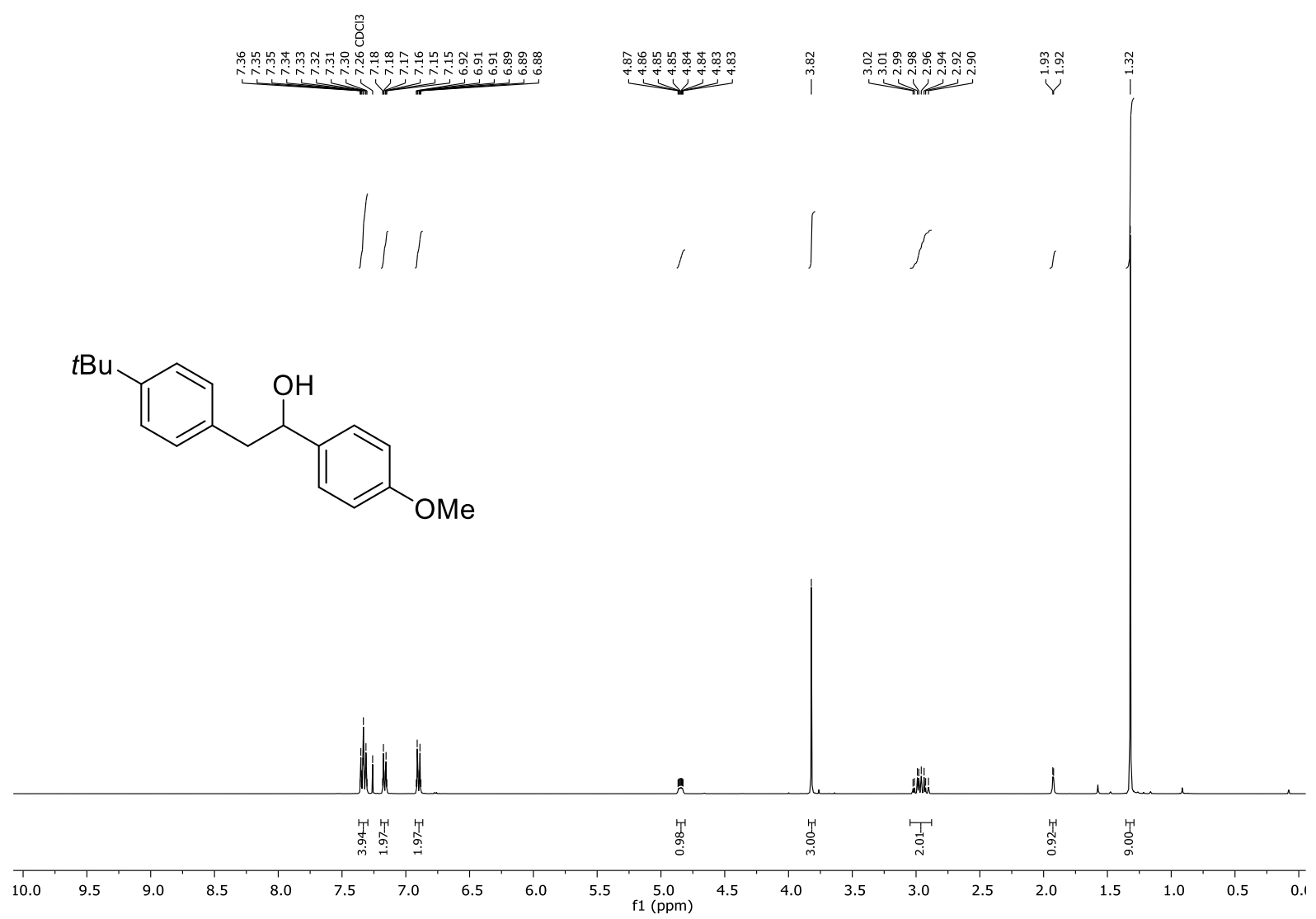

${ }^{13} \mathrm{C}-\mathrm{NMR}\left(100 \mathrm{MHz}, \mathrm{CDCl}_{3}\right)$ :

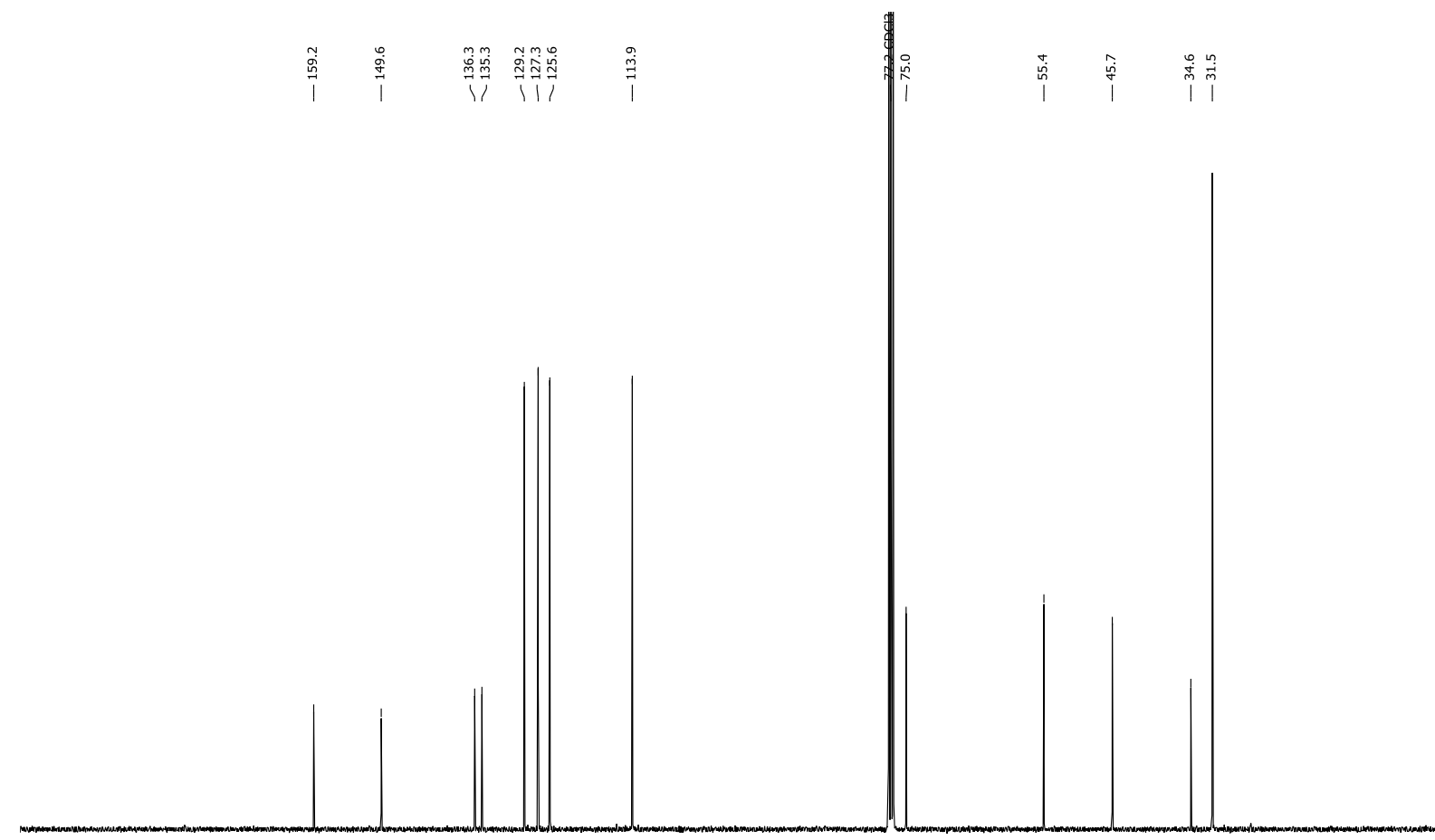


2-(4-Isopropylphenyl)-1-(3-methoxyphenyl)ethan-1-ol (4e)

${ }^{1} \mathrm{H}-\mathrm{NMR}$ (400 MHz, $\left.\mathrm{CDCl}_{3}\right)$ :

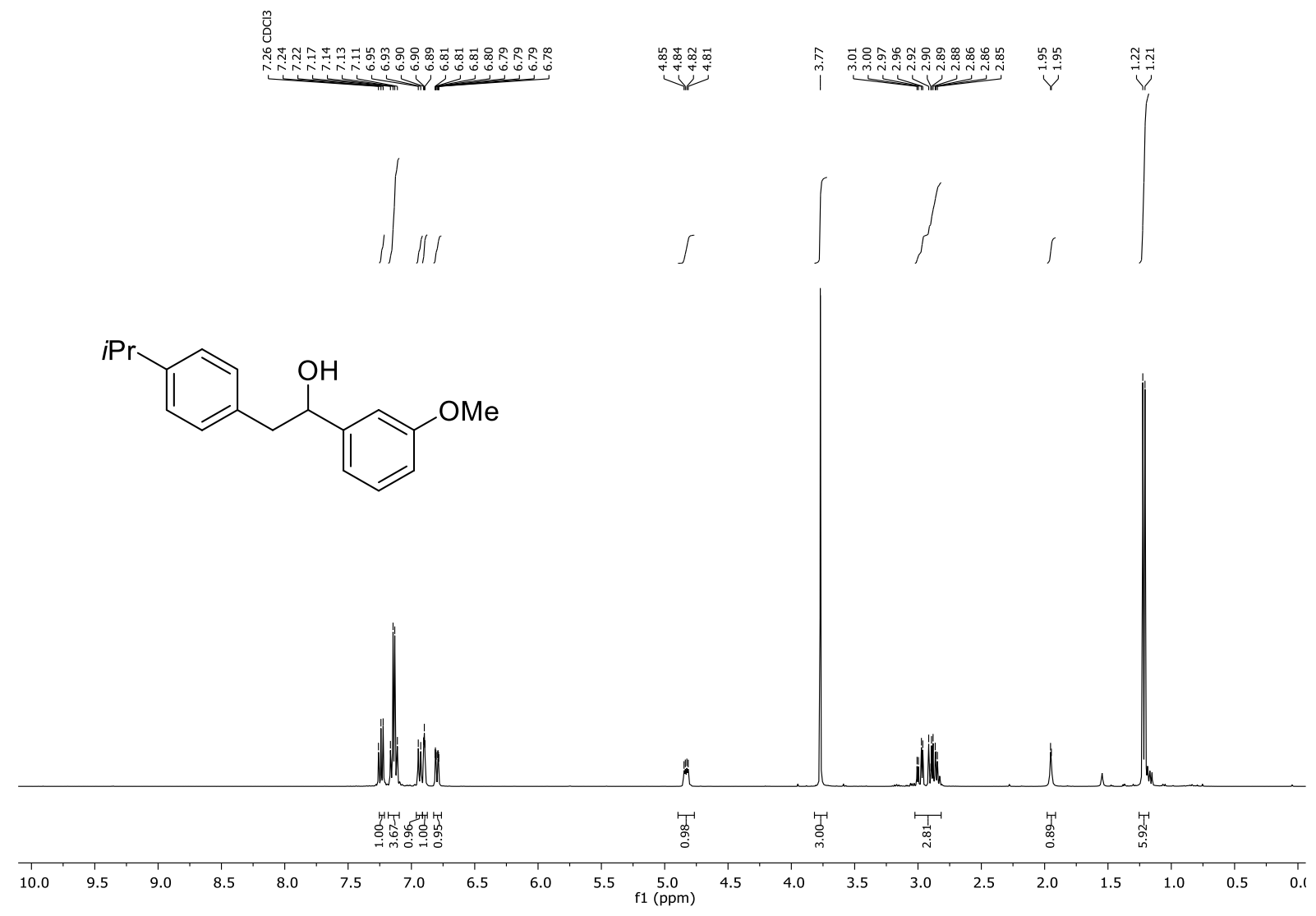

${ }^{13} \mathrm{C}-\mathrm{NMR}\left(100 \mathrm{MHz}, \mathrm{CDCl}_{3}\right)$ :

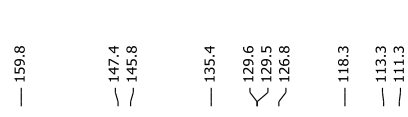
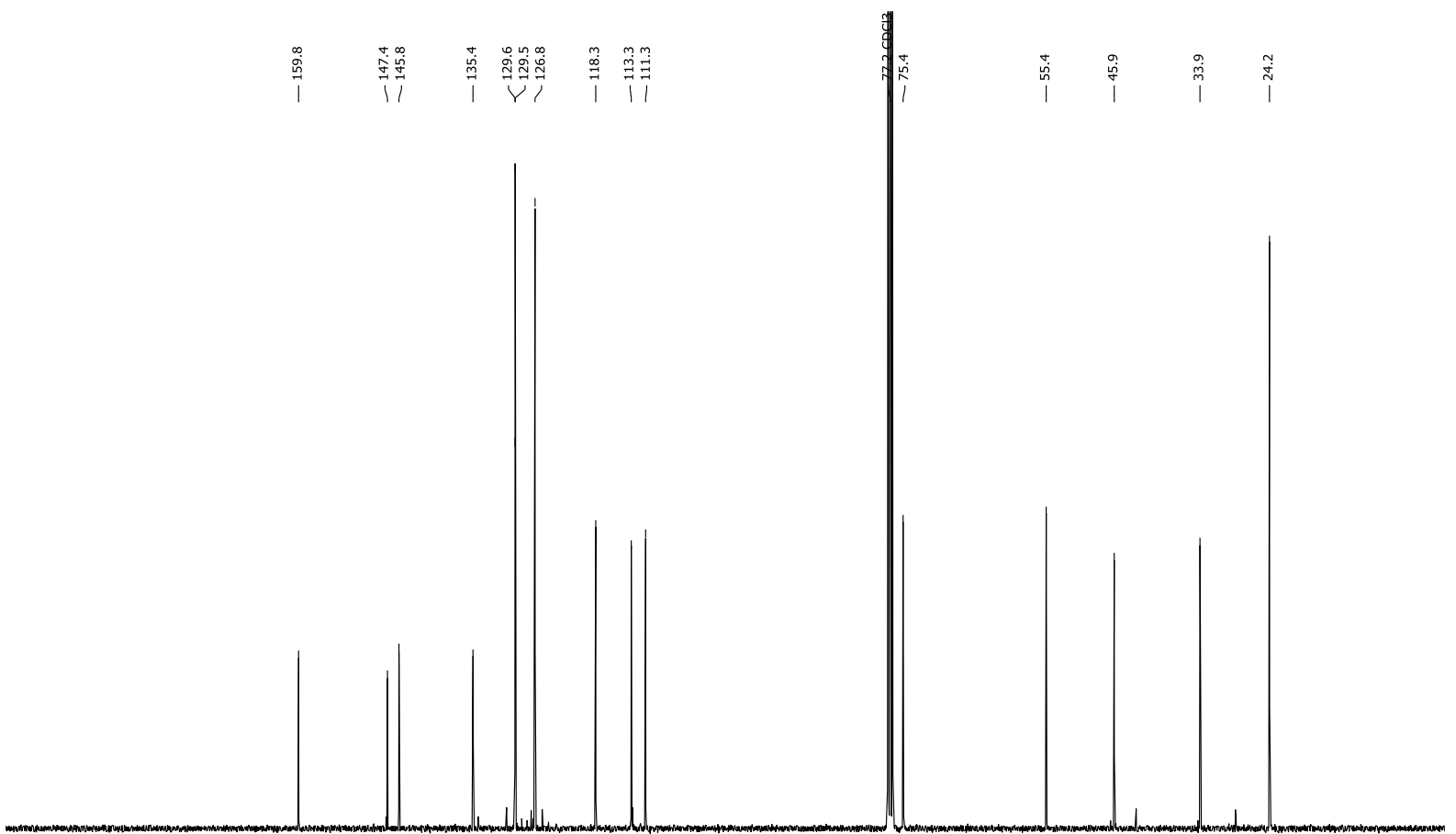

$! 00$

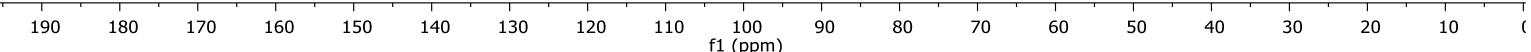


1-Cyclohexyl-2-(4-isopropylphenyl)ethan-1-ol (4f)

${ }^{1} \mathrm{H}-\mathrm{NMR}\left(400 \mathrm{MHz}, \mathrm{CDCl}_{3}\right)$ :

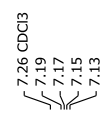

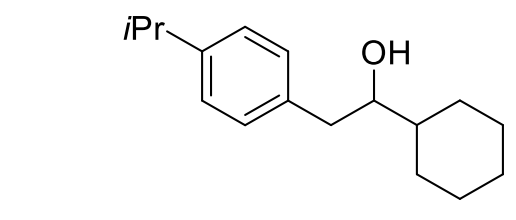

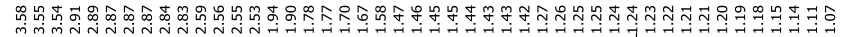
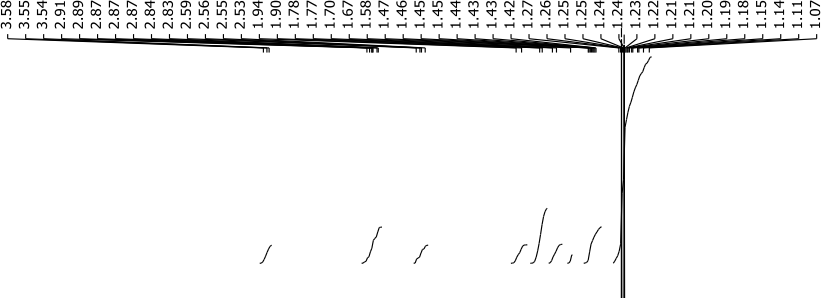

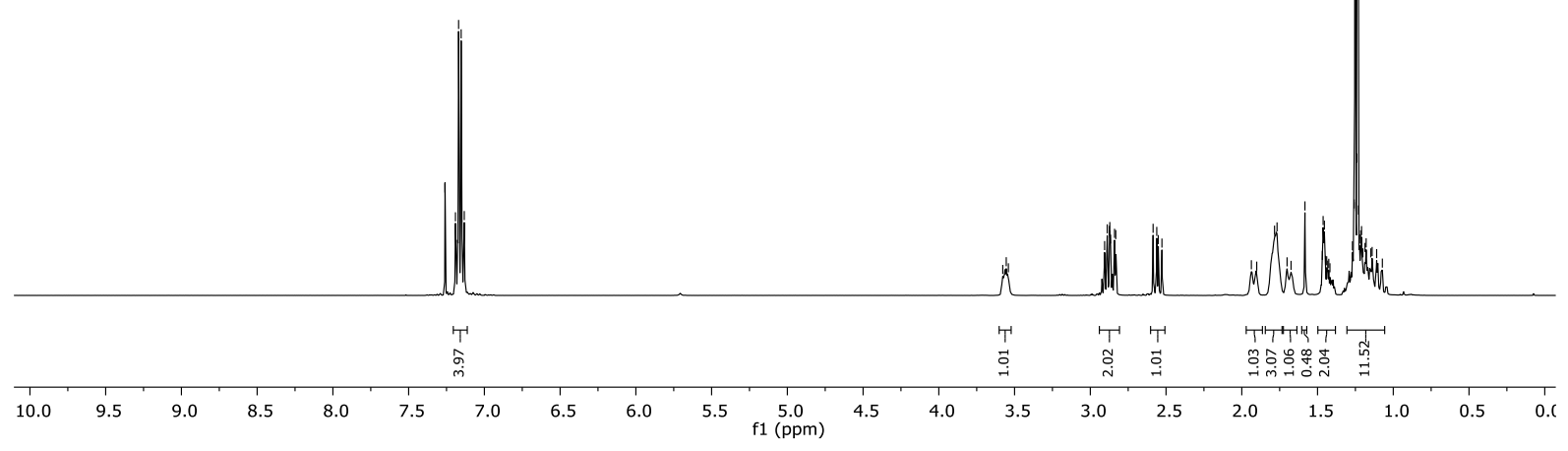

${ }^{13} \mathrm{C}-\mathrm{NMR}\left(100 \mathrm{MHz}, \mathrm{CDCl}_{3}\right)$ :
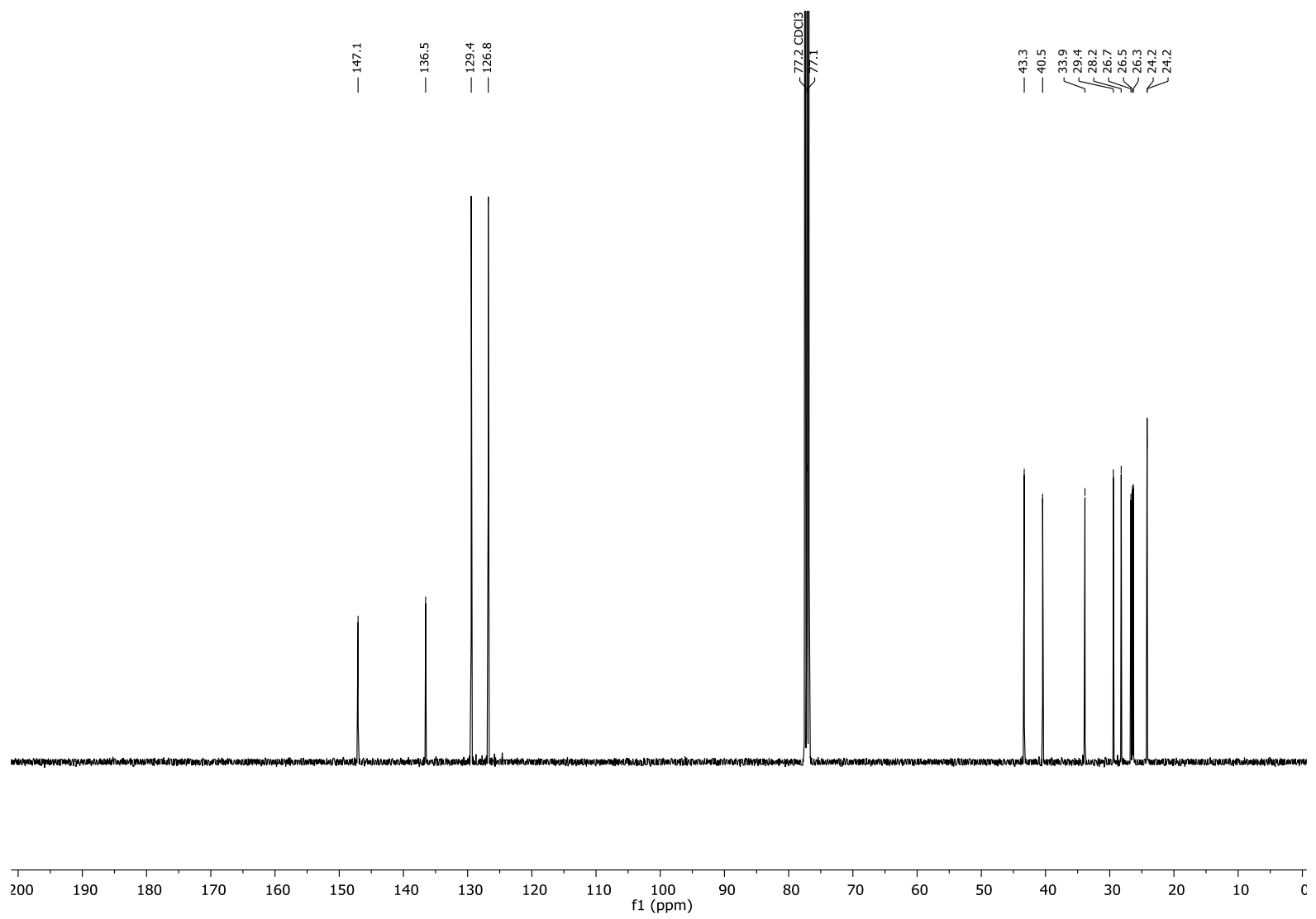

SI-58 
1-(4-Isopropylphenyl)-2-phenylpropan-2-ol (4g)

${ }^{1} \mathrm{H}-\mathrm{NMR}$ (400 MHz, $\mathrm{CDCl}_{3}$ ):

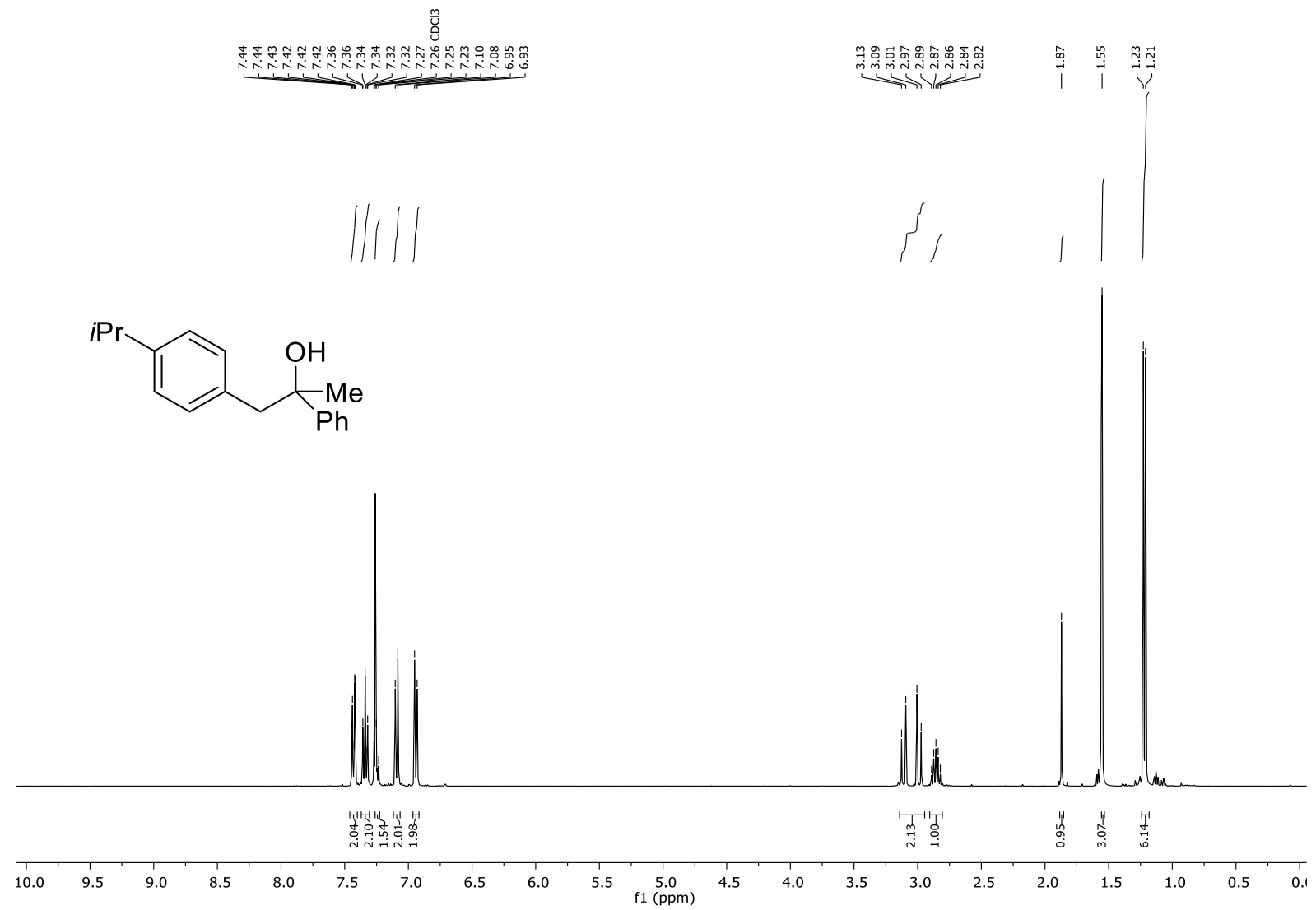

${ }^{13} \mathrm{C}-\mathrm{NMR}\left(100 \mathrm{MHz}, \mathrm{CDCl}_{3}\right)$ :

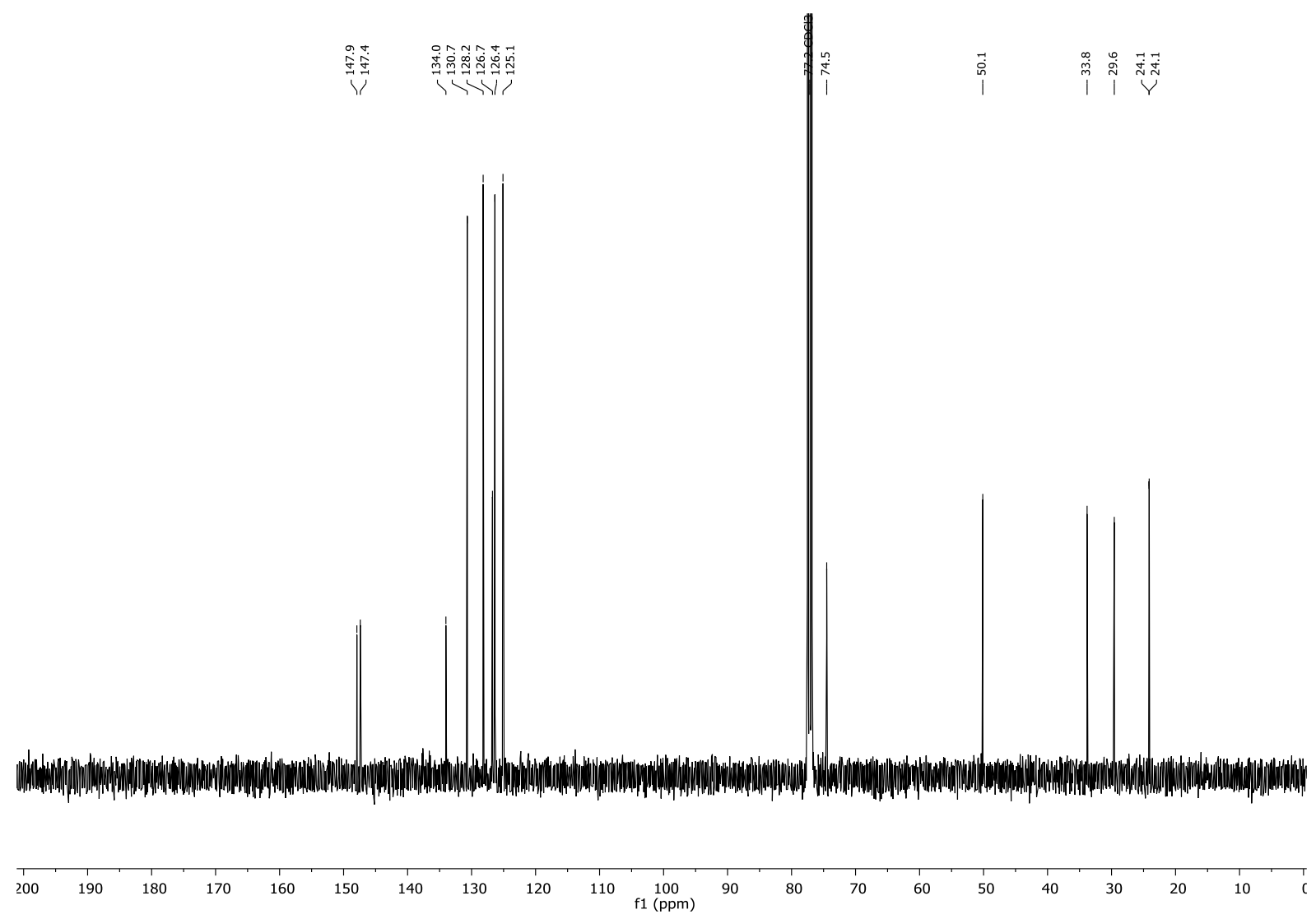


2-(3-((Tert-butyldimethylsilyl)oxy)phenyl)-1-phenylethan-1-ol (7a)

${ }^{1} \mathrm{H}-\mathrm{NMR}$ (400 MHz, $\left.\mathrm{CDCl}_{3}\right)$ :

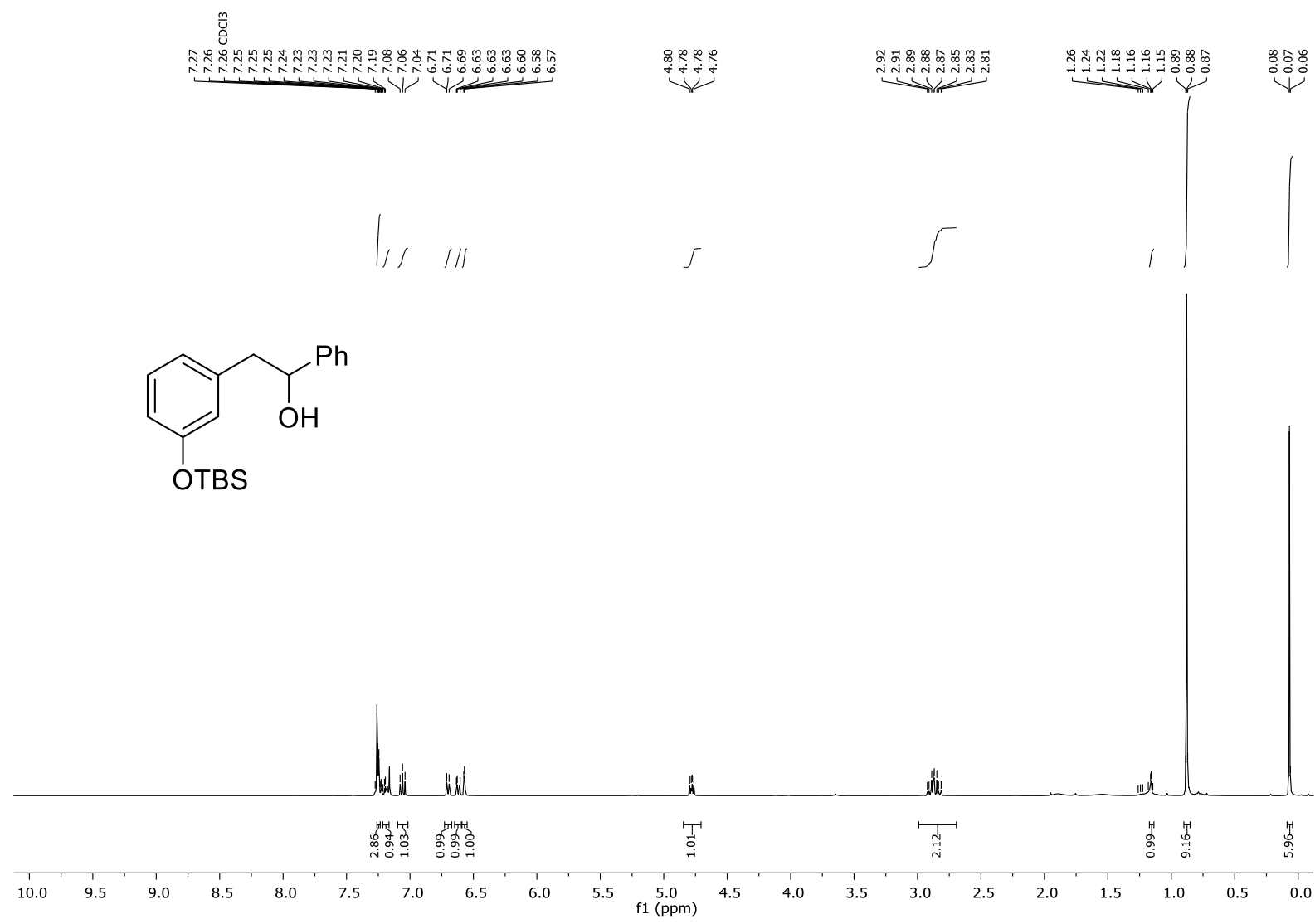

${ }^{13} \mathrm{C}-\mathrm{NMR}\left(100 \mathrm{MHz}, \mathrm{CDCl}_{3}\right)$ :

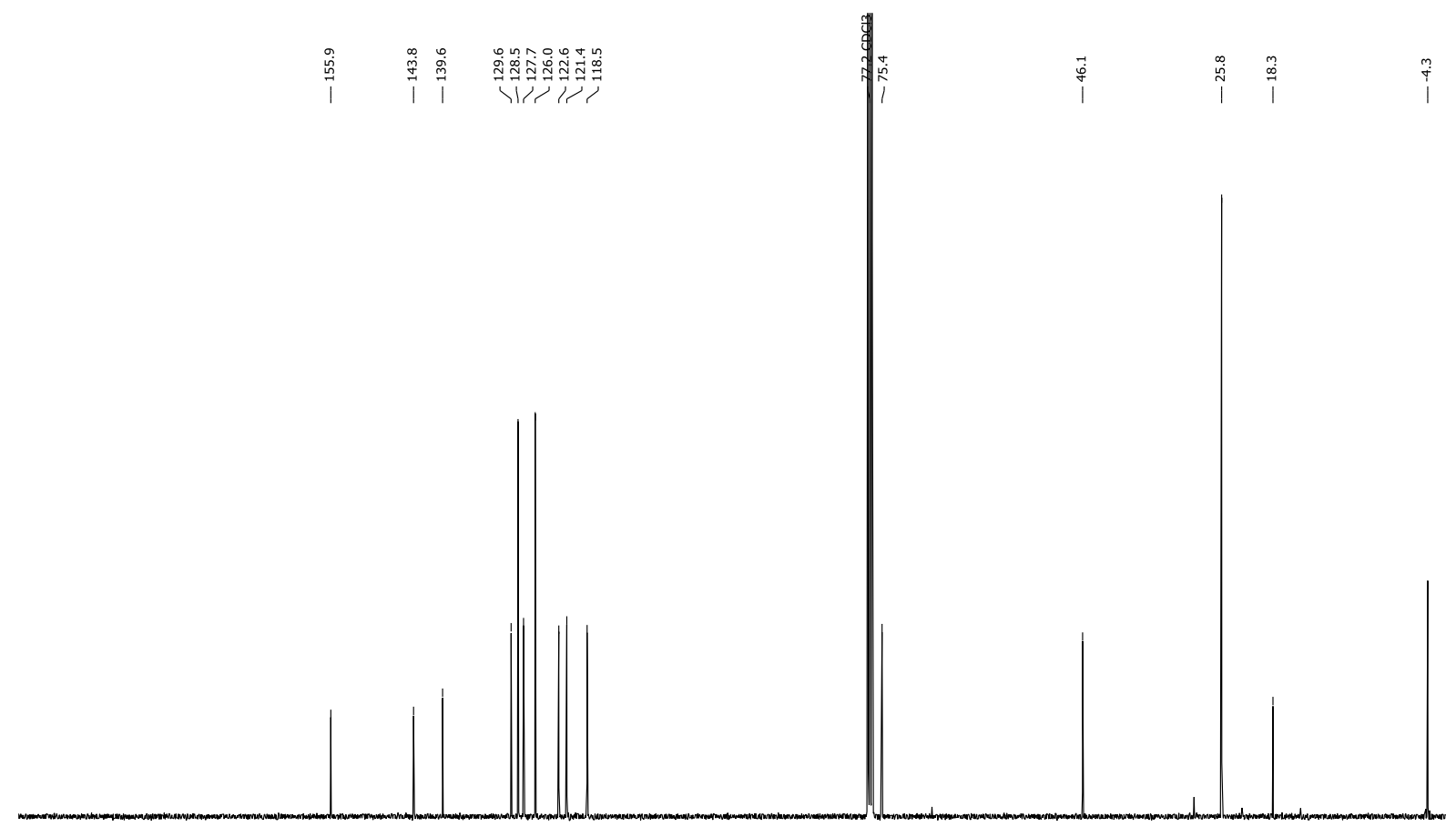

200

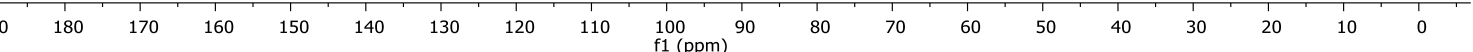


2-(3-((Tert-butyldimethylsilyl)oxy)phenyl)-1-cyclohexylethan-1-ol (7b)

${ }^{1} \mathrm{H}-\mathrm{NMR}$ (400 MHz, $\mathrm{CDCl}_{3}$ ):

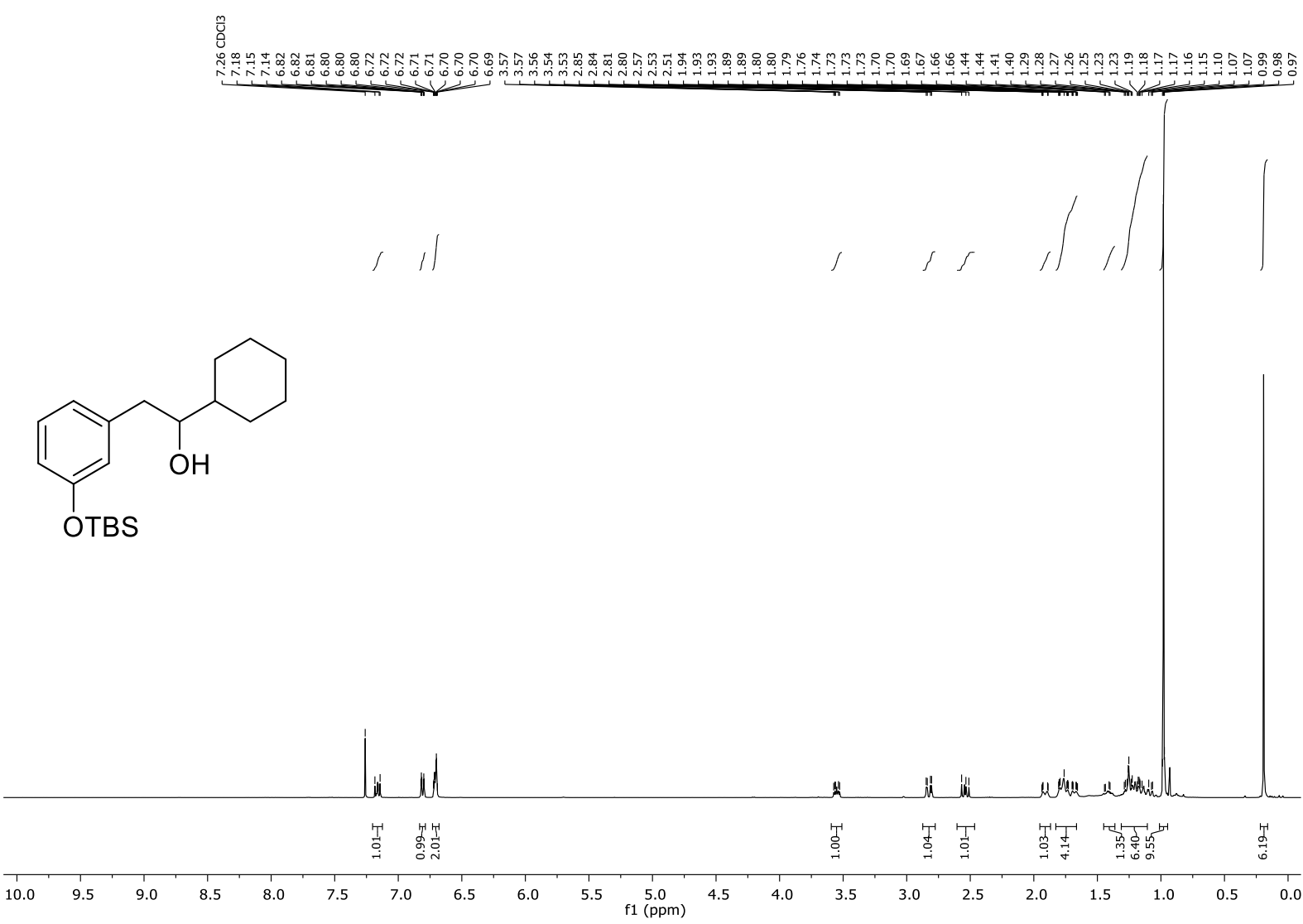

${ }^{13} \mathrm{C}-\mathrm{NMR}\left(100 \mathrm{MHz}, \mathrm{CDCl}_{3}\right)$ :

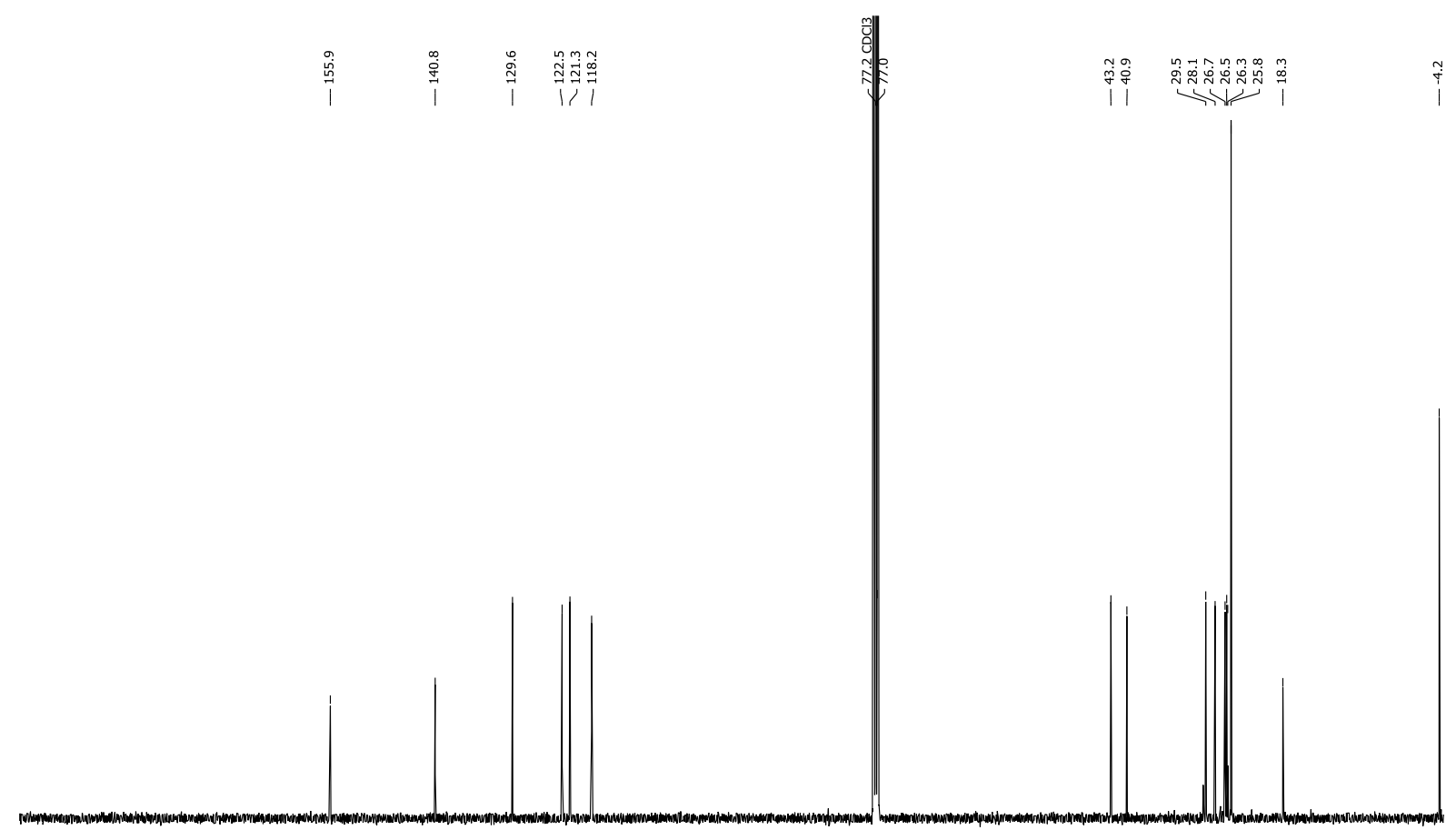


1-Cyclohexyl-2-(4-(methylthio)phenyl)ethan-1-ol (7c)

${ }^{1} \mathrm{H}-\mathrm{NMR}$ (400 MHz, $\mathrm{CDCl}_{3}$ ):

范

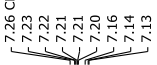<smiles>C#CC=C</smiles><smiles>CSc1ccc(CC(O)C2CCCCC2)cc1</smiles>

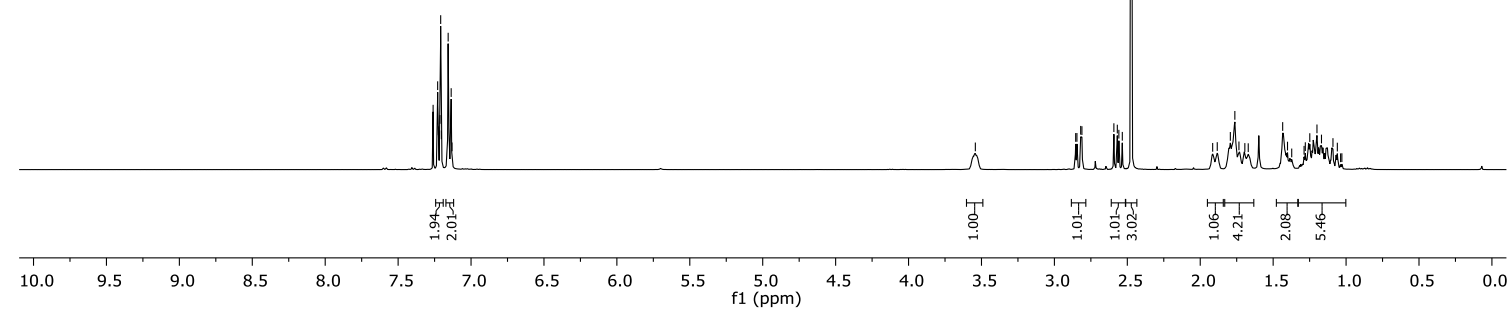

${ }^{13} \mathrm{C}-\mathrm{NMR}$ (100 MHz, $\left.\mathrm{CDCl}_{3}\right)$ :

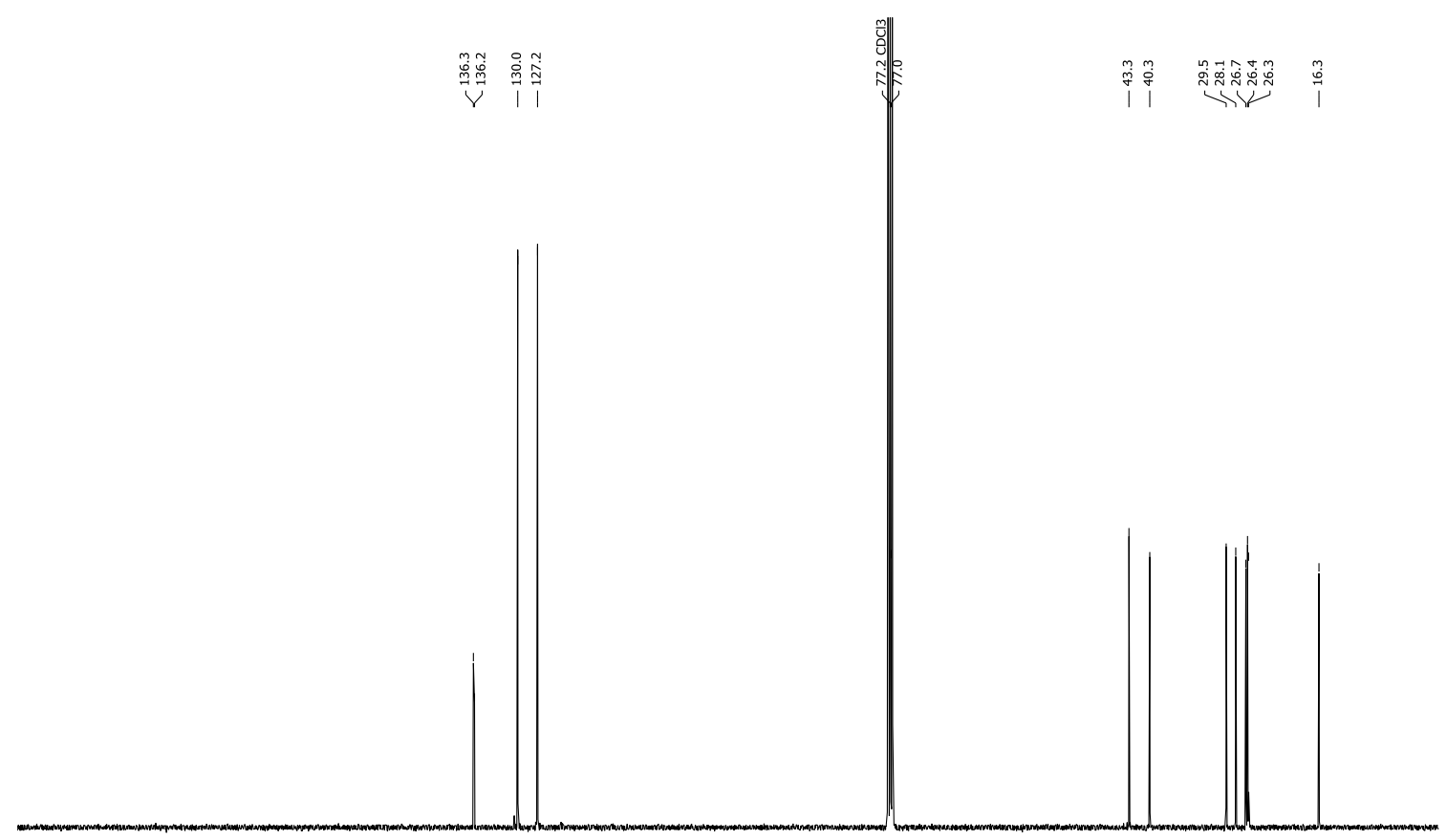

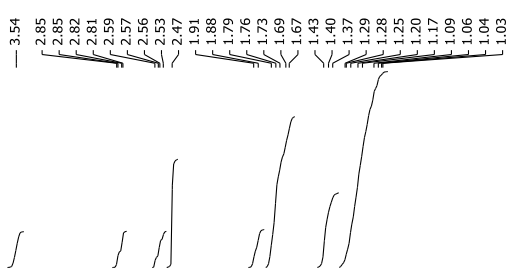

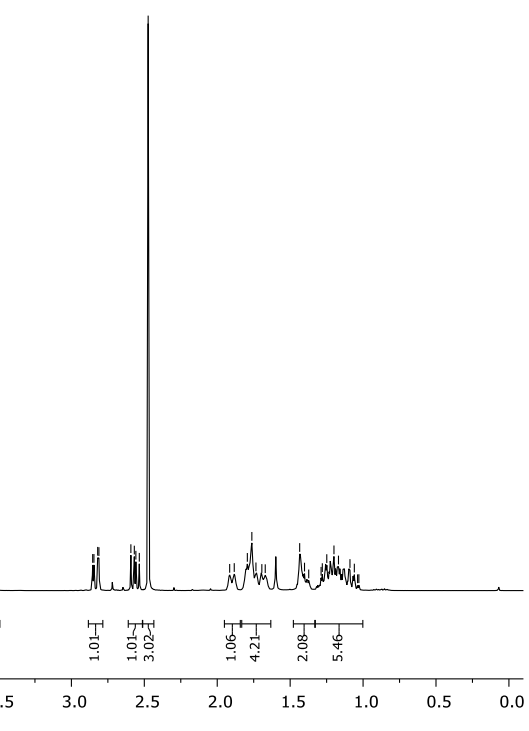


1-(3-Methoxyphenyl)-2-(4-methoxyphenyl)ethan-1-ol (7d)

${ }^{1} \mathrm{H}-\mathrm{NMR}$ (400 MHz, $\mathrm{CDCl}_{3}$ ):

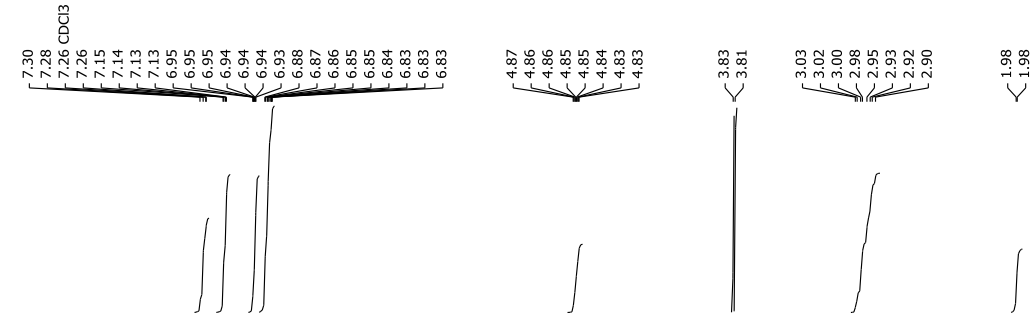

MeOCOMe

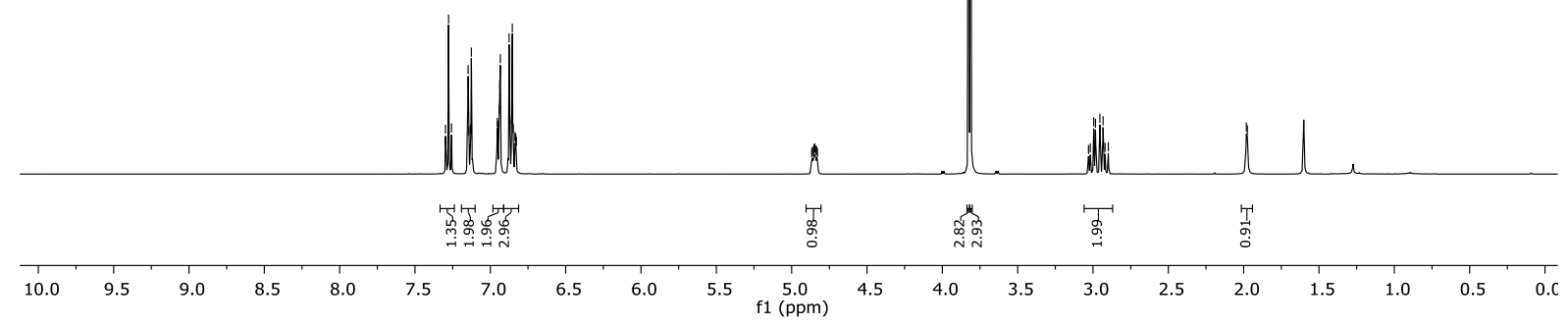

${ }^{13} \mathrm{C}-\mathrm{NMR}$ (100 MHz, $\left.\mathrm{CDCl}_{3}\right)$ :

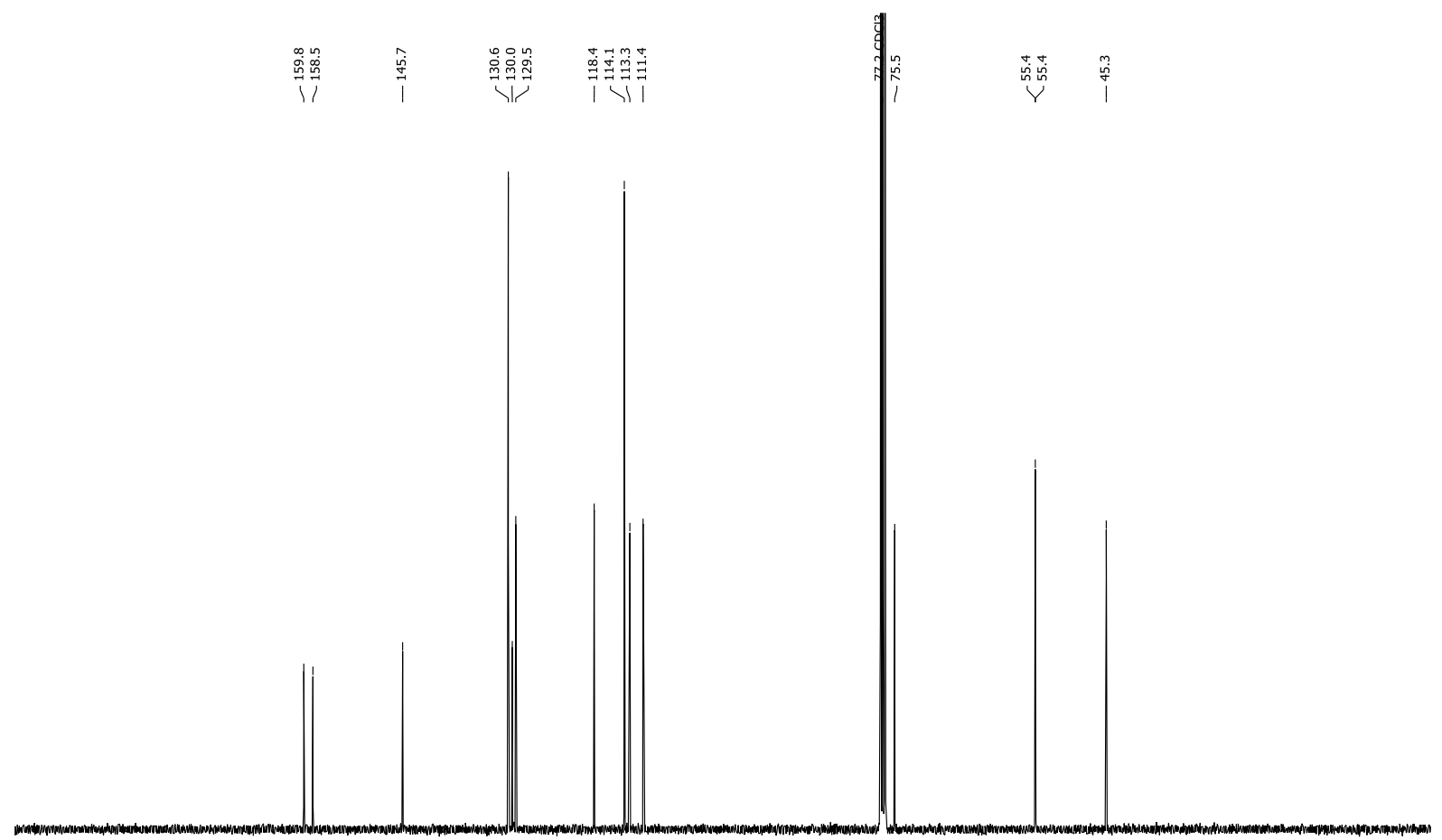

200

$\begin{array}{lllll}190 & 180 & 170 & 160 & 150\end{array}$

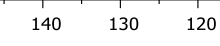

$110 \quad 100$

80

7060

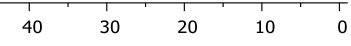




\section{1-(2,4-Dimethylphenyl)-2-(3-methoxyphenyl)ethan-1-ol (7e)}

${ }^{1} \mathrm{H}-\mathrm{NMR}$ (400 MHz, $\left.\mathrm{CDCl}_{3}\right)$ :

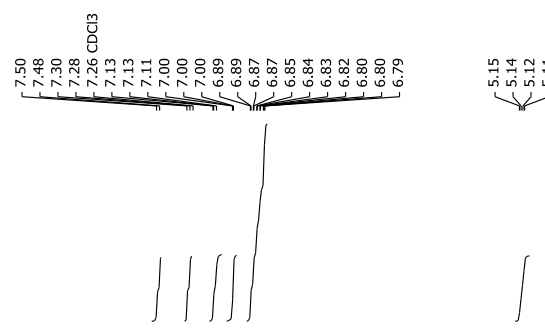<smiles>COc1cccc(CC(O)c2ccc(C)cc2C)c1</smiles>

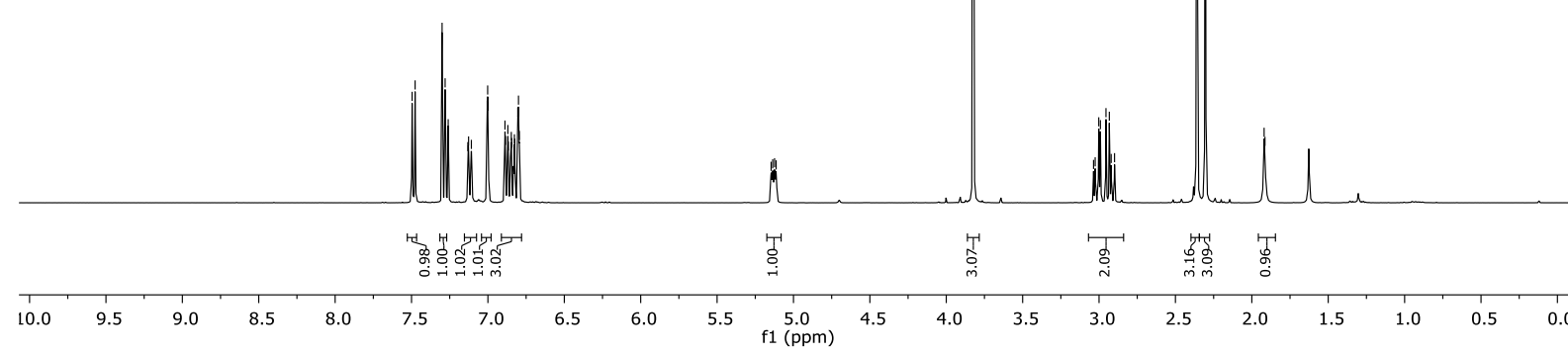

${ }^{13} \mathrm{C}-\mathrm{NMR}\left(100 \mathrm{MHz}, \mathrm{CDCl}_{3}\right)$ :

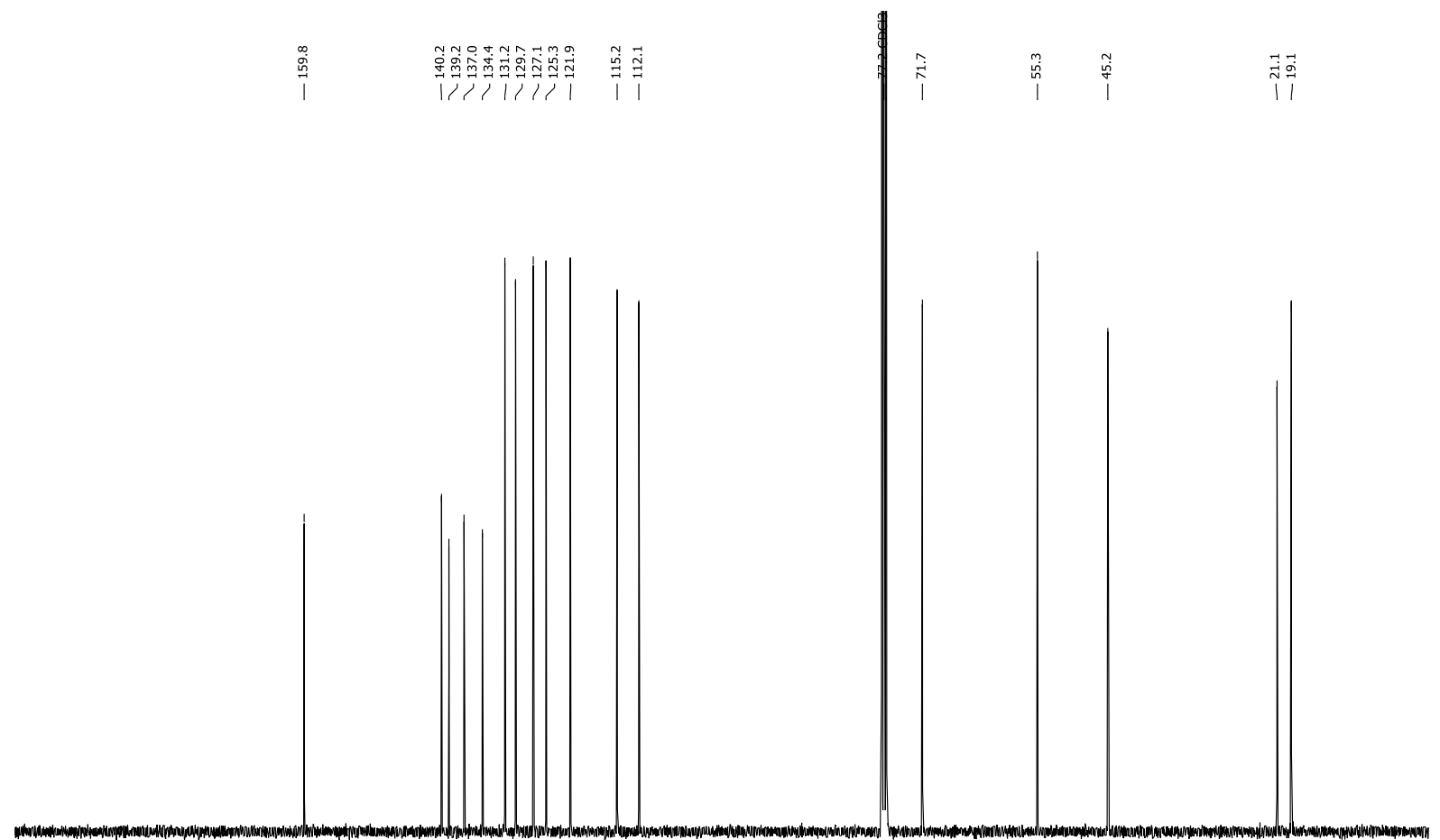


2-(3-Methoxybenzyl)bicyclo[2.2.1]heptan-2-ol (7f)

${ }^{1} \mathrm{H}-\mathrm{NMR}$ (400 MHz, $\mathrm{CDCl}_{3}$ ):

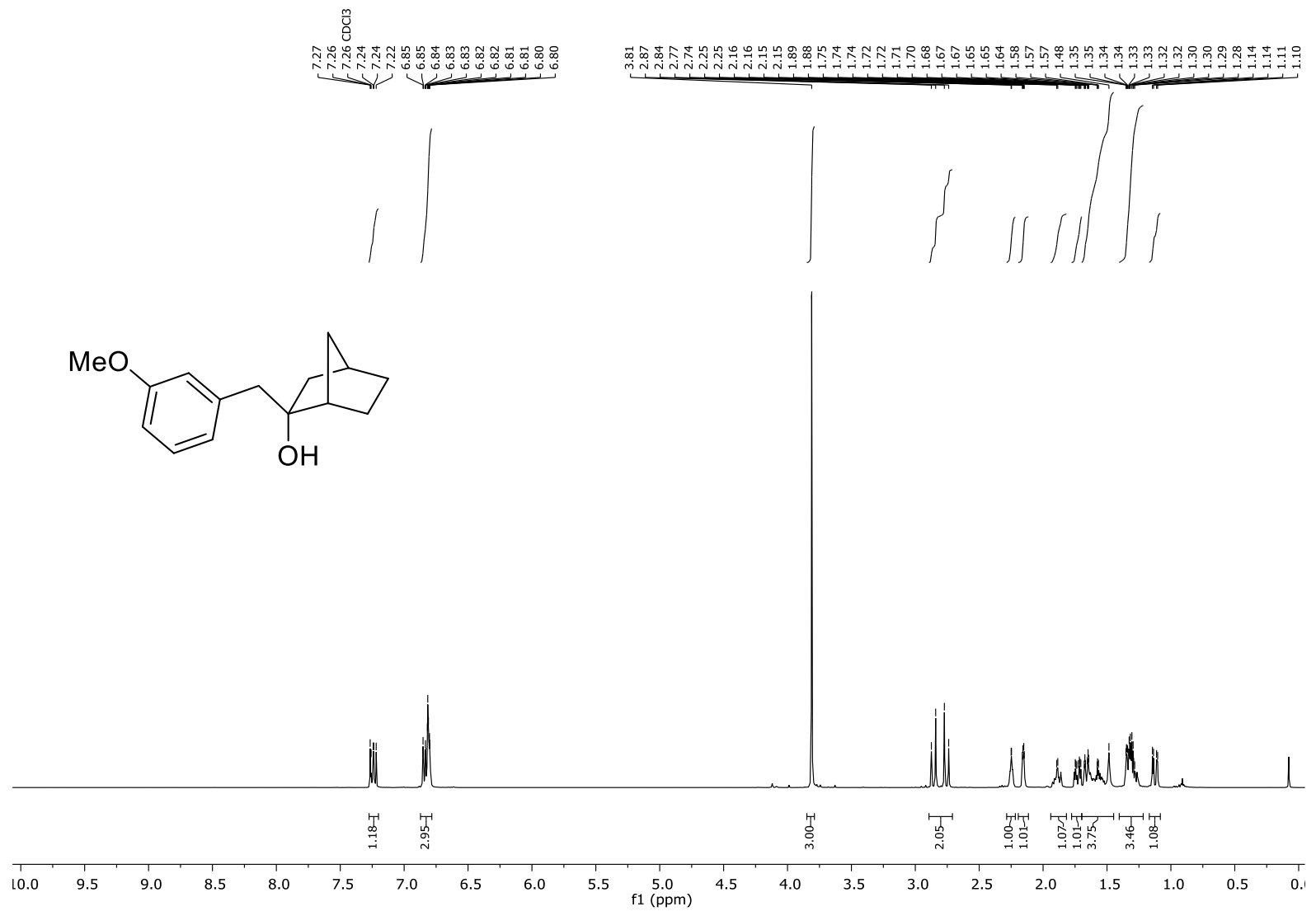

${ }^{13} \mathrm{C}-\mathrm{NMR}\left(100 \mathrm{MHz}, \mathrm{CDCl}_{3}\right)$ :

$$
\text { 尊 }
$$

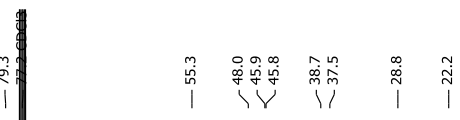




\section{2-(3-Methoxybenzyl)adamantan-2-ol (7g)}

${ }^{1} \mathrm{H}-\mathrm{NMR}$ (400 MHz, $\mathrm{CDCl}_{3}$ ):

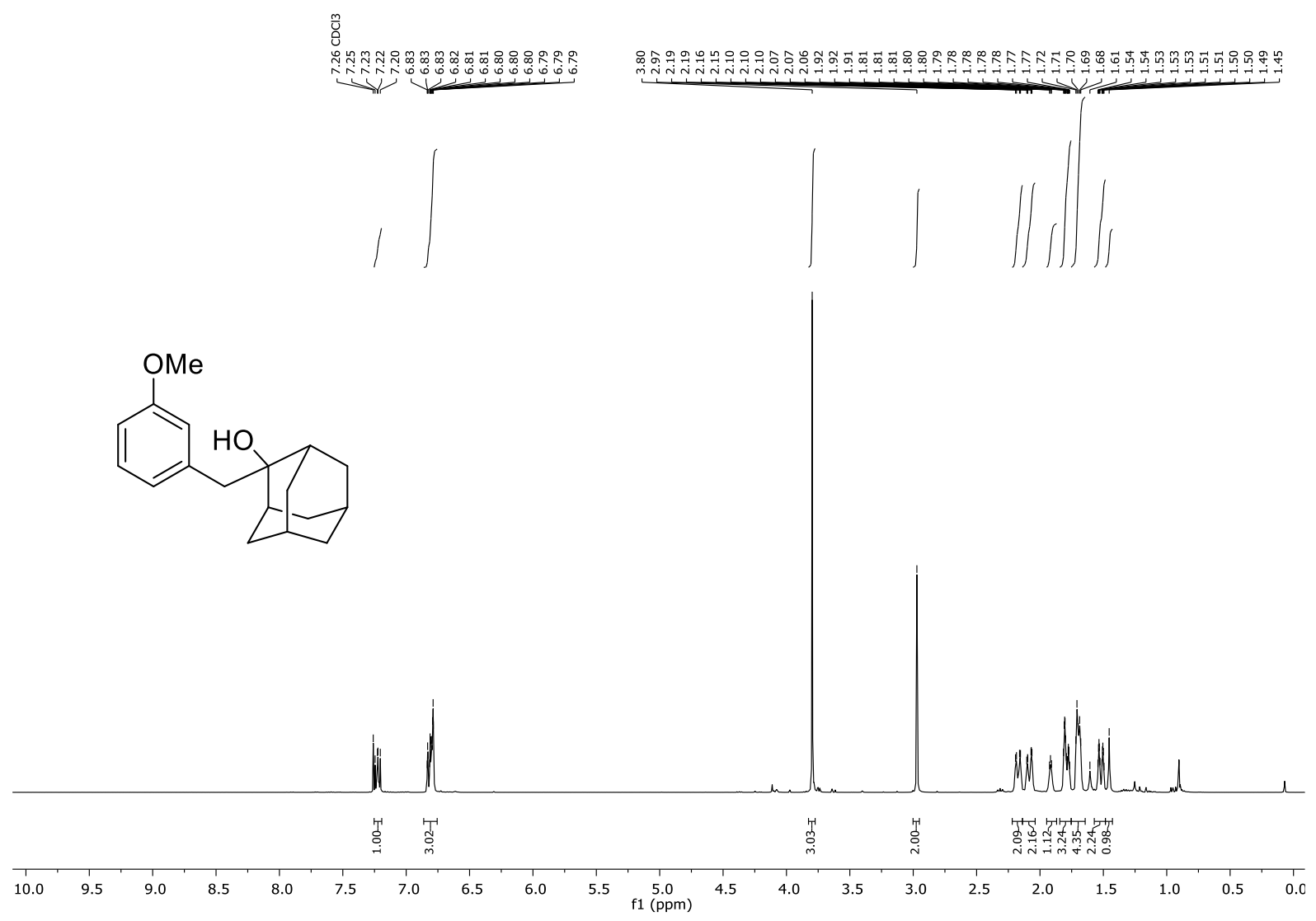

${ }^{13} \mathrm{C}-\mathrm{NMR}\left(100 \mathrm{MHz}, \mathrm{CDCl}_{3}\right)$ :

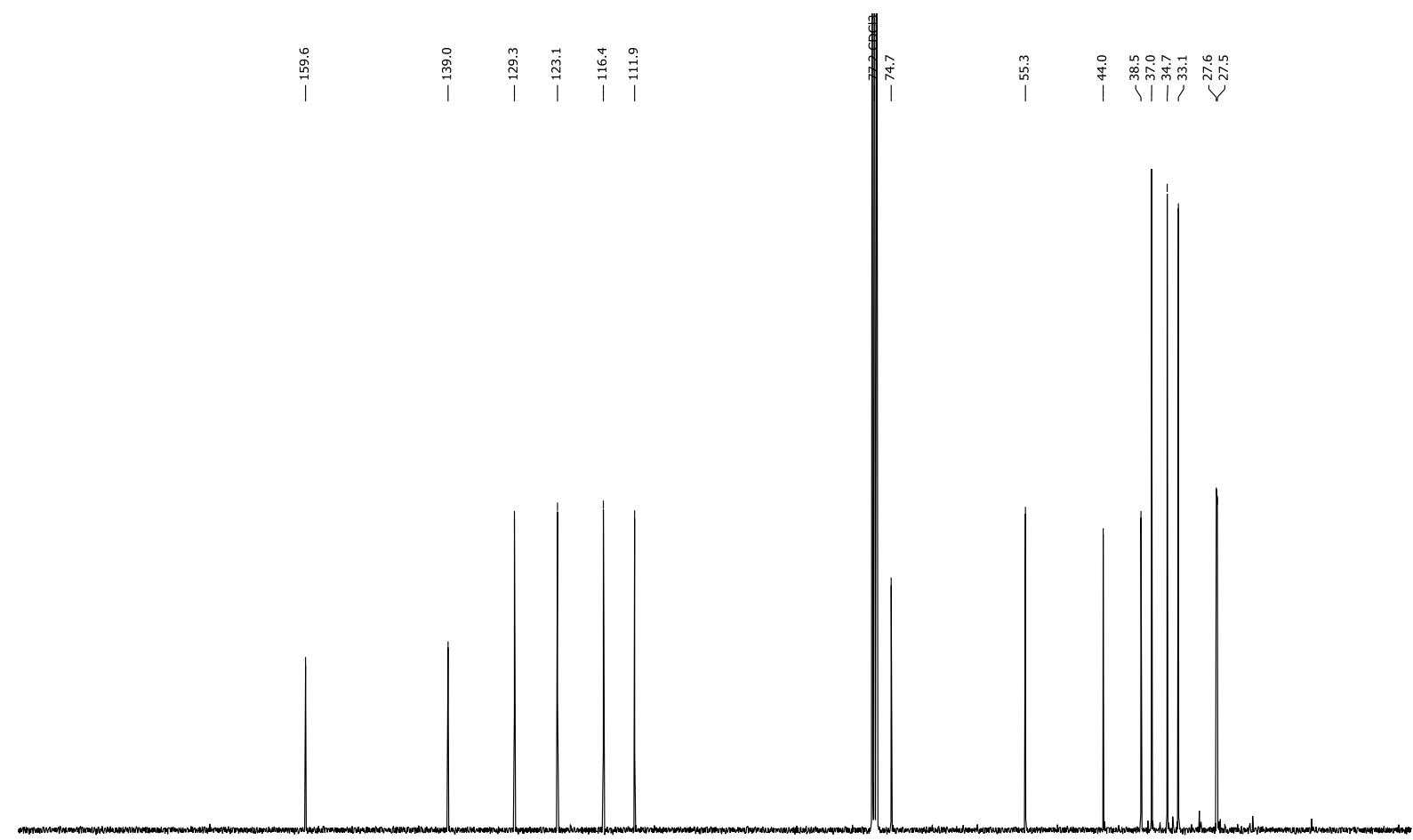


2-(4-(Methylthio)benzyl)adamantan-2-ol (7h)

${ }^{1} \mathrm{H}-\mathrm{NMR}$ (400 MHz, $\mathrm{CDCl}_{3}$ ):

范

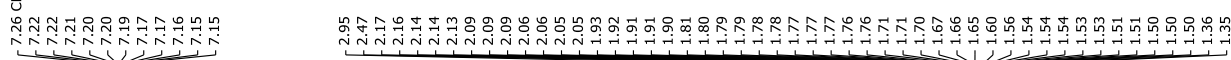

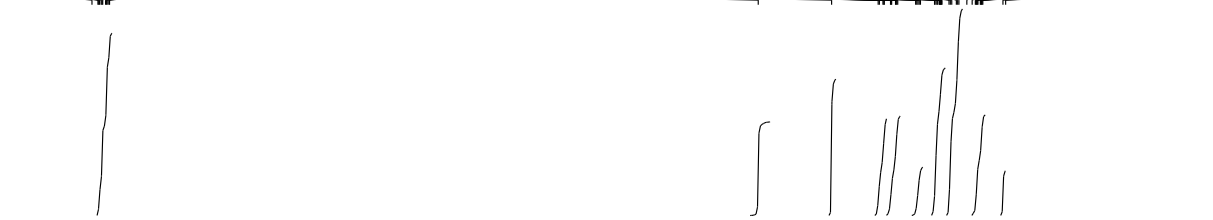<smiles>CS(=O)(=O)c1ccc(CC2(O)C3CC4CC(C3)CC2C4)cc1</smiles>

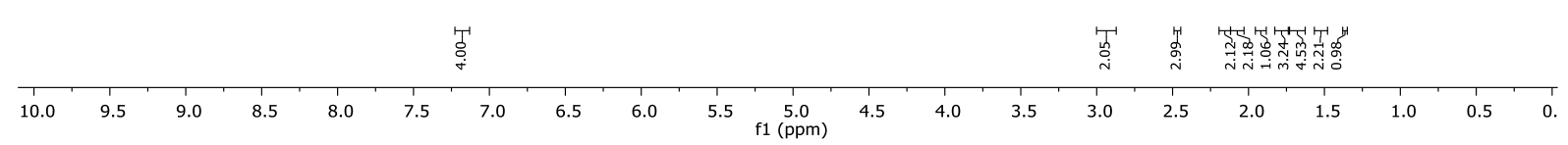

${ }^{13} \mathrm{C}-\mathrm{NMR}\left(100 \mathrm{MHz}, \mathrm{CDCl}_{3}\right)$ :

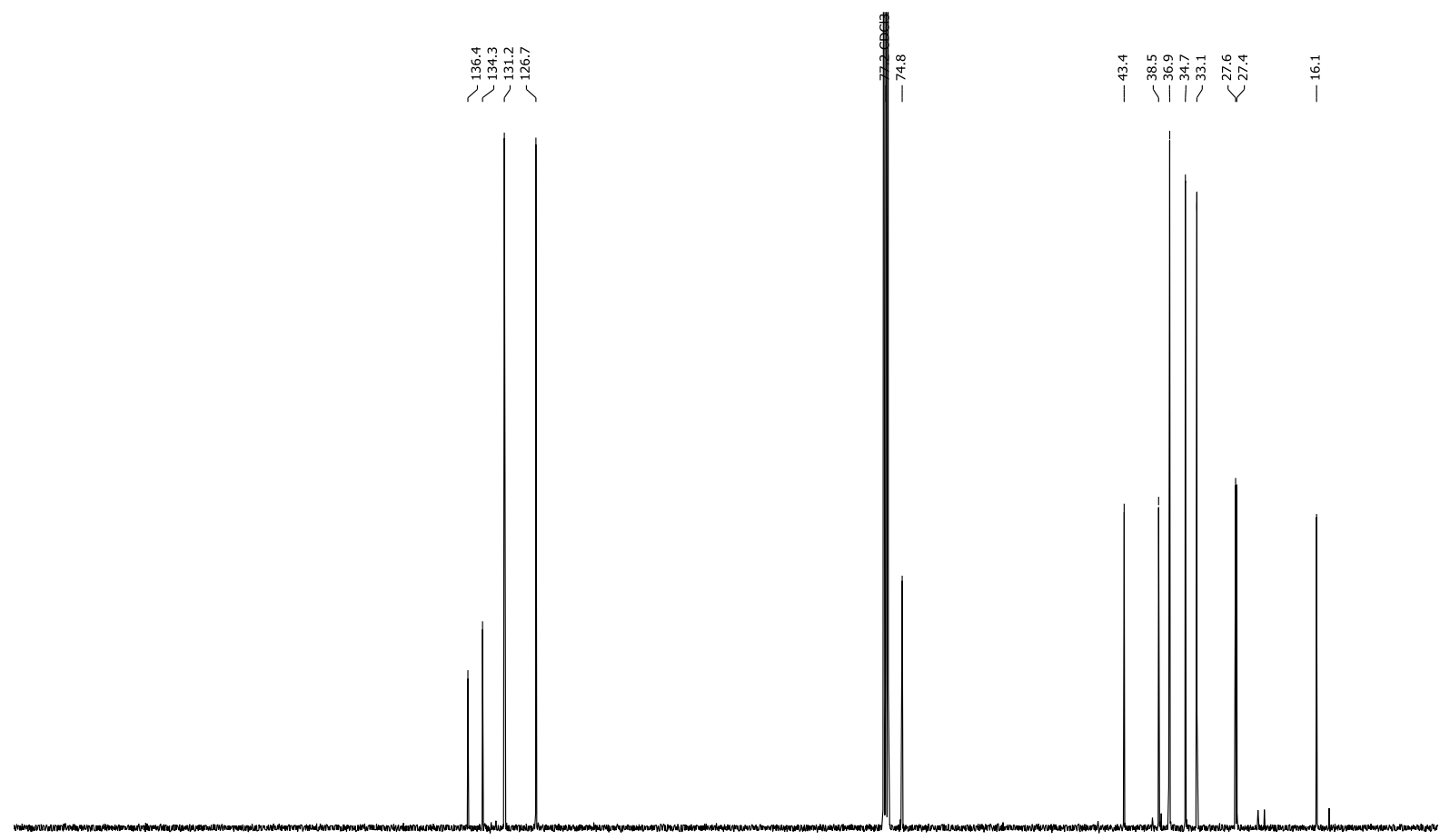

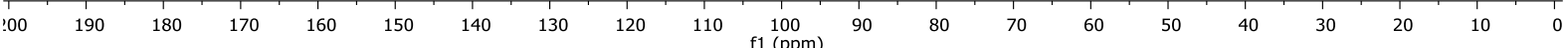


2-(2-Fluorophenyl)-1-phenylethan-1-ol (10a)

${ }^{1} \mathrm{H}-\mathrm{NMR}$ (400 MHz, $\mathrm{CDCl}_{3}$ ):

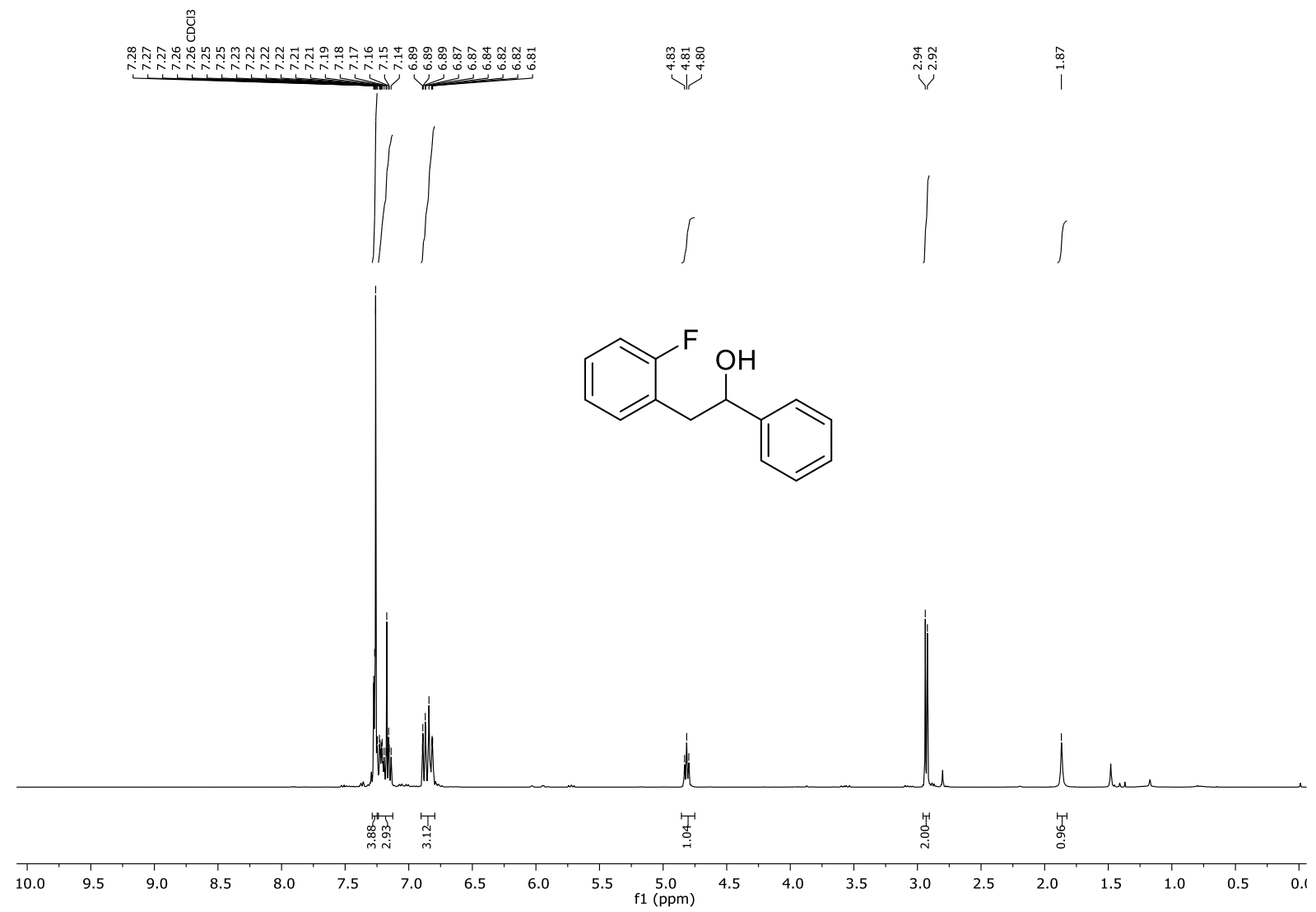

${ }^{13} \mathrm{C}-\mathrm{NMR}\left(100 \mathrm{MHz}, \mathrm{CDCl}_{3}\right)$ :

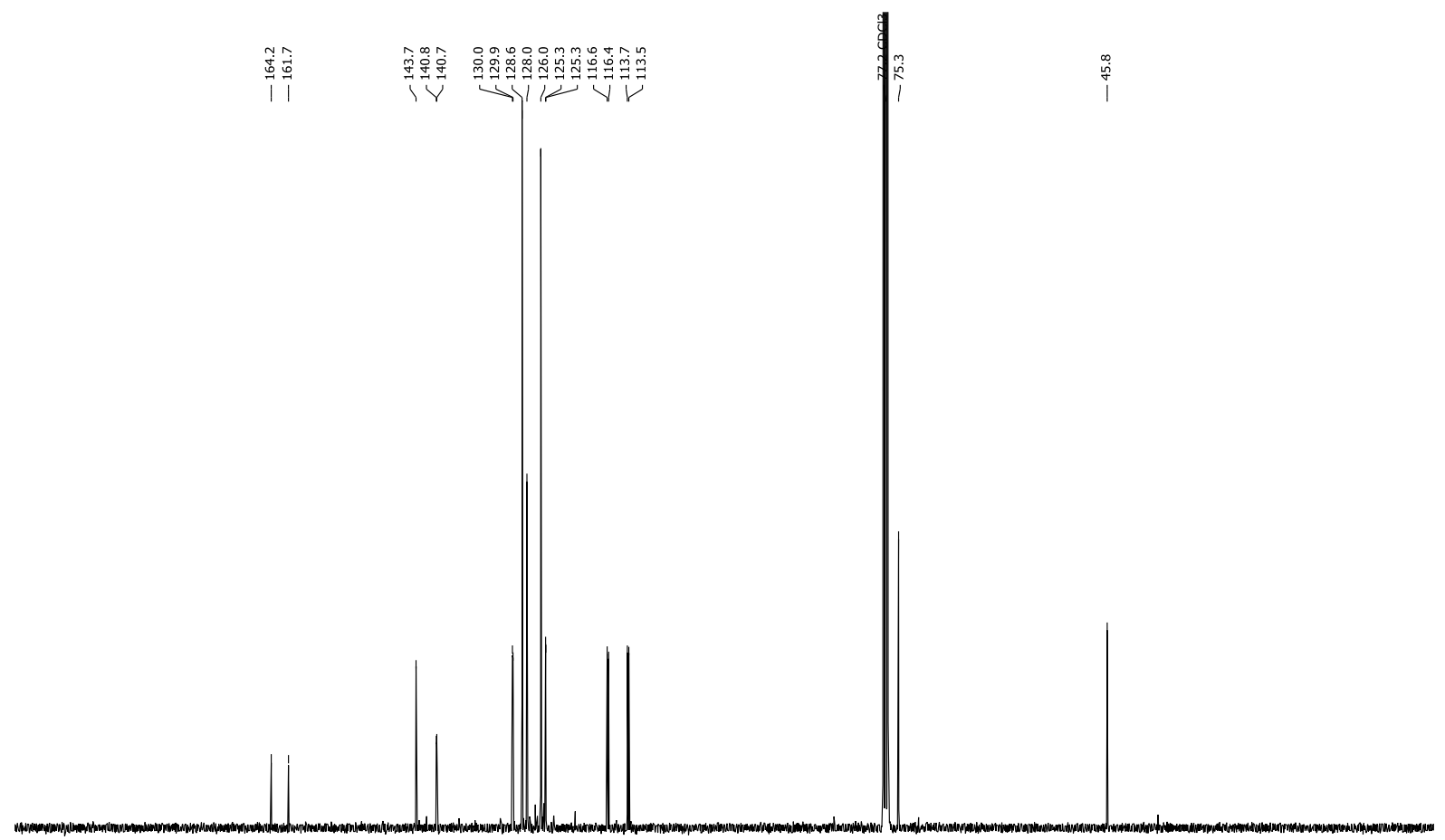

00

$\begin{array}{lllll}190 & 180 & 170 & 160 & 150\end{array}$

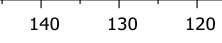

$110 \quad 100$

80

60

$\begin{array}{lllll}40 & 30 & 20 & 10 & \end{array}$ 
2-(2-Fluorophenyl)-1-(4-methoxyphenyl)ethan-1-ol (10b)

${ }^{1} \mathrm{H}-\mathrm{NMR}$ (400 MHz, $\left.\mathrm{CDCl}_{3}\right)$ :

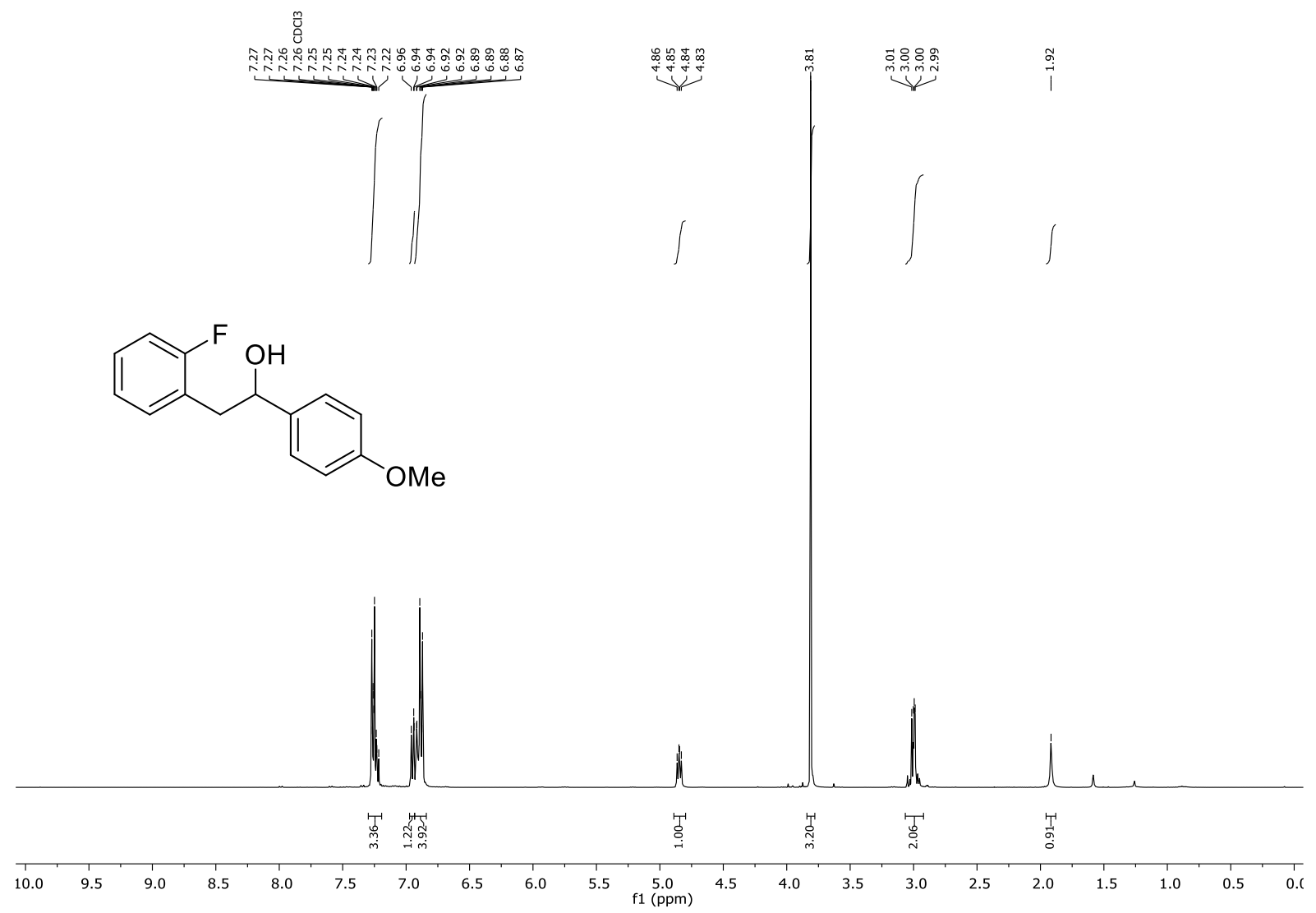

${ }^{13} \mathrm{C}-\mathrm{NMR}\left(100 \mathrm{MHz}, \mathrm{CDCl}_{3}\right)$ :

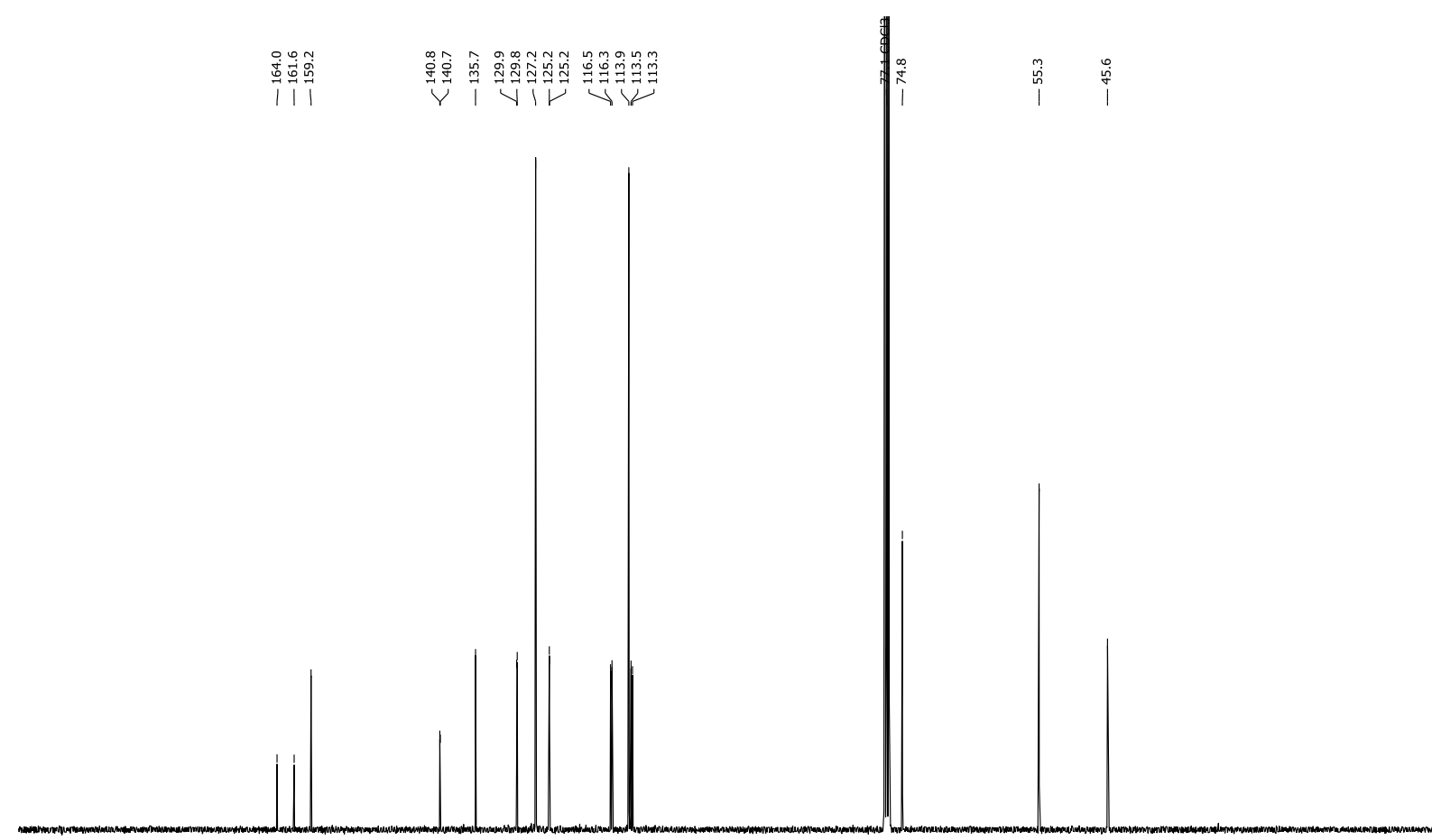


2-(2-Fluorobenzyl)adamantan-2-ol (10c)

${ }^{1} \mathrm{H}-\mathrm{NMR}$ (400 MHz, $\mathrm{CDCl}_{3}$ ):

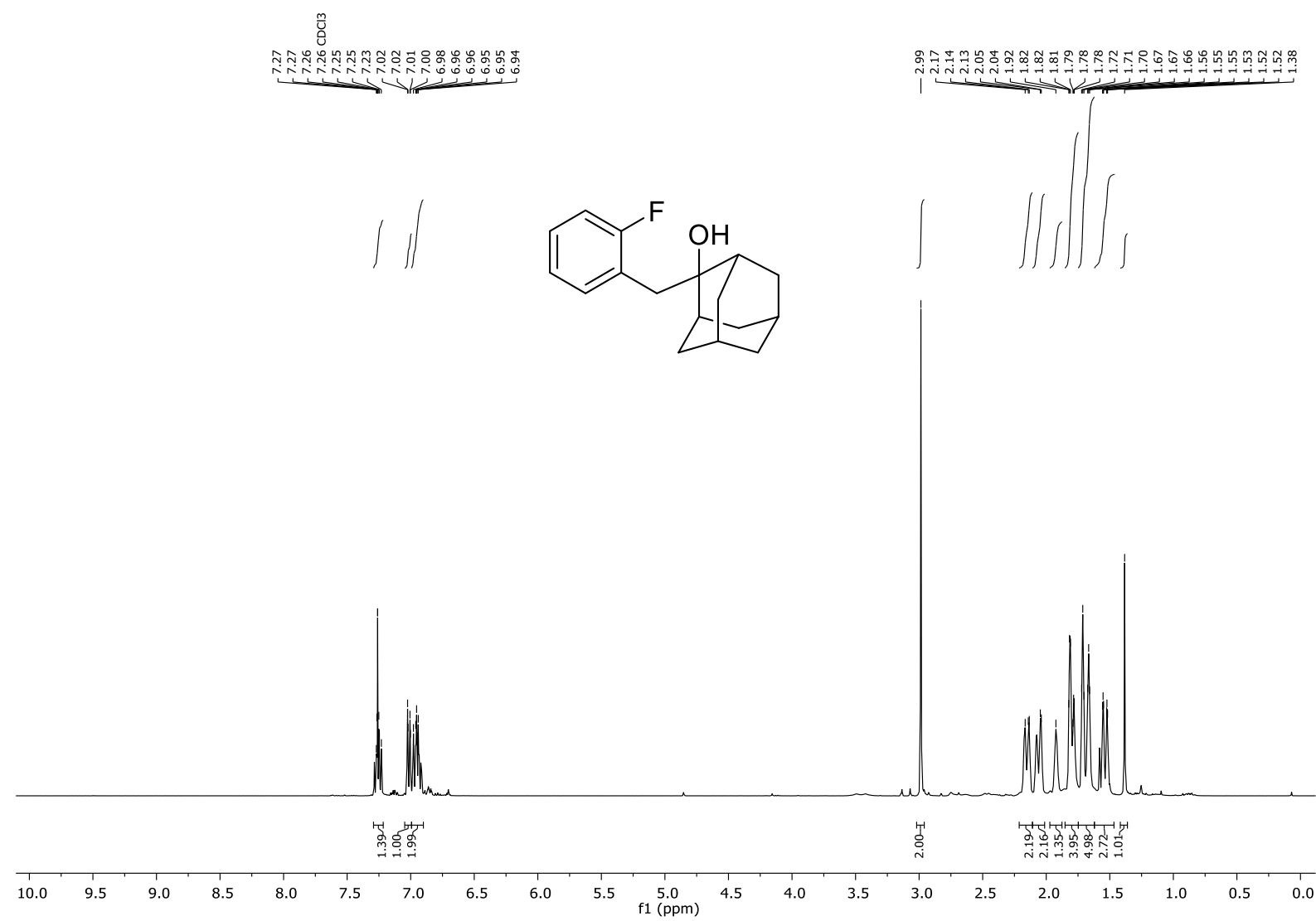

${ }^{13} \mathrm{C}-\mathrm{NMR}\left(100 \mathrm{MHz}, \mathrm{CDCl}_{3}\right)$ :

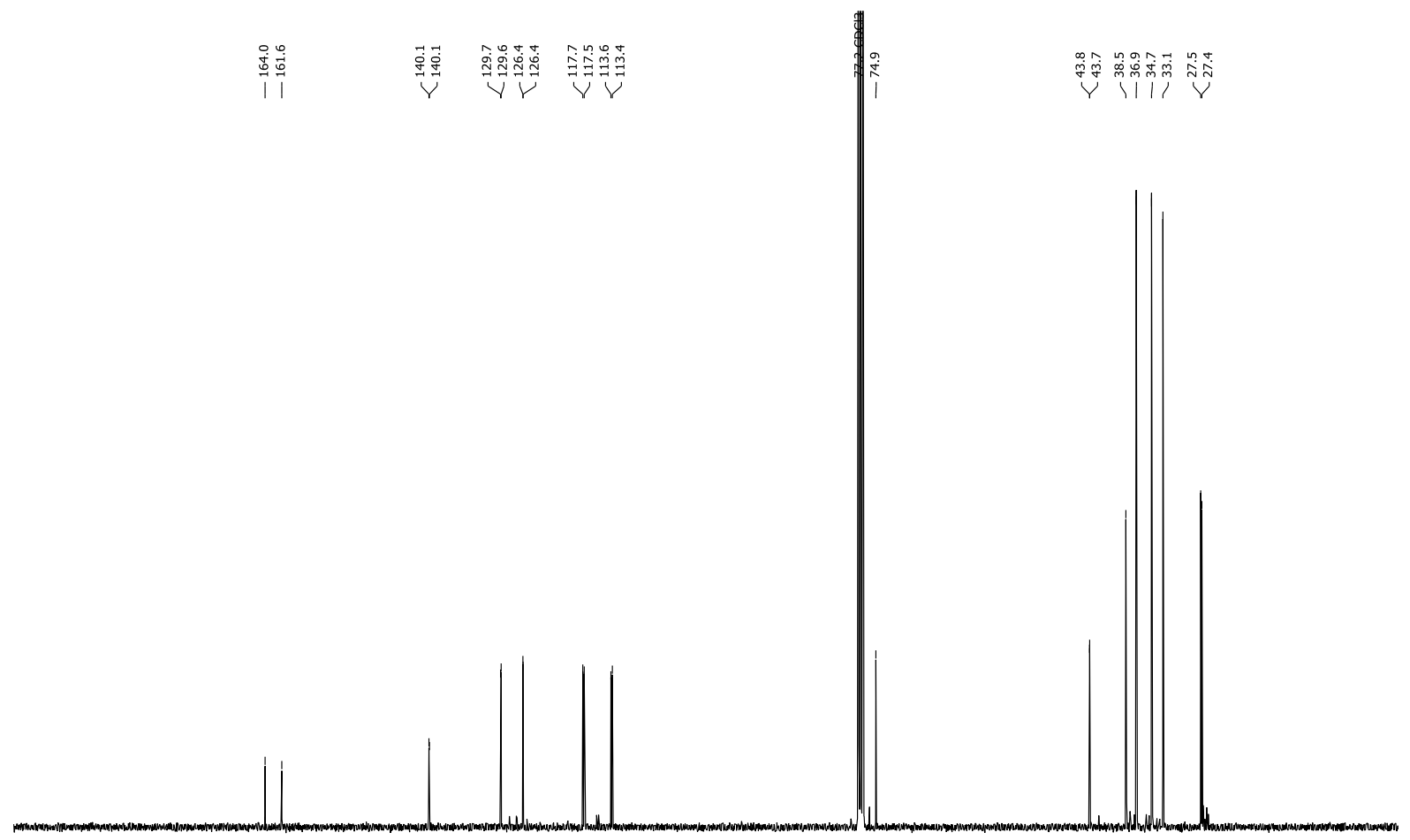

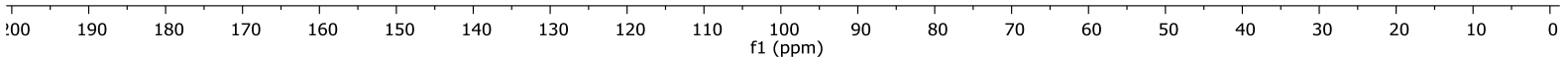


2-(2-Chlorophenyl)-1-phenylethan-1-ol (10d)

${ }^{1} \mathrm{H}-\mathrm{NMR}$ (400 MHz, $\mathrm{CDCl}_{3}$ ):

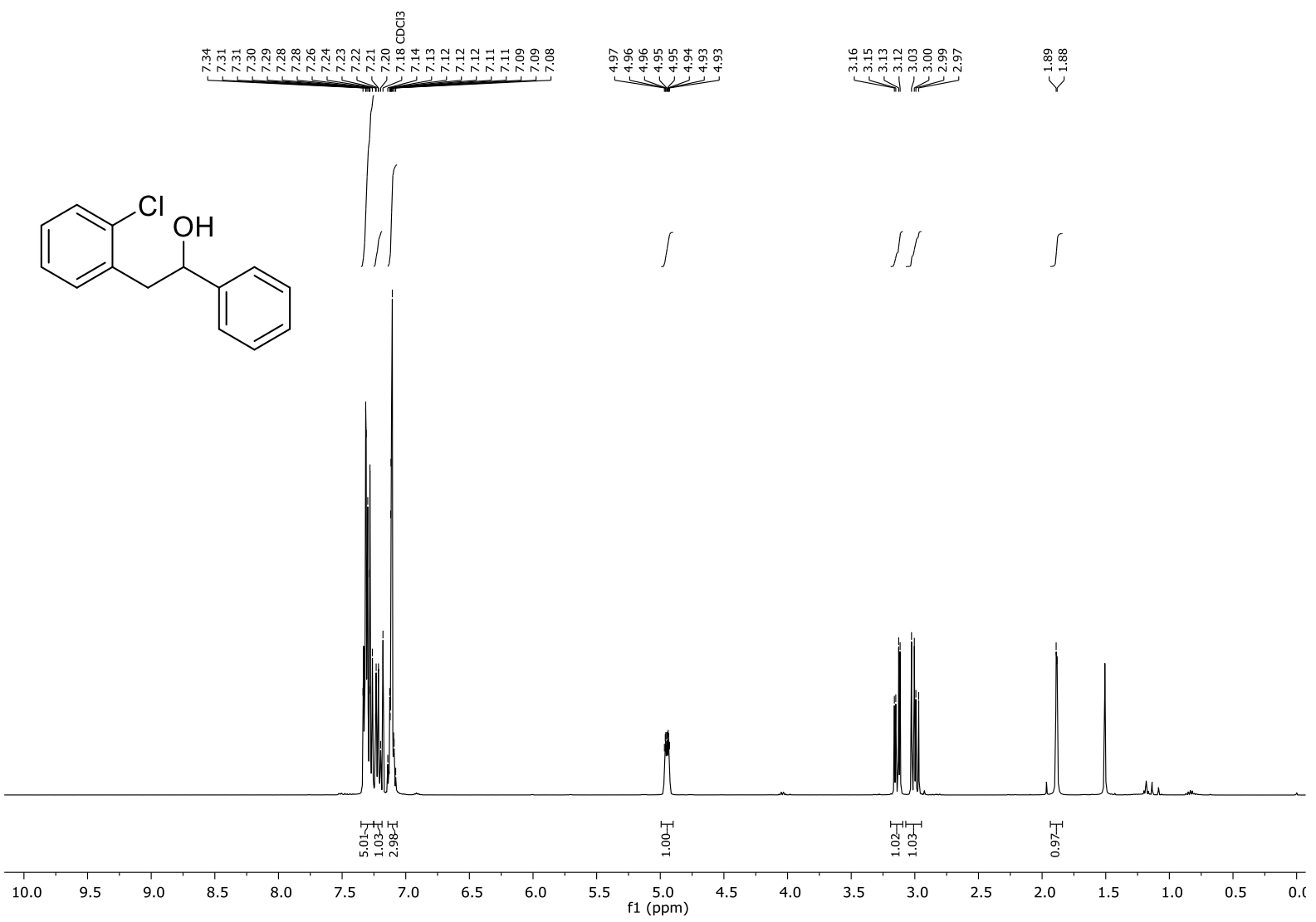

${ }^{13} \mathrm{C}-\mathrm{NMR}\left(100 \mathrm{MHz}, \mathrm{CDCl}_{3}\right)$ :

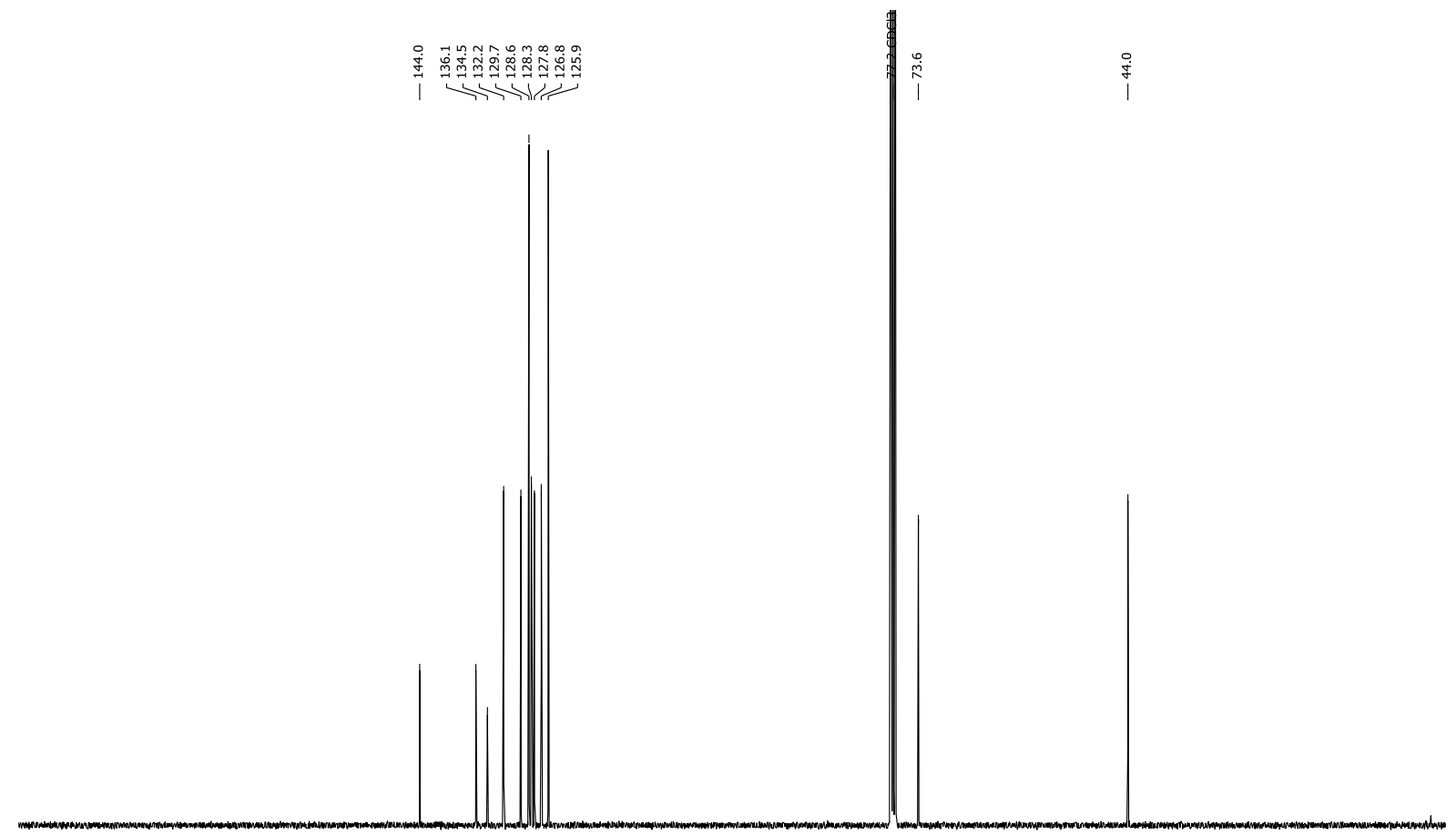

100

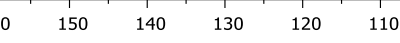

100

80 
1-Phenyl-2-(3-(trifluoromethyl)phenyl)ethan-1-ol (10e)

${ }^{1}$ H-NMR (400 MHz, $\mathrm{CDCl}_{3}$ ):

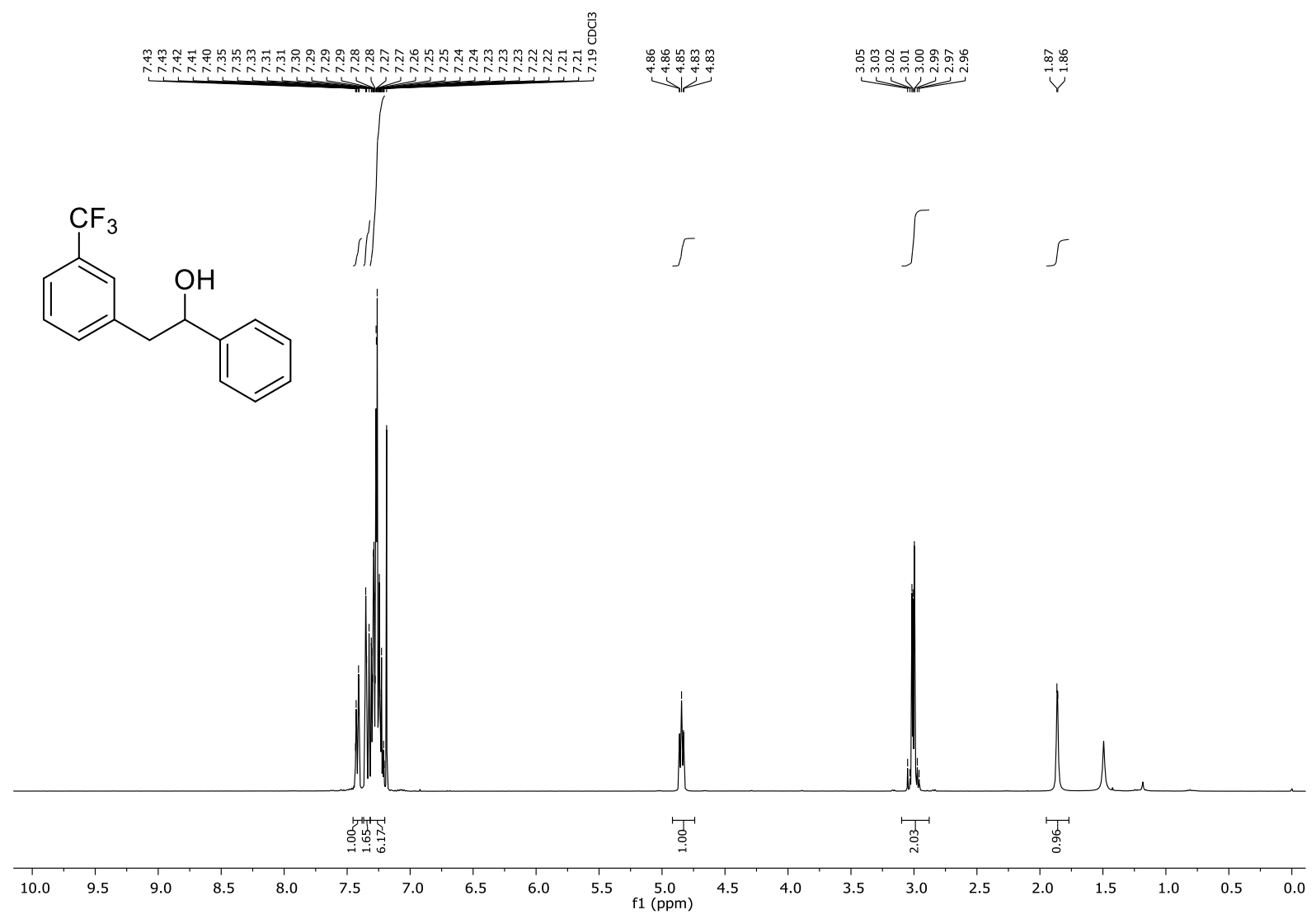

${ }^{13} \mathrm{C}-\mathrm{NMR}\left(100 \mathrm{MHz}, \mathrm{CDCl}_{3}\right)$ :

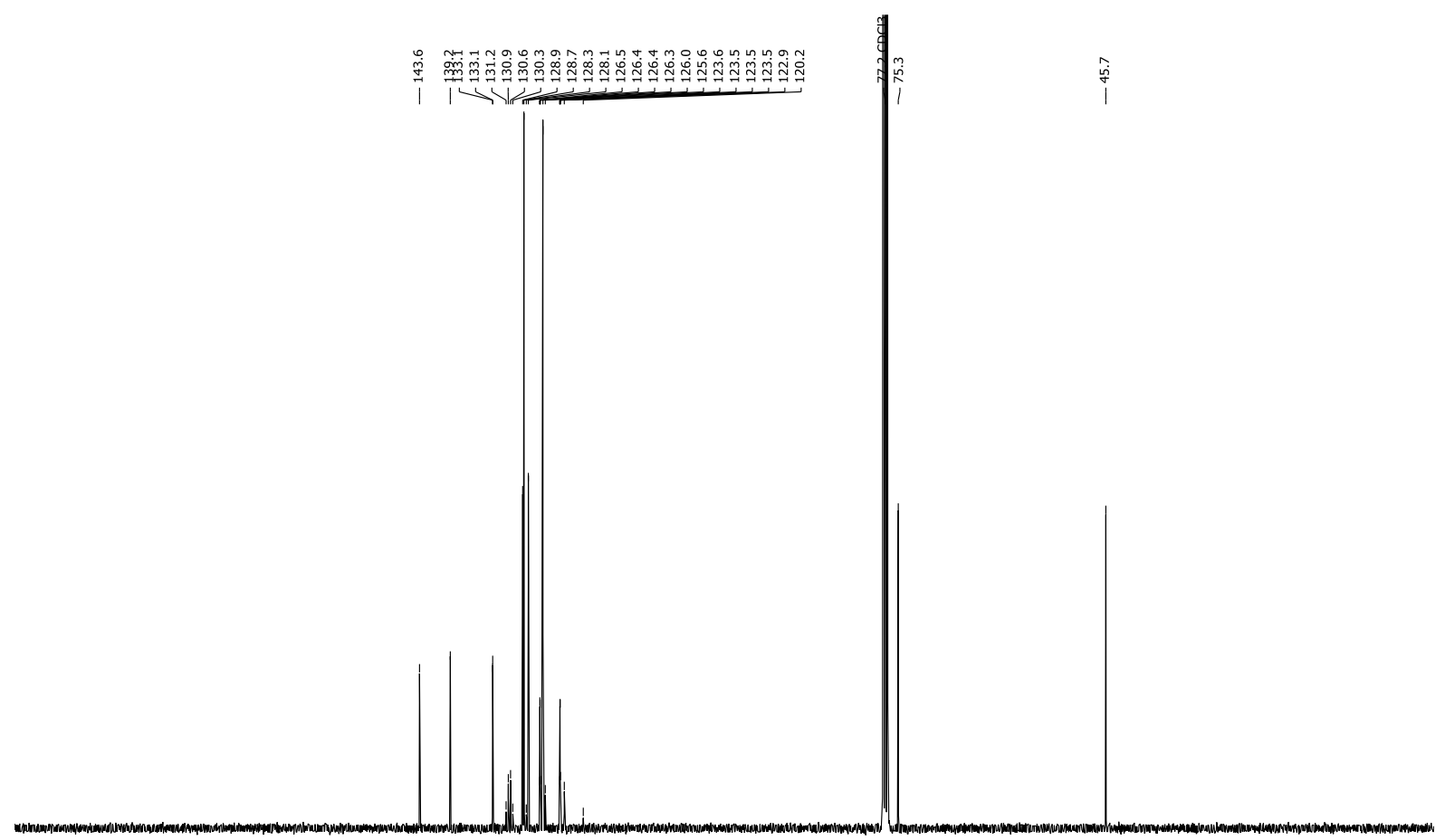


2-(6-Chloropyridin-3-yl)-1-phenylethan-1-ol (13a)

${ }^{1} \mathrm{H}-\mathrm{NMR}$ (400 MHz, $\left.\mathrm{CDCl}_{3}\right)$ :

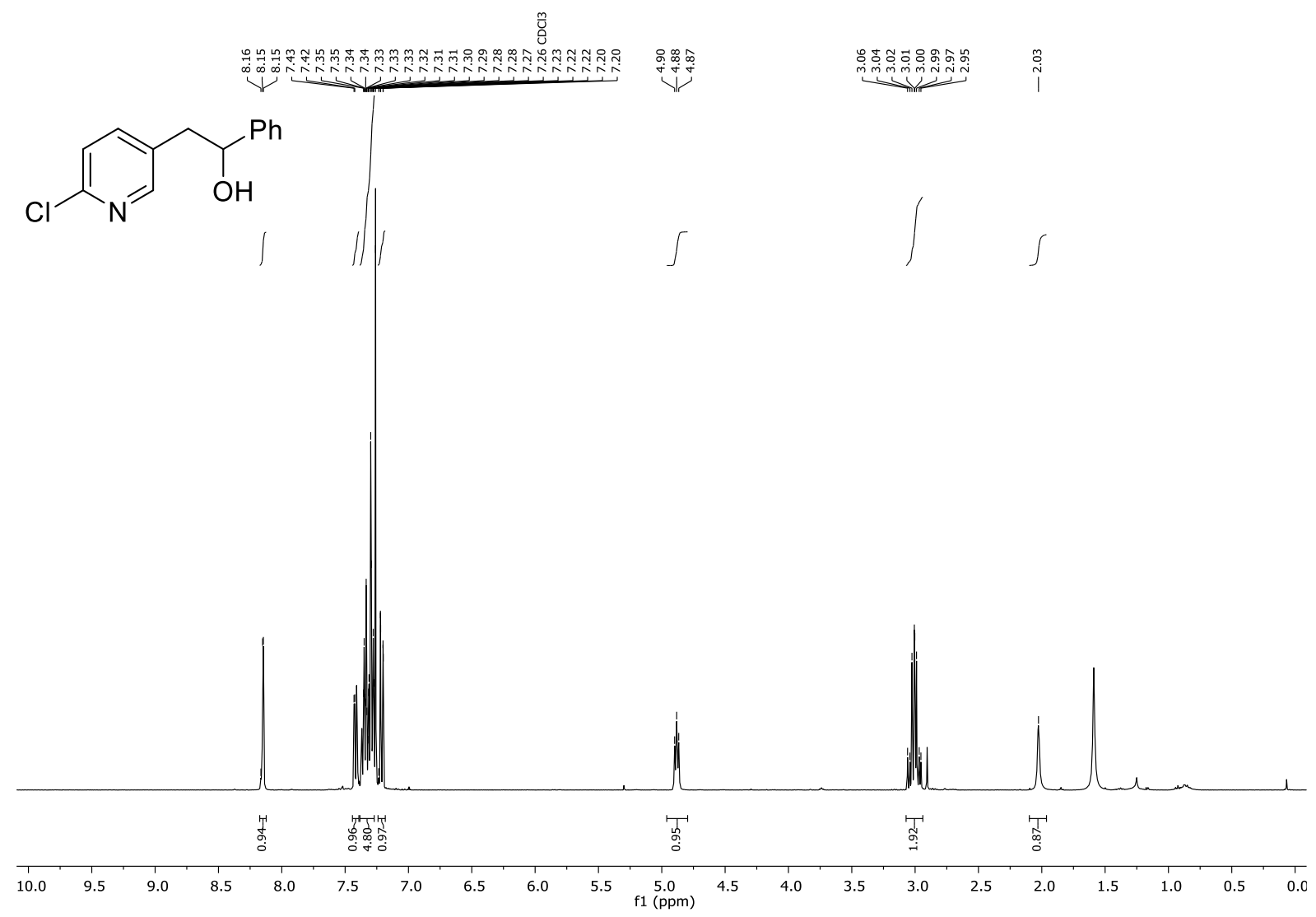

${ }^{13} \mathrm{C}-\mathrm{NMR}\left(100 \mathrm{MHz}, \mathrm{CDCl}_{3}\right)$ :

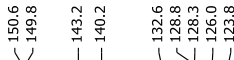

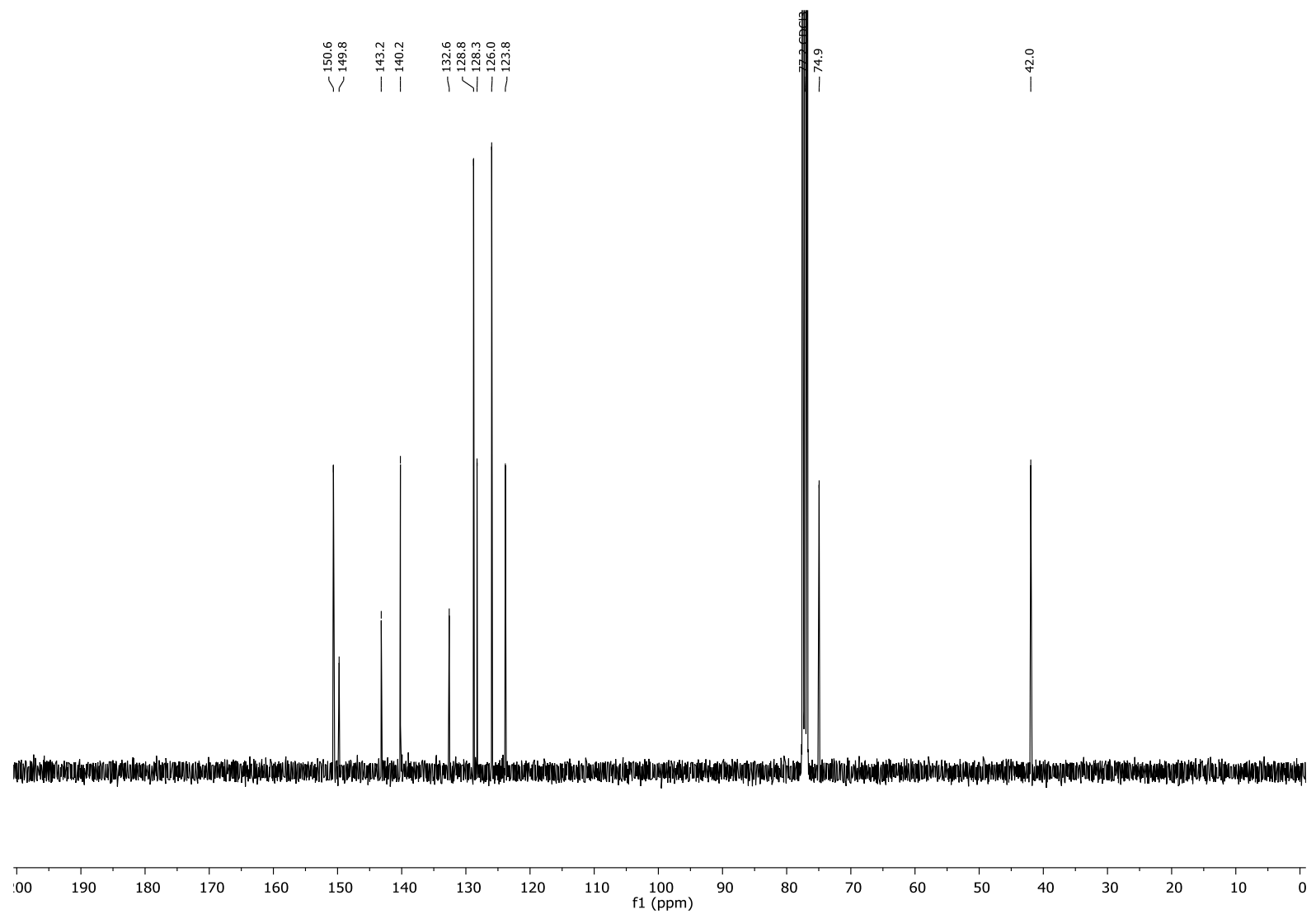


2-(6-Chloro-2-fluoropyridin-3-yl)-1-(2,6-dichlorophenyl)ethan-1-ol (13b)

${ }^{1} \mathrm{H}-\mathrm{NMR}\left(400 \mathrm{MHz}, \mathrm{CDCl}_{3}\right)$ :

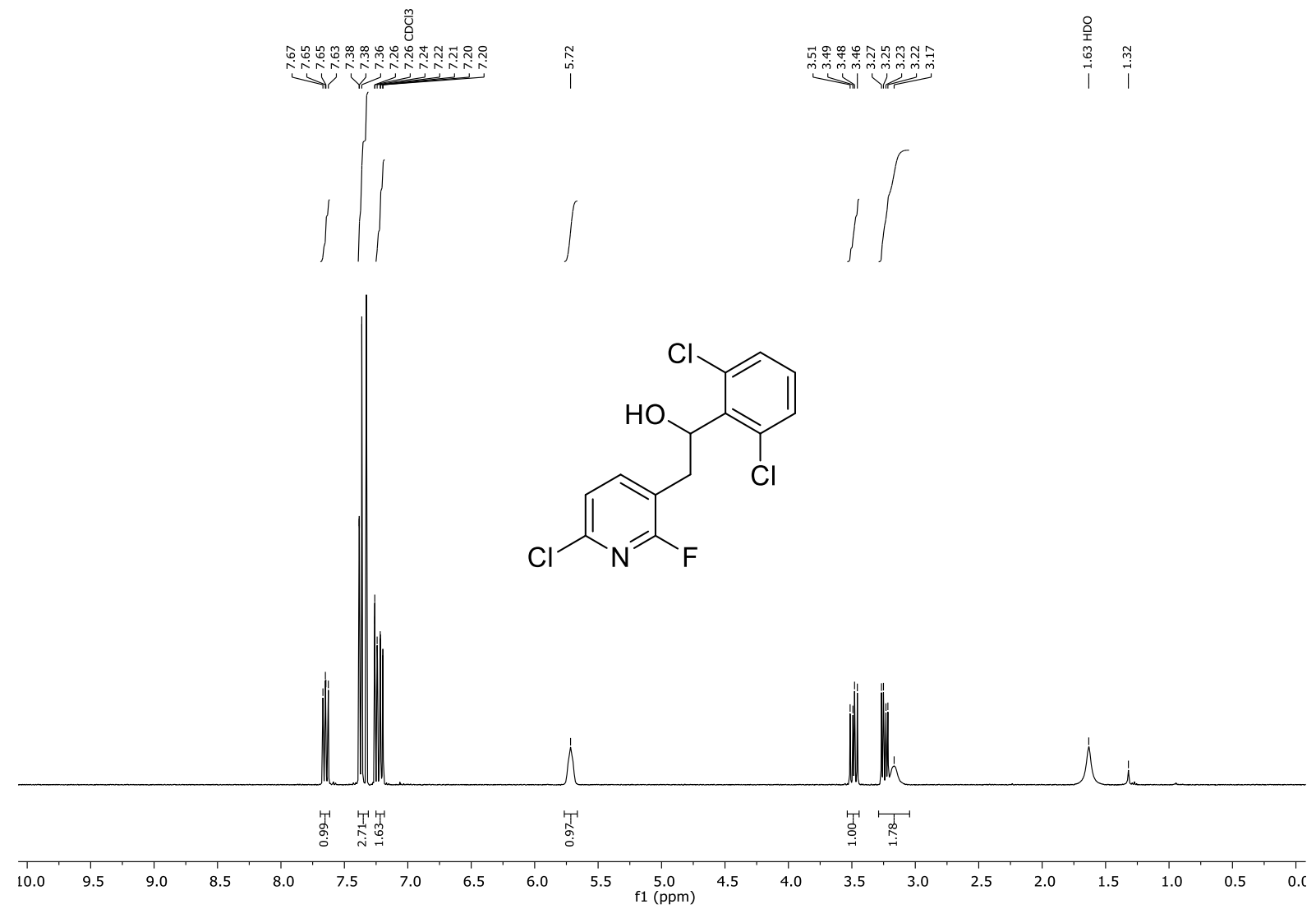

${ }^{13} \mathrm{C}-\mathrm{NMR}\left(100 \mathrm{MHz}, \mathrm{CDCl}_{3}\right)$ :

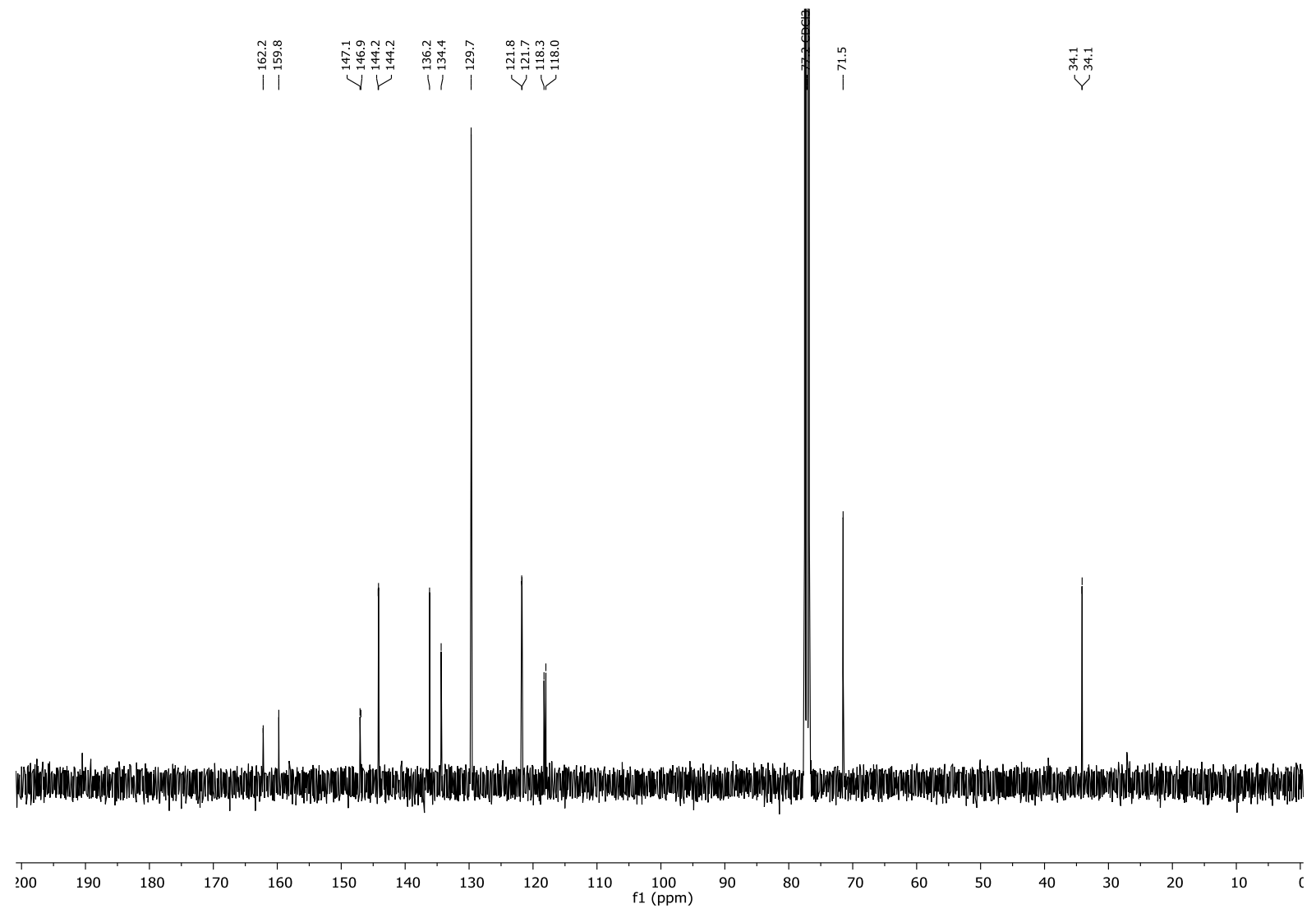


2-(6-Chloro-2-(methylthio)pyridin-3-yl)-1-(4-methoxyphenyl)ethan-1-ol (13c)

${ }^{1} \mathrm{H}-\mathrm{NMR}\left(400 \mathrm{MHz}, \mathrm{CDCl}_{3}\right)$ :

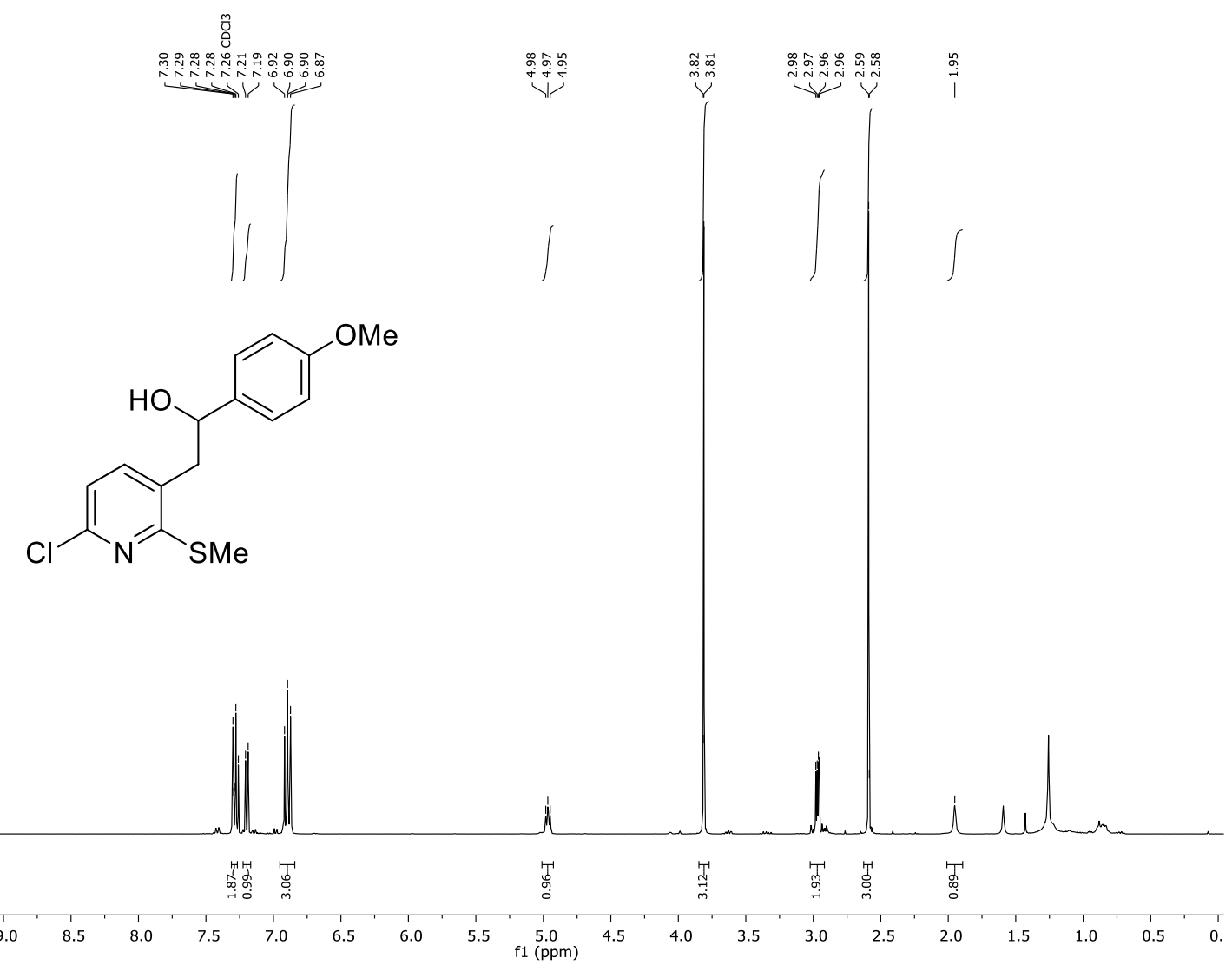

${ }^{13} \mathrm{C}-\mathrm{NMR}\left(100 \mathrm{MHz}, \mathrm{CDCl}_{3}\right)$ :
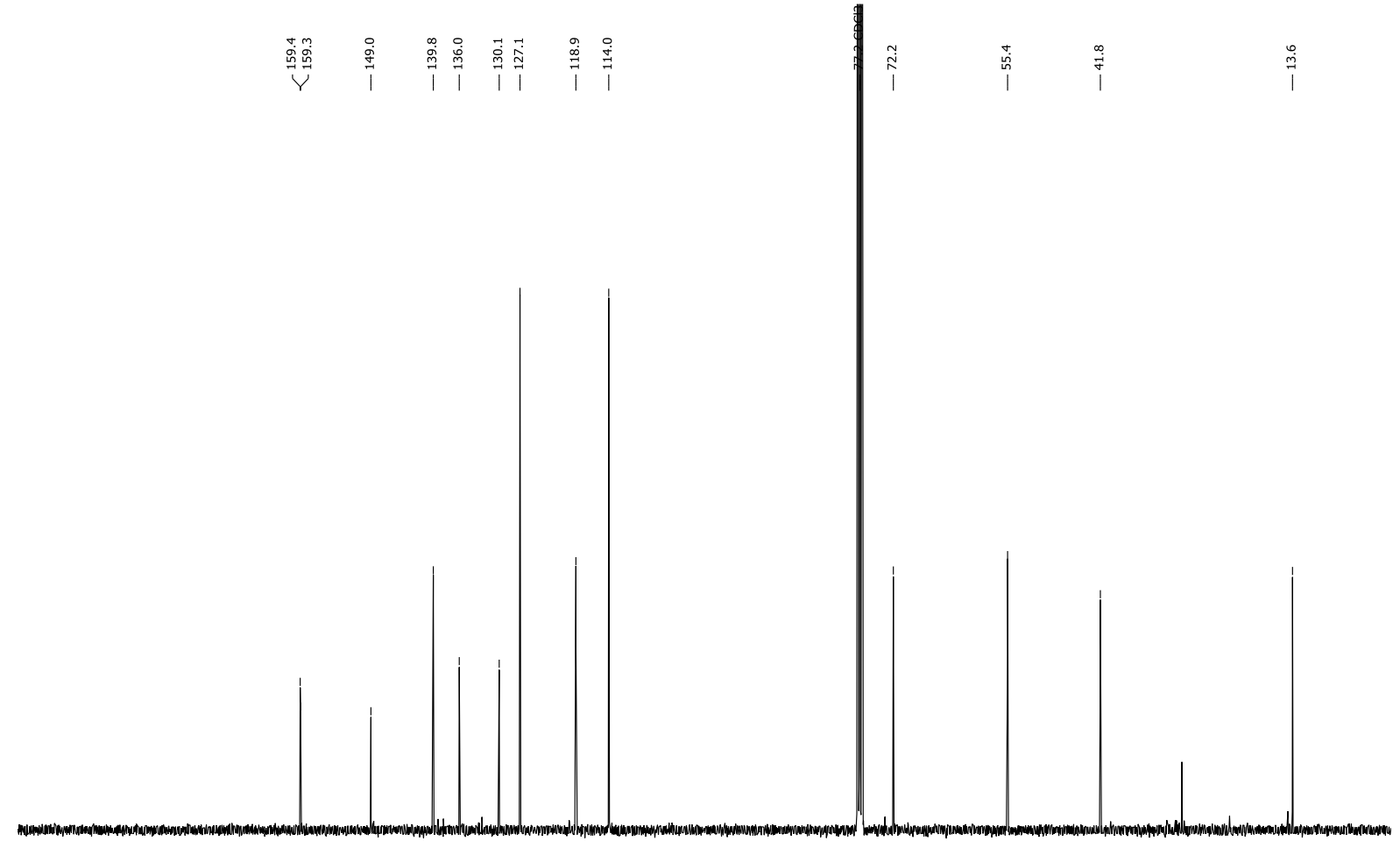
2-(6-Chloro-2-(methylthio)pyridin-3-yl)-1-(2,6-dichlorophenyl)ethan-1-ol (13d)

${ }^{1} \mathrm{H}-\mathrm{NMR}\left(400 \mathrm{MHz}, \mathrm{CDCl}_{3}\right)$ :

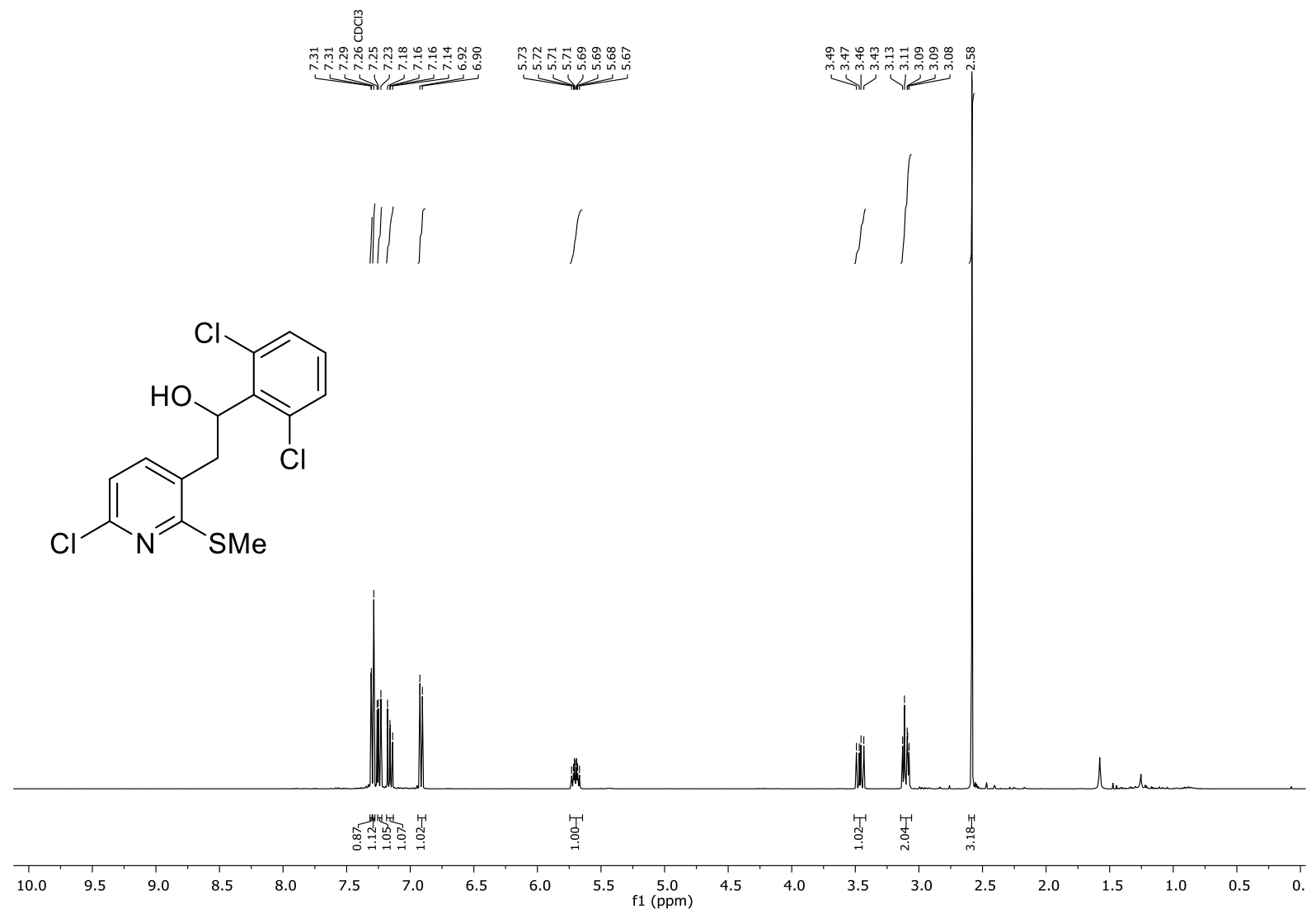

${ }^{13} \mathrm{C}-\mathrm{NMR}\left(100 \mathrm{MHz}, \mathrm{CDCl}_{3}\right)$ :

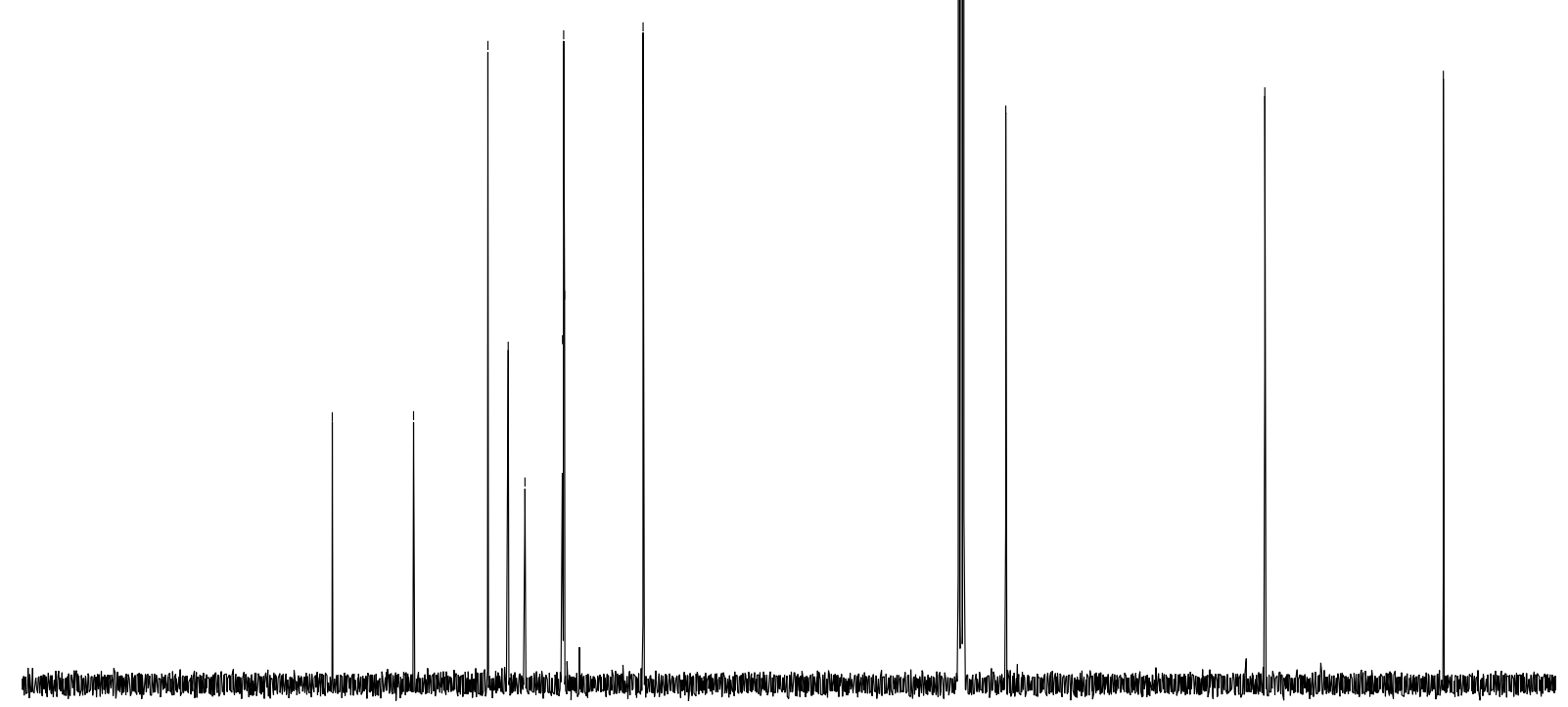

100

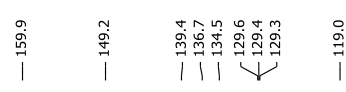

$\| \stackrel{m}{1}$ 
2-(6-Chloro-2-(methylthio)pyridin-3-yl)-1-(4-chlorophenyl)ethan-1-ol (13e)

${ }^{1} \mathrm{H}-\mathrm{NMR}\left(400 \mathrm{MHz}, \mathrm{CDCl}_{3}\right)$ :
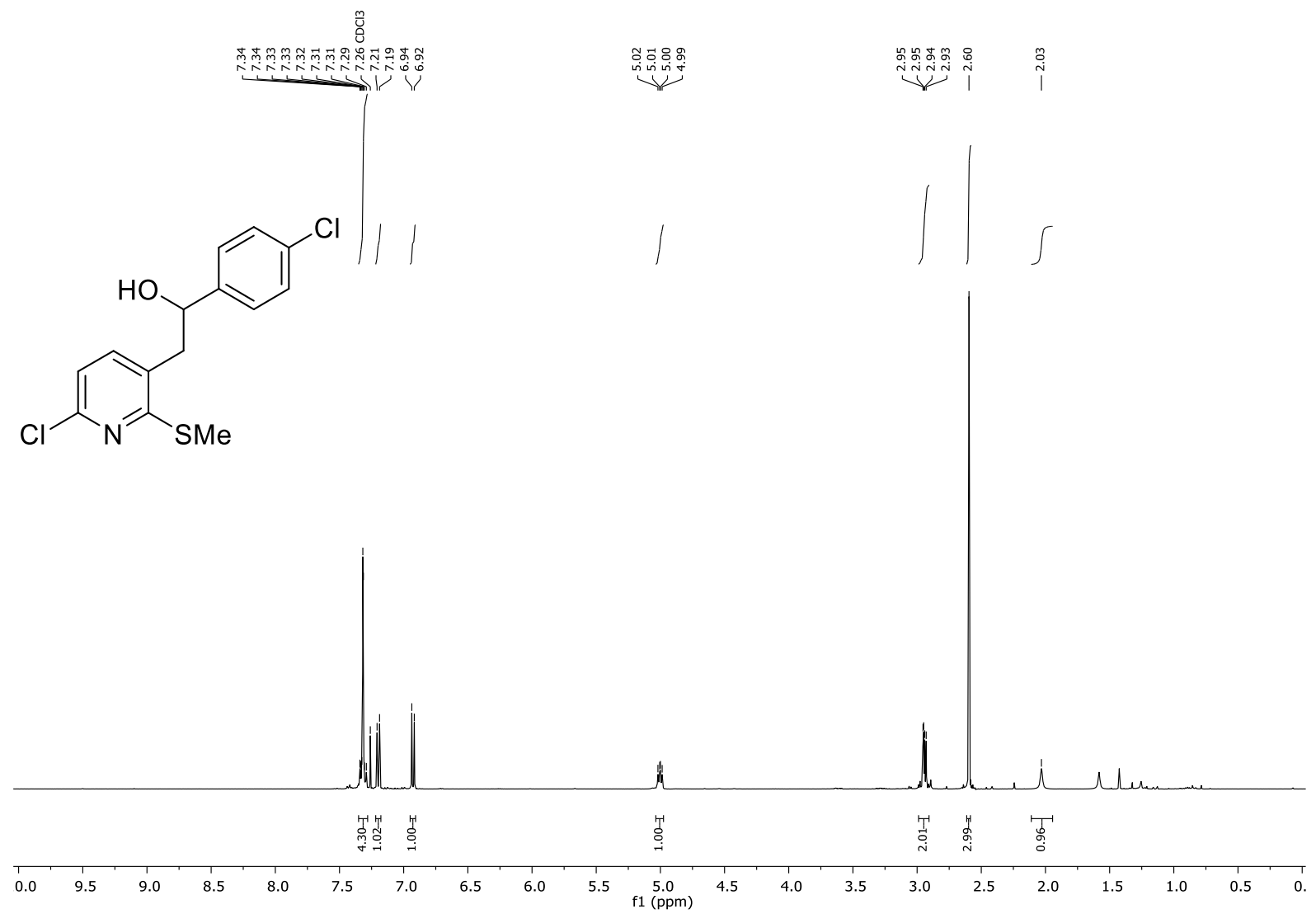

${ }^{13} \mathrm{C}-\mathrm{NMR}\left(100 \mathrm{MHz}, \mathrm{CDCl}_{3}\right)$ :

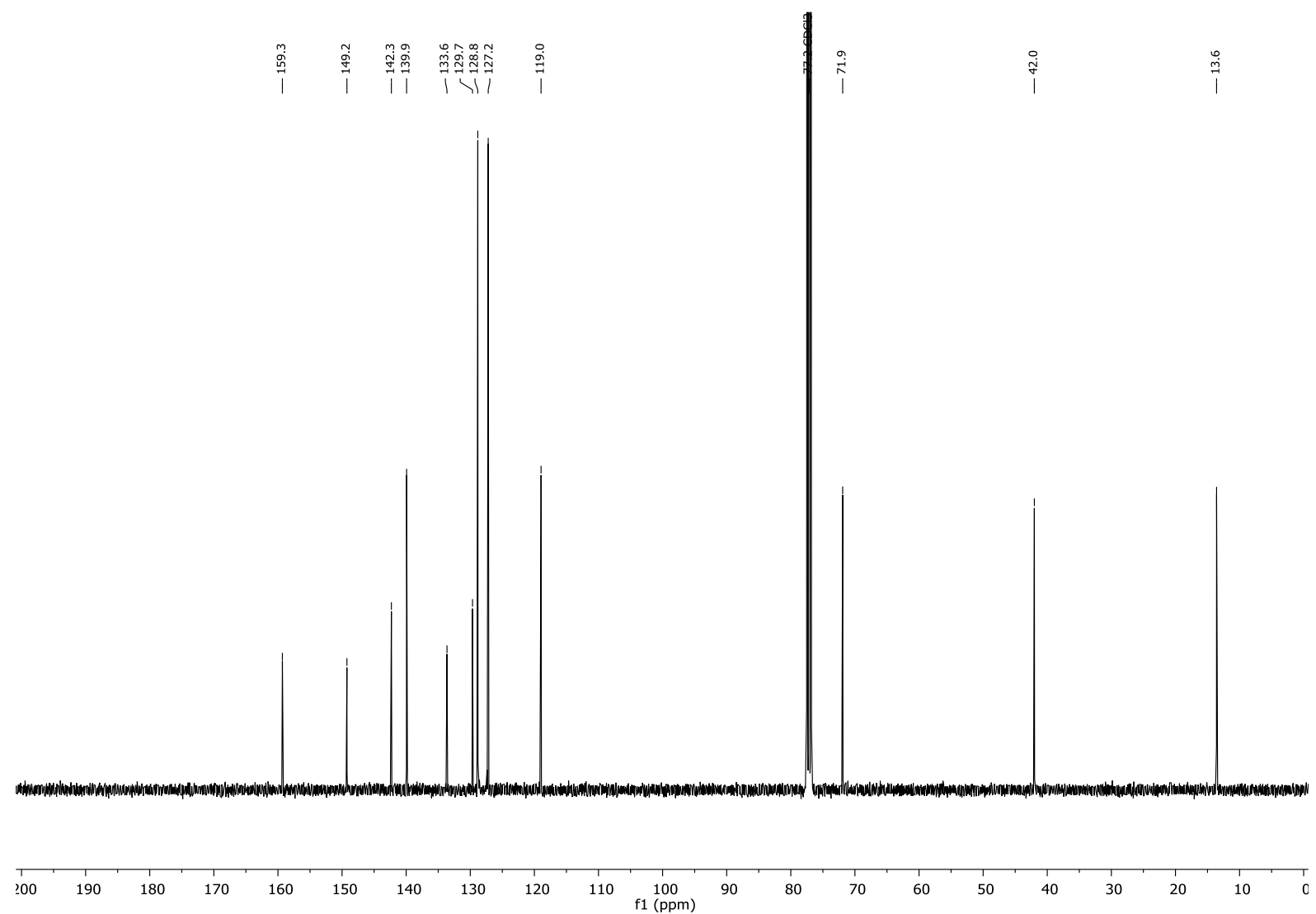


Starting materials:

(Iodomethyl)benzene (1a)

${ }^{1} \mathrm{H}-\mathrm{NMR}$ (400 MHz, $\mathrm{CDCl}_{3}$ ):

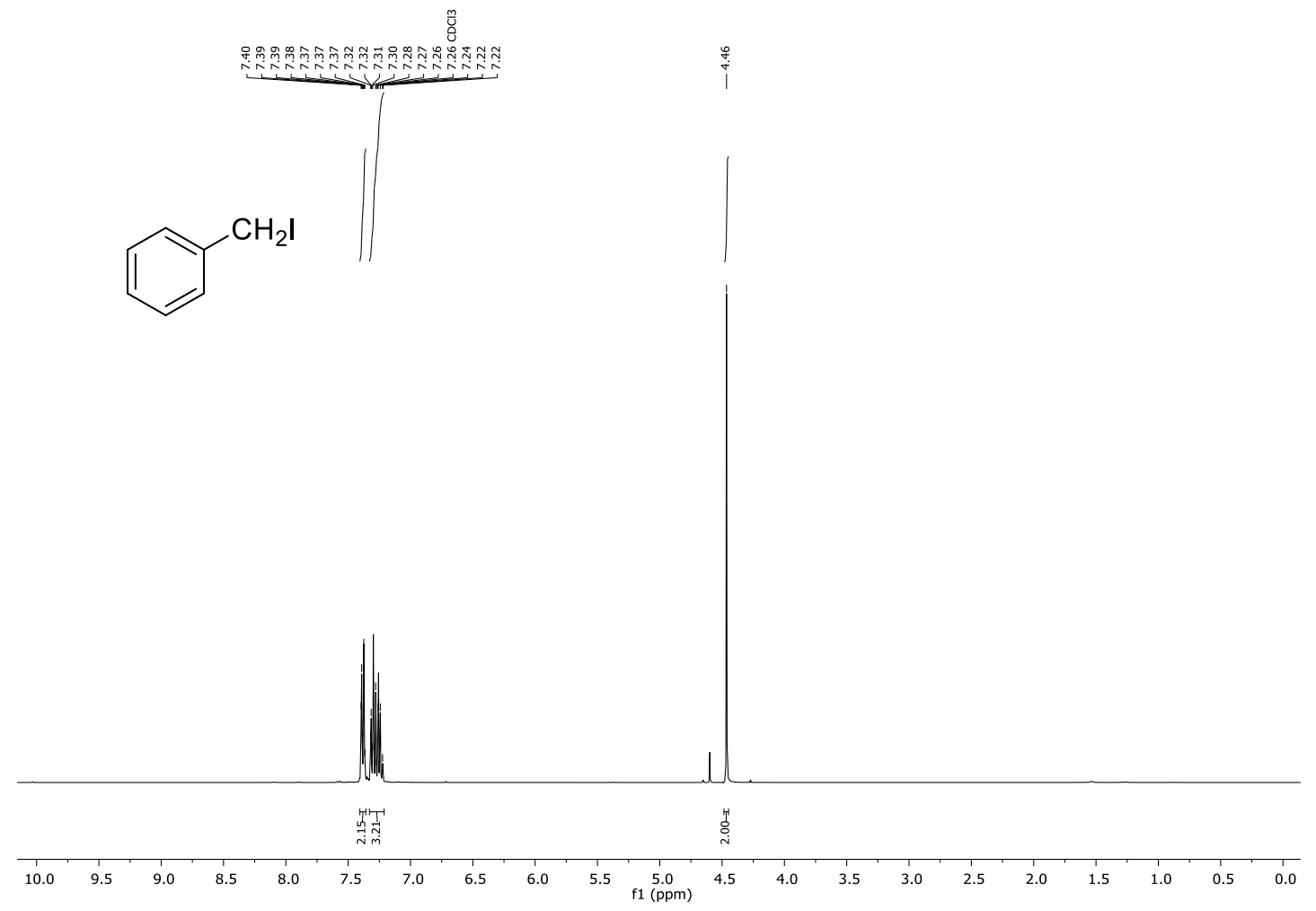

${ }^{13} \mathrm{C}-\mathrm{NMR}$ (100 MHz, $\left.\mathrm{CDCl}_{3}\right)$ :

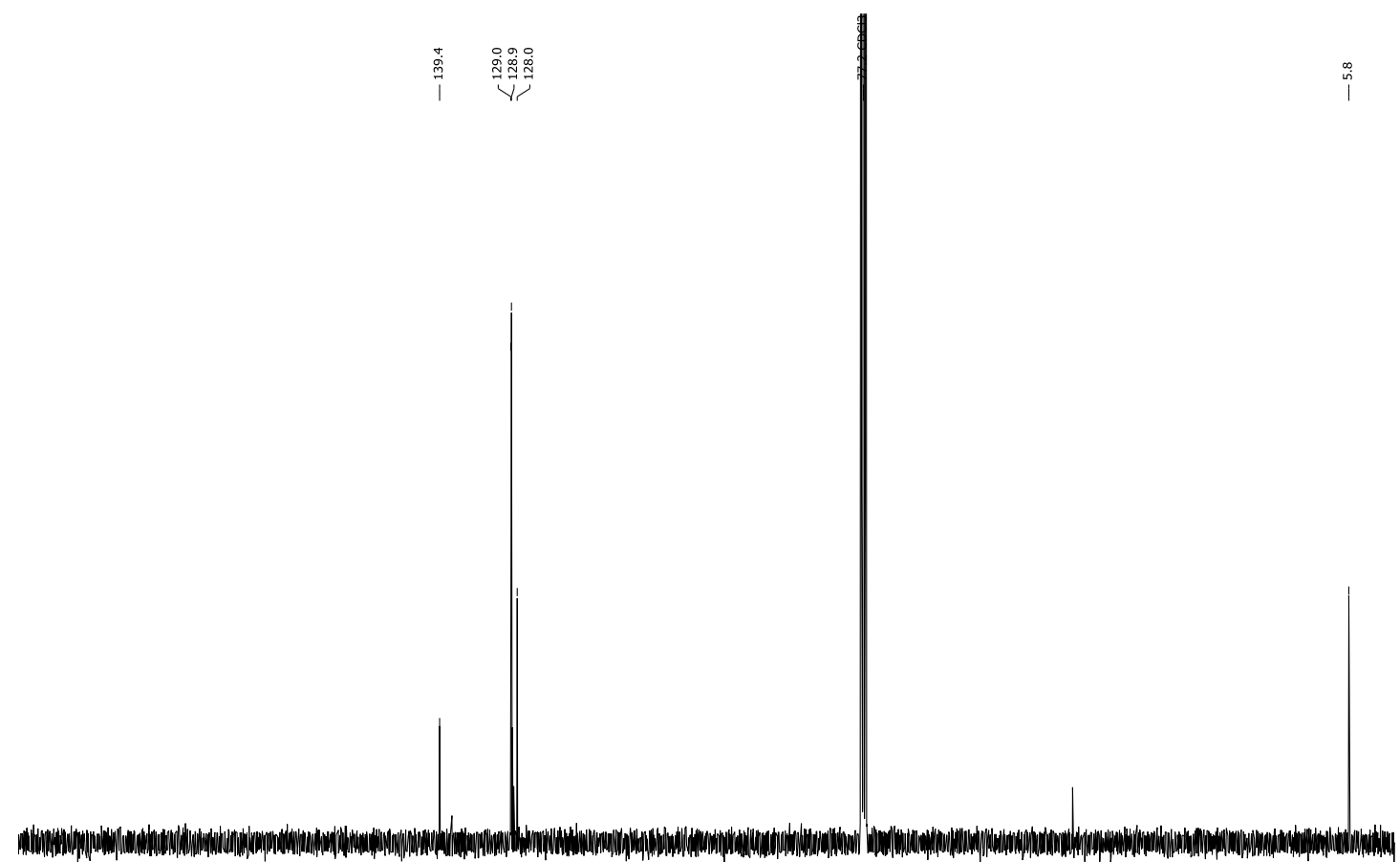

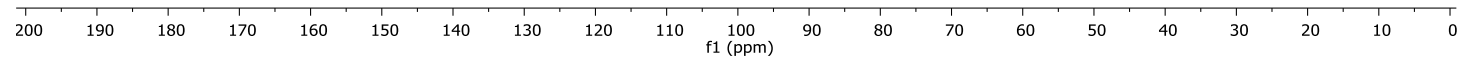


1-(Tert-butyl)-4-(iodomethyl)benzene (1b)

${ }^{1} \mathrm{H}-\mathrm{NMR}$ (400 MHz, $\left.\mathrm{CDCl}_{3}\right)$ :

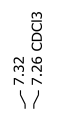

$$
\stackrel{\substack{+⿱ 亠 䒑 十}}{\mid}
$$<smiles>CC(C)(C)c1ccc(CI)cc1</smiles>
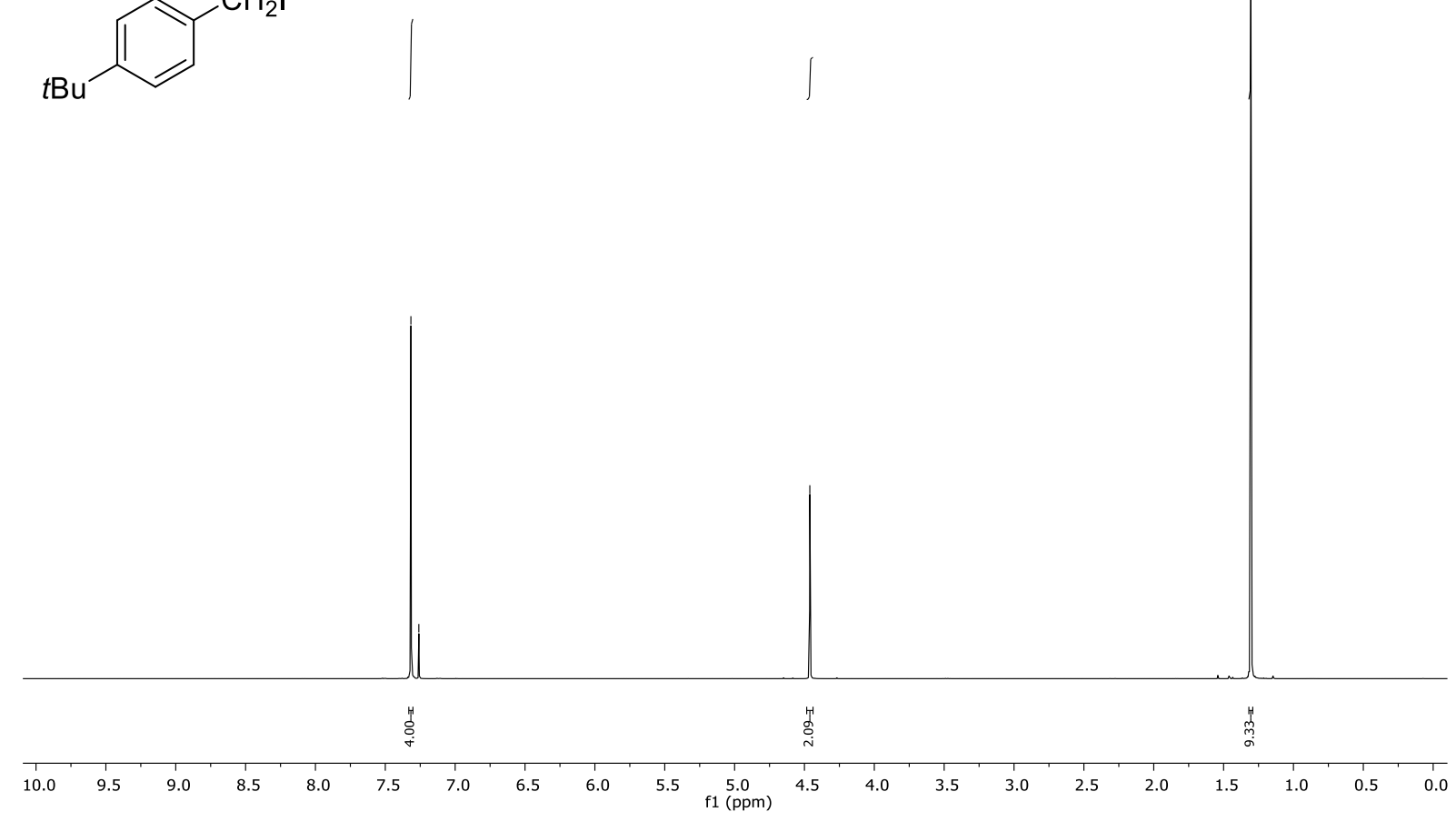

${ }^{13} \mathrm{C}-\mathrm{NMR}\left(100 \mathrm{MHz}, \mathrm{CDCl}_{3}\right)$ :

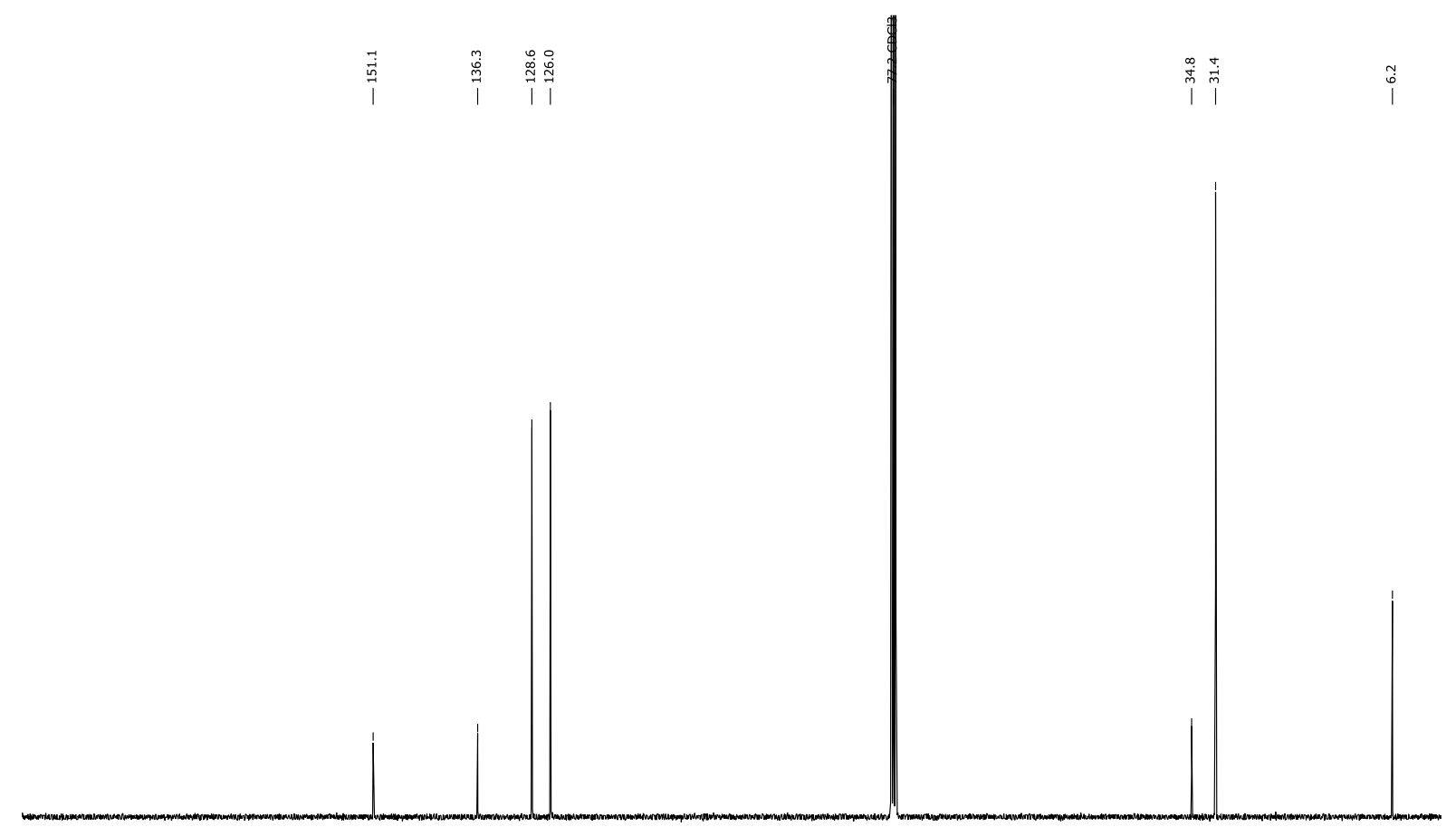

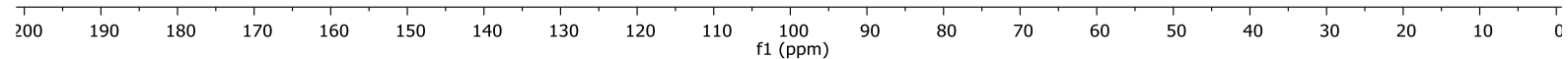


1-(Iodomethyl)-4-isopropylbenzene (1c)

${ }^{1} \mathrm{H}-\mathrm{NMR}$ (400 MHz, $\mathrm{CDCl}_{3}$ ):

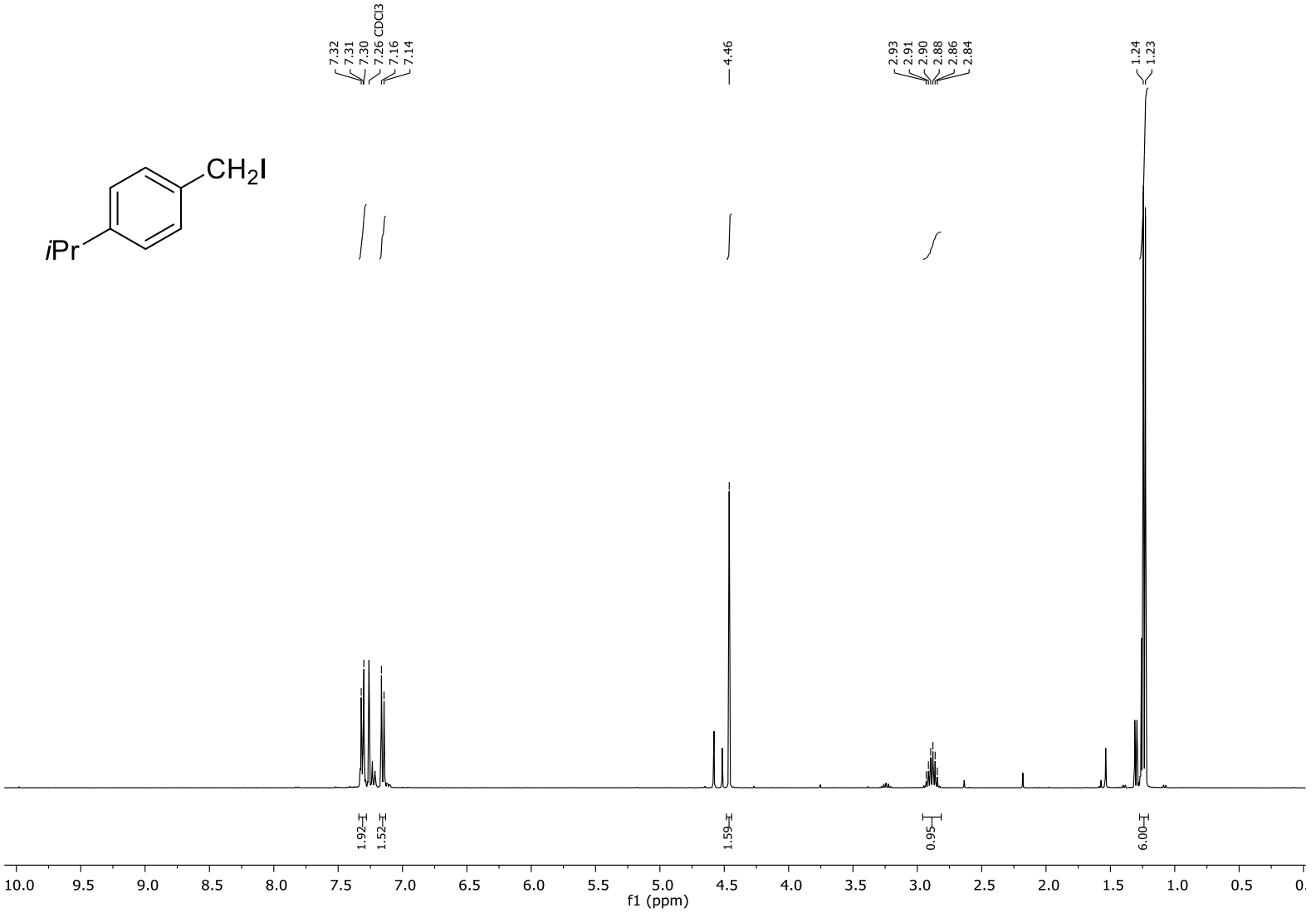

${ }^{13} \mathrm{C}-\mathrm{NMR}$ (100 MHz, $\left.\mathrm{CDCl}_{3}\right)$ :
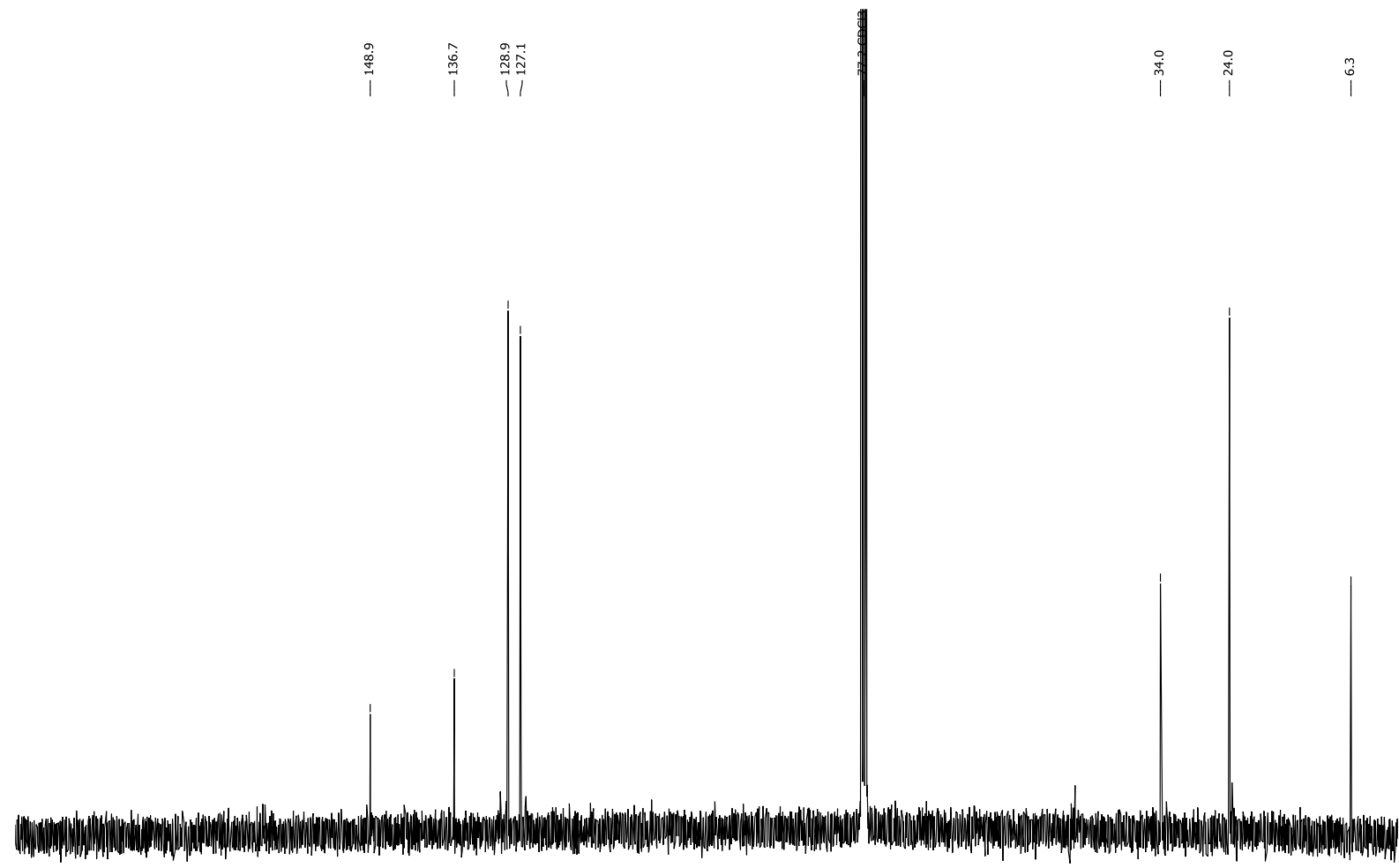

00

$\begin{array}{lllll}190 & 180 & 170 & 160 & 150\end{array}$

$130 \quad 12$

$110 \quad 100$

80

$60 \quad 50$

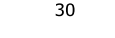




\section{3-(Hydroxymethyl)phenol}

${ }^{1} \mathrm{H}-\mathrm{NMR}$ (400 MHz, $\mathrm{CDCl}_{3}$ ):

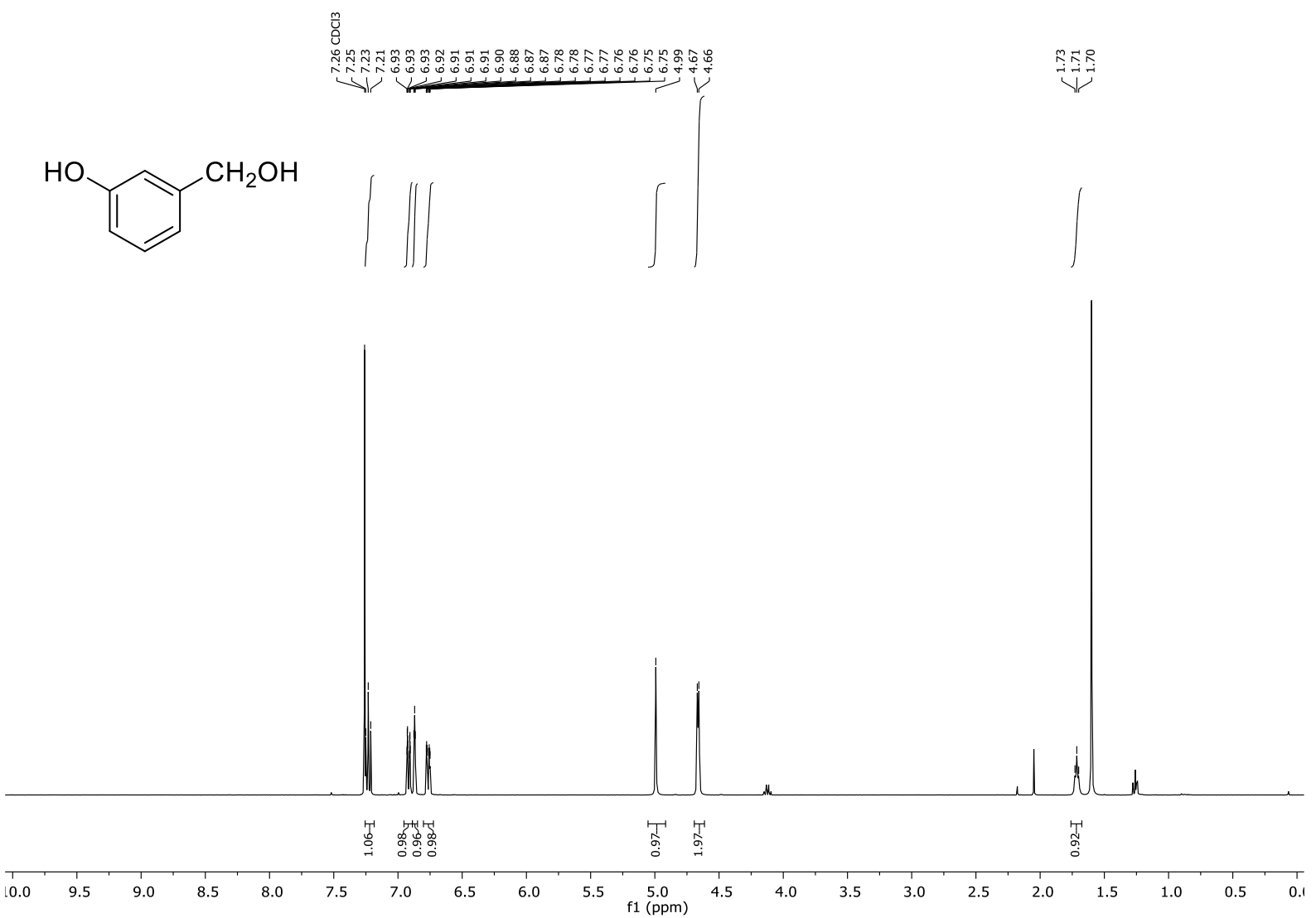

${ }^{13} \mathrm{C}-\mathrm{NMR}$ (100 MHz, $\left.\mathrm{CDCl}_{3}\right)$ :

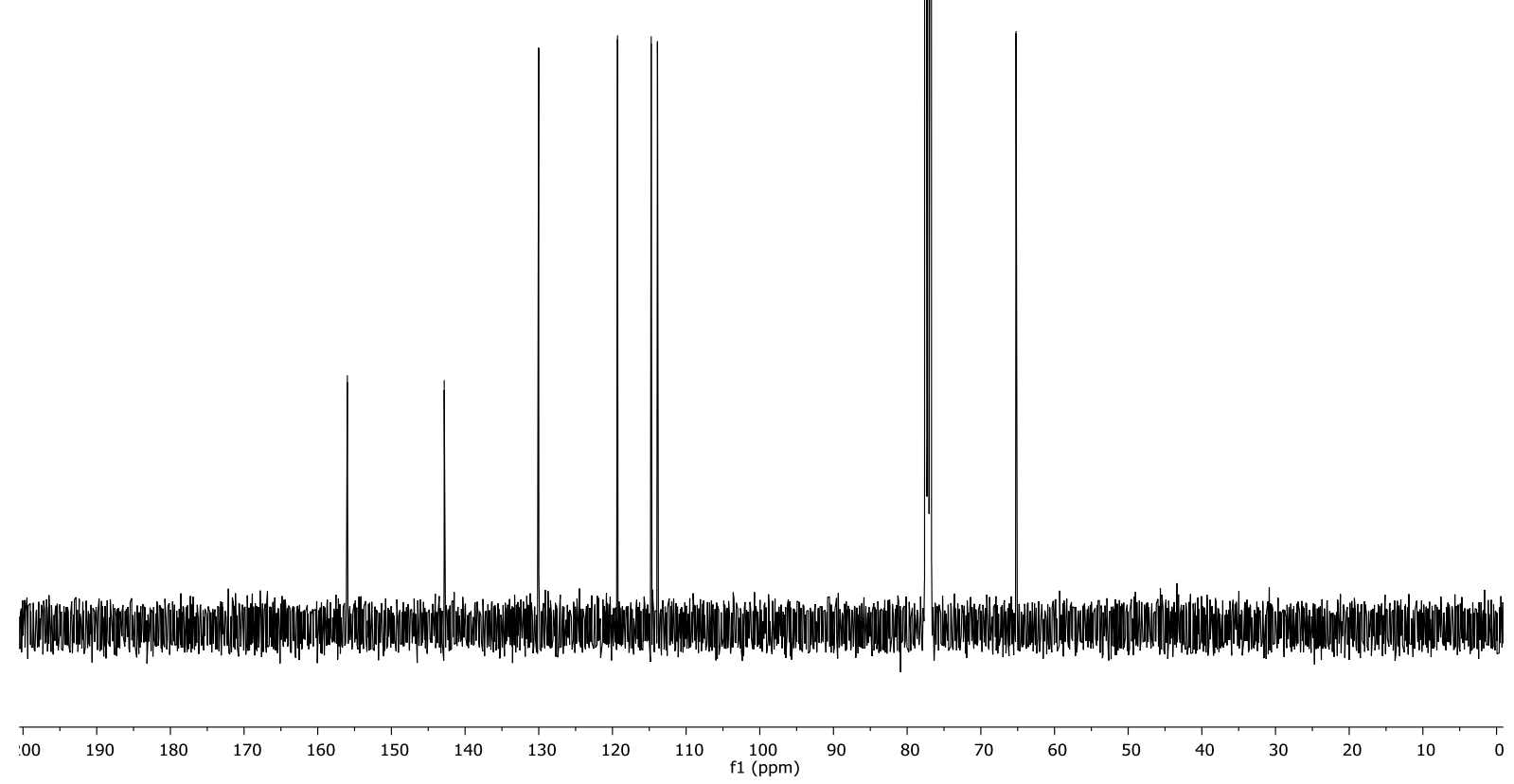




\section{3-(Chloromethyl)phenol}

${ }^{1} \mathrm{H}-\mathrm{NMR}$ (400 MHz, $\mathrm{CDCl}_{3}$ ):

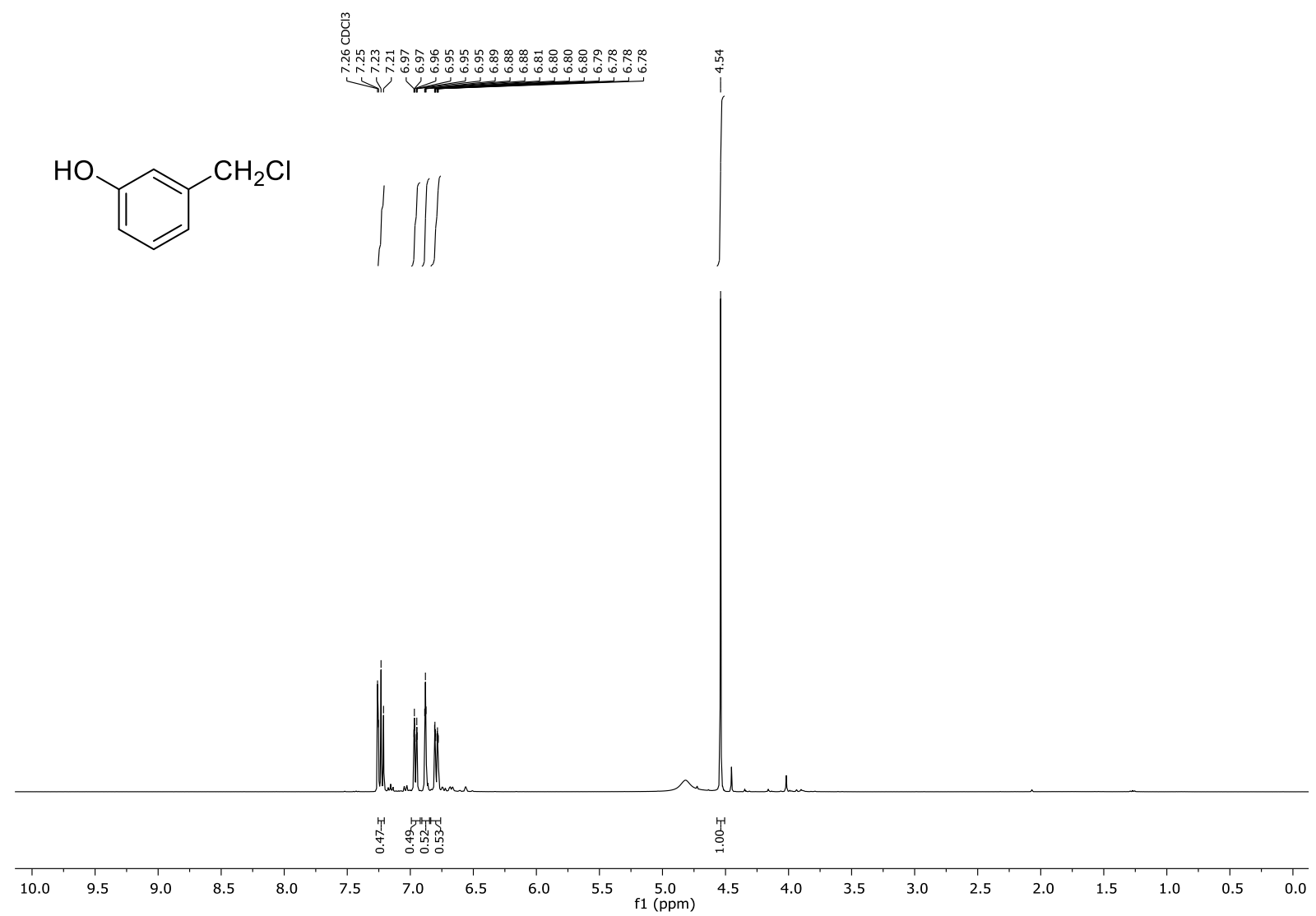

${ }^{13} \mathrm{C}-\mathrm{NMR}\left(100 \mathrm{MHz}, \mathrm{CDCl}_{3}\right)$ :

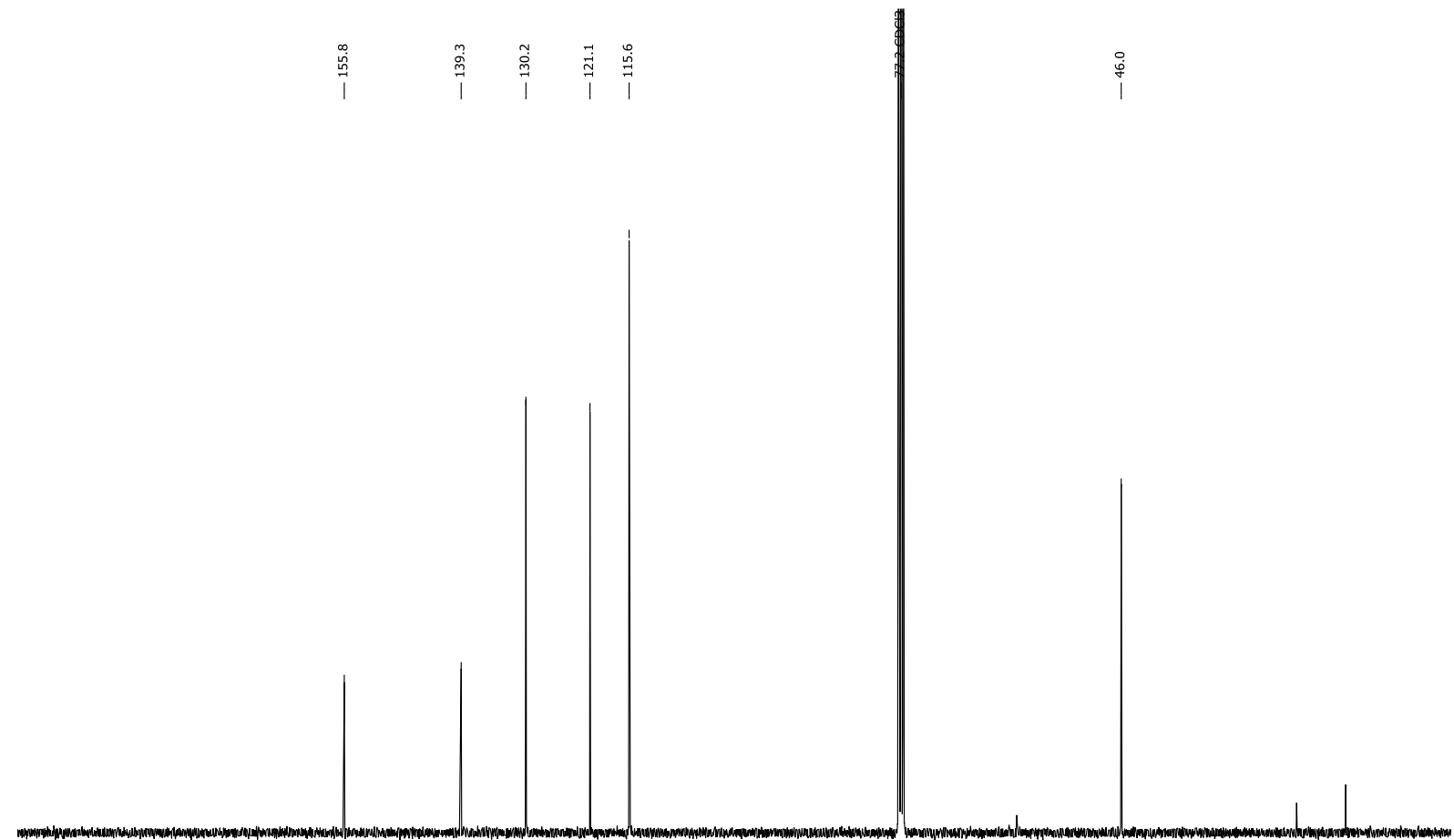

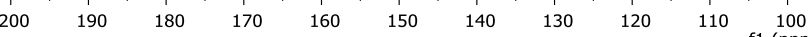

(ppm)

$\begin{array}{lllllllll}80 & 70 & 60 & 50 & 40 & 30 & 20 & 10 & 1\end{array}$ 
Tert-butyl(3-(chloromethyl)phenoxy)dimethylsilane

${ }^{1} \mathrm{H}-\mathrm{NMR}$ (400 MHz, $\mathrm{CDCl}_{3}$ ):

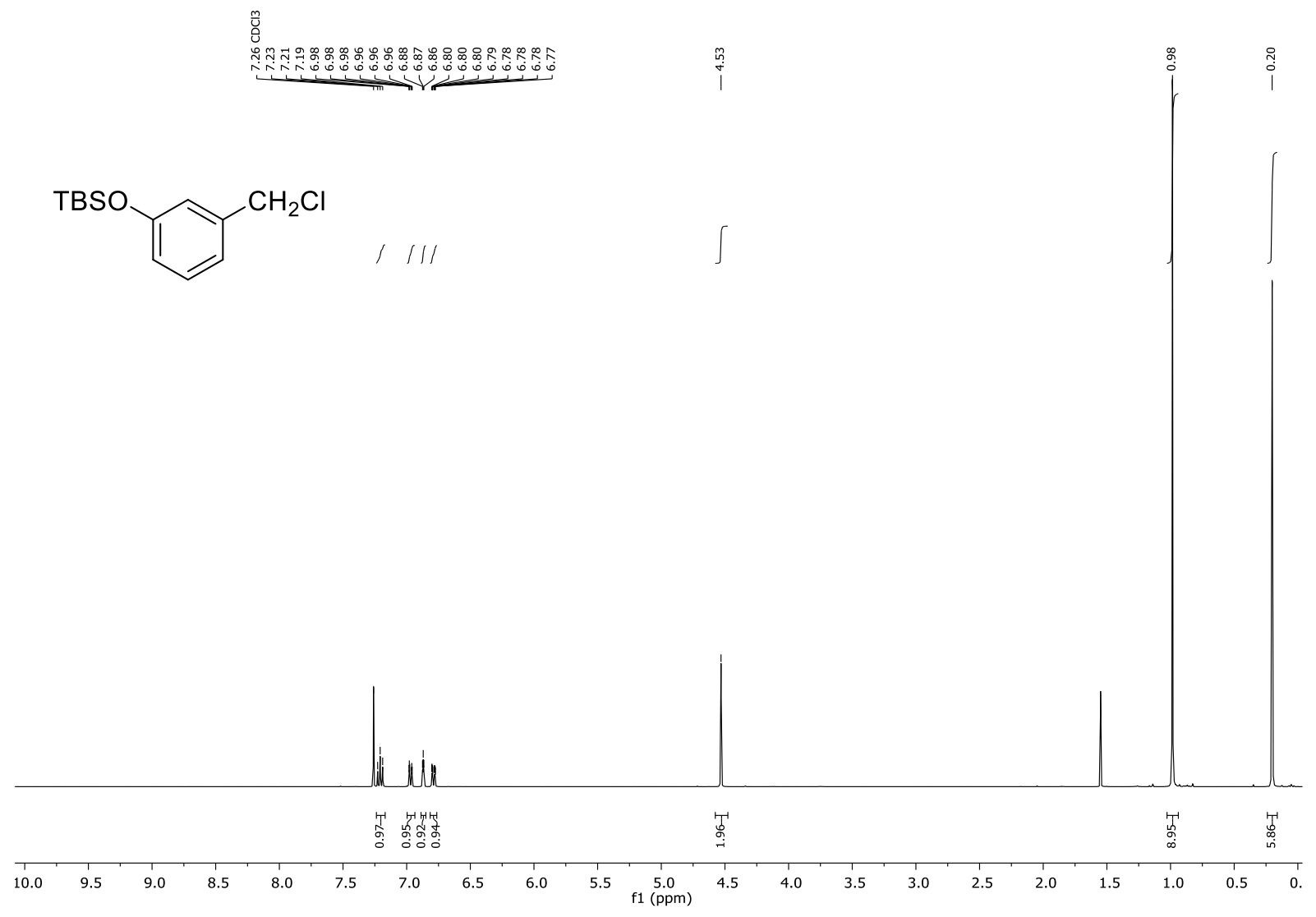

${ }^{13} \mathrm{C}-\mathrm{NMR}$ (100 MHz, $\left.\mathrm{CDCl}_{3}\right)$ :

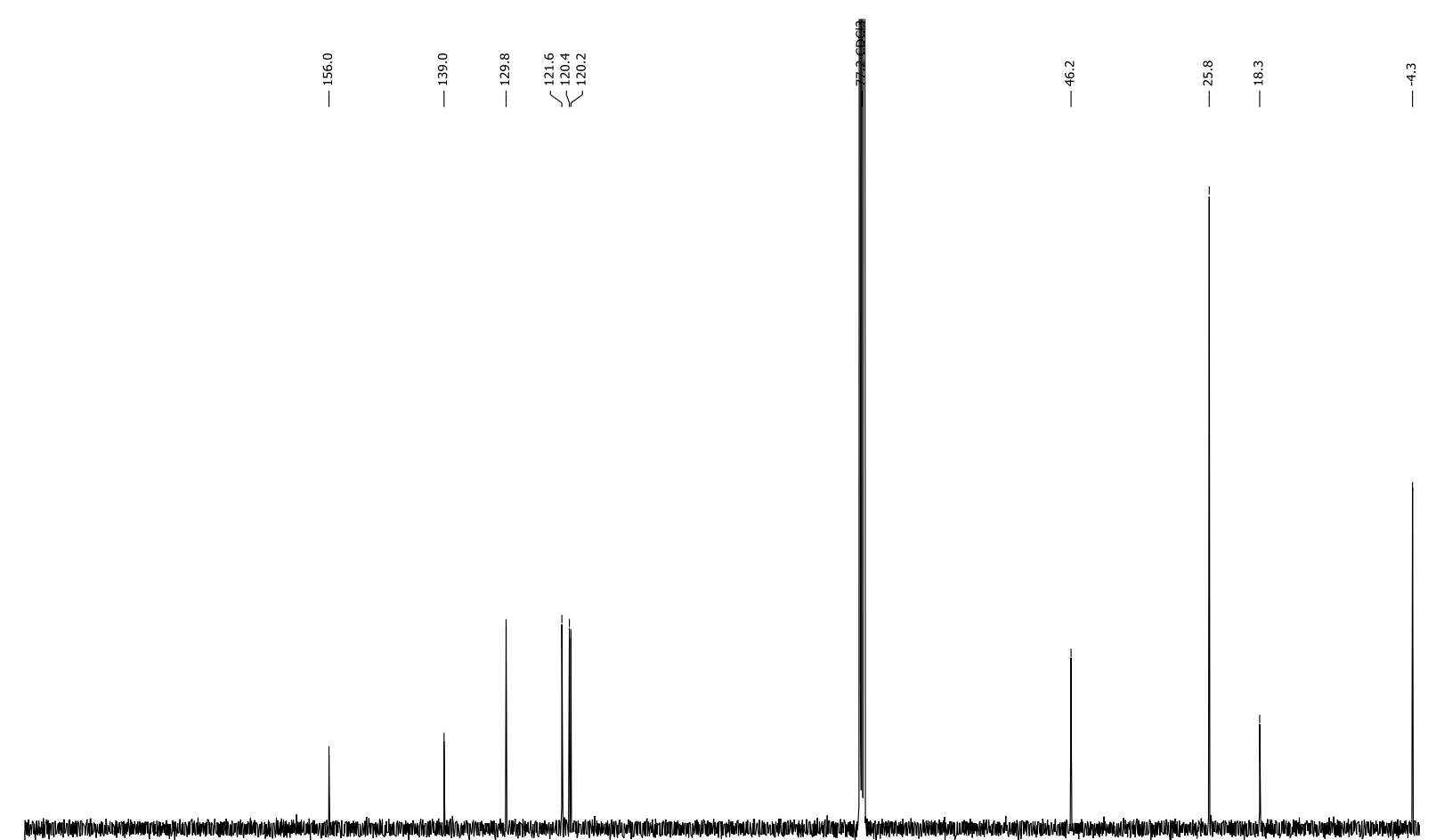

200

$\begin{array}{llllll}190 & 180 & 170 & 160 & 150 & 140\end{array}$

10090

$60 \quad 50$ . 
Tert-butyl(3-(iodomethyl)phenoxy)dimethylsilane (5a)

${ }^{1} \mathrm{H}-\mathrm{NMR}$ (400 MHz, $\mathrm{CDCl}_{3}$ ):

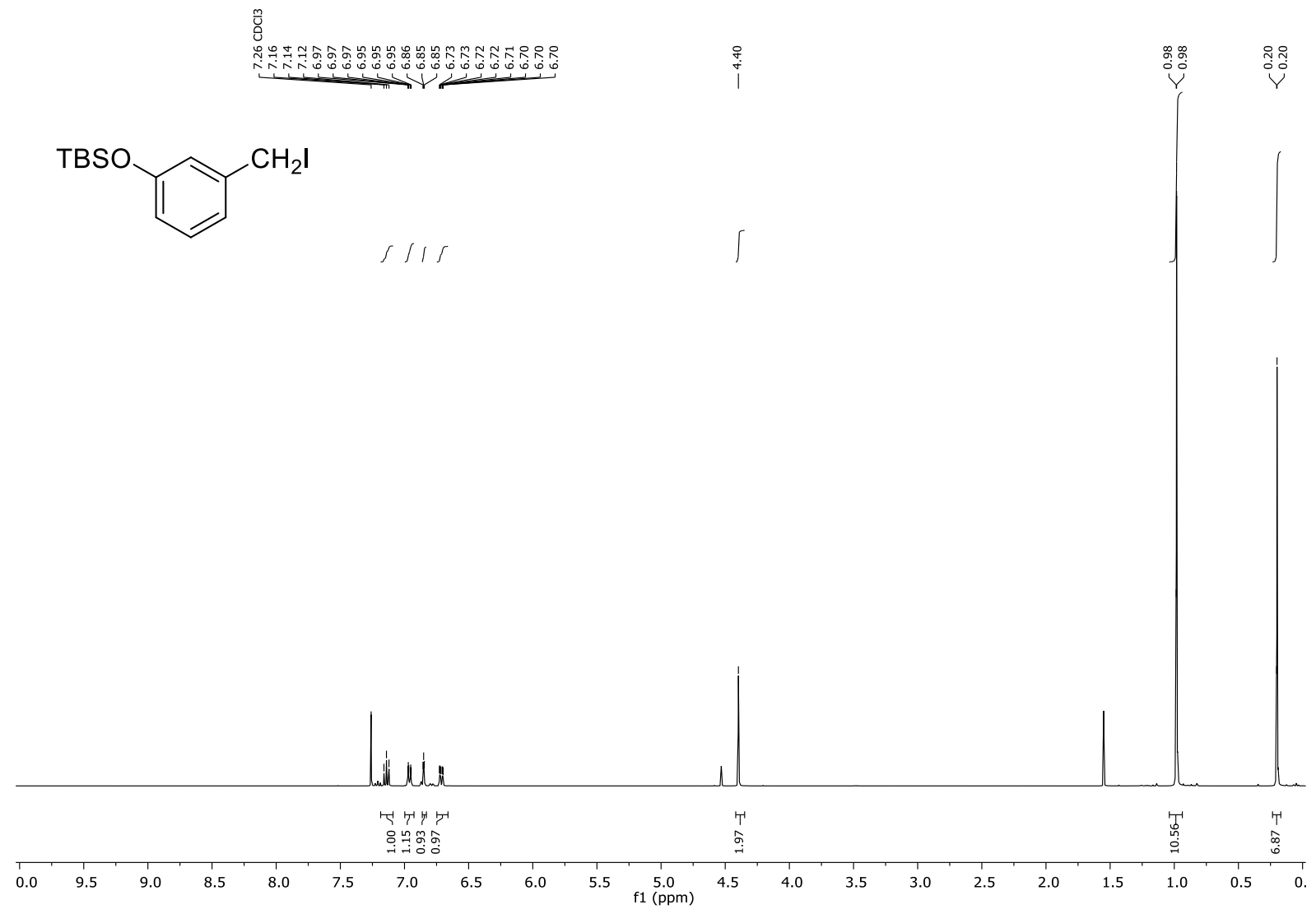

${ }^{13} \mathrm{C}-\mathrm{NMR}$ (100 MHz, $\left.\mathrm{CDCl}_{3}\right)$ :

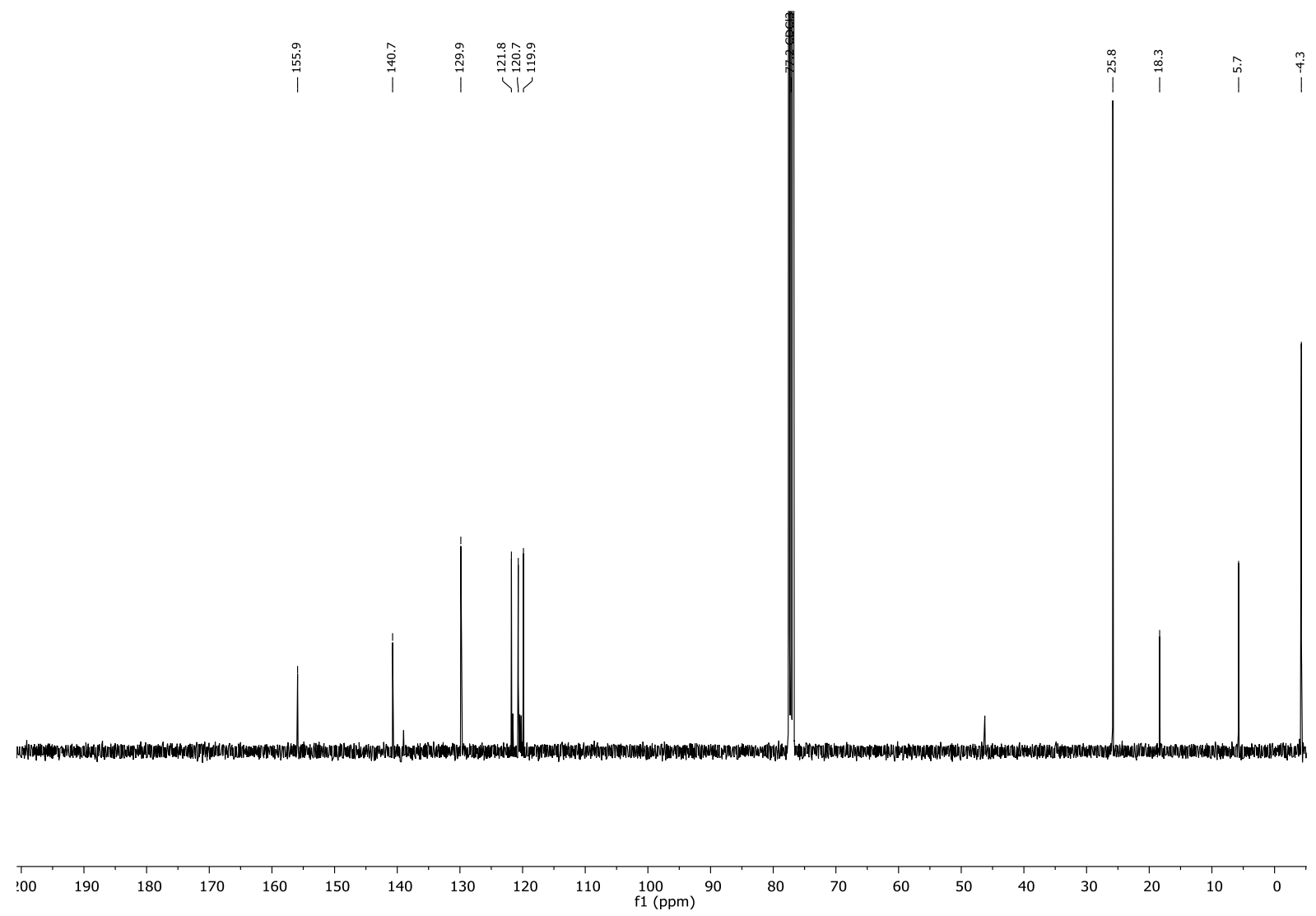


(4-(Iodomethyl)phenyl)(methyl)sulfane (5b)

${ }^{1} \mathrm{H}-\mathrm{NMR}\left(400 \mathrm{MHz}, \mathrm{CDCl}_{3}\right)$ :

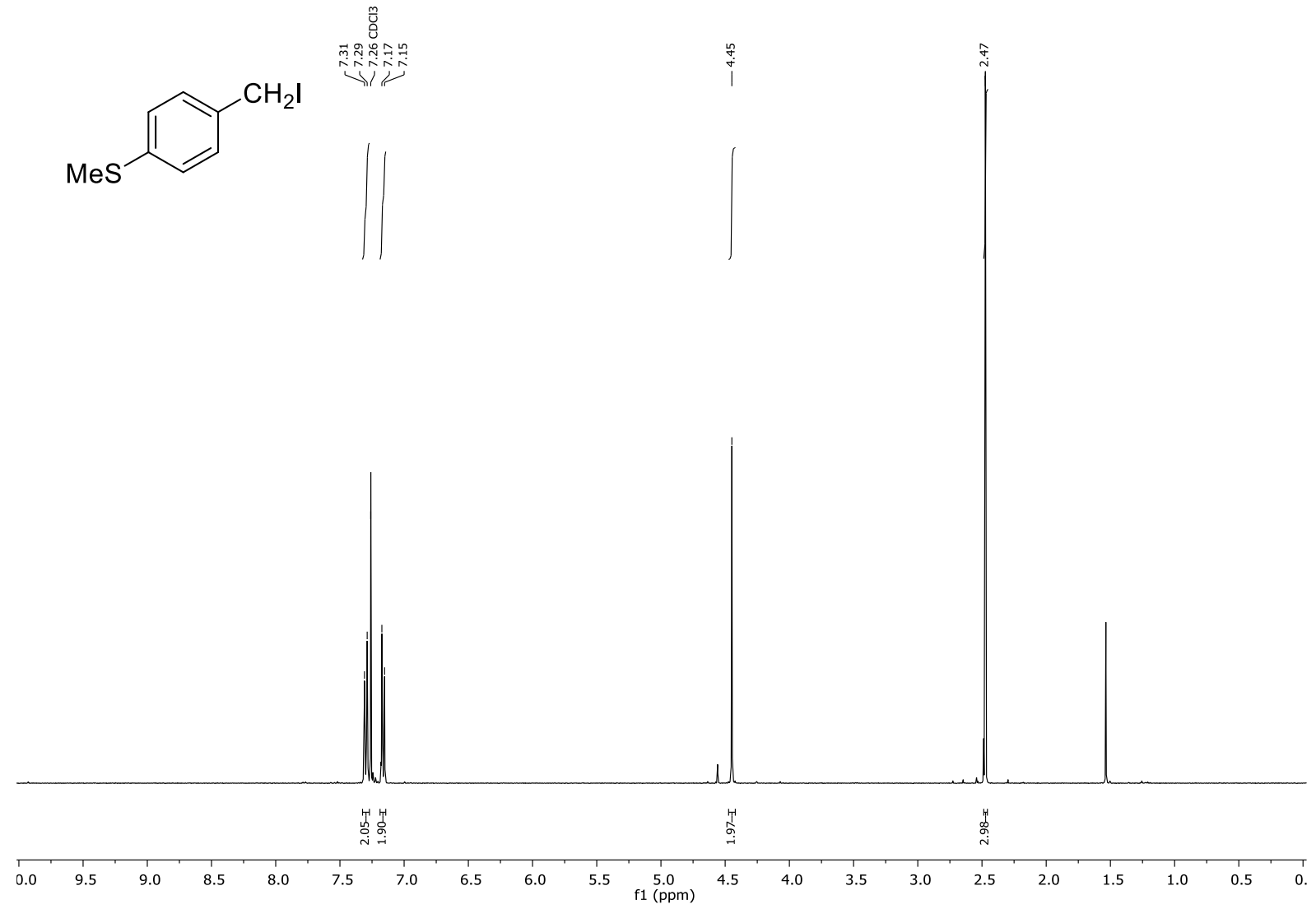

${ }^{13} \mathrm{C}-\mathrm{NMR}$ (100 MHz, $\left.\mathrm{CDCl}_{3}\right)$ :

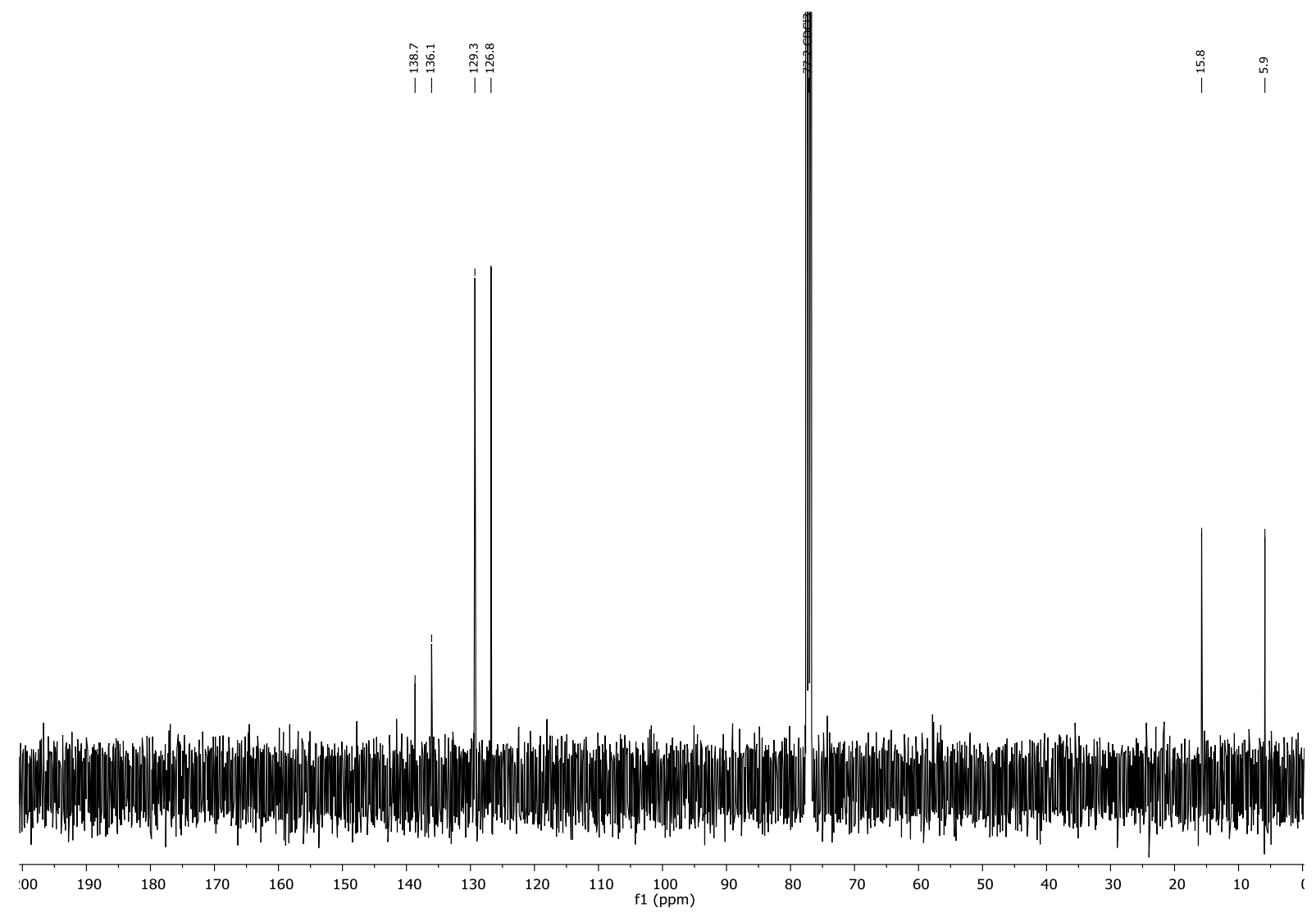


1-(Iodomethyl)-4-methoxybenzene (5c)

${ }^{1} \mathrm{H}-\mathrm{NMR}$ (400 MHz, $\mathrm{CDCl}_{3}$ ):
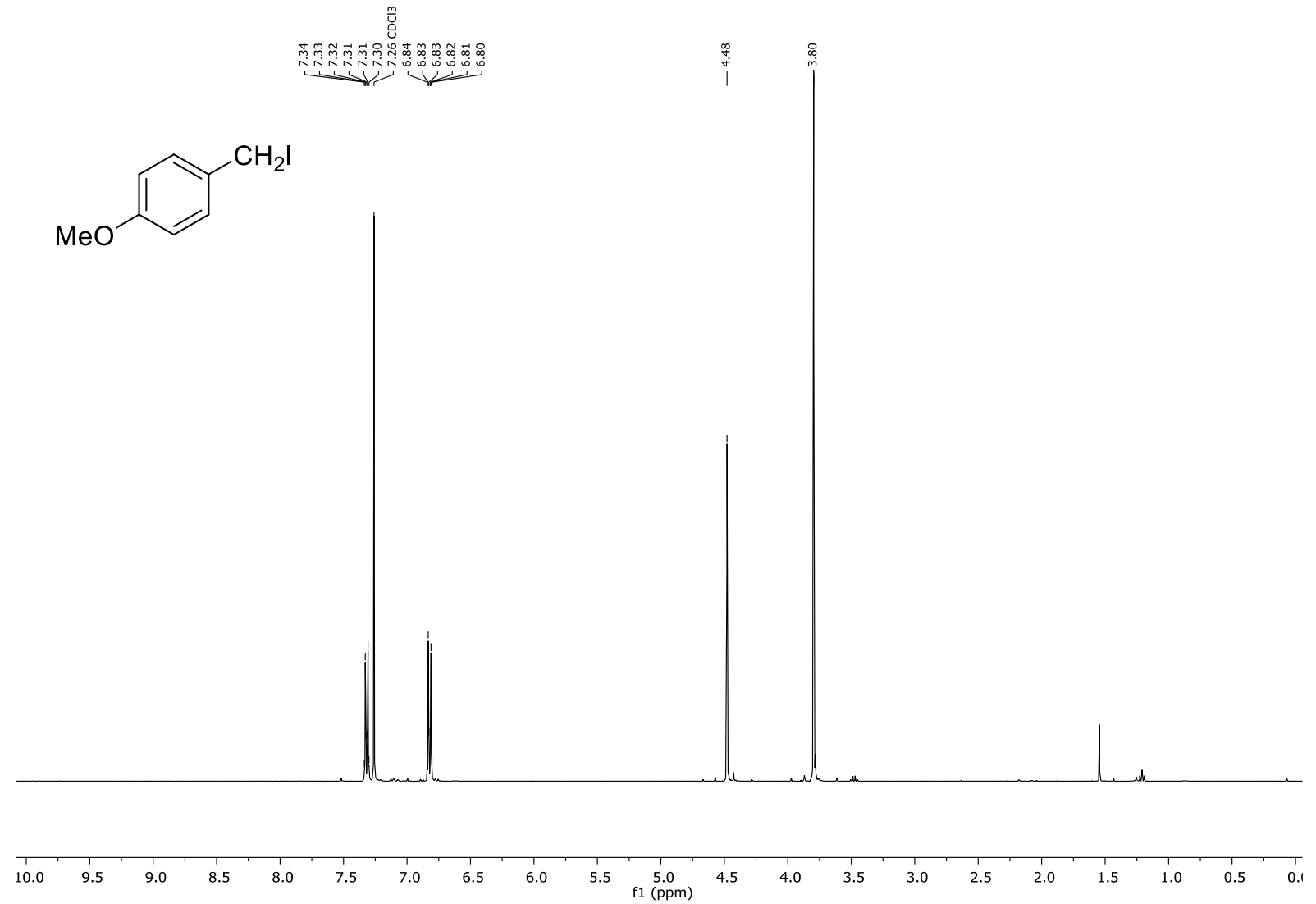

${ }^{13} \mathrm{C}-\mathrm{NMR}\left(100 \mathrm{MHz}, \mathrm{CDCl}_{3}\right)$ :

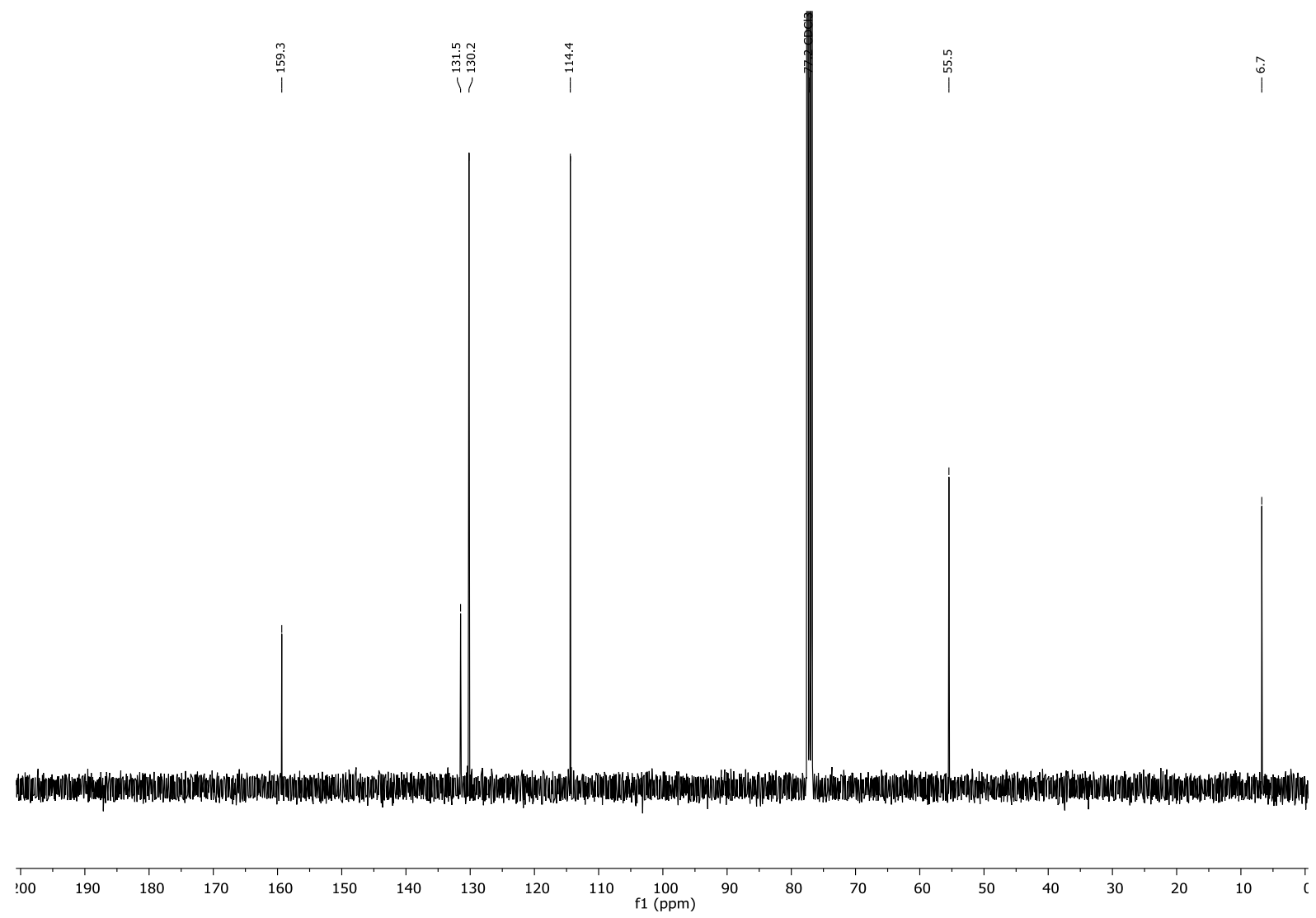


1-(Iodomethyl)-3-methoxybenzene (5d)

${ }^{1} \mathrm{H}-\mathrm{NMR}$ (400 MHz, $\mathrm{CDCl}_{3}$ ):
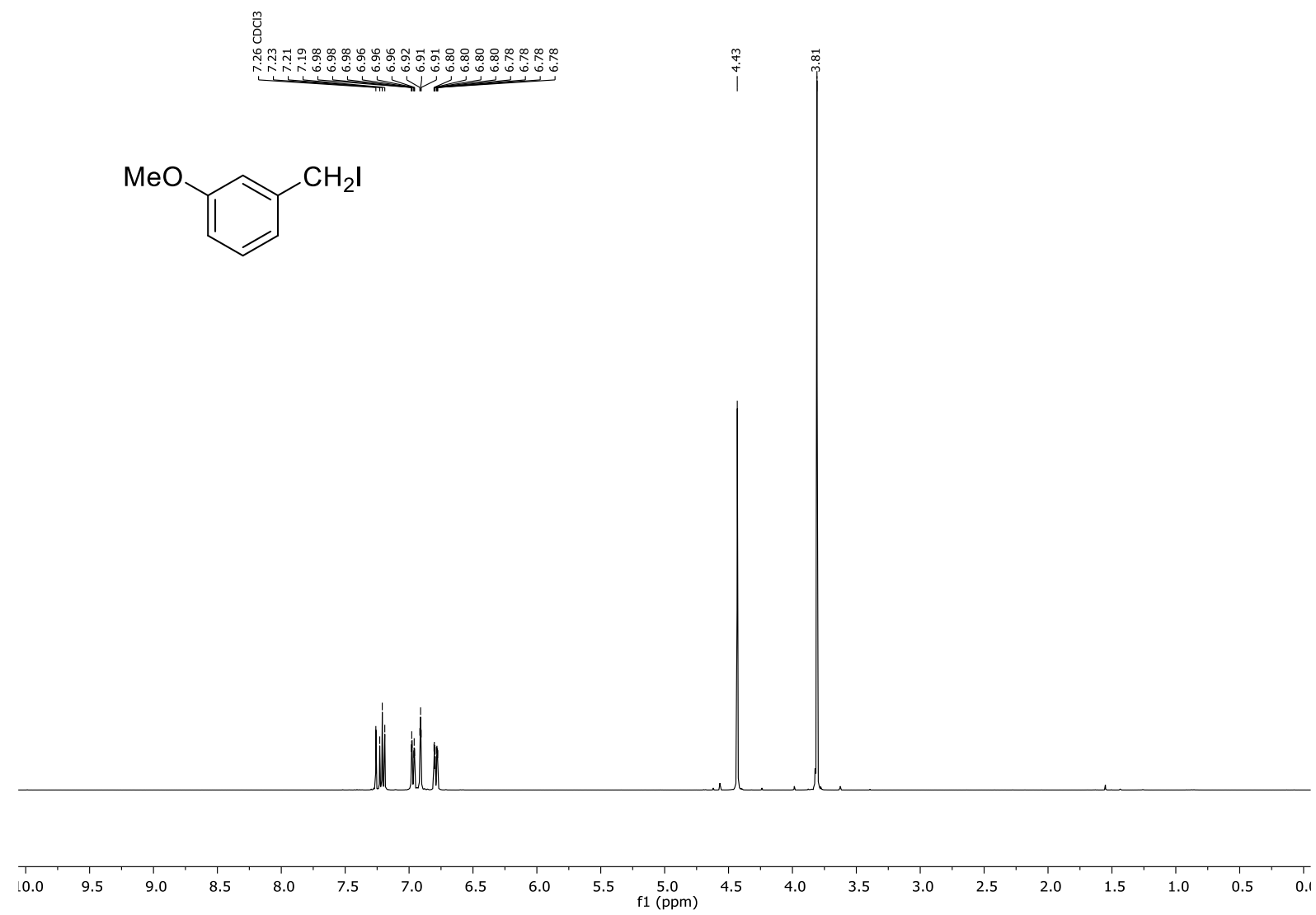

${ }^{13} \mathrm{C}-\mathrm{NMR}$ (100 MHz, $\left.\mathrm{CDCl}_{3}\right)$ :

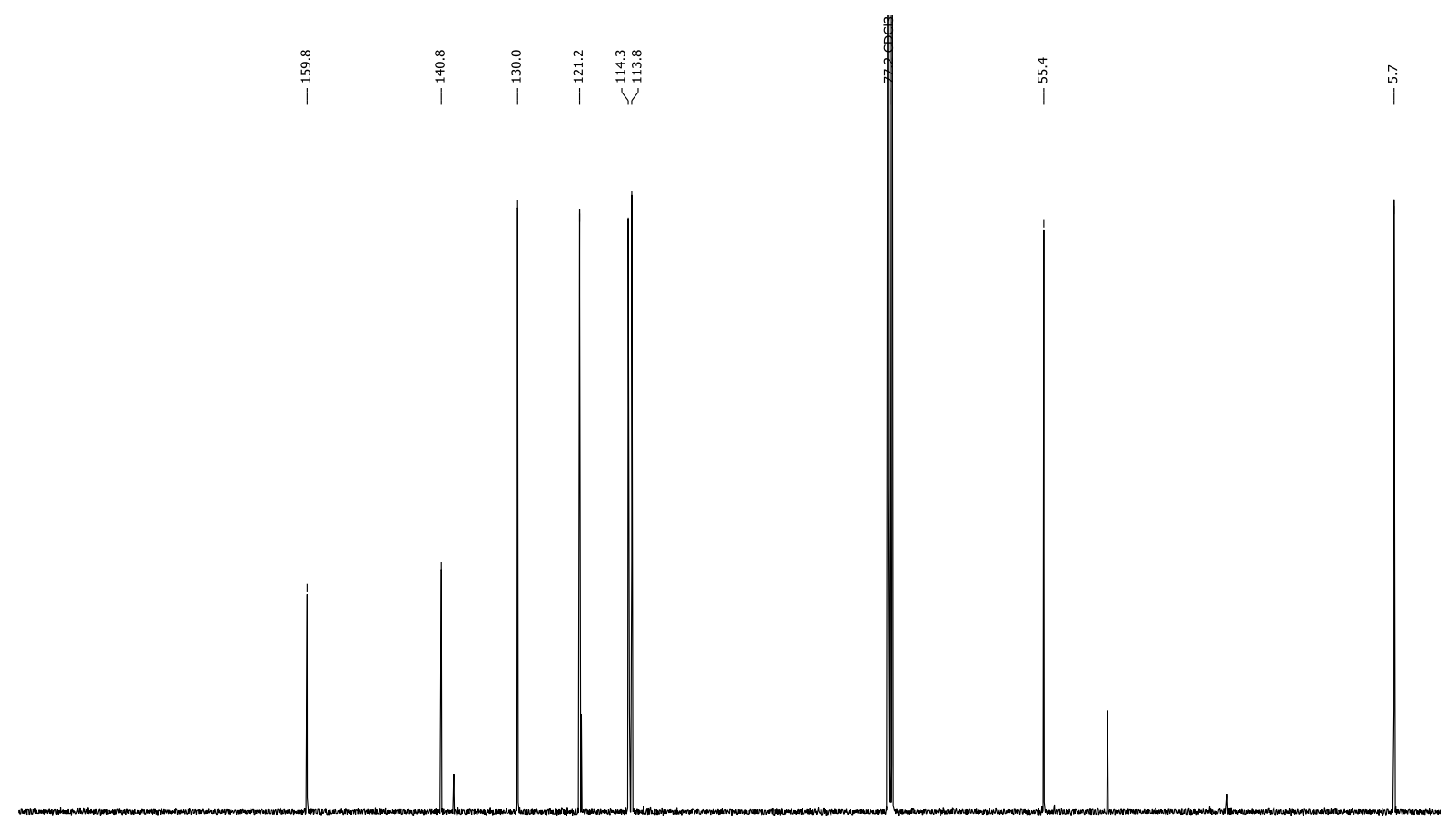


1-Fluoro-2-(iodomethyl)benzene (8a)

${ }^{1} \mathrm{H}-\mathrm{NMR}$ (400 MHz, $\mathrm{CDCl}_{3}$ ):

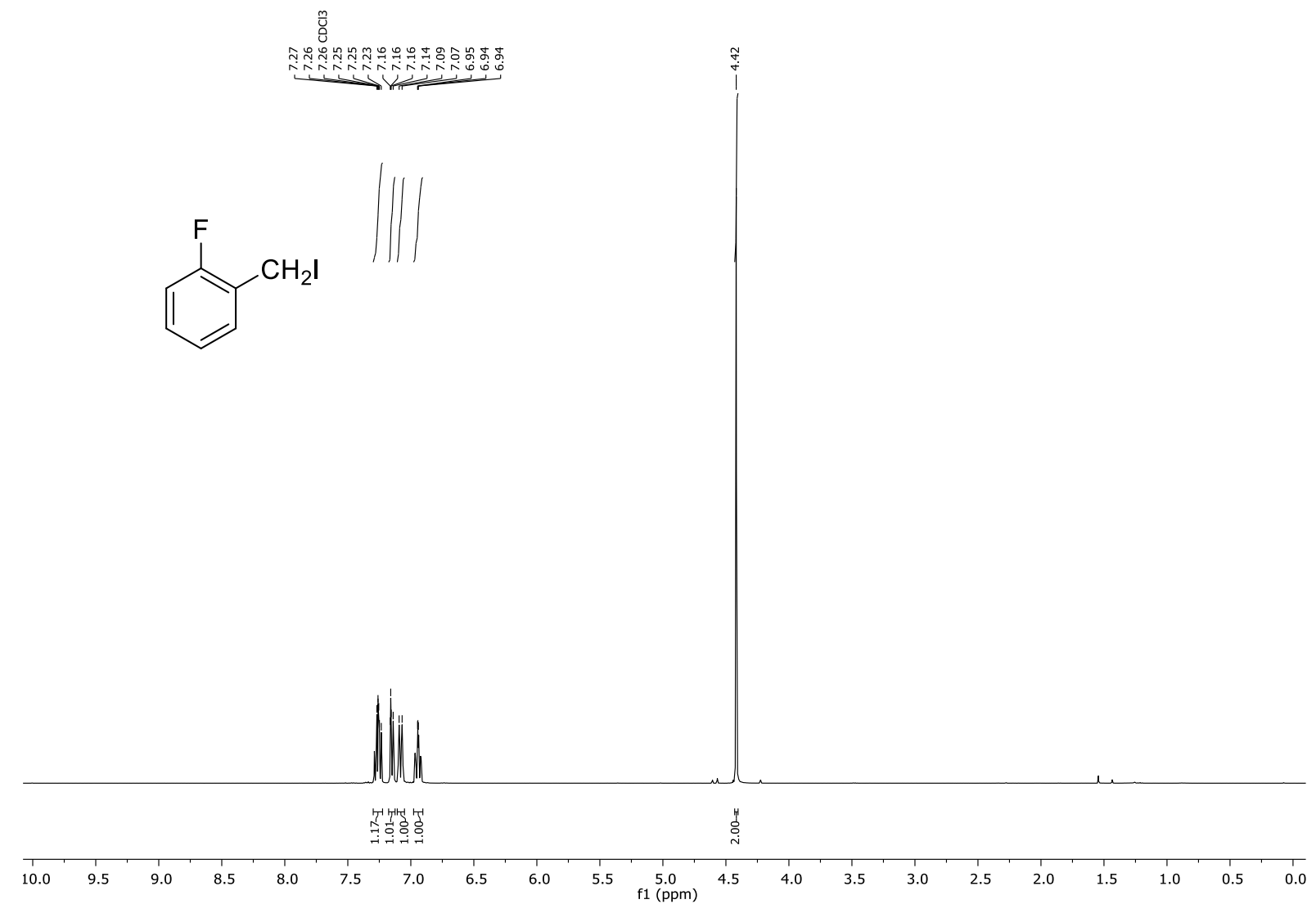

${ }^{13} \mathrm{C}-\mathrm{NMR}$ (100 MHz, $\left.\mathrm{CDCl}_{3}\right)$ :

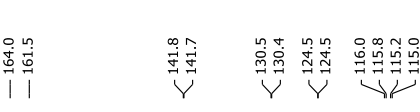

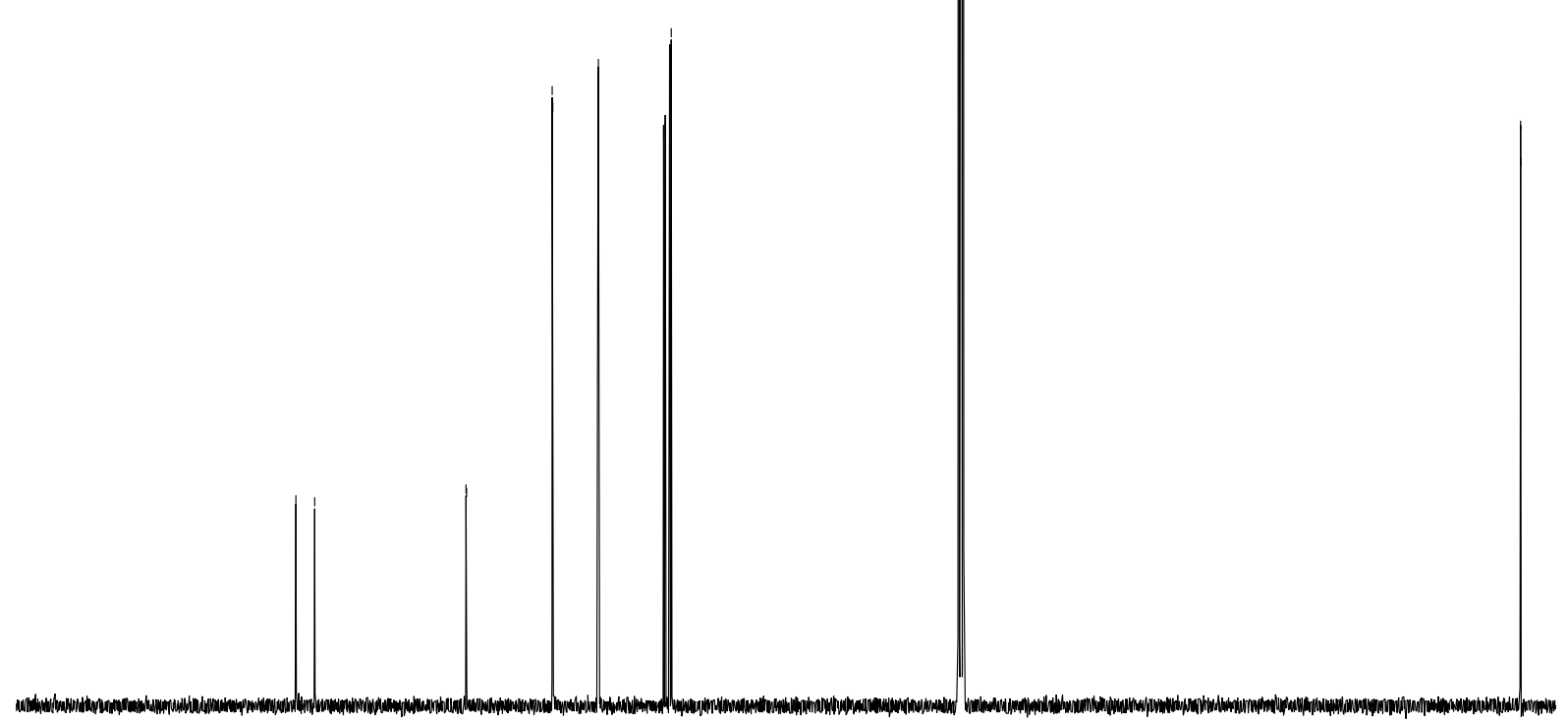

00

$\begin{array}{lllll}190 & 180 & 170 & 160 & 150\end{array}$

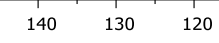

$110 \quad 100$

80

60

$40 \quad 30$

$20 \quad 10 \quad c$ 
1-Chloro-2-(iodomethyl)benzene (8b)

${ }^{1} \mathrm{H}-\mathrm{NMR}$ (400 MHz, $\left.\mathrm{CDCl}_{3}\right)$ :

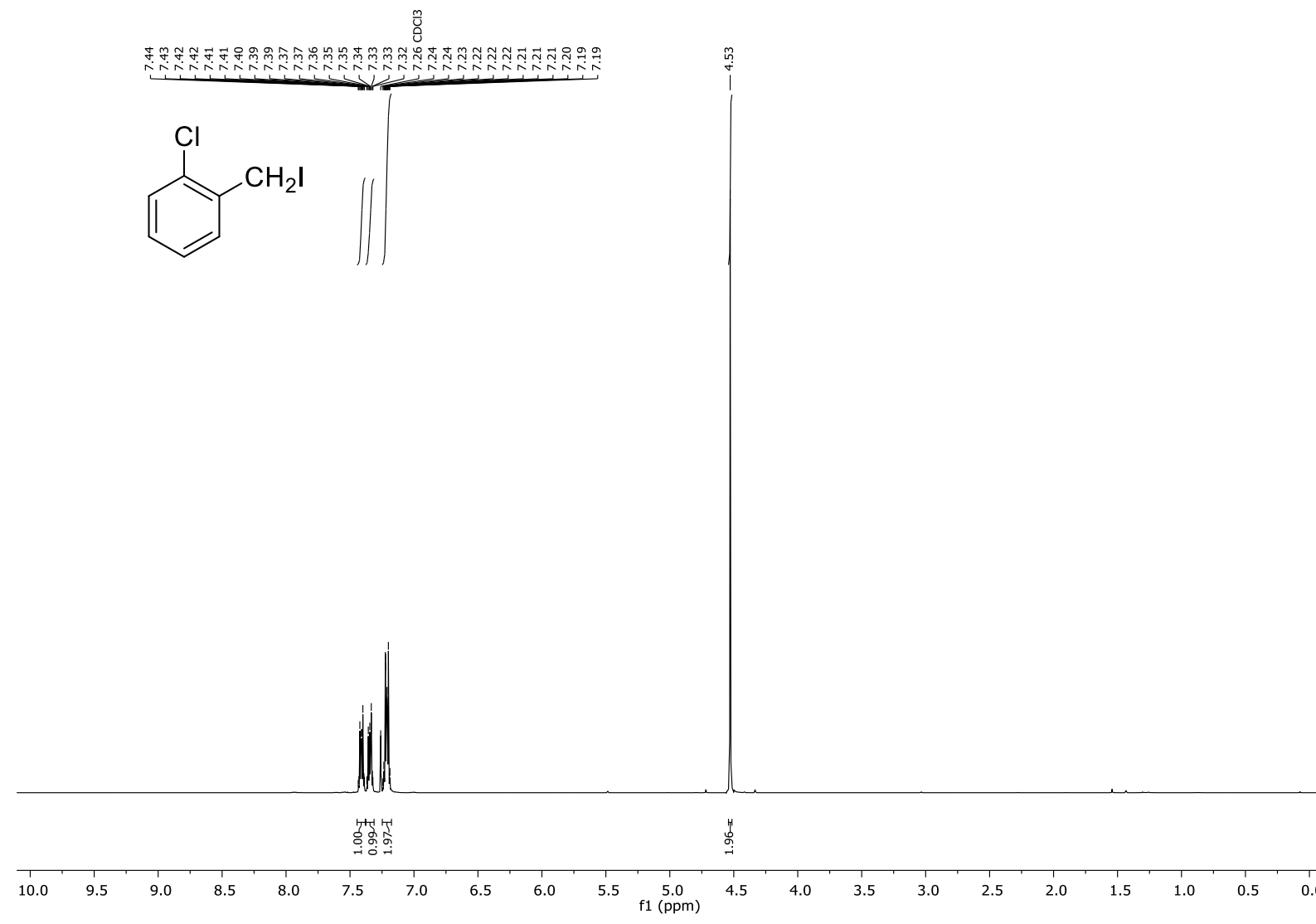

${ }^{13} \mathrm{C}-\mathrm{NMR}$ (100 MHz, $\left.\mathrm{CDCl}_{3}\right)$ :

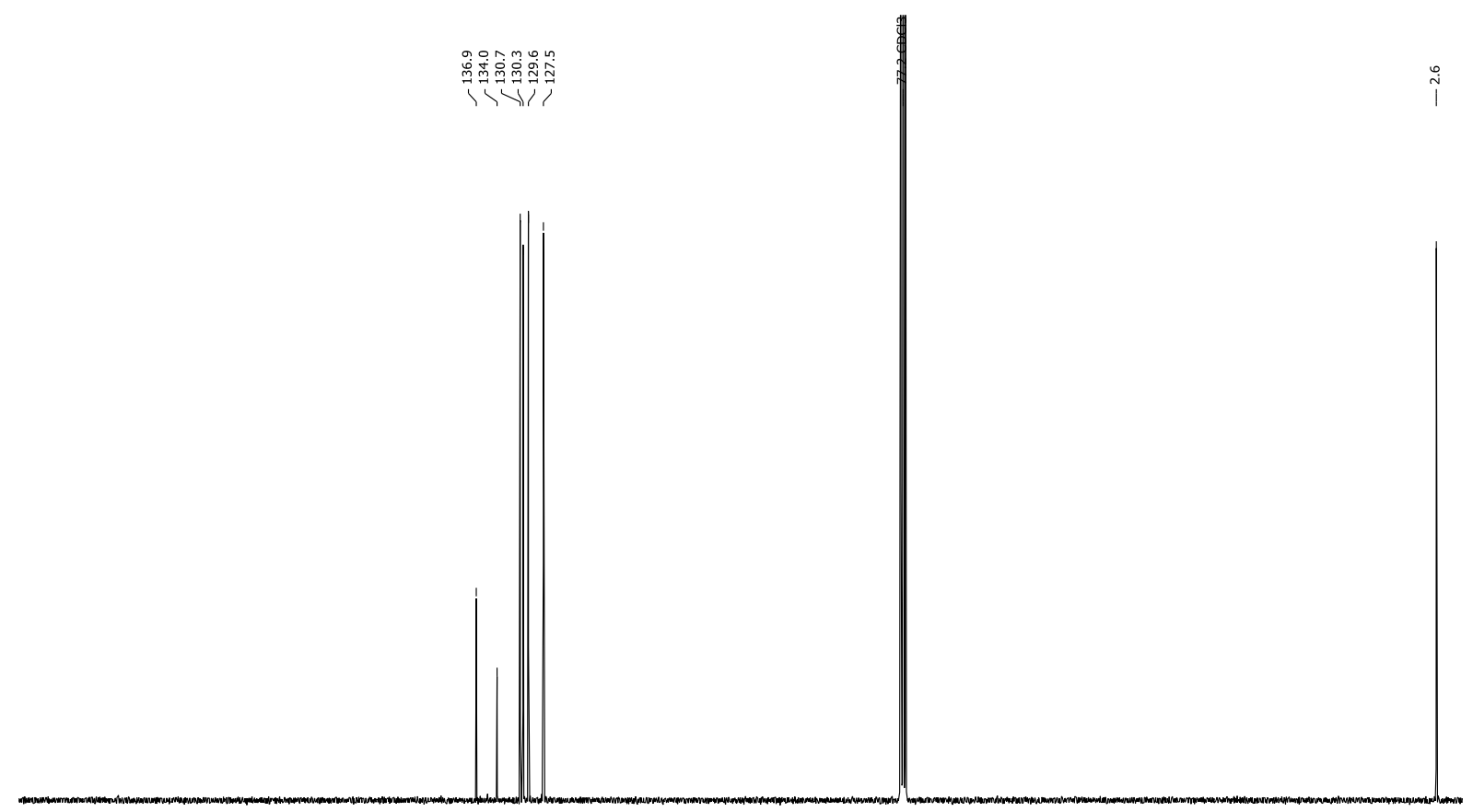


1-(Iodomethyl)-3-(trifluoromethyl)benzene (8c)

${ }^{13} \mathrm{C}-\mathrm{NMR}\left(100 \mathrm{MHz}, \mathrm{CDCl}_{3}\right)$ :

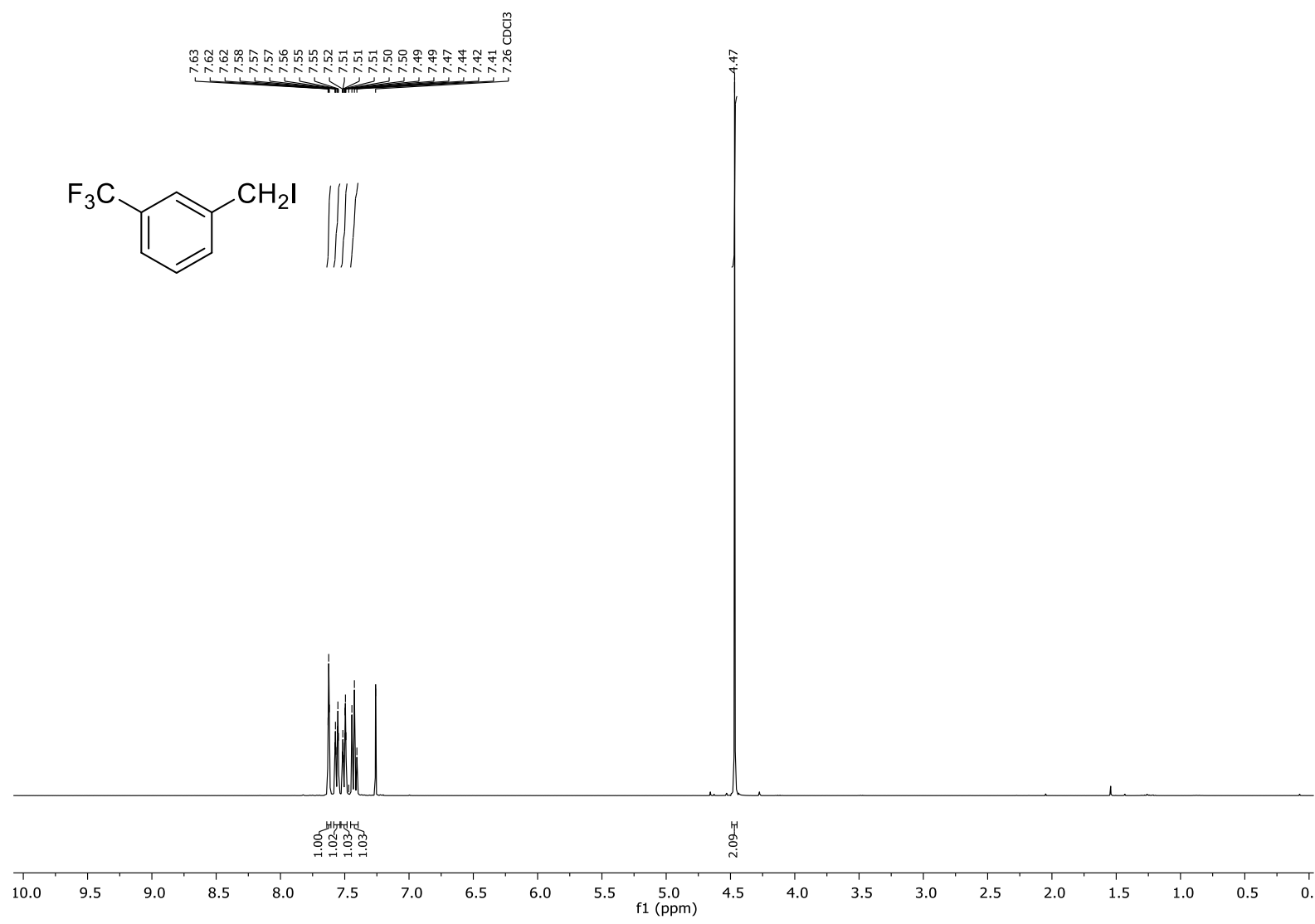

${ }^{13} \mathrm{C}-\mathrm{NMR}\left(100 \mathrm{MHz}, \mathrm{CDCl}_{3}\right)$ :

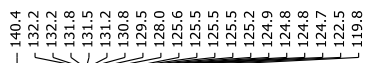

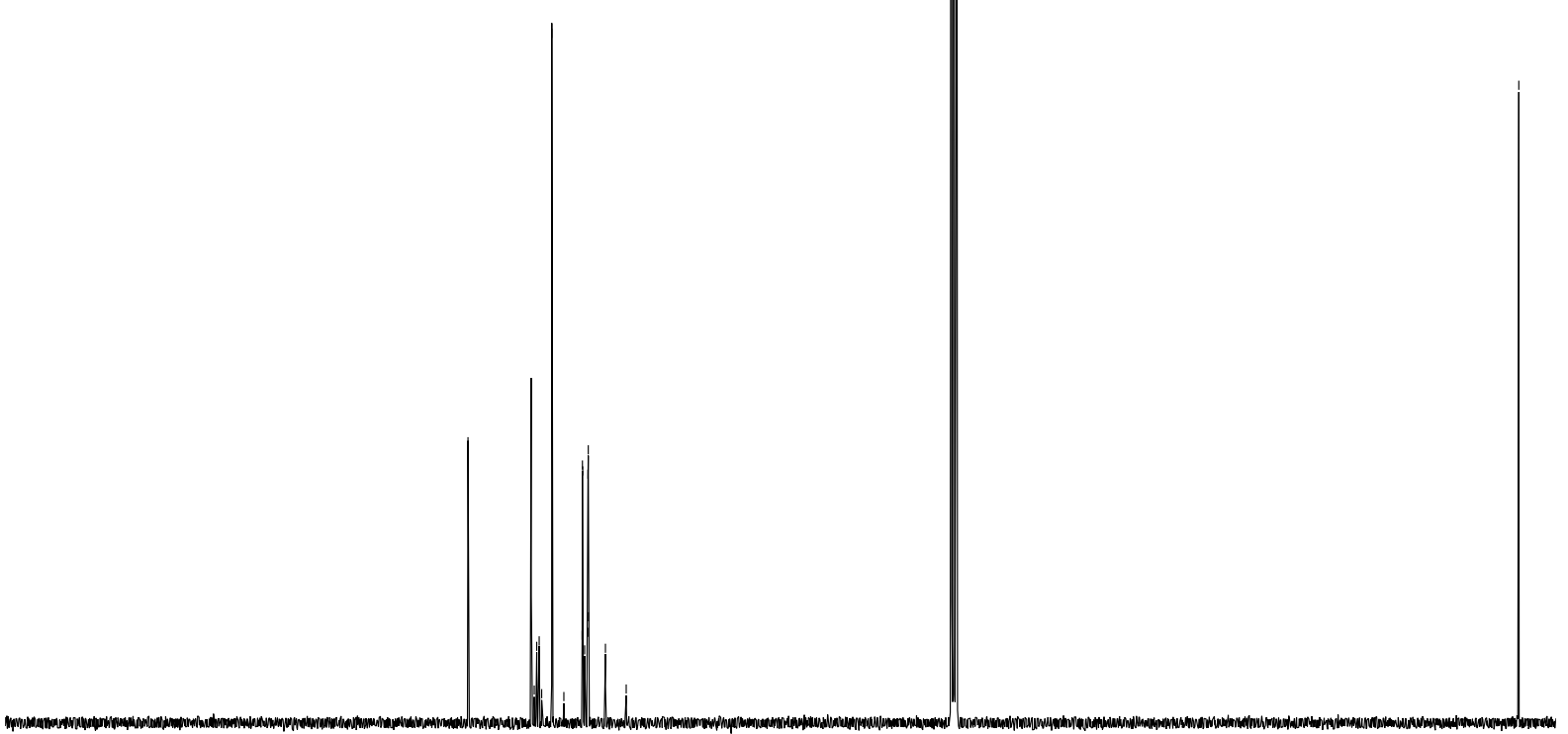

$! 00$

$\begin{array}{lllll}190 & 180 & 170 & 160 & 150\end{array}$

$\begin{array}{lll}140 & 130 & 120\end{array}$

$110 \quad 100$

$90 \quad 80$

$\begin{array}{lll}70 & 60\end{array}$

(1)

30 
2-Chloro-5-(iodomethyl)pyridine (11a)

${ }^{1} \mathrm{H}-\mathrm{NMR}$ (400 MHz, $\mathrm{CDCl}_{3}$ ):

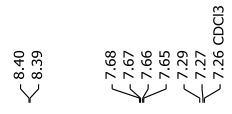<smiles>C=CCCC</smiles><smiles>Clc1ccc(CI)cn1</smiles>

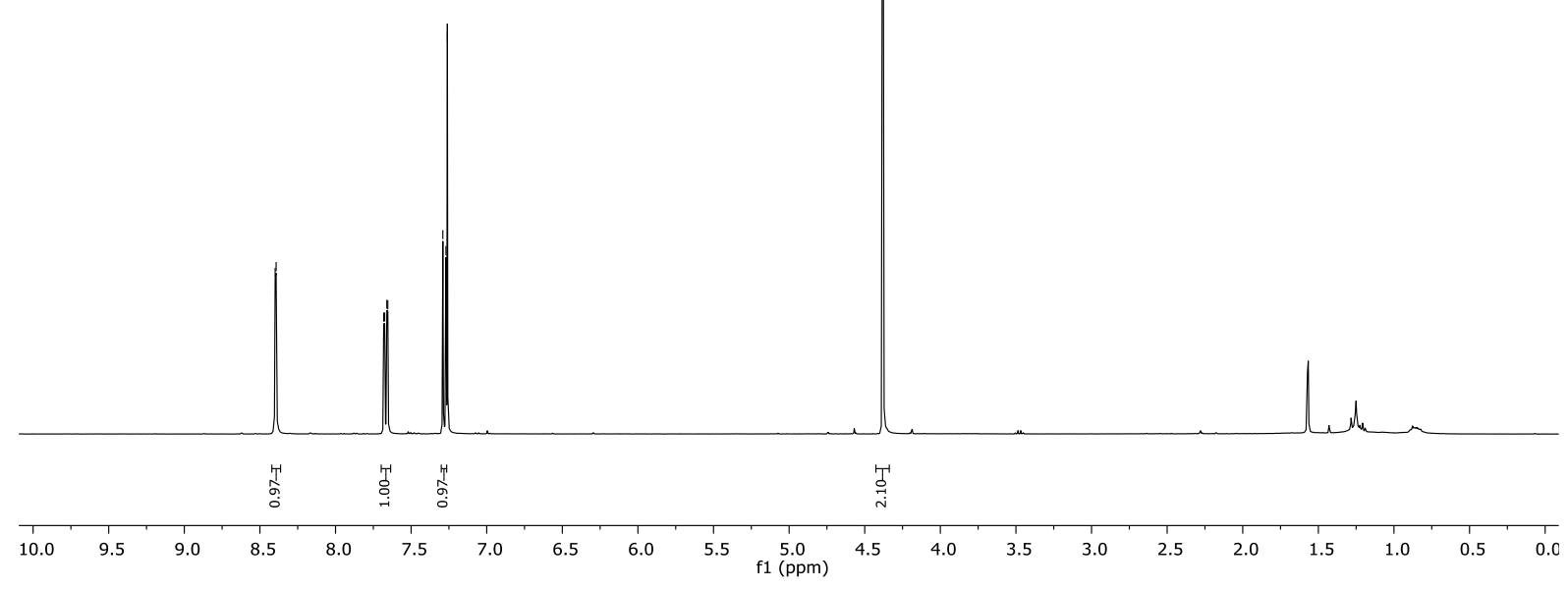

${ }^{13} \mathrm{C}-\mathrm{NMR}\left(100 \mathrm{MHz}, \mathrm{CDCl}_{3}\right)$ :

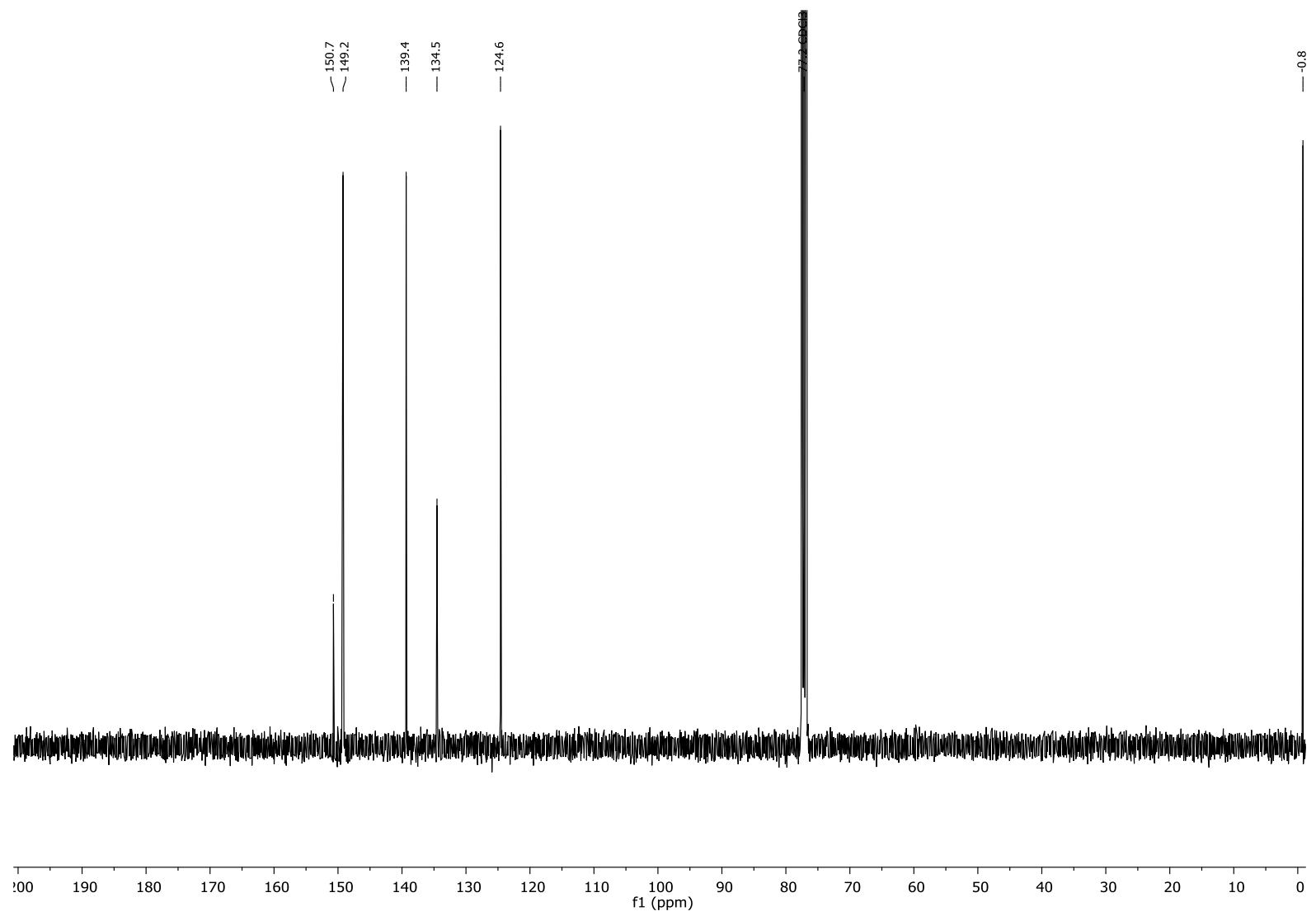




\section{6-Chloro-2-fluoro-3-iodopyridine}

${ }^{1} \mathrm{H}-\mathrm{NMR}$ (400 MHz, $\mathrm{CDCl}_{3}$ ):
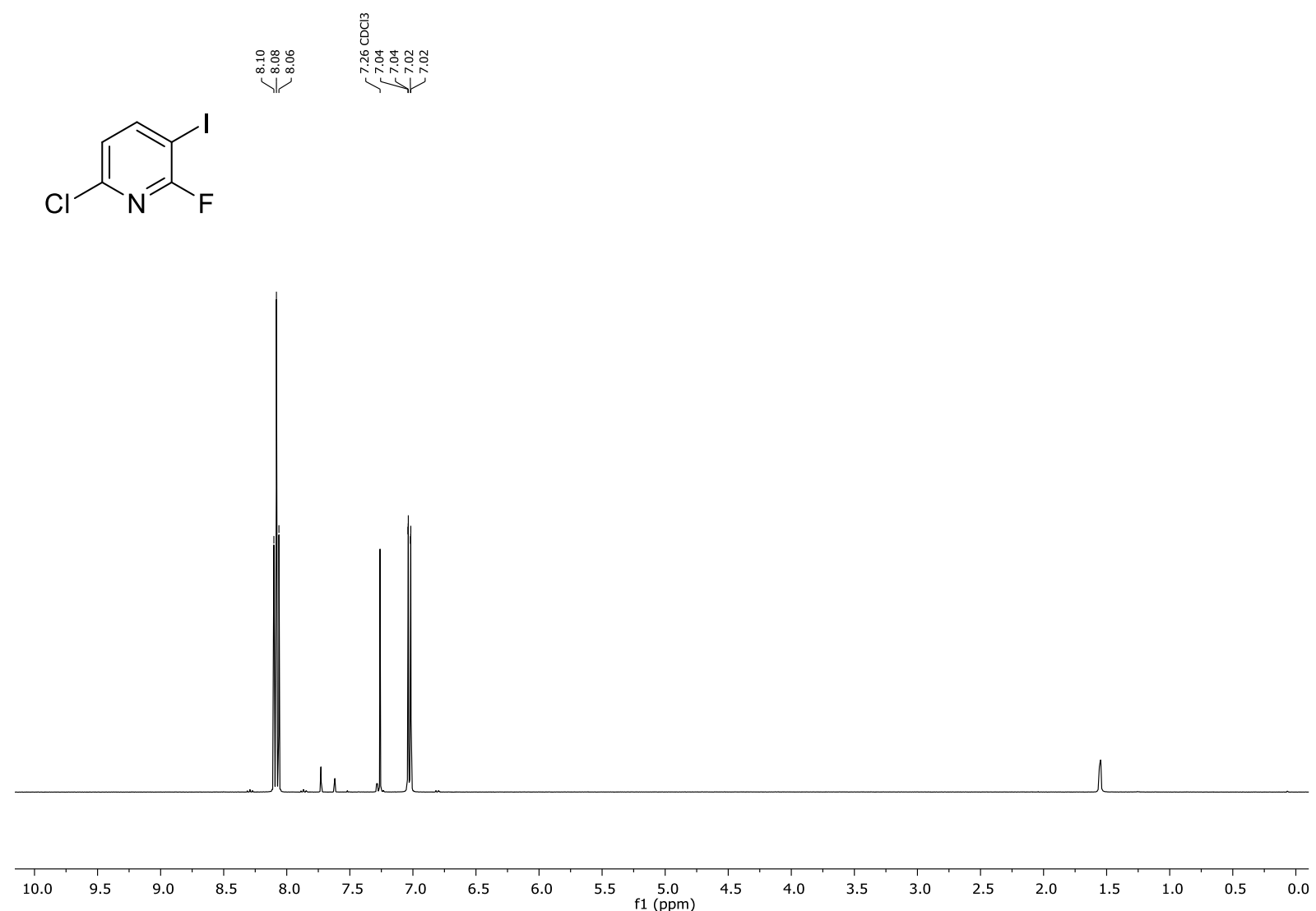

${ }^{13} \mathrm{C}-\mathrm{NMR}\left(100 \mathrm{MHz}, \mathrm{CDCl}_{3}\right)$ :

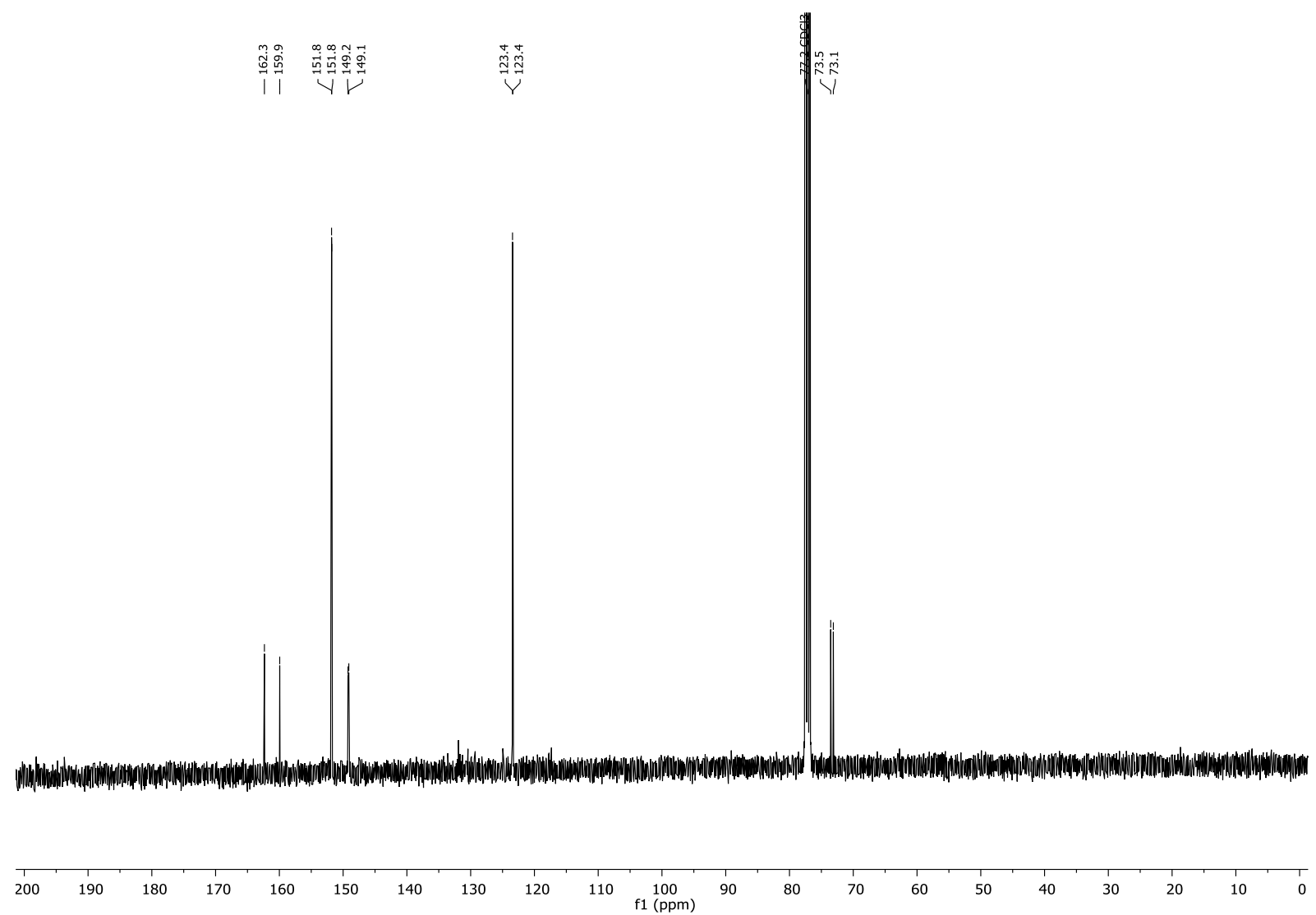


1-(6-Chloro-2-fluoropyridin-3-yl)- $N, N$-dimethylmethanamine

${ }^{1} \mathrm{H}-\mathrm{NMR}$ (400 MHz, $\left.\mathrm{CDCl}_{3}\right)$ :

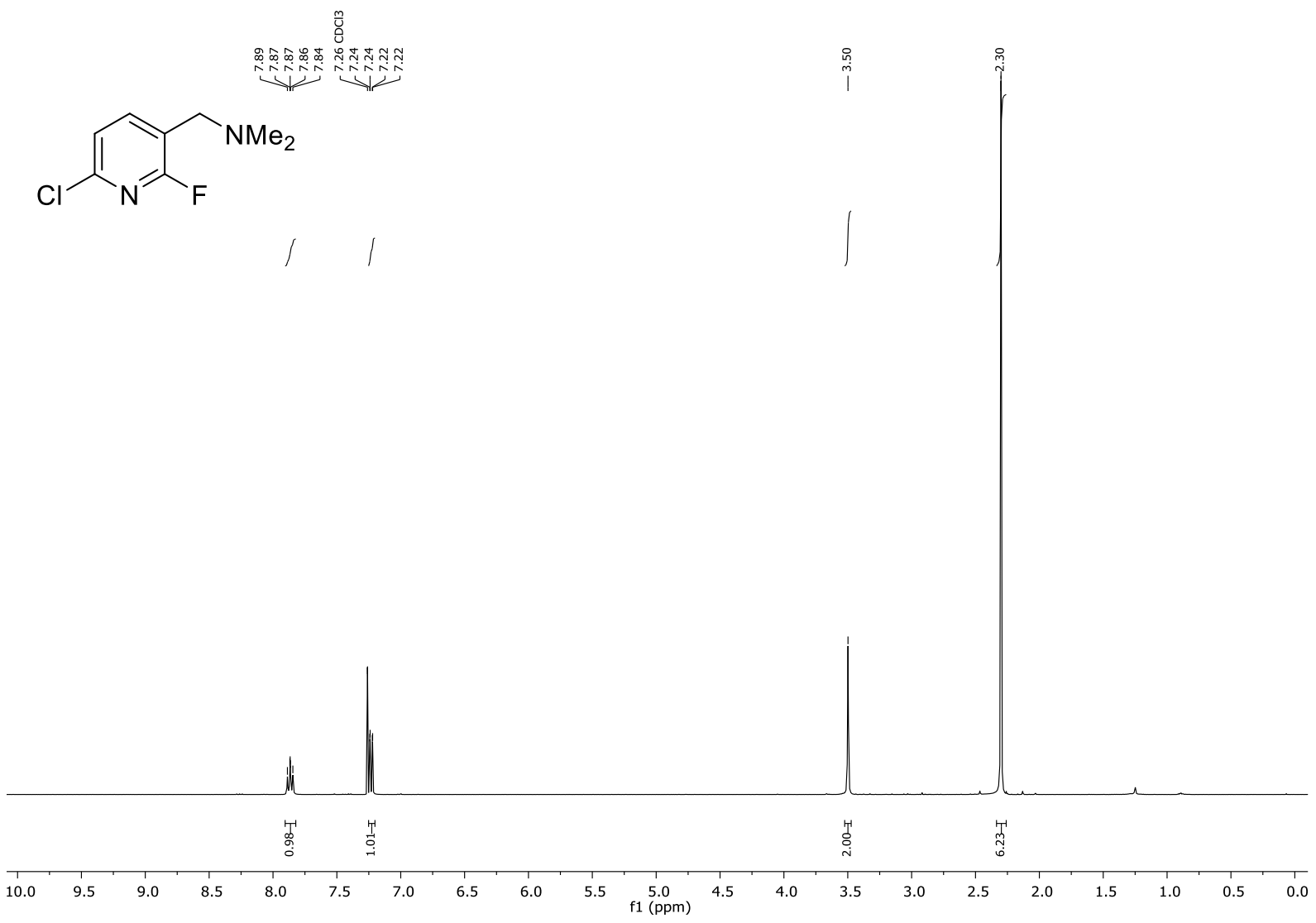

${ }^{13} \mathrm{C}$-NMR (100 MHz, $\left.\mathrm{CDCl}_{3}\right)$ :

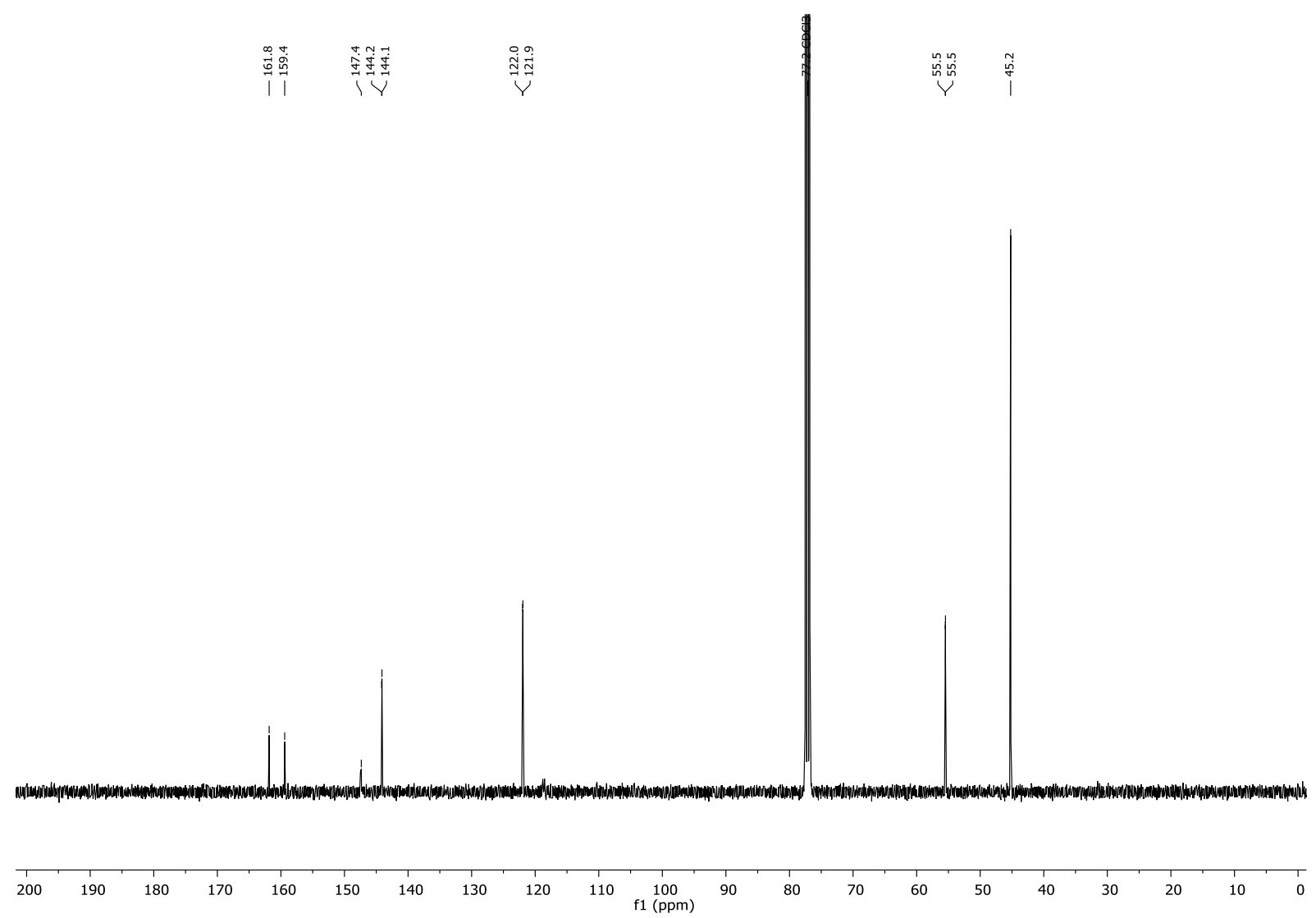




\section{6-Chloro-2-fluoro-3-(chloromethyl)pyridine}

${ }^{1} \mathrm{H}-\mathrm{NMR}$ (400 MHz, $\left.\mathrm{CDCl}_{3}\right)$ :

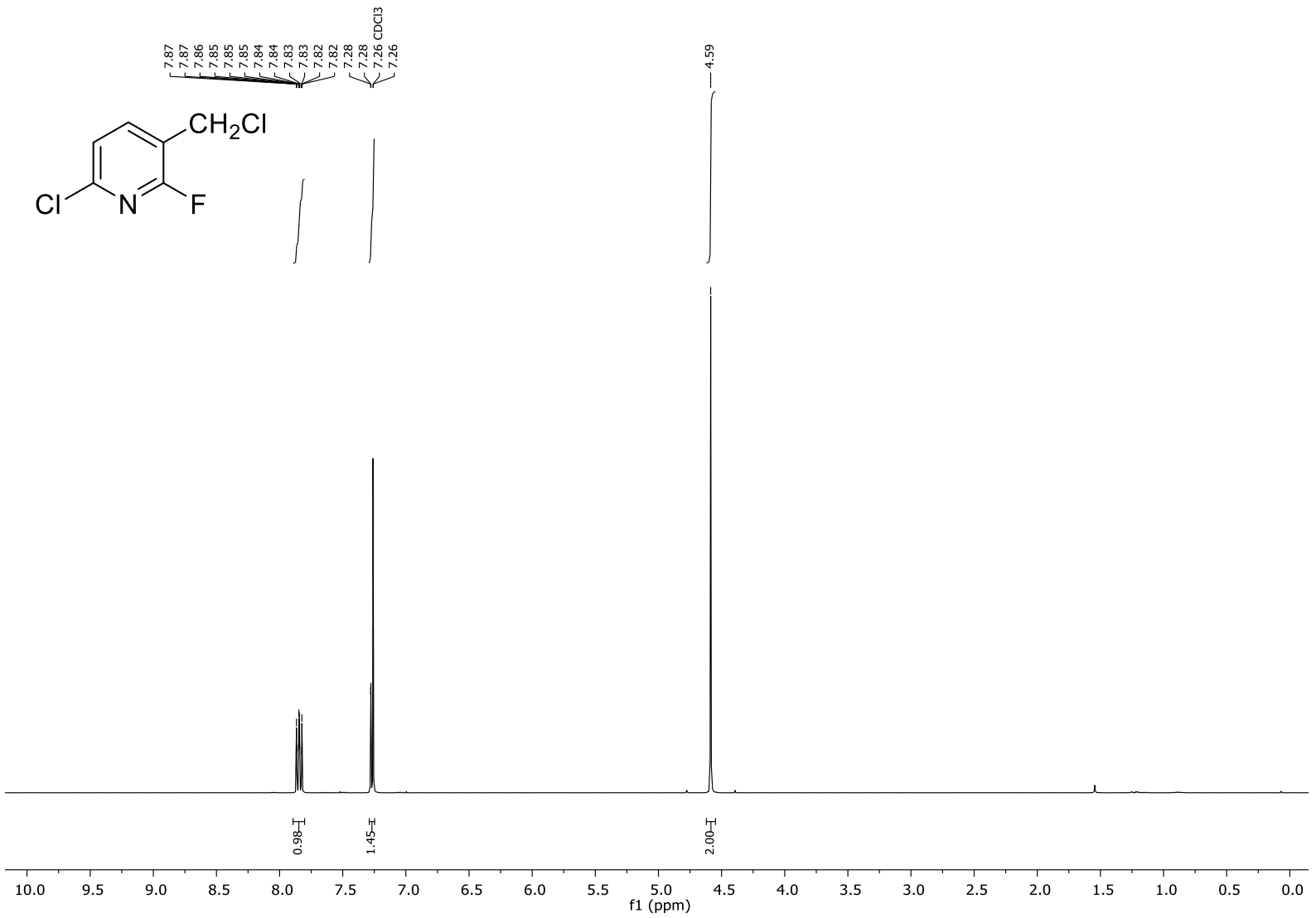

${ }^{13} \mathrm{C}-\mathrm{NMR}\left(100 \mathrm{MHz}, \mathrm{CDCl}_{3}\right)$ :

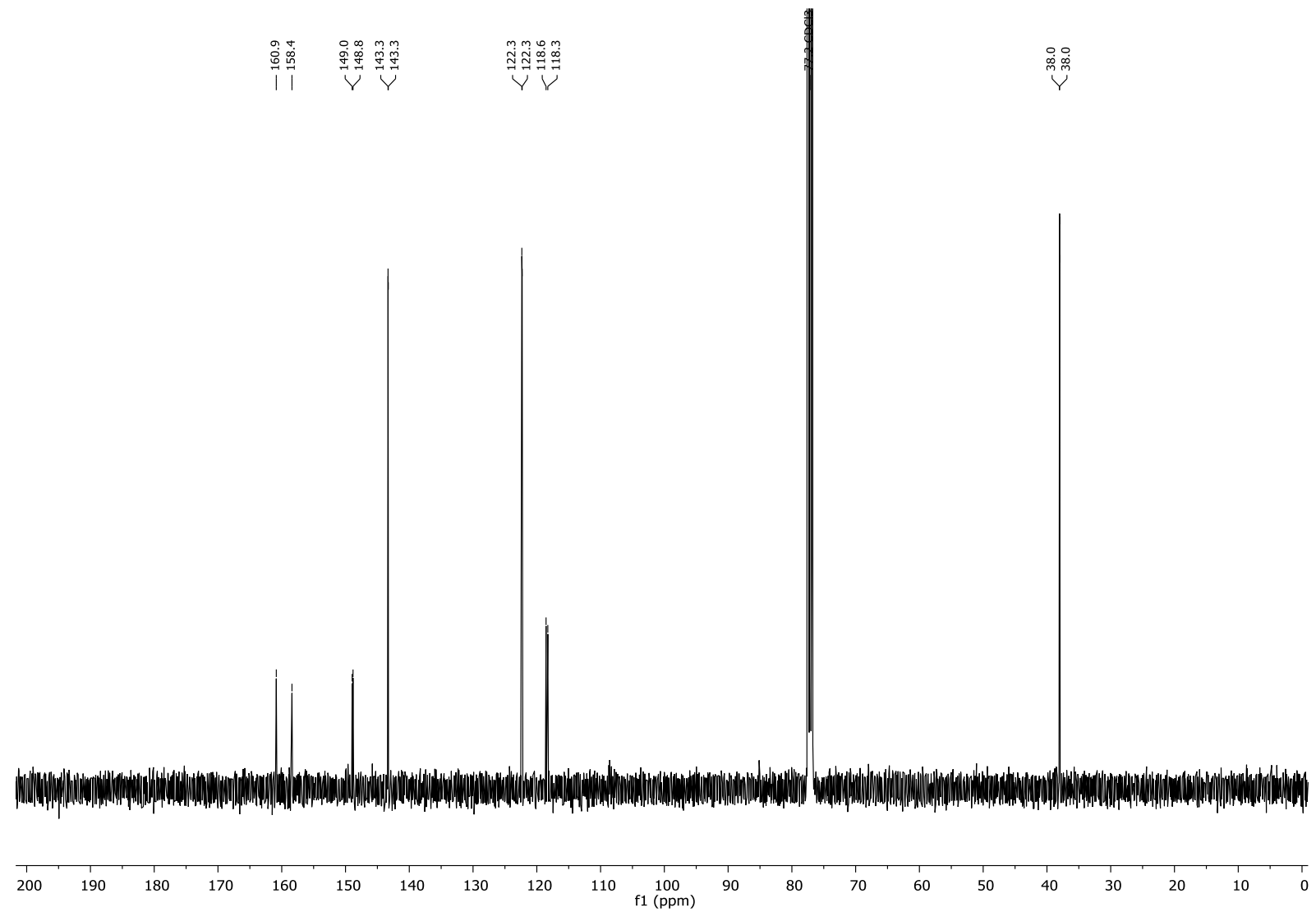


6-Chloro-2-fluoro-3-(iodomethyl)pyridine (z)

${ }^{1} \mathrm{H}-\mathrm{NMR}$ (400 MHz, $\left.\mathrm{CDCl}_{3}\right)$ :

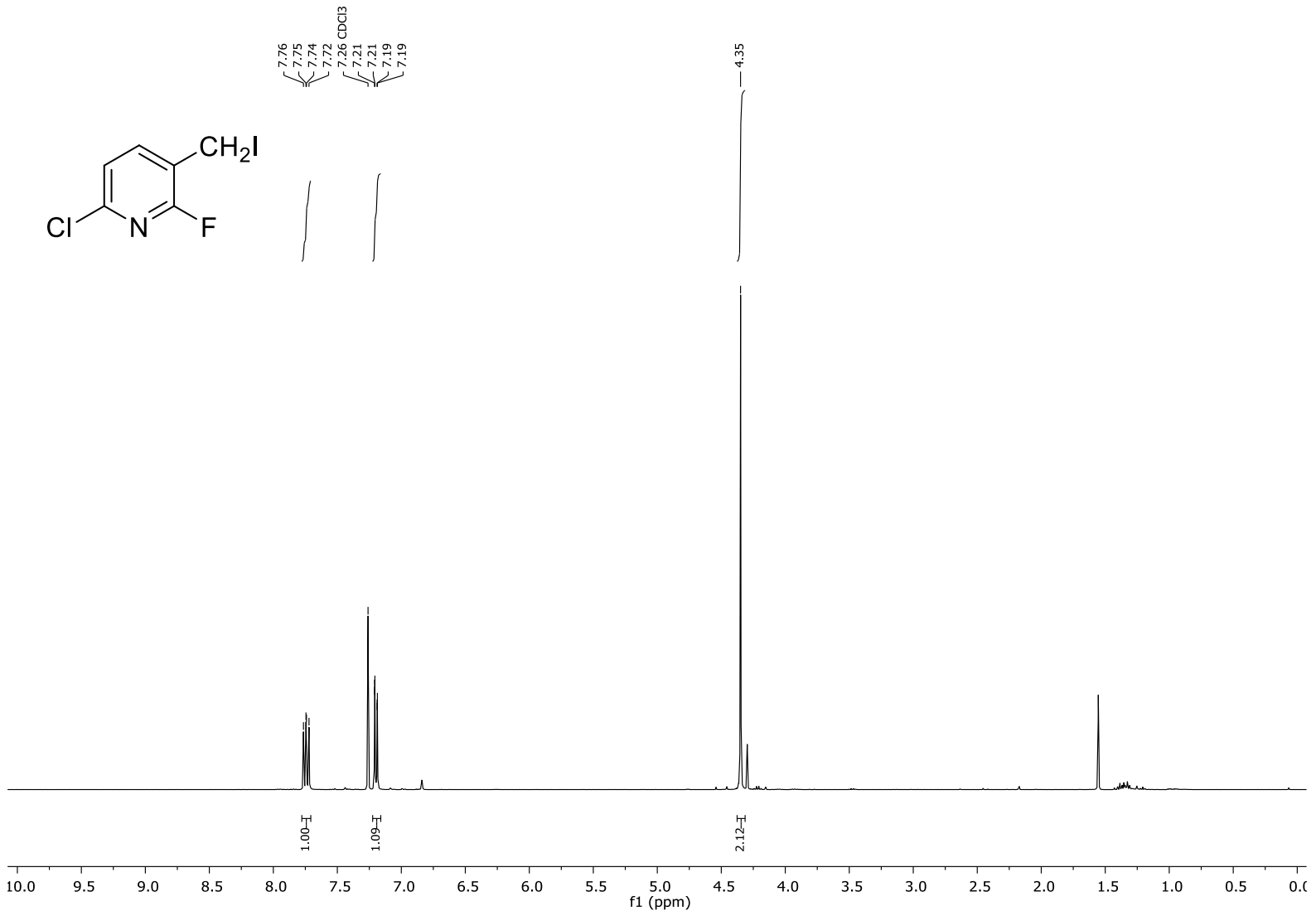

${ }^{13} \mathrm{C}-\mathrm{NMR}$ (100 MHz, $\left.\mathrm{CDCl}_{3}\right)$ :

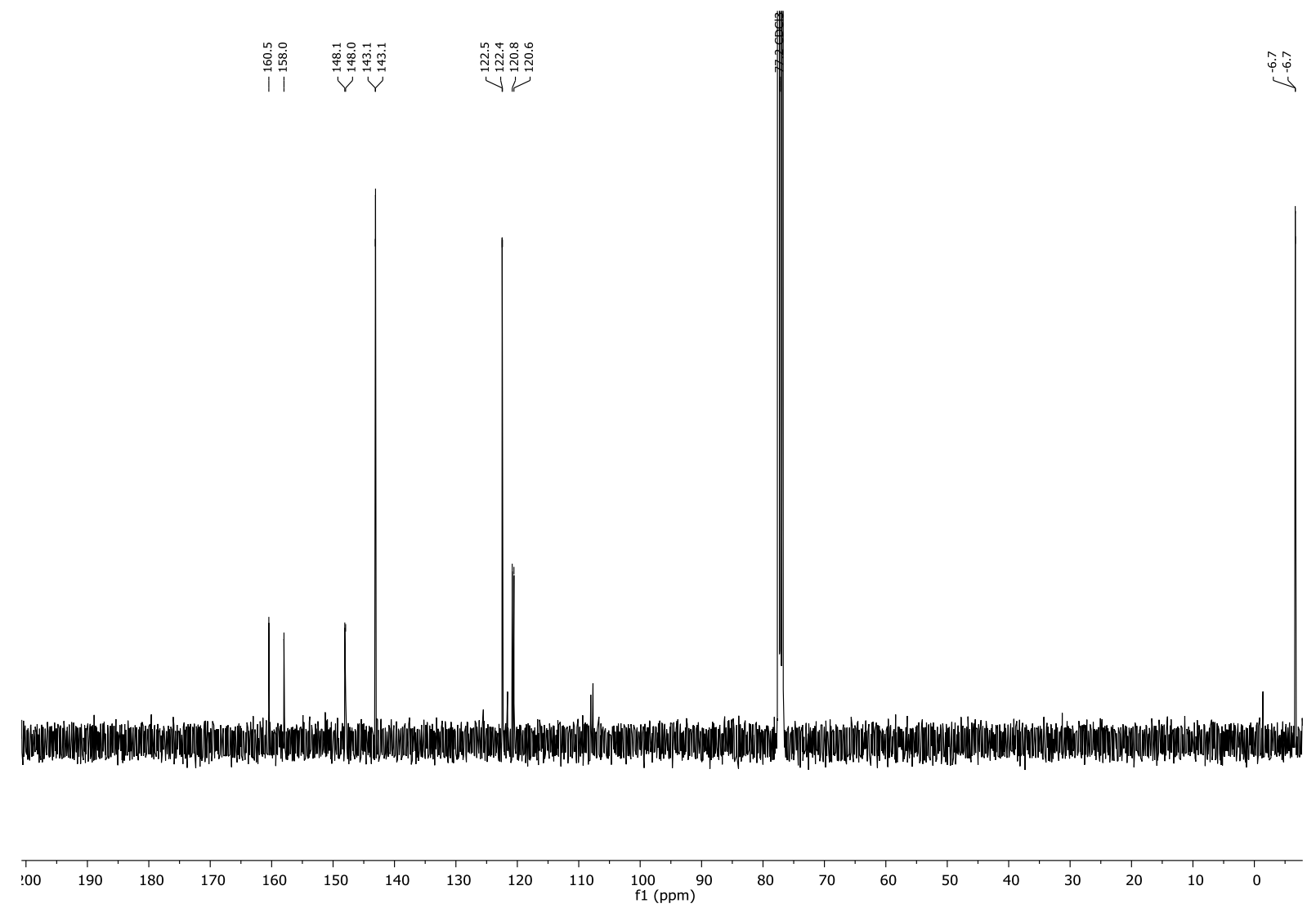


1-(6-Chloro-2-(methylthio)pyridin-3-yl)- $N, N$-dimethylmethanamine

${ }^{1} \mathrm{H}-\mathrm{NMR}$ (400 MHz, $\left.\mathrm{CDCl}_{3}\right)$ :

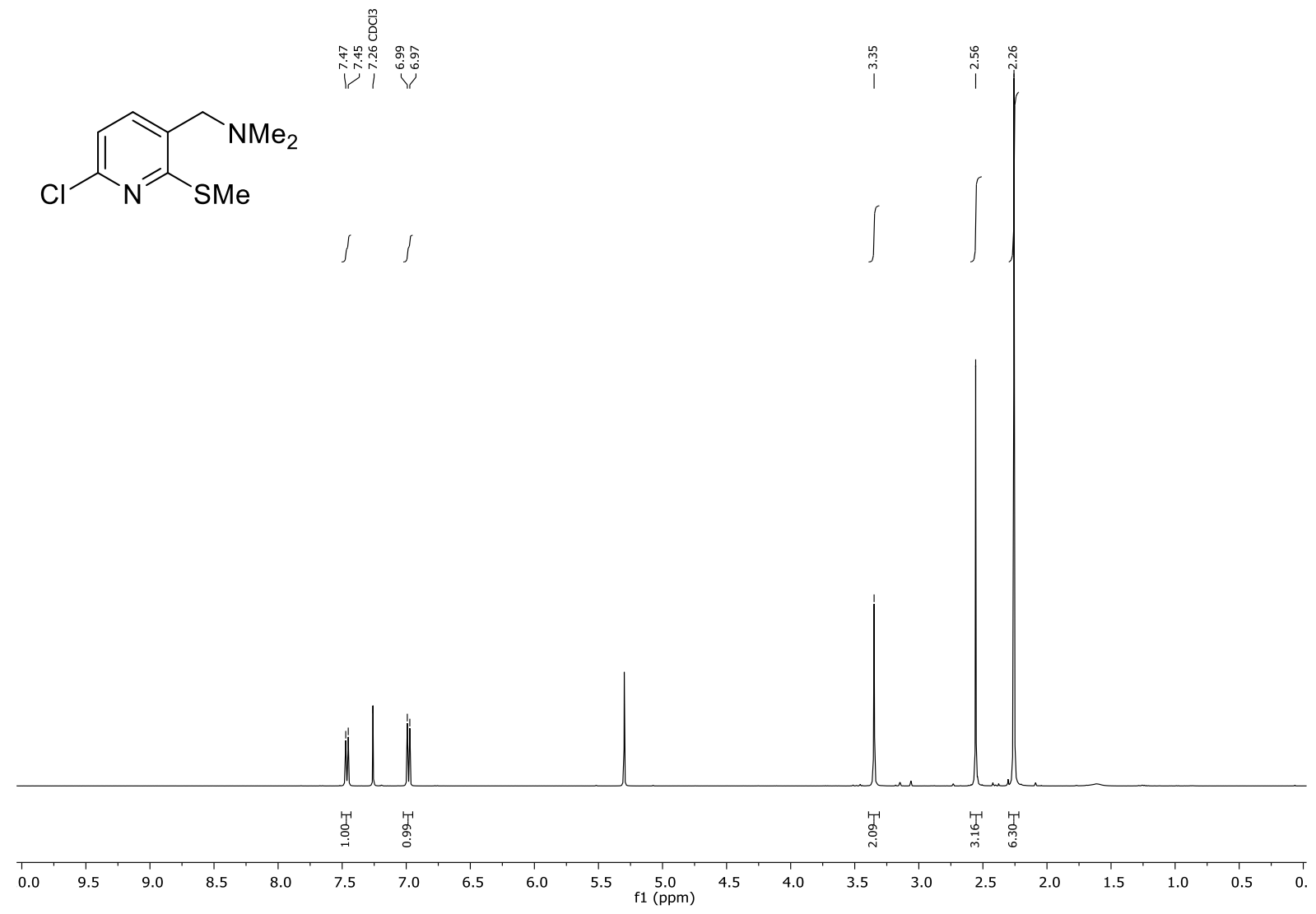

${ }^{13} \mathrm{C}-\mathrm{NMR}\left(100 \mathrm{MHz}, \mathrm{CDCl}_{3}\right)$ :

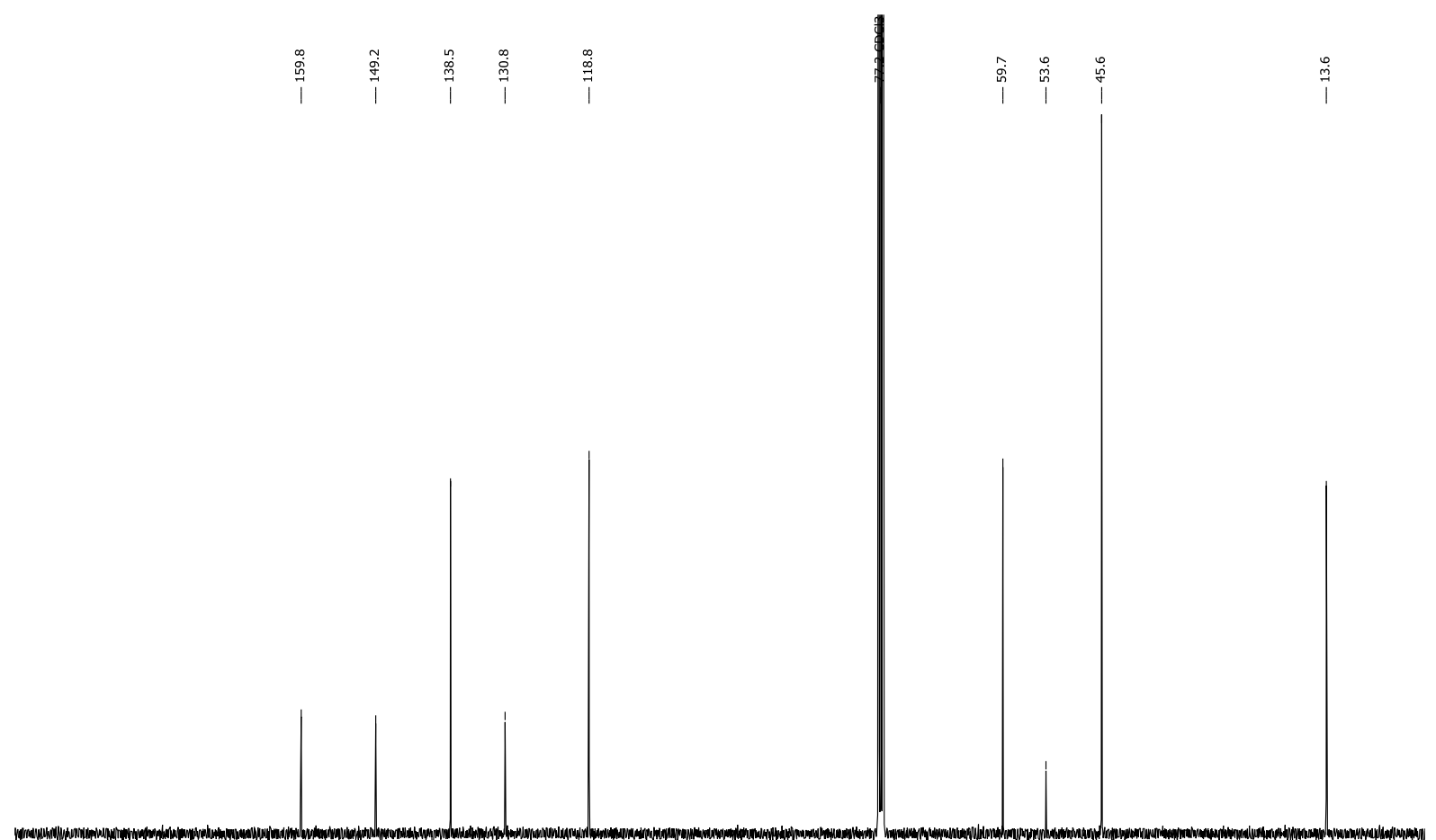

$! 00$

$120 \quad 110 \quad \frac{100}{100}$

80

$60 \quad 50$

$20 \quad 10 \quad c$ 
6-Chloro-3-(chloromethyl)-2-(methylthio)pyridine

${ }^{1} \mathrm{H}-\mathrm{NMR}$ (400 MHz, $\left.\mathrm{CDCl}_{3}\right)$ :

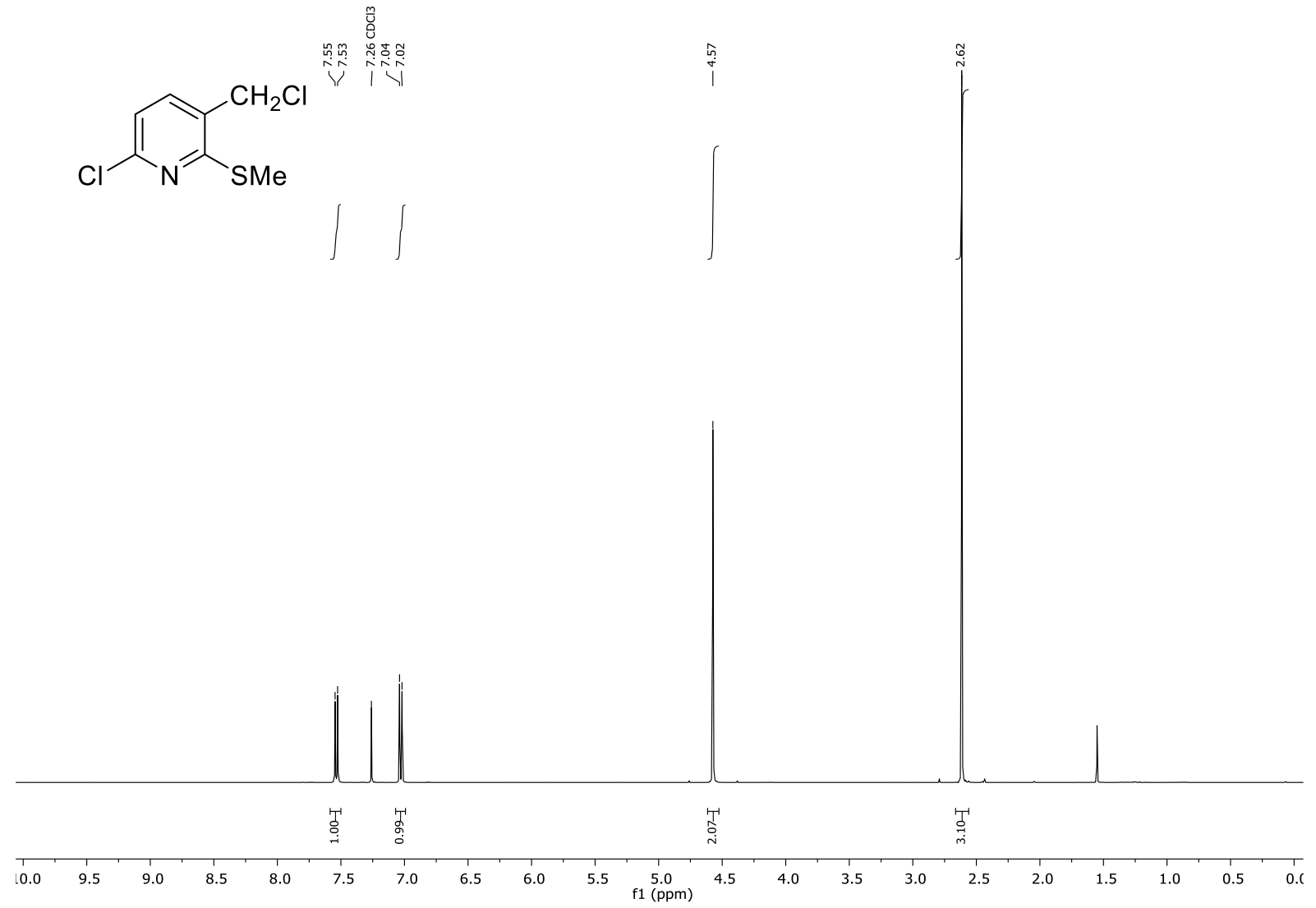

${ }^{13} \mathrm{C}-\mathrm{NMR}$ (100 MHz, $\left.\mathrm{CDCl}_{3}\right)$ :

兽
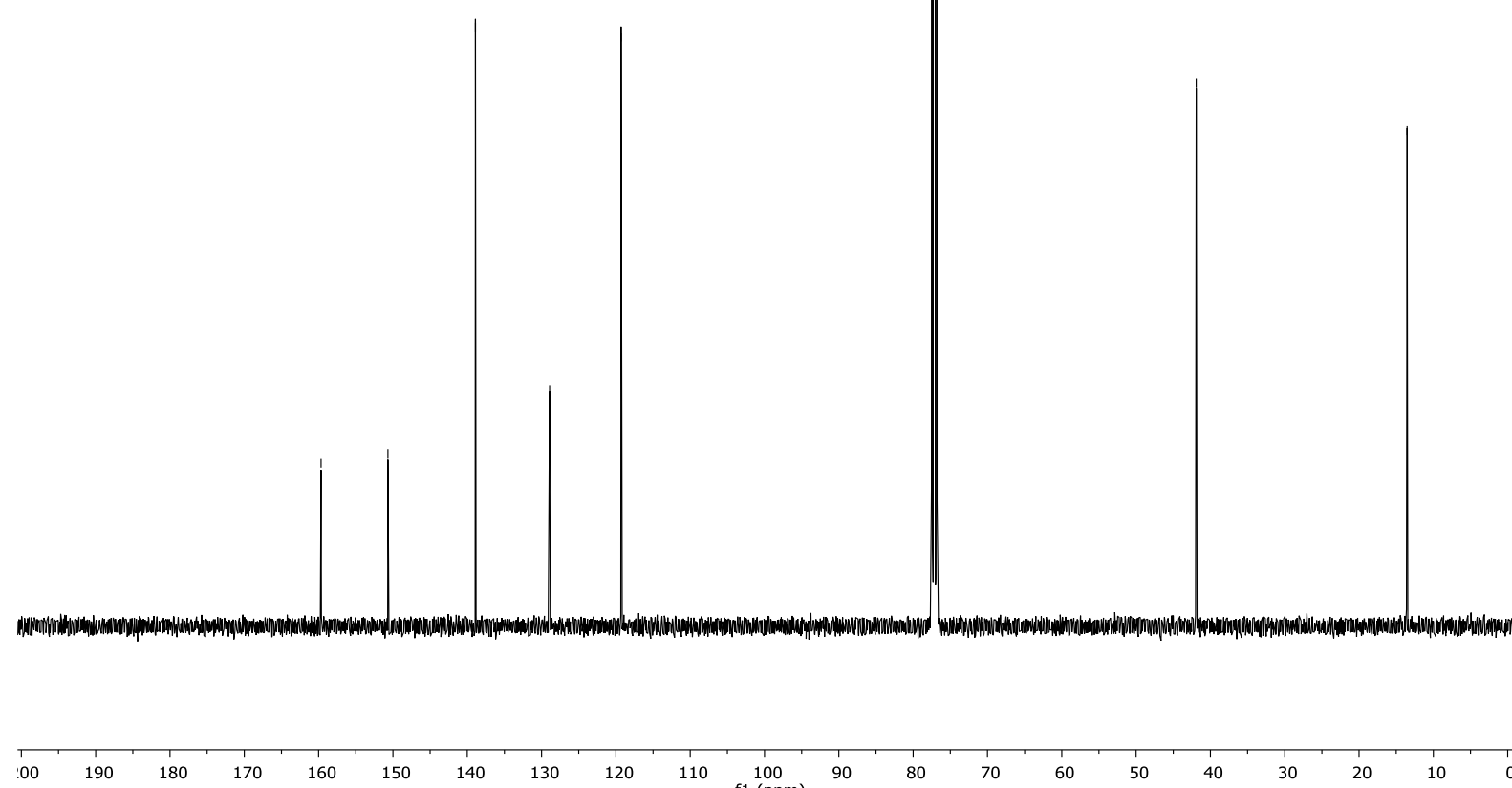

$140 \quad 130$

120

$110 \quad \begin{gathered}100 \\ \mathrm{f} 1(\mathrm{ppm})\end{gathered}$

$90 \quad 80$

(10) 
6-Chloro-3-(iodomethyl)-2-(methylthio)pyridine (11c)

${ }^{1} \mathrm{H}-\mathrm{NMR}$ (400 MHz, $\left.\mathrm{CDCl}_{3}\right)$ :

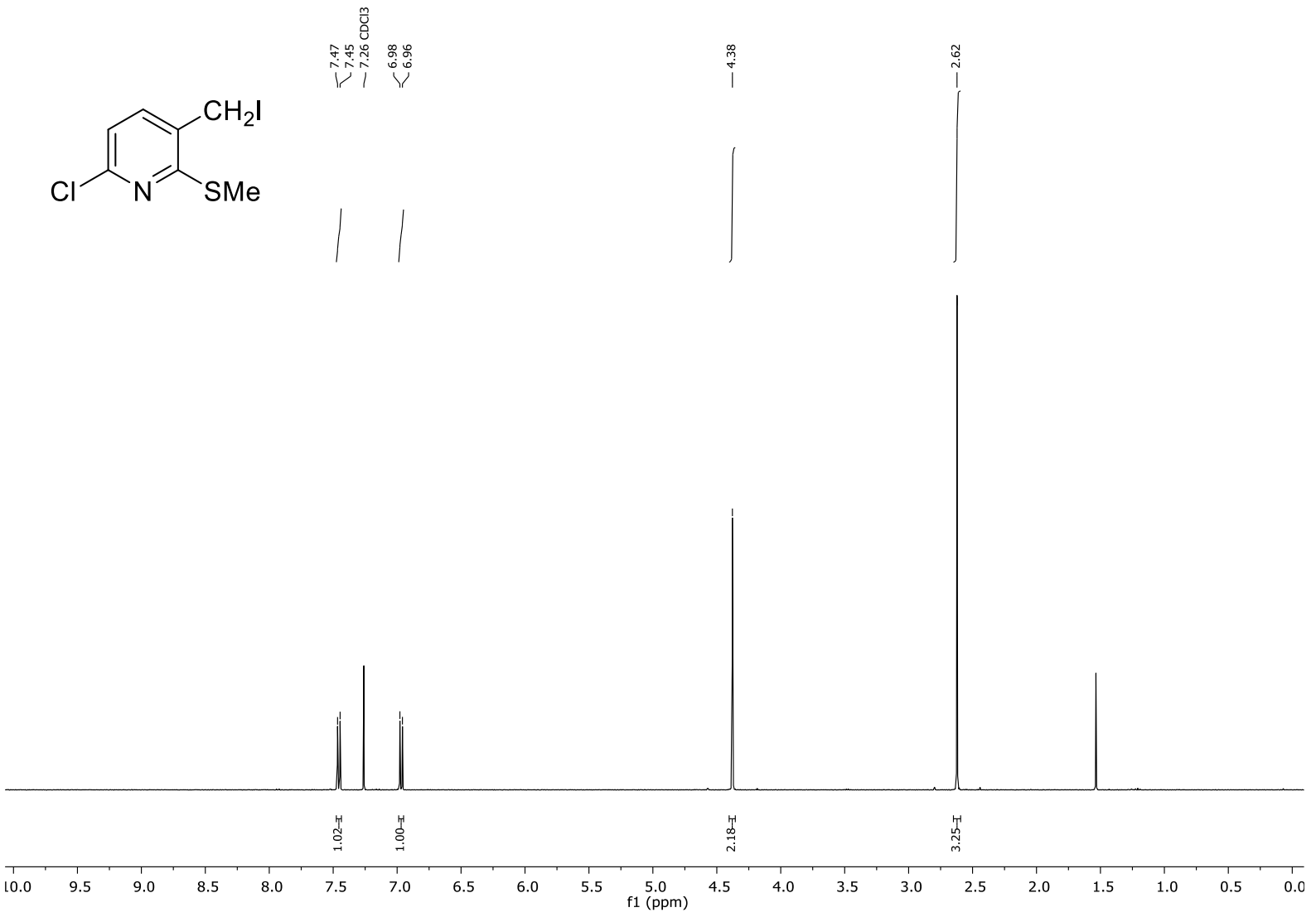

${ }^{13} \mathrm{C}-\mathrm{NMR}$ (100 MHz, $\left.\mathrm{CDCl}_{3}\right)$ :

$$
\text { 总 }
$$

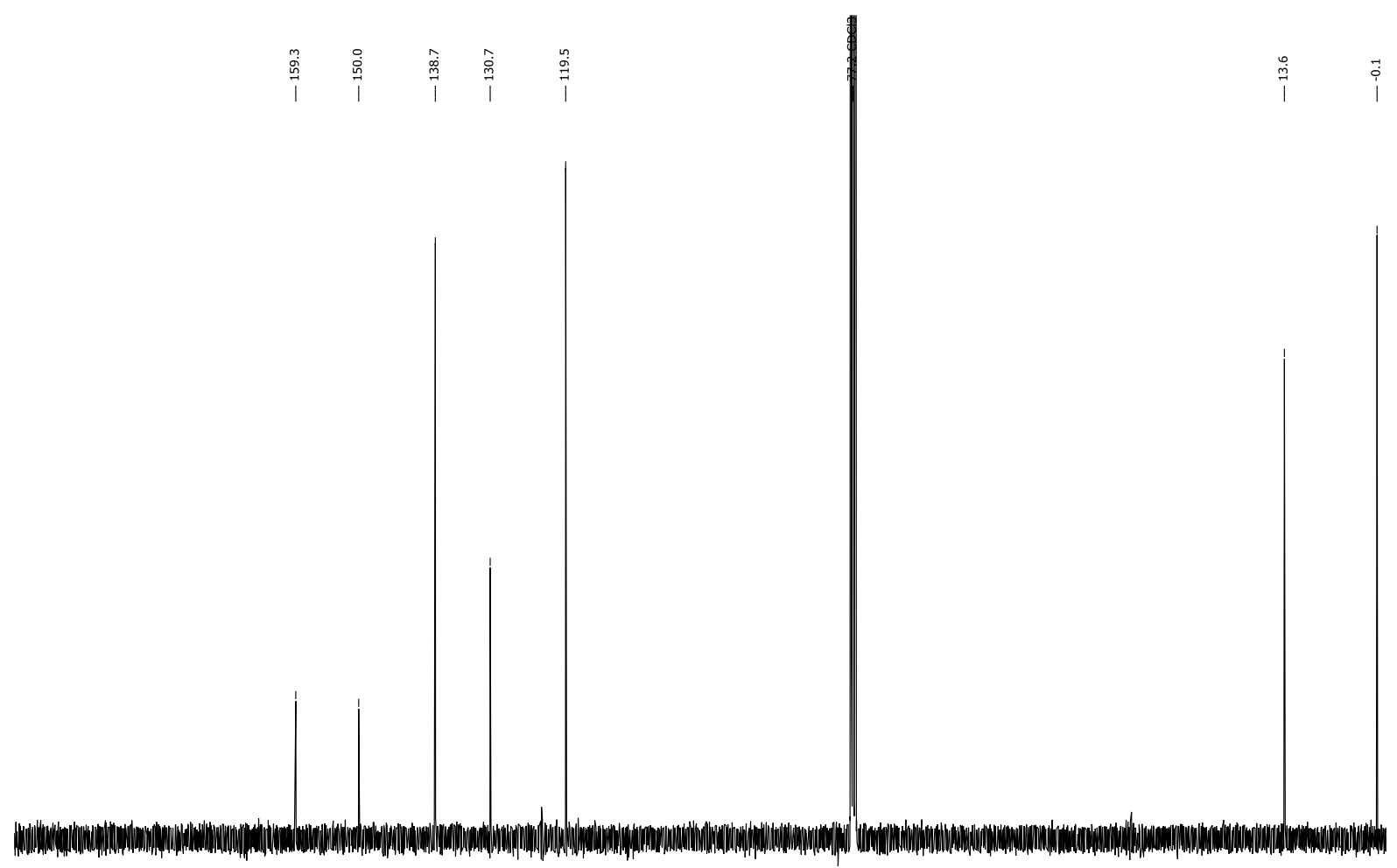

